\title{
Search for High-Mass Top Quark Pair Resonances with the CMS Experiment
}

\author{
By \\ Christine Angela McLean \\ B.S. (The College of William and Mary) 2012 \\ M.S. (University of California, Davis) 2013 \\ DISSERTATION
}

Submitted in partial satisfaction of the requirements for the degree of

DOCTOR OF PHILOSOPHY

in

PHYSICS

in the

OFFICE OF GRADUATE STUDIES

of the

UNIVERSITY OF CALIFORNIA

DAVIS

Approved:

Robin Erbacher (Chair)

Maxwell Chertok

John Conway

Committee in Charge

2018 
Copyright (c) 2018 by Christine Angela McLean All rights reserved. 
In memory of my mom, Mary C. McLean, without whose love, support, and encouragement I would not be where I am today. 


\section{Contents}

1 Introduction $\quad 1$

2 Theory Motivation 3

2.1 The Standard Model . . . . . . . . . . . . . . . . 3

2.1.1 A Brief Introduction to Quantum Field Theory . . . . . . . . . . . 4

2.1.2 The Standard Model Particles . . . . . . . . . . . . . . . 6

2.1.3 The Fundamental Forces . . . . . . . . . . . . . . . . 8

2.2 Beyond the Standard Model . . . . . . . . . . . . . . . . 16

2.2.1 Standard Model Limitations . . . . . . . . . . . . . 16

2.2.2 Heavy $t \bar{t}$ Resonances . . . . . . . . . . . . . . . . . 18

3 Experimental Apparatus $\quad 20$

3.1 The Large Hadron Collider . . . . . . . . . . . . . . . . . . . . . 20

3.1.1 High Luminosity LHC . . . . . . . . . . . . . . 23

3.2 The Compact Muon Solenoid . . . . . . . . . . . . . . 23

3.2.1 CMS Coordinate System ................... 24

3.2.2 CMS Tracker System . . . . . . . . . . . . . . 27

3.2.3 Electromagnetic Calorimeter . . . . . . . . . . . . . 30

3.2.4 Hadronic Calorimeter . . . . . . . . . . . . . . . . . 33

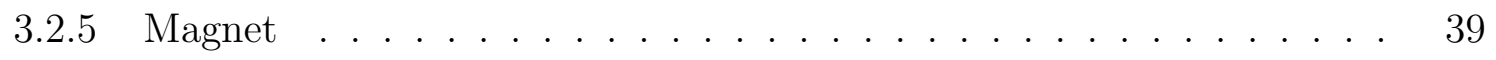

3.2 .6 Muon System . . . . . . . . . . . . . . . . . . 39 
3.2 .7 Trigger and Data Acquisition $\ldots \ldots \ldots \ldots \ldots$

4 Event Reconstruction $\quad 56$

4.1 Particle Flow . . . . . . . . . . . . . . . . . . . 56 5

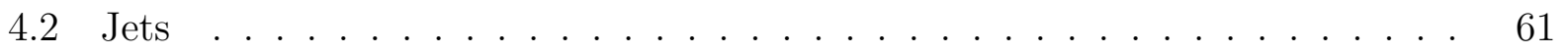

4.2 .1 Jet Clustering . . . . . . . . . . . . . . . . . . . . 61

4.2 .2 Pileup Removal Algorithms . . . . . . . . . . . . . . . . 6 63

5 Top Tagging Studies 65

5.1 Simulated Samples, Object Definitions and Event Selection . . . . . . . . 66

5.2 Top Tagging Methods . . . . . . . . . . . . . . . . . . . . 68

5.2 .1 Groomed Masses . . . . . . . . . . . . . . . . . . 68 68

$5.2 .2 \quad$ N-Subjettiness . . . . . . . . . . . . . . . . . . . . . . . . . . . . . . 69

5.2 .3 b Quark Tagging . . . . . . . . . . . . . . . . . . 70

5.2 .4 CMS Top Tagger $\ldots \ldots \ldots \ldots \ldots$

$5.2 .5 \quad$ HEPTopTagger . . . . . . . . . . . . . . . . 71

5.2 .6 Shower Deconstruction . . . . . . . . . . . . . . . 72

5.3 Distribution of Top Tagging Discriminating Variables . . . . . . . . . . 73

5.3 .1 Mass and N-subjettiness . . . . . . . . . . . . 73

5.3 .2 Combined Tagger Variables . . . . . . . . . . . . . 755

5.4 Tagger Combinations . . . . . . . . . . . . . . . . . . . 76

5.5 Tagger Efficiency Kinematic Dependence . . . . . . . . . . . . . . 78

6 Analysis Selections 83

6.1 Samples . . . . . . . . . . . . . . . . . . 85

$6.1 .1 \quad$ Data Samples . . . . . . . . . . . . . . . . . . . 85

6.1.2 Simulated Background Samples _. . . . . . . . . . . . 85

6.1 .3 Simulated Signal Samples _... . . . . . . . . . . 8 85

6.1 .4 Pileup Reweighting . . . . . . . . . . . . . . . . 87 
6.2 Event Selection . . . . . . . . . . . . . . . . . . . . . 88

6.2 .1 Trigger Selection . . . . . . . . . . . . . . . . 88

6.2.2 Vertex Selection and Pileup Mitigation . . . . . . . . . 91

6.2 .3 Jet Reconstruction . . . . . . . . . . . . . . . . . . . . . . . 92

6.2 .4 Top $p_{T}$ reweighting $\ldots \ldots \ldots \ldots \ldots \ldots$

6.2 .5 Event Preselection . . . . . . . . . . . . . . . . . . 94

6.2.6 Top Tagging Algorithms _... . . . . . . . . . . . . 96

6.2 .7 Subjet b-tagging . . . . . . . . . . . . . . . 97

6.2.8 Signal Region and Event Categorization $\ldots \ldots \ldots$

7 Background Estimation and Event Kinematics 104

7.1 Data-derived Backgrounds . . . . . . . . . . . . . . . . . 104

7.2 Closure Tests for the CMS Top Tagger V2H Analysis . . . . . . . . . . 108

7.3 Event Kinematics . . . . . . . . . . . . . . . . . . . . . . . 110

8 Systematic Uncertainties 114

8.1 Top Tagging and Subjet b-Tagging Scale Factor Uncertainties . . . . . . 115

8.2 Jet Energy Scale . . . . . . . . . . . . . . . . . . . . . 115

8.3 Jet Energy Resolution . . . . . . . . . . . . . . . . . . . . . . 116

8.4 Parton Distribution Function Uncertainties . . . . . . . . . . . . . . . 116

8.5 Pileup Uncertainties . . . . . . . . . . . . . . . . . . . . 117

8.6 Top $p_{T}$ Reweighting Uncertainties . . . . . . . . . . . . . . . . . 117

8.7 SM $t \bar{t}$ Uncertainties . . . . . . . . . . . . . . . . . . . . . . . . . . 117

8.8 Multijet Background Uncertainties _. . . . . . . . . . . . . . 117

9 Statistical Interpretation and Results 119

10 Conclusions 
A Muon Hardware Projects $\quad[128$

A.1 CSC Hardware . . . . . . . . . . . . . . . . . . . . . . . . 128

A.2 GEM Hardware . . . . . . . . . . . . . . . . . . . . . 129

A.2.1 VFAT2 Testing . . . . . . . . . . . . . . . . 129

A.2.2 EYETS . . . . . . . . . . . . . . . . . 133

B Additional Kinematic Plots

C $\operatorname{Top} p_{T}$ Reweighting $\quad 148$

References 
Christine Angela McLean

February 2018

Physics

Search for High-Mass Top Quark Pair Resonances with the CMS Experiment

\section{$\underline{\text { Abstract }}$}

I present a search for new massive particles decaying to a pair of top quarks with the CMS detector at the LHC, using $36 \mathrm{fb}^{-1}$ of proton-proton collision data recorded in 2016, at a center-of-mass energy of $13 \mathrm{TeV}$. In the high mass ranges accessible by the LHC at these energies, the top quarks are produced with high transverse momenta: the products of hadronically decaying top quarks are reconstructed as a single jet. Specific reconstruction algorithms and selections are employed to address the identification of boosted top quark signatures. The search is performed by measuring the invariant mass distribution of the top-quark pair and testing for deviations from the expected Standard Model background. The results are presented in terms of expected upper limits on the production cross section of several models, including those of Randall-Sundrum Kaluza-Klein gluon and $Z^{\prime}$ boson production. The results are then compared with those of previous searches. 


\section{Acknowledgments and Thanks}

I would like to thank my advisor, Robin Erbacher, for being a wonderful mentor and friend. Thank you for guiding me through my $\mathrm{PhD}$ and pushing me to make the most out of every opportunity. You not only cared about the quality of my work, but also made sure that I enjoyed my $\mathrm{PhD}$ experience. For this, I am especially grateful. And thanks for always making time for CMS week fondue dinners!

Thanks to Justin Pilot, for acting as a kind of second advisor. Thanks for answering all of my coding and analysis questions and for making sure I was never stuck for too long. Thank you for picking me up from the airport when I first arrived at CERN and showing

me the ropes. Thanks for the countless rides around SwitzerFrance. And thank you for your friendship.

I am grateful for the support of the entire Davis CMS group. I would like to give special thanks to the other members of my dissertation committee, Max Chertok and John Conway. Thanks to Francesca, Rachel, and Reyer for being good friends and colleagues at CERN. Thank you, too, to the UC Davis Physics department for supporting me throughout my years as a graduate student.

I enjoyed being a member of the CMS experiment and working with so many great people. Thanks to the rest of the $Z^{\prime}$ all-hadronic team, Jim, Maral, and Sal. Thanks to Gregor, who led our top tagging studies, and to Jared who supervised my GEM hardware activities. I learned a lot from you and from my many other CMS colleagues.

To my UC Davis cohort, thank you for being an amazing group of friends. You made the difficulties of grad school classes not only bearable, but fun. Thanks to Josh and Kathryn, who befriended me quickly at the beginning of our first year and accompanied me on many adventures. To Brian and Austin, who visited me in Europe when I was missing my California friends. And to Christina, who spent long hours working with me as we both prepared to finish our graduate careers and move on to postdoc positions. 
I would like to thank all of the other friends I made during the course of my graduate career. Thanks to all of the people I befriended in Davis, through physics and trivia. I'm grateful to have been able to make such great friends at CERN, Fermilab, and various physics schools. Every time I moved, each set of new friends made all of the sad goodbyes worthwhile. I would especially like to thank two of my friends at CERN, Paul and Juliana, who looked out for me after my mom died.

I am grateful for all of the mentors I had before entering graduate school. I would especially like to thank my undergraduate advisor, Todd Averett. Thank you for supporting me from the summer after my freshman year, when I had very little physics background, through the summer after my senior year, after I had completed my honors thesis. I was able to explore so many different aspects of experimental physics research and get a feel for what I was really passionate about. Most importantly, you made research fun.

I would like to thank the many members of my extended family who have supported me throughout my life. Special thanks to my aunt, Trisha, who gave me a home away from home in California and who would be there at a moment's notice whenever I needed anything, especially help with packing. To my close high school and college friends, thank you for your friendship and encouragement.

And finally, thank you to my immediate family for your love. Thanks to my mom, Mary, for always encouraging my passion for math and science, from teaching me to divide with barbies when I was four to pushing me to do research as an undergraduate. Thanks to my dad, Jeff, for sharing your sense of curiosity and humor and for always being there when I was stressed. And to my little brother, Jeffrey, thanks for sharing your love and motivating me to be a better person. I have drawn so much inspiration from all of you. 


\section{Chapter 1}

\section{Introduction}

This dissertation describes the search for heavy new particles that decay exclusively to pairs of top quarks. The analysis uses $36 \mathrm{fb}^{-1}$ of data collected in 2016 by the Compact Muon Solenoid (CMS) experiment. The data were produced by colliding protons at a center-of-mass energy of $13 \mathrm{TeV}$, using the Large Hadron Collider (LHC) at the European Organization for Nuclear Research (CERN), located near Geneva, Switzerland. Specifically, the analysis looks for a top-antitop-quark pair in which each top quark decays hadronically and is reconstructed as a single "top jet". Novel top tagging techniques, used to identify top jets, were optimized for this analysis.

Chapter 2 introduces the theoretical motivation for high energy particle physics experiments. An overview of the Standard Model of particle physics is given. Important limitations of the Standard Model are introduced, and a theoretical model that proposes solutions to a few of these problems is presented. This model motivates the physics search that is the subject of this dissertation. In Chapter 3, the experimental apparatus is described. This

includes both the LHC and planned upgrades, as well as all of the subdetectors that comprise CMS.

Event reconstruction techniques, including jet clustering and pileup removal methods, are presented in Chapter 4. The optimization and performance of the CMS Run II top tagger 
are described in Chapter 5 .

Chapter 6 presents the event selection for the $Z^{\prime} \rightarrow t \bar{t}$ search. Background estimation methods and resulting kinematic distributions are examined in Chapter 7. The systematic uncertainties considered in the analysis are discussed in Chapter 8. Then, the results are presented in Chapter 9, while Chapter 10 summarizes the analysis.

Appendix A describes the main CMS muon hardware projects in which I was involved. My roles in testing Cathode Strip Chamber (CSC) hardware and as a CSC detector-oncall shifter are presented. Additionally, my work in preparing for and assisting with the installation of the Gas Electron Multiplier demonstrator is discussed.

Appendices $\mathrm{B}$ and $\mathrm{C}$ provide extra kinematic and top $p_{T}$ reweighting plots for the $Z^{\prime}$ analysis. 


\section{Chapter 2}

\section{Theory Motivation}

This chapter provides an introduction to the Standard Model of particle physics, which describes the elementary particles and their interactions. Limitations of the Standard Model are discussed. The Randall-Sundrum model of extra dimensions, which attempts to address some of these limitations, is introduced.

\subsection{The Standard Model}

The Standard Model (SM) is a theory of particle physics that uses the formalisms of quantum field theory (QFT) to describe the elementary particles and the forces through which they interact. Of the four fundamental forces, the SM successfully describes three: the electromagnetic, strong, and weak interactions. In 1961, building on the work of many other scientists, Glashow made the first step toward the Standard Model when he proposed a unified model of the electromagnetic and weak interactions [1]. In 1967, the Higgs mechanism was incorporated into this electroweak model by Salam and Weinberg [2]. Then in 1973, the current mathematical description of the strong force, quantum chromodynamics (QCD), was finalized [3, 4, 5]. Despite the fact that some physical phenomena remain unexplained, the SM is the most comprehensive theory of particle physics to date and has been highly successful in accurately predicting experimental results [6]. 


\subsubsection{A Brief Introduction to Quantum Field Theory}

Before the emergence of quantum mechanics, classical physics was used to describe the universe at the macroscopic scale. In the early 1900s, it was found that the classical formulation of physics was inadequate for describing processes at the subatomic level. Breakthroughs such as Planck's formula for blackbody radiation, Einstein's explanation of the photoelectric effect, and the discovery of the Compton effect indicated that electromagnetic radiation, classically understood to exhibit wave-like properties, was made up of particles called photons [7. Additionally, the observation of electron diffraction proved that electrons also exhibited wave-like properties. This wave-particle duality of matter broke with the classical notion of the fundamental difference between waves and particles. Quantum mechanics was therefore born out of a necessity for a mathematical description of subatomic particles.

The observation of the self-interference of individual photons and electrons led to the wave function formalism of quantum mechanics [7]. In this theoretical framework, each particle is described by a linear equation, called a wave function $(\psi)$, which obeys the superposition principle. The wave function allows for the measurement of physical properties of the particle, such as position and momentum. Free particles are described by plane-wave equations and have continuous energy spectra. Bound particles, on the other hand, have discrete energy levels arising from the standing-wave-like equations that describe them.

During the time period in which quantum mechanics was being formulated, relativity theory was also developed. Therefore, efforts were made to develop relativistic wave equations. An early attempt produced the Klein-Gordon equation:

$$
\left[\frac{\partial^{2}}{\partial t^{2}}-\nabla^{2}+m^{2}\right] \psi(\mathbf{x}, t)=0,
$$

resembling a classical wave equation with an additional mass squared term [8]. This equation was successful in that it was invariant under Lorentz transformations, necessary for relativistic mechanics, and that it produced energy solutions consistent with those expected 
for a free relativistic particle with mass $m$. However, it also gave rise to negative energy solutions and associated negative probability densities. At the time, this was thought to be unphysical. In addition to this apparent failing, the Klein-Gordon equation also failed to provide particles with a spin degree of freedom and did not properly describe the hydrogen atom [8]. However, it was later found that spin-zero particles were successfully described by the Klein-Gordon equation.

Problems with the Klein-Gordon equation were attributed to the fact that it is a secondorder differential equation in time. Therefore, Dirac looked to create a wave equation that was linear in space and time derivatives:

$$
\left(i \gamma^{\mu} \partial_{\mu}-m\right) \psi(\mathbf{x}, t)=0
$$

where $\partial_{\mu}$ indicates the spacetime derivatives 8 . This equation successfully produced the energy solutions expected for a free particle and was more attractive than the Klein Gordon equation, as it only allowed for positive probability densities. It also provided an additional spin degree of freedom, indicating the existence of spin- $\frac{1}{2}$ particles. The solutions to the Dirac equation led to the interpretation of negative energies as "antiparticles". All particles have an antiparticle, which has the same mass, spin (a type of intrinsic angular momentum), and lifetime, but opposite electric charge. This interpretation of the Dirac equation led to the discovery of the positron (anti-electron), which in turn affirmed the validity of quantum field theory as a mathematical description of particle physics [8].

In reality, the solutions to the Klein-Gordon and Dirac equations are not the standard quantum mechanical single-particle wavefunctions, but instead describe fields. The equations of motion and interactions of fields can be derived from the Lagrangian density $\mathcal{L}$, which is a function of one or more fields and their derivatives [9]. A continuous transformation of a field that leaves $\mathcal{L}$ and the equations of motion invariant is called a symmetry. These gauge transformations form corresponding gauge groups, which have associated group generators. 


\begin{tabular}{c|ccccccc} 
& Particle & Symbol & $\begin{array}{c}\text { Anti- } \\
\text { particle }\end{array}$ & Generation & Spin & $\begin{array}{c}\text { Electric } \\
\text { Charge } \\
{[\mathrm{e}]}\end{array}$ & Mass [MeV] \\
\hline \multirow{5}{*}{ Quarks } & Up & $u$ & $\bar{u}$ & I & $1 / 2$ & $2 / 3$ & 2.2 \\
& Down & $d$ & $\bar{d}$ & I & $1 / 2$ & $-1 / 3$ & 4.7 \\
& Strange & $s$ & $\bar{s}$ & II & $1 / 2$ & $-1 / 3$ & 96 \\
& Charm & $c$ & $\bar{c}$ & II & $1 / 2$ & $2 / 3$ & 1270 \\
& Bottom & $b$ & $\bar{b}$ & III & $1 / 2$ & $-1 / 3$ & 4180 \\
& Top & $t$ & $\bar{t}$ & III & $1 / 2$ & $2 / 3$ & 173210 \\
\hline \multirow{5}{*}{ Leptons } & Electron & $e^{-}$ & $e^{+}$ & I & $1 / 2$ & -1 & 0.511 \\
& Muon & $\mu^{-}$ & $\mu^{+}$ & II & $1 / 2$ & -1 & 105.7 \\
& Tau & $\tau^{-}$ & $\tau^{+}$ & III & $1 / 2$ & -1 & 1776.9 \\
& Neutrino & $\nu_{e}$ & $\bar{\nu}_{e}$ & I & $1 / 2$ & 0 & $<2 \times 10^{-6}$ \\
& $\mu$ Neutrino & $\nu_{\mu}$ & $\bar{\nu}_{\mu}$ & III & $1 / 2$ & 0 & $<2 \times 10^{-6}$ \\
& $\tau$ Neutrino & $\nu_{\tau}$ & $\bar{\nu}_{\tau}$ & III & $1 / 2$ & 0 & $<2 \times 10^{-6}$ \\
\hline \multirow{5}{*}{ Bosons } & Photon & $\gamma$ & $\gamma$ & - & 1 & 0 & 0 \\
& Gluon & $g$ & $\bar{g}$ & - & 1 & 0 & 0 \\
& W & $W^{ \pm}$ & $W^{\mp}$ & - & 1 & \pm 1 & 80385 \\
& Z & $Z$ & $Z$ & - & 1 & 0 & 91187.6 \\
& Higgs & $\mathrm{H}$ & $\mathrm{H}$ & - & 0 & 0 & 125090
\end{tabular}

Table 2.1: Properties of the elementary particles of the Standard Model [10].

Each generator gives rise to a corresponding gauge field. The inclusion of these gauge fields in $\mathcal{L}$ ensures its gauge invariance, i.e. its invariance under the local group transformations. In the quantization of this theory, gauge bosons arise as the quanta of the gauge fields. The gauge bosons are the mediators of the fundamental interactions of the Standard Model. In addition to the gauge bosons, the quantum field theories of the SM (describing all forces except gravity) predict the existence of many fundamental particles. These particles and their properties are described in the next section.

\subsubsection{The Standard Model Particles}

The Standard Model categorizes the elementary particles into different groups, based on their properties, some of which are shown in Table 2.1. In the Standard Model, the matter particles, or fermions, interact via the exchange of the force carriers, or bosons. The spin of 
a particle determines whether it is a fermion, with half-integer spin, or a boson, with integer spin [6]. Bosons follow Bose statistics, and the wave function $(\psi)$ of a pair of identical bosons is symmetric under their exchange. Fermions, however, obey the Dirac equation (Equation 2.1.2 and follow Fermi-Dirac statistics - fermionic wave functions are antisymmetric under the exchange of a pair of identical fermions. Fermions, therefore, obey the Pauli exclusion principle, which states that two identical fermions cannot occupy the same quantum state.

Fermions are further categorized into quarks and leptons. The quarks are spin-1/2 particles, which are separated into three pairs, or generations, of particles. Additionally, each generation comprises one "up-type" quark, with electric charge $+2 / 3$, and one "down-type" quark, with electric charge $-1 / 3$ [6]. (All electric charges described in this dissertation are written in units of the elementary charge $e=1.602 \times 10^{-19} \mathrm{C}$.) The names of the up-type (down-type) quarks are: up, charm, and top (down, strange, and bottom), written in order of increasing mass within each category. Quarks interact via the electromagnetic, weak, strong, and gravitational interactions.

Similar to the quarks, the spin-1/2 leptons also fall into three generations of particle pairs. The first generation consists of the electron, which has charge -1 , and the neutral electron neutrino. The next two generations consist of increasingly heavy charged leptons and their corresponding neutrinos [6]. Of the charged leptons, only the electron is stable. This is due to the fact that it is the lightest charged particle, and its decay would violate total electric charge, a quantity conserved in all particle interactions. All leptons interact via the weak and gravitational forces, and the charged leptons also interact electromagnetically. Unlike the quarks, leptons do not have color charge, and therefore do not interact via the strong force.

The force carriers of the Standard Model are spin-1 particles known as gauge bosons. The particles that interact through each of the forces are characterized by a corresponding type of charge. The massless photon $(\gamma)$ is the mediator of the electromagnetic force, which acts on electrically charged particles. Photons are electrically neutral and therefore do not 


\begin{tabular}{ccccc} 
Interaction & Mediator & Range $[\mathrm{fm}]$ & Typical Lifetime $[\mathrm{s}]$ & Coupling Strength \\
\hline Electromagnetic & $\gamma$ & $\infty$ & $10^{-19}$ & $\alpha=1 / 137$ \\
Weak & $W^{ \pm}, Z$ & $\sim 10^{-3}$ & $10^{-11}$ & $\alpha_{W}=10^{-6}$ \\
Strong & gluon & $\sim 1$ & $10^{-23}-10^{-24}$ & $\alpha_{s} \sim 1$ \\
Gravity & graviton? & $\infty$ & - & $\alpha_{g}=10^{-39}$
\end{tabular}

Table 2.2: Properties of the fundamental forces of the Standard Model [11].

directly interact among themselves. The $W^{+}, W^{-}$, and $Z$ bosons are massive particles which mediate the weak force. The $Z$ boson is electrically neutral and slightly more massive than the electrically charged $W$ bosons. Together, the $\gamma, W^{ \pm}$, and $Z$ are classified as the electroweak force mediators. Massless gluons mediate the strong force. Unlike the photon, gluons are self-interacting, as they have color charge. It has been theorized that gravity is mediated by an electrically neutral, spin-2 graviton, but such a particle has yet to be discovered. In 2012, a spin-0, electrically neutral particle called the Higgs boson was discovered. Through interactions with the Higgs, elementary particles acquire mass. The Higgs is a massive particle and therefore interacts with itself. In the next section, the properties of the fundamental forces are described in more detail.

\subsubsection{The Fundamental Forces}

\section{The Electromagnetic Force}

Electromagnetic interactions occur between electrically charged particles via the exchange of photons. The related quantum field theory is called quantum electrodynamics (QED). The strength of QED interactions is represented by a dimensionless coupling constant $\alpha$, called the fine structure constant. It is determined by comparing the electron rest mass with the electrostatic energy between two electrons, which are separated by one natural unit of length:

$$
\alpha=\frac{1}{4 \pi \epsilon_{0}} \frac{e^{2}}{\hbar / m_{e} c} / m_{e} c^{2}=\frac{e^{2}}{4 \pi \epsilon_{0} \hbar c} \approx \frac{1}{137}
$$


where $\epsilon_{0}$ is the permittivity of free space, $e$ is the elementary charge, $m_{e}$ is the mass of an electron, $\hbar=h / 2 \pi$ is the reduced Planck constant, and $c$ is the speed of light in vacuum [12. As with the coupling constants of the other fundamental forces, $\alpha$ is dependent on the four-momentum transfer, or $Q^{2}$, that occurs in the interaction:

$$
\alpha\left(Q^{2}\right)=\frac{\alpha\left(\mu^{2}\right)}{1-z_{f} \frac{\alpha\left(\mu^{2}\right)}{3 \pi} \ln \left(|Q|^{2} / \mu^{2}\right)},
$$

where $\mu$ is a scale constant and $z_{f}$ is the sum of the squares of the charges of the fermions with mass $<Q$ [6]. As the energy $Q$ reaches a given fermion mass threshold, particle-antiparticle pairs of that type are resolved and contribute to the value of $\alpha$. While $Q$ denotes the energy of the interaction, $Q^{2}$ is the relevant Lorentz-invariant quantity. This energy dependence can be seen in Fig. 2.1.1. The fine structure constant increases with increasing energy, for example, from $\sim 1 / 137$ at low energies to $\sim 1 / 129$ at energies equal to the $\mathrm{Z}$ mass. The coupling constant is the main factor that sets the scale of the lifetime of a particle decaying via a given interaction [11]. Particles that decay by the electromagnetic interaction have a typical lifetime of $\sim 10^{-19} \mathrm{~s}$. Table 2.2 compares this with the decay lifetimes associated with the other fundamental forces.

The Heisenberg uncertainty principle, $\Delta x \Delta p \geq \hbar / 2$, tells us that the range of a force is inversely proportional to the mass of the associated mediator [6]. Since the photon is massless, the electromagnetic force has infinite range, though its strength falls off as $\sim 1 / r^{2}$. The electromagnetic force is responsible for many bound states found in nature, most notably the bonds between electrons and nuclei that form atoms and molecules. 


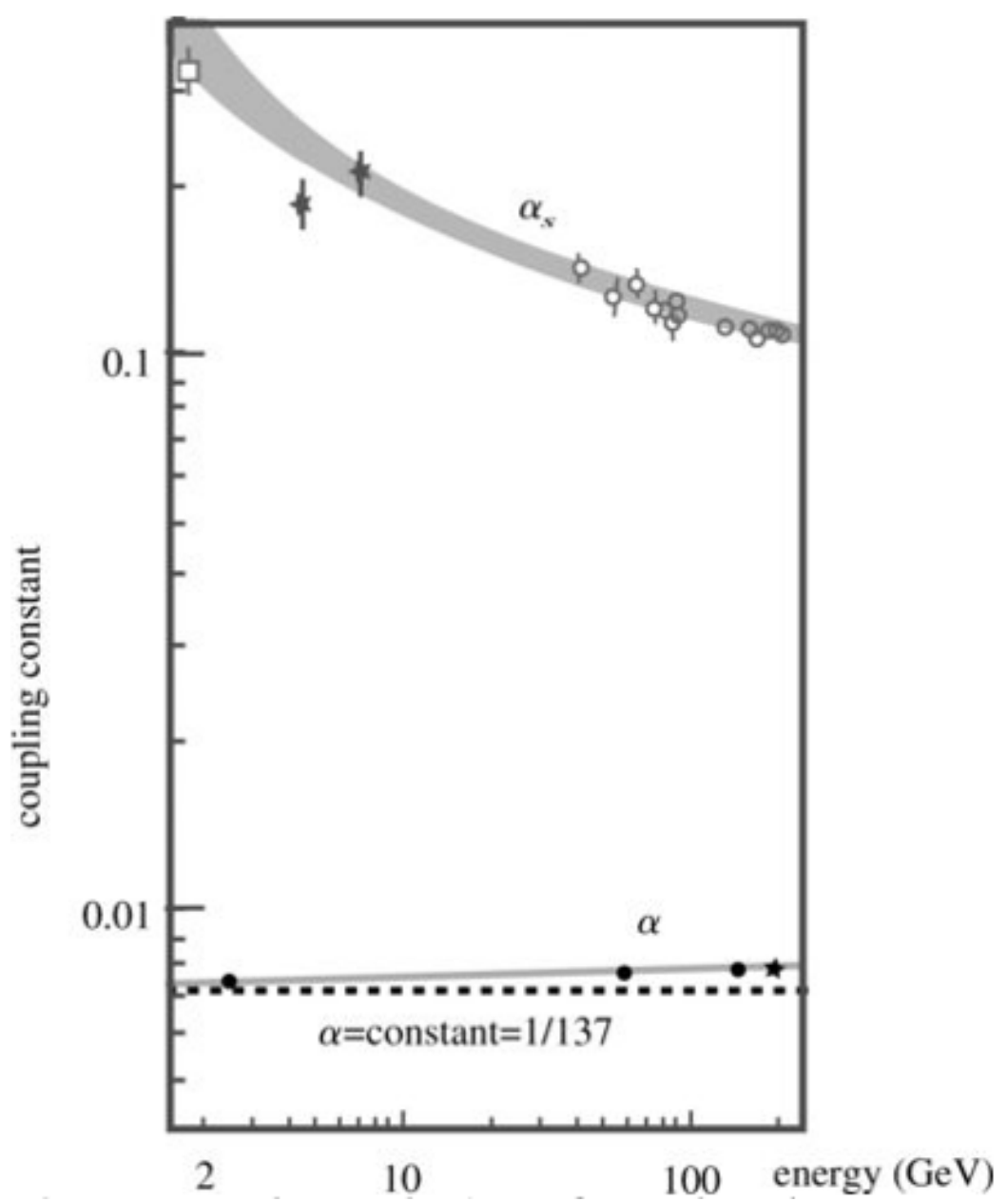

Figure 2.1.1: The strong $\left(\alpha_{s}\right)$ and electromagnetic $(\alpha)$ coupling constants as a function of energy [6].

\section{The Strong Force}

The strong interaction is mediated by massless gluons and is described by quantum chromodynamics (QCD) in the framework of QFT. Unlike in QED, there are three different charges in QCD, known as color charges: red, green, and blue [6]. The only fermions that interact strongly are the quarks. A quark carries a color charge independent of its flavor; likewise antiquarks carry anticolor. The anticolors (antired, antiblue, antigreen) are analogous to negative electric charge in QED. There are eight different gluons that exist, which each carry a different combination of color and anticolor charge. Therefore, gluons interact strongly 
with each other (and with quarks), and do not interact weakly or electromagnetically.

The strong coupling constant is, unsurprisingly, much stronger than those of the electromagnetic or weak forces - at energies of $\sim 1 \mathrm{GeV}, \alpha_{s} \approx 1$ [6]. Therefore, typical lifetimes of particles decaying strongly are quite short, $\sim 10^{-23}-10^{-24} \mathrm{~s}$. Unlike $\alpha, \alpha_{s}$ decreases with energy. Figure 2.1.1 compares the dependence of the two coupling constants on $Q$. This property of $\alpha_{s}$ leads to some interesting features of QCD. If we consider a free quark, its effective color charge grows indefinitely with increasing distance, problematically requiring infinite energy. This can be avoided if the quark exists in a color neutral state, either with an antiquark with opposite color or with two other quarks of different colors than the first. Therefore, in a phenomenon known as color confinement, quarks cannot exist alone, but instead group together to form colorless particles known as hadrons.

Hadrons are either mesons, composed of quark-antiquark pairs, or baryons, composed of three quarks. Protons (uud) and neutrons $(u d d)$ are the two baryons most commonly found in nature. While free neutrons are unstable, protons, on the other hand, are stable. This is due to the fact that proton decay would violate baryon number ( $N$ (baryons) $-N$ (antibaryons)), a quantity conserved in all known interactions. Experiments testing for the most likely proton decay scenario, in which the proton decays into a positron and a neutral $\pi$ meson, have excluded proton lifetimes below $\sim 10^{34}$ years, $10^{24}$ times the age of the universe [6].

As quarks are pulled apart in high energy collisions, the color field energy density between them remains constant, causing the associated energy to increase proportionally with distance. When the energy between a pair of quarks becomes larger than the mass energy of a quark-antiquark pair, it becomes energetically favorable for the original quark pair to radiate a gluon, which in turn produces the new quark-antiquark pair [6]. As more and more quarks are produced, they group into hadrons. This process is known as hadronization and results in a cone of particles known as a jet. Typically, jets formed by light quarks contain mostly $\pi$ and $K$ mesons, while the hadronization of $\mathrm{b}$ quarks will create $\mathrm{B}$ mesons. It is through the observation of jets that quarks are identified in particle detectors. 
The strong force not only binds quarks together to form hadrons, but also binds protons and neutrons together to form atomic nuclei. This nuclear force is also known as the residual strong force and is mediated by $\pi$ mesons, or pions. Due to the mass of the pions, the strong force is not infinite but rather has a range of only $1 \mathrm{fm}$.

\section{The Weak Force}

The weak force is mediated by the electrically charged $80.385 \pm 0.015 \mathrm{GeV} W^{+}$and $W^{-}$ bosons and the $91.1876 \pm 0.0021 \mathrm{GeV}$ neutral $Z$ boson [10]. "Charged-current" (CC) interactions are mediated by a $W$ boson, and the fermions involved differ by one unit of electric charge. "Neutral-current" (NC) interactions involve two fermions with the same electric charge and are mediated by a $Z$ boson [6]. Quark flavor, or type, is conserved in NC, but not CC, processes. Both types of weak processes conserve total lepton number ( $N$ (leptons)$N$ (antileptons)), as the SM does not allow any violation of this quantity [6]. Additionally,

all processes predicted by the Standard Model conserve partial lepton number, e.g. electron flavor number.

The weak charge of a fermion depends on a property called chirality. A particle is said to be "right-handed" if it has positive chirality or "left-handed" if it has negative chirality. Electrons and positrons can be left- or right-handed, but only left-handed (right-handed) neutrinos (anti-neutrinos) exist [6]. $W$ bosons couple only to left-handed particles and right-handed antiparticles, while $Z$ bosons couple to left- and right-handed particles and antiparticles. The $W$ and $Z$ bosons have weak charge and are therefore self-interacting.

As suggested by its name, the coupling strength of the weak force is much smaller than that of the strong or electromagnetic interactions: $\alpha_{W} \approx 10^{-6}$ [11]. Therefore, typical lifetimes of particles decaying via the weak interaction $\left(\sim 10^{-11} \mathrm{~s}\right)$ are much greater than those associated with the other forces. One exception is the top quark. Due to its very large mass, the lifetime of the top quark is only $\sim 10^{-25} \mathrm{~s}$. Because this is shorter than the time scale of hadronization $\left(\sim 10^{-23} \mathrm{~s}\right)$, nearly $100 \%$ of the time, the top quark decays via 
the weak force into a $W, b$ pair before it can hadronize. This property is very useful for the analysis presented in this dissertation.

Due to the massive nature of the $W$ and $Z$ bosons, the weak force extends only to ranges of $\sim 10^{-3} \mathrm{fm}\left(10^{-18} \mathrm{~m}\right)$. Both the weakness of its coupling and the short range over which it interacts contribute to the fact that the weak force is the only fundamental force that does not create bound states. [6].

\section{The Higgs Boson and Electroweak Symmetry Breaking}

The mathematical description of the Standard Model comprises all of the fundamental forces except gravity. This SM QFT can be separated into two independent theories: QCD, based on the symmetry group $\mathrm{SU}(3)_{(\mathrm{C})}$, and electroweak theory (QED), based on the symmetry group $\mathrm{SU}(2)_{(\mathrm{L})} \times \mathrm{U}(1)_{(\mathrm{Y})}$. [13]. The gauge fields associated with the electroweak sector are $B_{\mu}$, the generator of the $\mathrm{U}(1)_{(\mathrm{Y})}$ group, and $W_{\mu}^{1,2,3}$, the three generators of the $\mathrm{SU}(2)_{(\mathrm{L})}$ group.

Without mass terms for the fermions and gauge bosons, the SM Lagrangian is invariant under local $\mathrm{SU}(3)_{(\mathrm{C})} \times \mathrm{SU}(2)_{(\mathrm{L})} \times \mathrm{U}(1)_{(\mathrm{Y})}$ gauge transformations for the fermion and gauge fields [13]. In the case of the strong sector, gluons are indeed massless. Additionally, mass terms can be introduced for the quarks and leptons without compromising the SU(3) gauge invariance of the Lagrangian. Unfortunately, adding mass terms for the fermions and QED gauge bosons violates local $\mathrm{SU}(2)_{(\mathrm{L})} \times \mathrm{U}(1)_{(\mathrm{Y})}$ invariance.

In 1964, three independent groups of physicists introduced a mechanism (now known as the Higgs mechanism) to generate masses for the fermions and gauge bosons without breaking $\mathrm{SU}(2)_{(\mathrm{L})} \times \mathrm{U}(1)_{(\mathrm{Y})}$ gauge invariance. In this theory, a scalar field $\phi$ is introduced, with a potential $V(\phi)=\frac{1}{2} \mu^{2} \phi^{2}+\frac{1}{4} \lambda \phi^{4}$ [13], in which $\mu^{2}$ is a mass squared term, and $\lambda$ is the (positive) self-coupling of the field. A corresponding Lagrangian is also introduced, $\mathcal{L}=\frac{1}{2} \partial_{\mu} \phi \partial^{\mu} \phi-V(\phi)$, which is symmetric under $\phi \rightarrow-\phi$. 

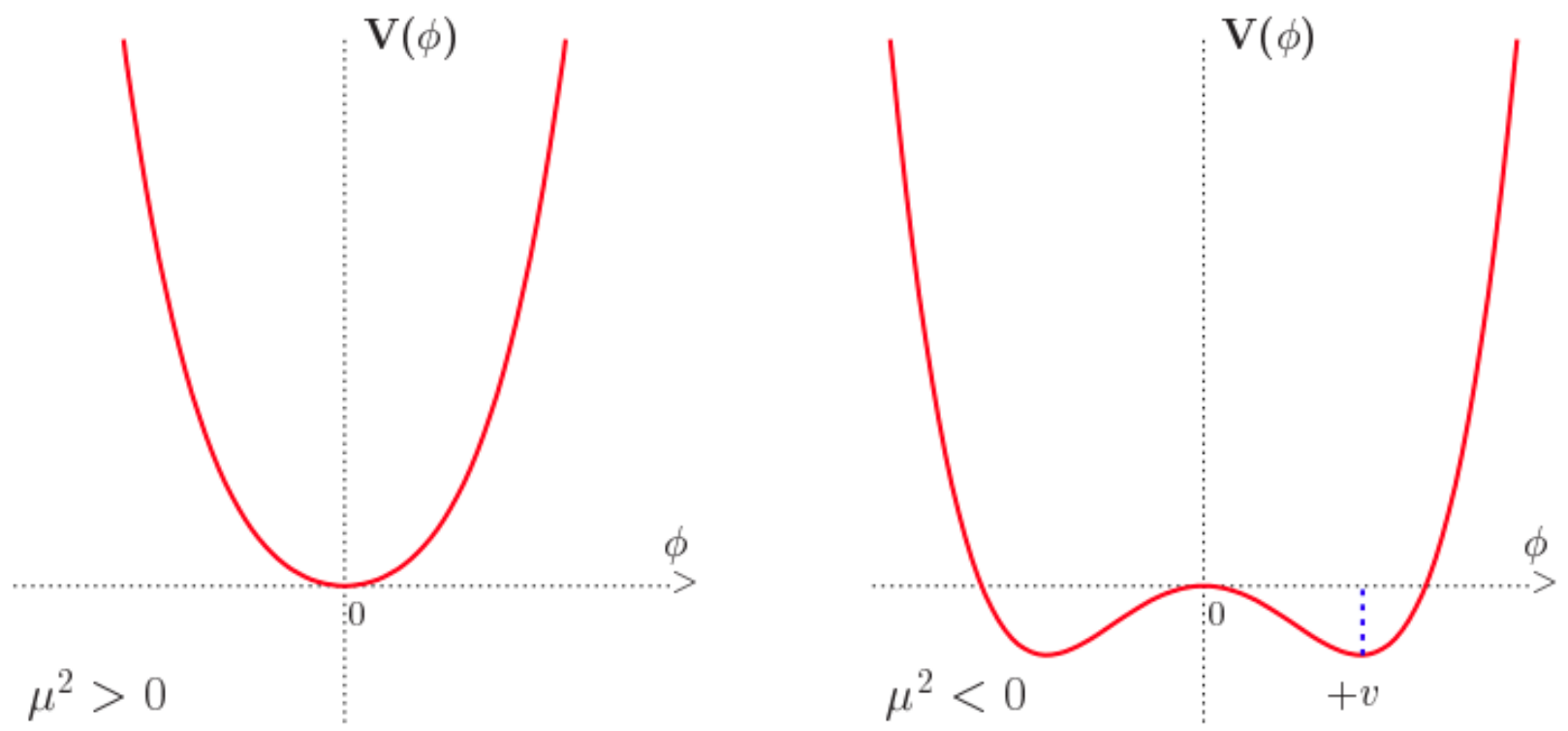

Figure 2.1.2: The potential $V(\phi)$ for $\mu^{2}>0$ (left) and $\mu^{2}<0$ (right) [13].

Figure 2.1.2 shows the potential $V$, considering two possible cases for $\mu^{2}: \mu^{2}>0$ and $\mu^{2}<0$. In the latter case, $\phi$ acquires a vacuum expectation value (vev) of $v$, where $v$ is defined as $v^{2} \equiv-\frac{\mu^{2}}{\lambda}$, located at one of the many nonzero minima of $V$. The choice of a particular vev, and the subsequent expansion around this minimum, introduces a cubic term into the Lagrangian, breaking the previously stated symmetry. This is also known as spontaneous symmetry breaking [13].

The simplest case of spontaneous electroweak symmetry breaking requires a scalar field doublet $\phi$, which has a neutral component $\phi^{0}$ and a charged component $\phi^{+}$[13]. Combining the original SM Lagrangian with the scalar field Lagrangian and requiring $\mu^{2}<0$, causes $\phi^{0}$ to acquire a vev. According to the Goldstone theorem, every spontaneously broken continuous symmetry has a corresponding massless scalar particle (spin-0) called a Goldstone boson. For each broken generator, there is one Goldstone boson. In accordance with this theory, an expansion around the $\phi^{0}$ vev introduces new Goldstone bosons into the Lagrangian.

An appropriate gauge transformation produces a Lagrangian with terms that are quadratic 
in $W_{\mu}^{1,2,3}$ and $B_{\mu}$, the mass terms of the QED generators. During the course of the gauge transformation, the Goldstone bosons are absorbed into the longitudinal degrees of freedom of the $W_{\mu}^{1,2,3}$ fields. Expressing the $W \pm$ bosons as linear combinations of $W_{\mu}^{1}$ and $W_{\mu}^{2}$ and the $Z$ boson and photon as linear combinations of $W_{\mu}^{3}$ and $B_{\mu}$ results in massive $W \pm$ and $Z$ bosons and a massless photon. Therefore, the electroweak symmetry breaking of the Higgs mechanism provides boson masses consistent with experimental results while maintaining $\mathrm{SU}(2)_{(\mathrm{L})} \times \mathrm{U}(1)_{(\mathrm{Y})}$ symmetry. Additionally, $\mathrm{SU}(2)_{(\mathrm{L})} \times \mathrm{U}(1)_{(\mathrm{Y})}$ invariant Yukawa interactions of the same scalar Higgs field generate the masses of the fermions.

In 2012, 48 years after its existence was theorized, the Higgs boson (the excitation of the Higgs field) was discovered by the CMS and ATLAS experiments at the LHC, with a mass of $125.09 \pm 0.24 \mathrm{GeV}$ [14, 15, 10]. With this discovery, the Standard Model was complete all of the elementary particles it predicted had been found.

\section{Gravity}

The gravitational interaction is felt as an attractive force between massive particles. It is the only one of the four fundamental forces whose mediator has not been discovered. While no complete QFT description of gravity exists, an effective coupling constant can be determined for the sake of comparison with the other three forces [11]. Similarly to $\alpha$, one can use the gravitational attraction between two protons to calculate $\alpha_{g}$ :

$$
\alpha_{g}=\frac{G m_{p}^{2}}{\hbar c} \approx 10^{-39}
$$

where $G$ is Newton's gravitational constant. Comparing $\alpha_{g}$ with the other coupling constants, we can see that gravity is the weakest of the fundamental forces by at least 33 orders of magnitude. Despite its weakness, gravity is the dominant force over large scales. Similarly to the electric force, gravity has an infinite range (falling off at a rate of $1 / r^{2}$ ). However, in contrast with electromagnetism, there is no negative mass to screen the attractive force felt between massive particles. The large-scale nature of gravity leads to bound states such as 
solar systems and galaxies.

\subsection{Beyond the Standard Model}

\subsubsection{Standard Model Limitations}

Although the Standard Model successfully describes a large portion of the experimental observations in particle physics, it fails to answer certain key questions. Some of the limitations of the Standard Model are listed below [16]:

- The Fermion Problem: The matter we observe on a day-to-day basis is composed of first-generation fermions: electrons, electron neutrinos, up quarks, and down quarks. However, experiments have shown that there exist two additional heavier generations of fermions. The Standard Model does not explain the number of fermion families, nor does it predict the fermion masses. Additionally, the SM does not provide an explanation for the fact that our universe is dominated by matter, not antimatter, fermions.

- Dark Matter and Dark Energy: From cosmological observations, we know that ordinary baryonic matter makes up only $4-5 \%$ of the mass-energy content of the universe. Measurements of galaxy rotation curves and galaxy cluster masses indicate that a type of massive, nonelectromagnetically interacting type of matter, called dark matter, makes up about $25 \%$ of the universe. Additionally, it has been observed that the universe is expanding at an accelerating rate. If the universe was only made up of matter, gravity should have caused its rate of expansion to decrease. Measurements of the universal expansion rate indicate that the rest of the universe is in fact composed of dark energy, a density of negative pressure causing this accelerated expansion [6]. While the Standard Model provides a good description of baryonic matter, it does not account for dark matter or dark energy - $95 \%$ of the total content of the universe! 
- Gravity: There are four known fundamental forces in particle physics: electromagnetism, the strong force, the weak force, and gravity. The SM provides a solid theoretical description of the former three forces, along with the associated mediator particles. Gravity, the weakest force, is well described by the theory of general relativity. In the classical limit, the force of gravity can be described in the framework of the Standard Model, with gravitational interactions mediated by virtual gravitons. However, this model breaks down at high energies $\left(\sim 10^{19} \mathrm{GeV}\right)$ and short distances $\left(\sim 10^{-35} \mathrm{~m}\right)$, known respectively as the Planck energy and Planck length. This indicates the need for a theory of quantum gravity that goes beyond the Standard Model.

- The Hierarchy Problem: Another shortcoming of the SM is that it does not explain the large difference between the $\sim 10^{19} \mathrm{GeV}$ Planck scale and $\sim 100 \mathrm{GeV}$ electroweak scale. The mass of the Higgs boson is an important manifestation of this problem. The Higgs mass can be approximated as $m_{H}^{2} \approx m_{\text {bare }}^{2}+\Delta m_{H}^{2}$, where $m_{H}$ is the mass that is measured, $m_{\text {bare }}^{2}$ is a negative free parameter, and $\Delta m_{H}$ is the contribution from quantum corrections. The largest component of the second term is $\frac{3}{8 \pi^{2}} y_{t}^{2} \Lambda^{2}$, where $y_{t}$ is the coupling strength of the Higgs to the top quark, the most massive elementary particle, and $\Lambda$ is the scale at which new physics beyond the standard model (BSM) becomes relevant. If there is no new physics until the Planck scale, the second term of the Higgs mass equation indicates that the Higgs mass should be much greater than $125 \mathrm{GeV}$, unless there is a very fine tuning of the bare mass parameter. This is known as the hierarchy problem.

In order to solve these problems, many extensions to the Standard Model have been proposed. Some of these theories motivate the existence of new particles or extra dimensions, which often predict new physics at the TeV scale. 


\subsubsection{Heavy $t \bar{t}$ Resonances}

A proposed solution to the hierarchy problem, the Randall-Sundrum RS1 model suggests that our universe contains a warped extra dimension, in addition to the four known space-time dimensions [17]. In this five-dimensional model, there exists a flat four-dimensional Planck brane separated from a four-dimensional $\mathrm{TeV}$ brane by a finite extra dimension. The $\mathrm{TeV}$ brane contains the Standard Model particles and the electroweak forces. There also exists a five-dimensional graviton, which has an essentially four-dimensional bound-state mode that is concentrated on the Planck brane. Due to the warped nature of the fifth dimension, the strength of gravity decreases exponentially as a function of distance from the Planck brane. This accounts for the apparent weakness of gravity that we observe on the $\mathrm{TeV}$ brane and is the reason for the large difference in energy scales.

Unfortunately, the RS1 model predicts rates of flavor changing neutral currents and contributions to SM electroweak precision test observables inconsistent with experimental measurements [18]. A solution to this problem is to allow not only gravity, but also the SM fields, to propagate in the fifth dimension. The SM particles can then be described as the zero-modes of these five dimensional fields, analogous to the lowest order normal mode of a harmonic oscillator. The first and second generation fermions are localized near the Planck brane, while the Higgs boson and top quark are localized near the TeV brane. This suppresses the large RS1 contributions to flavor changing neutral currents and SM electroweak precision

test observables. Additionally, it helps to explain the hierarchy of fermion masses, as the fermions localized farther from the Higgs have a smaller Yukawa coupling.

The higher order modes of the five dimensional SM fields give rise to four dimensional Kaluza-Klein (KK) partners of the SM particles. In this extended RS1 model, the gauge KK modes are predicted to be localized near the TeV brane. They are therefore expected to be massive and have large couplings to the top quark [18]. Of the gauge KK particles, the KK gluon is predicted to have the largest production rate at the LHC. Its production cross section, proportional to the probability it will be produced, is shown on the left in Fig. 
2.2.1, assuming proton-proton collisions with a $14 \mathrm{TeV}$ center-of-mass energy. It is expected to be detectable up to a mass of $5 \mathrm{TeV}$. Massive KK gluons will decay to $t \bar{t}$ pairs $95 \%$ of the time, as shown in on the right in Fig. 2.2.1.
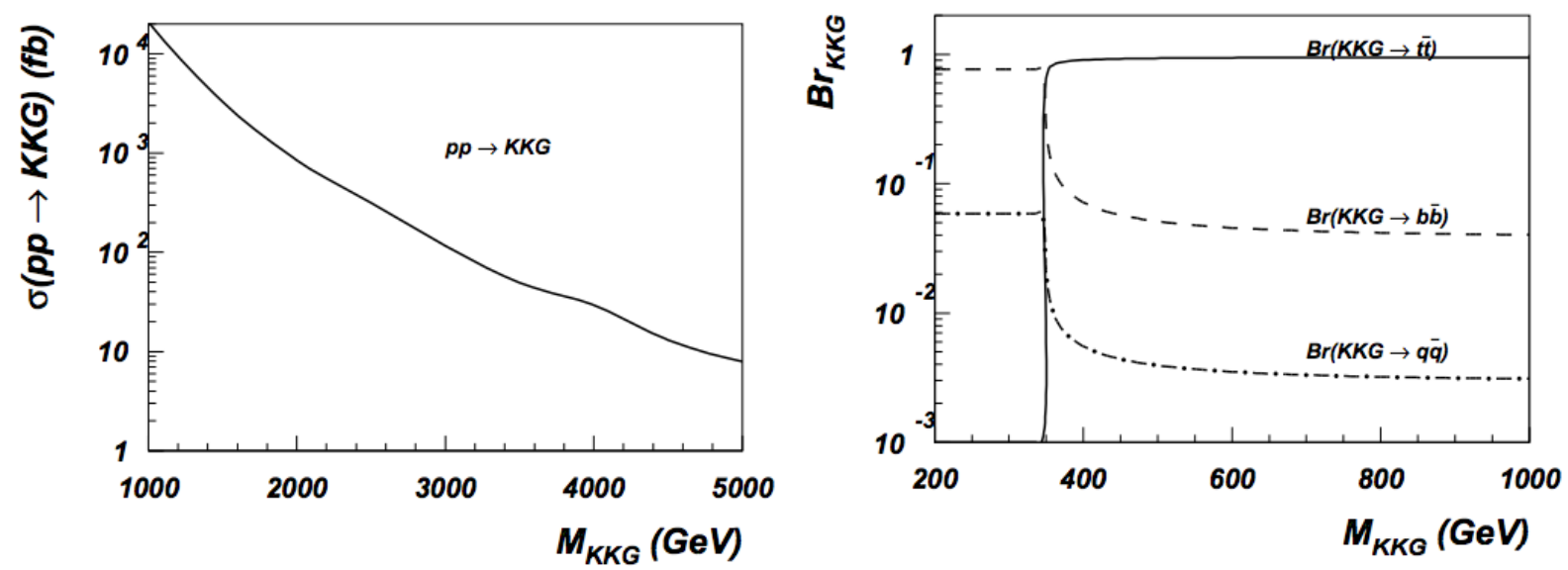

Figure 2.2.1: Left: The cross section of a KK gluon as a function of its mass, assuming 14 TeV proton-proton collisions. Right: The branching ratio of a KK gluon as a function of its mass. Massive RS KK gluons decay mainly to top quark pairs [18].

Many other models of new physics also motivate the existence of new heavy resonances that decay primarily to $t \bar{t}$ pairs, generically called leptophobic topcolor $Z^{\prime}$ gauge bosons [19, 20, 21, 22, 23]. In the high mass ranges accessible by the LHC at $\sqrt{s}=13 \mathrm{TeV}, \mathrm{RS} \mathrm{KK}$ gluons and $Z^{\prime}$ bosons would produce highly Lorentz-boosted top quark pairs. The subsequent decay products of each top quark would merge together. The search for such a unique event topology is the focus of this dissertation. 


\section{Chapter 3}

\section{Experimental Apparatus}

The analysis presented in this dissertation uses $13 \mathrm{TeV}$ proton-proton collision data taken by the Compact Muon Solenoid (CMS) detector at the Large Hadron Collider (LHC) at CERN. This chapter introduces the LHC and the component systems of the CMS detector. Further information about the LHC and CMS can be found in Refs. [24] and [25].

\subsection{The Large Hadron Collider}

Located on the Franco-Swiss border near Geneva, Switzerland, the LHC is the largest and most powerful particle accelerator in the world. It consists of two interleaved rings, $27 \mathrm{~km}$ in circumference, which are used to accelerate protons and lead ions. The accelerator is located between $45 \mathrm{~m}$ and $170 \mathrm{~m}$ underground, at a slope of $1.4 \%$. While the depth of the LHC tunnel was chosen for geological and cost reasons, the layers of earth above the experiments also provide useful shielding from radiation.

The proton-proton collision process starts with a bottle of hydrogen, from which hydrogen atoms are fed into the source chamber of the LINAC2 linear accelerator. Using an electric field, the atoms are stripped of their electrons, leaving behind hydrogen nuclei: protons. Next, an electric field is used to accelerate the protons through the LINAC2, at the end of which they have an energy of $50 \mathrm{MeV}$. The protons are then injected into the first circular 


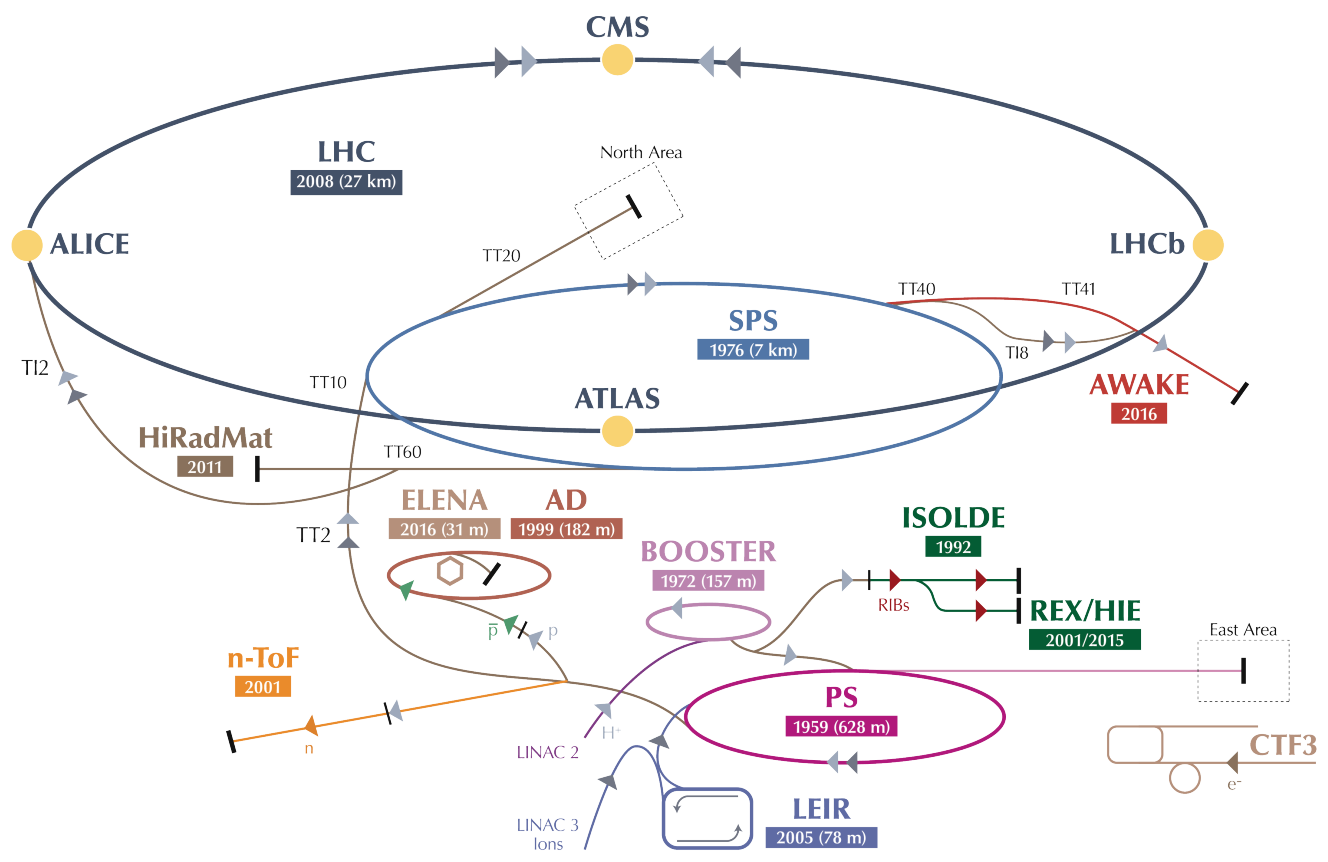

$\rightarrow \mathrm{p}$ (protons)

$\rightarrow$ ions

RIBs (Radioactive lon Beams)

n (neutrons)

$\overline{\mathrm{p}}$ (antiprotons)

- é (electrons)

$\rightarrow+\rightarrow$ proton/antiproton conversion

$\rightarrow+$ proton/RIB conversio

LHC Large Hadron Collider SPS Super Proton Synchrotron PS Proton Synchrotron AD Antiproton Decelerator CTF3 Clic Test Facility

AWAKE Advanced WAKefield Experiment ISOLDE Isotope Separator OnLine REX/HIE Radioactive EXperiment/High Intensity and Energy ISOLDE

LEIR Low Energy lon Ring LINAC LINear ACcelerator n-ToF Neutrons Time Of Flight HiRadMat High-Radiation to Materials

Figure 3.1.1: The CERN accelerator complex. 
accelerator in the chain: the Proton Synchrotron Booster (PSB), which comprises four 157m-circumference rings. The PSB outputs $1.4 \mathrm{GeV}$ protons, which are then accelerated to $25 \mathrm{GeV}$ by the circular Proton Synchroton (PS). The last stage before the LHC, the Super Proton Synchrotron (SPS), accelerates the protons to $450 \mathrm{GeV}$. This acceleration chain is illustrated in Fig. 3.1.1.

The protons are separated into two beams, which are circulated in opposite directions in the two rings of the LHC. In the 2011-2012 data-taking period of the LHC (Run I), beams were circulated at energies of $3.5 \mathrm{TeV}$ (starting in 2011) and $4 \mathrm{TeV}$ (starting in 2012). Now in Run II, the LHC accelerates each beam to an energy of $6.5 \mathrm{TeV}$, though it was designed to accelerate protons up to $7 \mathrm{TeV}$. To maintain the beams' circular trajectories, the LHC contains 9593 magnets, most notably the superconducting dipole magnets, used to bend the beams and the quadrupole magnets, used to focus the beams.

Each beam consists of 2808 bunches, which in turn starts out with $1.2 \times 10^{11}$ protons. The bunches are $7.55 \mathrm{~cm}$ long and $16.6 \mu \mathrm{m}$ wide. Eight radio frequency $(\mathrm{RF})$ cavities are used to keep the protons tightly bunched and at a constant energy. The bunches circulate 11,245 times per second and are separated by approximately $7.5 \mathrm{~m}$.

Every $25 \mathrm{~ns}$, the two counter-rotating beams collide in four experimental caverns. Since multiple protons collide per bunch crossing, approximately 1 billion collisions occur every second. The four LHC experiments are CMS, ATLAS, ALICE, and LHCb. CMS and ATLAS are multipurpose experiments, which study both proton and lead-ion collisions. ALICE studies the quark-gluon plasma created in lead-ion collisions, while LHCb's primary focus is the matter/antimatter asymmetry present in the interactions of particles containing b quarks. The experimental focus of this dissertation is the CMS detector, which will be described in further detail later in this chapter. 


\subsubsection{High Luminosity LHC}

LHC performance is measured in luminosity, which is defined as the number of collisions per unit time per cross sectional area. The number of generated events is described by the equation $N_{\text {event }}=L \sigma_{\text {event }}$, where $L$ is the luminosity and $\sigma_{\text {event }}$ is the cross section for the process of interest. The LHC has a design luminosity of $10^{34} \mathrm{~cm}^{-2} \mathrm{~s}^{-1}[24$.

In order to extend the discovery reach of the LHC, in June 2014 the CERN Council approved funding of the High Luminosity LHC (HL-LHC). Additionally, the 2014 report of the United States' Particle Physics Project Prioritization Panel (P5) supported investment in the project. In the 2020s, major upgrades will be made to the LHC in order to increase the luminosity by a factor of five, compared to the design value [26]. Figure 3.1.2 shows the LHC's long term luminosity plans through 2038 (as of October 30, 2017) citeHLLHCworkshop. The maximum instantaneous luminosity for each year is shown in red, while the total cumulative, or integrated, luminosity is shown in blue. The grey bands show periods of time called "long shutdowns" when the LHC is off, so that upgrades can be performed on the LHC and the experiments. The detector work described in this dissertation was done to help prepare the CMS muon detectors for the HL-LHC data-taking period. Many HL-LHC detector upgrades are also called "Phase-II" upgrades.

\subsection{The Compact Muon Solenoid}

CMS is a cylindrical multipurpose particle detector which features a 13-m-long, $3.8 \mathrm{~T}$ superconducting solenoidal magnet centered on the particle beam pipe. The powerful magnet was chosen in order to provide large bending power for charged particles, and therefore excellent momentum resolution. Within the $6 \mathrm{~m}$ internal diameter of the magnet are several detector subsystems: silicon pixel and tracker detectors, a lead tungstate electromagnetic calorimeter (ECAL), and a brass-and-scintillator hadronic calorimeter (HCAL). Muon detectors are embedded within the steel magnetic flux-return yoke outside of the magnet volume. 


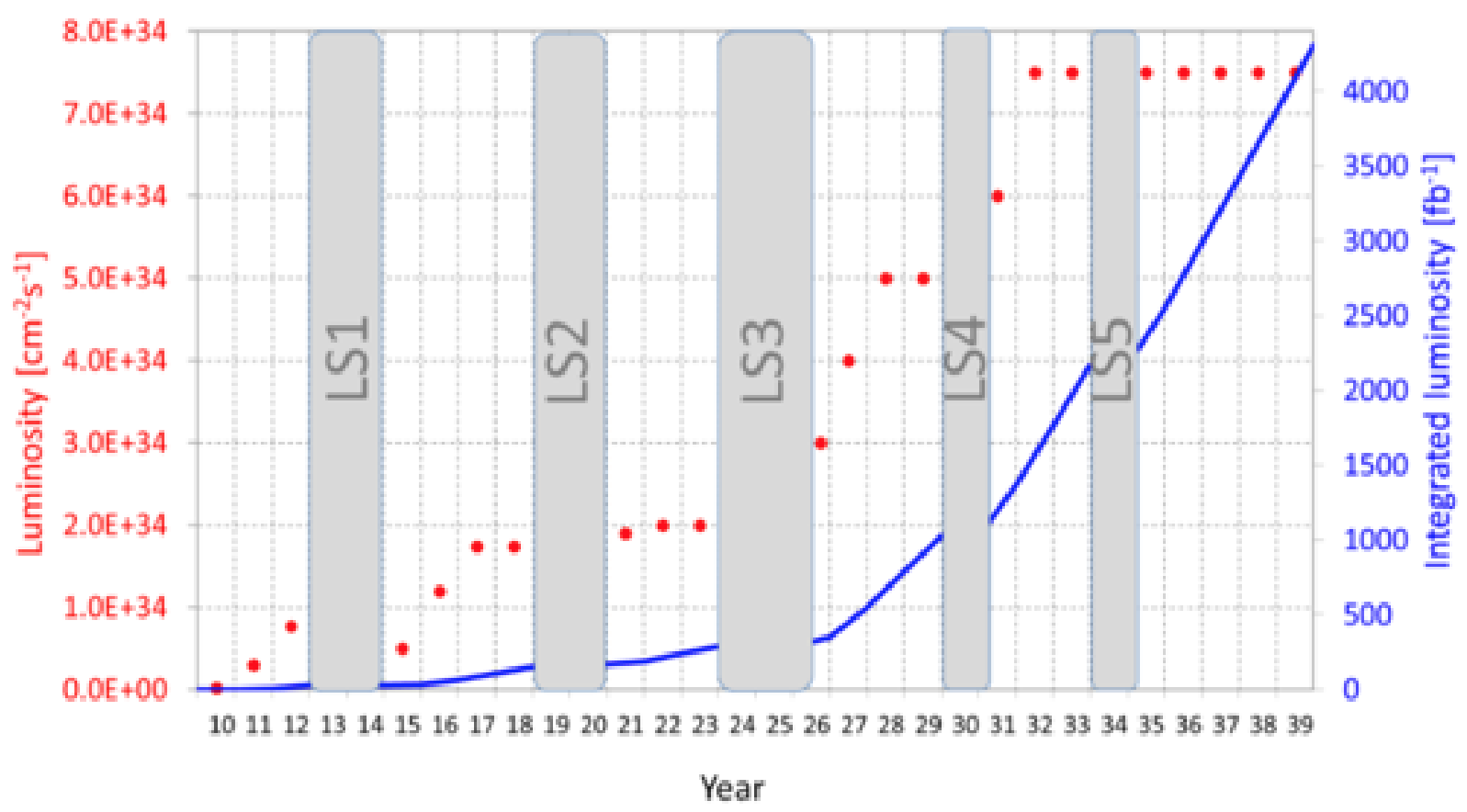

Figure 3.1.2: The planned luminosity of the LHC and HL-HLC through 2038 [27.

A three-dimensional depiction of the CMS detector is shown in Figure 3.2.1. Each subsystem has barrel and endcap components. The barrel detectors are arranged in cylinders around the beam pipe, while the endcap detectors are arranged in disks at the ends of the corresponding barrel detectors. Overall, CMS is $28.7 \mathrm{~m}$ long and $15 \mathrm{~m}$ in diameter, with a total weight of 14,000 t [28]. The CMS magnet and detector subsystems will be described in greater detail later on in this chapter. A more thorough treatment of the CMS detector can be found in the CMS Technical Design Report [29], the CMS Phase II Technical Proposal [28], and the Journal of Instrumentation [25].

\subsubsection{CMS Coordinate System}

The origin of the CMS coordinate system is defined to be at the collision point within the experiment. The $y$-axis points vertically upward, the $x$-axis points radially inward toward the LHC's center, and the $z$-axis points along the beam line toward the Jura mountains [25]. 


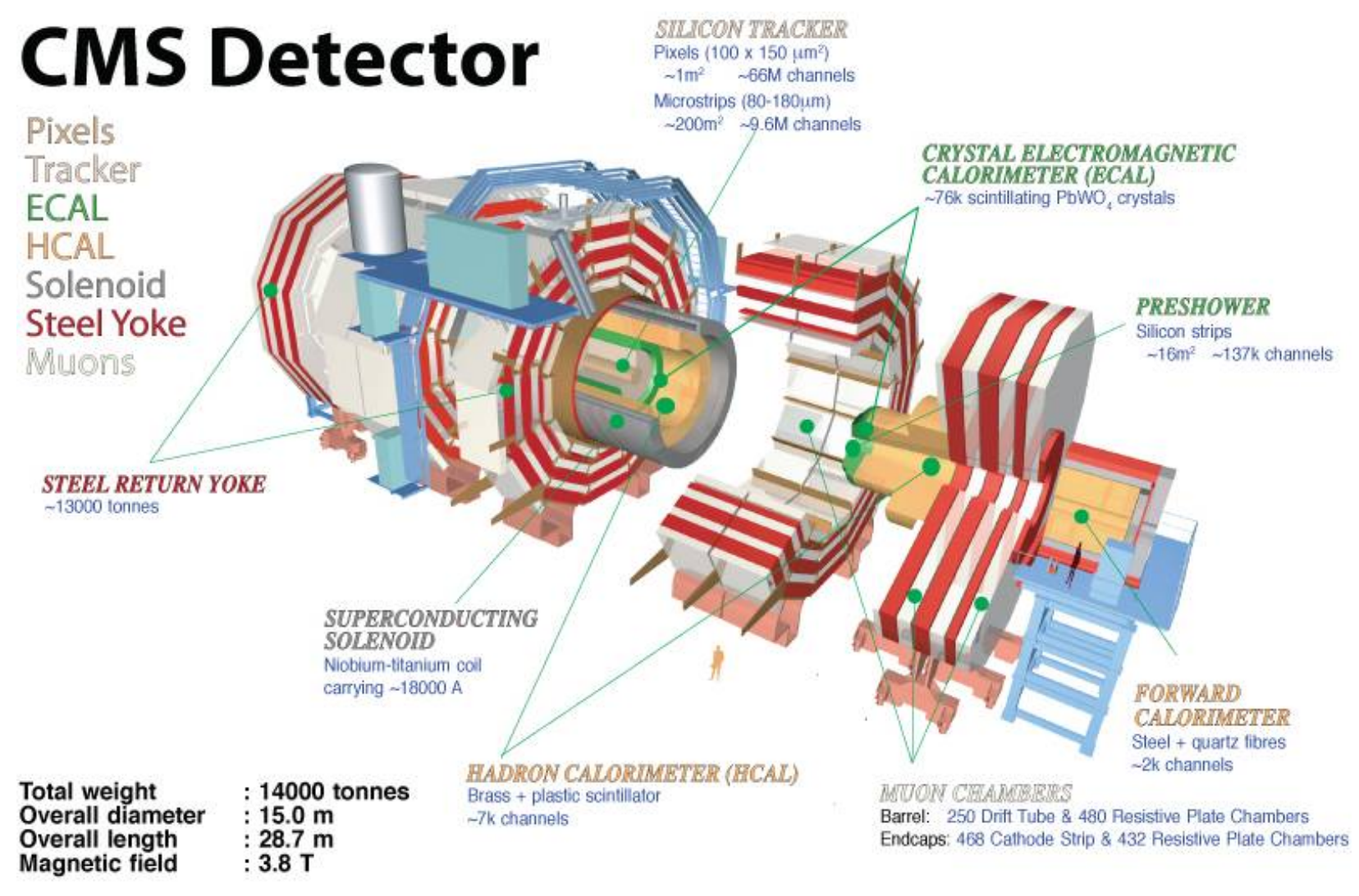

Figure 3.2.1: A 3D schematic view of the Run I CMS detector [28]. 
In the $x-y$ plane, the radial coordinate is defined as $r=\sqrt{x^{2}+y^{2}}$, while the azimuthal angle $\phi$ is the angle measured from the $x$-axis. The polar angle $\theta$ is the angle measured from the $z$-axis.

Instead of $\theta$, the CMS collaboration preferentially uses pseudorapidity, which is defined as $\eta=-\ln [\tan (\theta / 2)]$. For a particle traveling close to the speed of light, at which point its mass is negligible compared to its momentum, pseudorapidity converges to a variable called rapidity:

$$
y=\frac{1}{2} \ln \left(\frac{E+p_{z}}{E-p_{z}}\right)
$$

Rapidity is useful because particle production is more or less constant as a function of $y$. Additionally, rapidity differences $(\Delta y)$ are Lorentz invariant under boosts along the $z$ axis. In high energy events at the LHC, the particles colliding are not whole protons, but rather the partons (quarks and gluons) inside of the protons. Each parton carries a different fraction of the proton's total momentum, characterized by parton distribution functions (PDFs), and therefore the rest frame of each parton-parton collision has a different boost in the $z$-direction.

The variable $\Delta R=\sqrt{(\Delta y)^{2}+(\Delta \phi)^{2}}$ is used to measure the angular separation between particles in CMS. Because $\Delta \phi$ is independent of $z, \Delta R$ is also invariant under boosts along the beam direction.

In CMS, a particle's momentum and energy measured perpendicular to the $z$-direction are denoted by $p_{T}$ and $E_{T}$, respectively. In an LHC collision, colliding particles move only in the beam direction. Therefore the total initial $p_{T}$ and $E_{T}$ are zero. Any final state momentum measured in the in transverse plane would indicate that a particle was either mismeasured or not detected at all. This imbalance of energy in the $x-y$ plane is known as missing transverse energy $\left(E_{T}^{\text {miss }}\right)[25]$. 


\subsubsection{CMS Tracker System}

Surrounding the interaction point (IP), the tracker is the innermost CMS subsystem. It was designed to enable precise measurement of the trajectories of high $p_{T}(>1 \mathrm{GeV})$ charged particles and precise reconstruction of secondary vertices [25]. The nature of LHC collisions, causing thousands of particles to traverse the tracker every $25 \mathrm{~ns}$, introduces unique challenges for an efficient tracker. The high particle flux requires a detector that can operate in a high radiation environment for many years. Additionally, a detector with high granularity and fast response time is called for. Such a system necessitates on-detector electronics, which in turn need proper cooling. The need for physical support systems, as well as electronics and cooling infrastructure, must be balanced with the goal of minimizing material that would increase the rates of multiple scattering, bremsstrahlung radiation, photon conversion, and nuclear interactions.

The many requirements on the CMS tracking system lead to the development of an allsilicon detector. Due to a larger hit rate density closer to the IP and a desire to keep the occupancy at $1 \%$ or less, pixel detectors were chosen to be used below radii of $10 \mathrm{~cm}$. A reduced particle flux further from the beam line allows for the use of silicon strip detectors at larger radii. Figure 3.2 .2 illustrates the layout of the tracking system in CMS. It contains three pixel barrel layers and ten silicon strip barrel layers, as well as 2 pixel disks and 12 strip tracker disks on each endcap. Overall, the CMS tracker is $5.8 \mathrm{~m}$ long, $2.5 \mathrm{~m}$ in diameter, and covers the pseudorapidy range of $|\eta|<2.5$. It is the largest silicon tracker ever built, with a total active silicon area of $\sim 200 \mathrm{~m}^{2}$ [25]. Despite this large area, it manages to minimize material in the $\eta$-direction, having a maximum depth of 1.8 radiation lengths $X_{0}$ at $|\eta| \approx 1.4$.

\section{Pixel Detector}

The pixel detector is the innermost part of the CMS tracker, with $100 \times 150 \mu \mathrm{m}^{2}$ cells in $r-\phi$ and z, respectively [25]. This high granularity allows for small impact parameter resolution $(\sim 15-20 \mu \mathrm{m})$, which is necessary for precise secondary vertex reconstruction. Secondary 


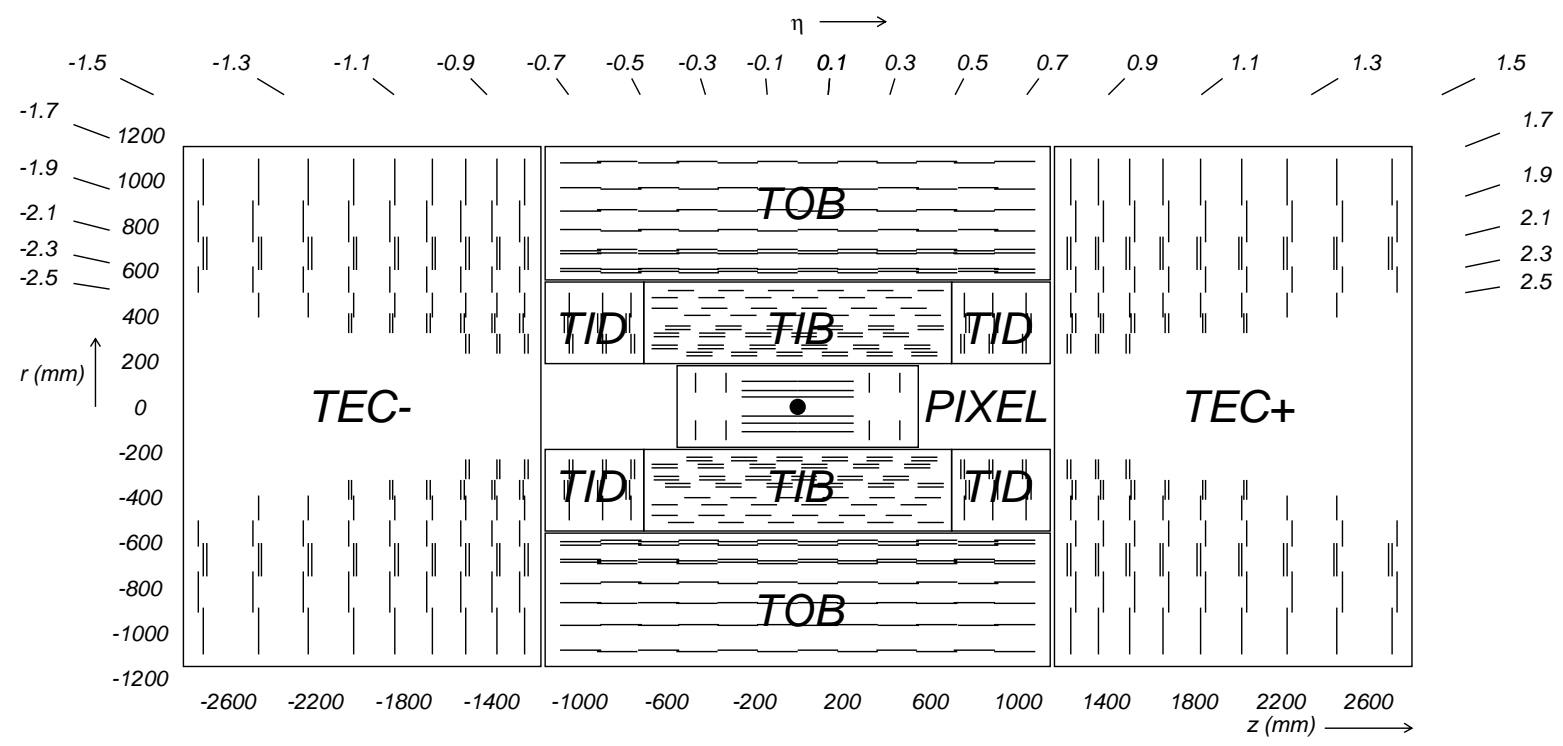

Figure 3.2.2: Cross section of the CMS tracker detector. Each line corresponds to a detector module [25].

vertices are a important aspect of identifying b jets, or b-tagging, which will be discussed further in later chapters.

The pixel detector comprises three barrel layers (BPix) and two endcap disks (FPix), and covers the pseudorapidity range $|\eta|<2.5$. The BPix layers are $53 \mathrm{~cm}$ long and are located at radii of 4.4,7.3, and $10.2 \mathrm{~cm}$. The FPix endcap disks are located at $z= \pm 34.5$ and $z= \pm 46.5 \mathrm{~cm}$, with an inner (outer) radius of $6(15) \mathrm{cm}$. BPix contains 48 million pixels, covering a total area of $0.78 \mathrm{~m}^{2}$, while FPix covers an area of $0.28 \mathrm{~m}^{2}$ and contains 18 million pixels.

The layout of the pixel system provides three tracking points for almost the entire $\eta$-range. In the barrel region, the magnetic field causes a Lorentz drift in the electrons traveling to the collecting pixel implants. This leads to charge sharing and improved spatial resolution. In order to induce charge sharing in the disks, the FPix are tilted at a 20 degree angle in a turbine-like geometry [25]. A three-dimensional illustration of the pixel detector is shown in Fig. 3.2 .3 , 


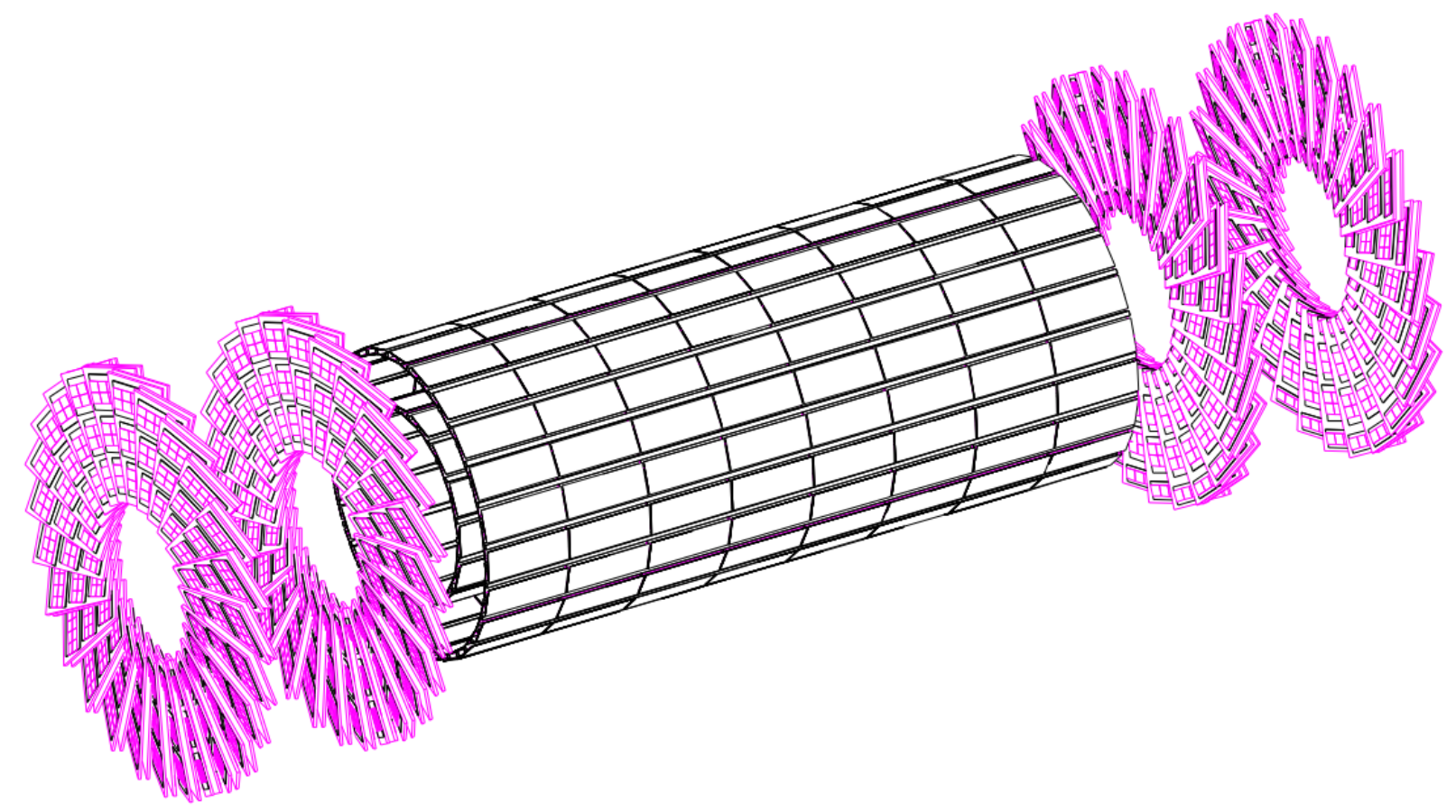

Figure 3.2.3: Three-dimensional depiction of the CMS pixel detector [30].

\section{Silicon Strip Tracker}

Just outside of the pixel detector, the silicon strip tracker (SST) lies in the $20-116 \mathrm{~cm}$ radial region. As shown in Fig. 3.2.2, the SST comprises three different subsystems: the Tracker Inner Barrel and Disks (TIB/TID), the Tracker Outer Barrel (TOB), and the Tracker Endcaps (TEC+ and TEC-). The innermost of these, the TIB/TID is made up of 4 barrel layers and three disks on each endcap, occupying the radial region between 20 and $55 \mathrm{~cm}$ [25]. The TIB/TID subsystem is composed of silicon micro-strip sensors, $320 \mu \mathrm{m}$ thick, which lie parallel to the the beam axis in the barrel region and along the radial direction in the endcap disks. Of the four barrel layers, the inner (outer) two contain strips arranged at a pitch of $80(120) \mu \mathrm{m}$, with a single point resolution of 23 (35) $\mu \mathrm{m}$. The strip pitch in the the TID varies between 100 and $141 \mu \mathrm{m}$. Together, the TIB/TID system provides up to four $r-\phi$ measurements.

Surrounding the TIB/TID is the TOB, with an outer radius of $116 \mathrm{~cm}$ and extending 
between $\pm 118 \mathrm{~cm}$ in $z$. The TOB contains 500 - $\mu$ m-thick micro-strip detectors, arranged in six barrel layers. On the first four layers, the strips have pitches of $183 \mu \mathrm{m}$, while the strip pitches are $122 \mu \mathrm{m}$ on the outer two layers. The TOB delivers an additional $6 r-\phi$ measurements, with a $53(35) \mu \mathrm{m}$ single point resolution on layers 1-4 (5 and 6).

The TEC \pm subsystem is the outermost part of the tracker in the $z$-direction, covering the region $22.5 \mathrm{~cm}<|r|<113.5 \mathrm{~cm}$ and $124 \mathrm{~cm}<|z|<282 \mathrm{~cm}[25]$. Both the TEC+ and TEC-, where the sign indicates the positive or negative $z$-axis, contain nine disks, each with up to seven rings of silicon micro-strip sensors. The strips are 320 (500) $\mu \mathrm{m}$ thick on rings $1-4(5-7)$ and have an average pitch of $97-184 \mu \mathrm{m}$. Each TEC provides up to nine $r-\phi$ measurements for a given particle trajectory.

As indicated by the double lines in Fig. 3.2.2, the modules in rings 1,2 , and 5 of each TEC, as well as the first two layers and rings of TIB/TID and TOB, are mounted back-toback with a second micro-strip detector. This second detector lies at a stereo angle of 100 mrad, providing a measurement of the second coordinate, $\mathrm{r}$ on the disks and $\mathrm{z}$ in the barrel. This provides a single point resolution of 230 (530) $\mu \mathrm{m}$ in the TIB (TOB); the resolution varies with pitch in the disks. The arrangement of the SST subsystems delivers $\sim 9$ hits in the pseudorapidity region $|\eta|<2.4$, at least four of which are two-dimensional measurements [25]. In total, the silicon strip tracker contains 9.3 million strips, 24,244 silicon sensors, and $198 \mathrm{~m}^{2}$ of active silicon area.

\subsubsection{Electromagnetic Calorimeter}

Surrounding the tracker is the CMS electromagnetic calorimeter (ECAL). The ECAL is made of lead tungstate $\left(\mathrm{PbWO}_{4}\right)$ crystals and is designed to make very accurate measurements of electron and photon energies. As with the tracker, the CMS electromagnetic calorimeter is composed of a barrel section (EB) and two endcap disks (EE) [25]. Its layout is shown in Fig. 3.2 .4 .

Lead tungstate crystals have several characteristics that make them ideal for an LHC 


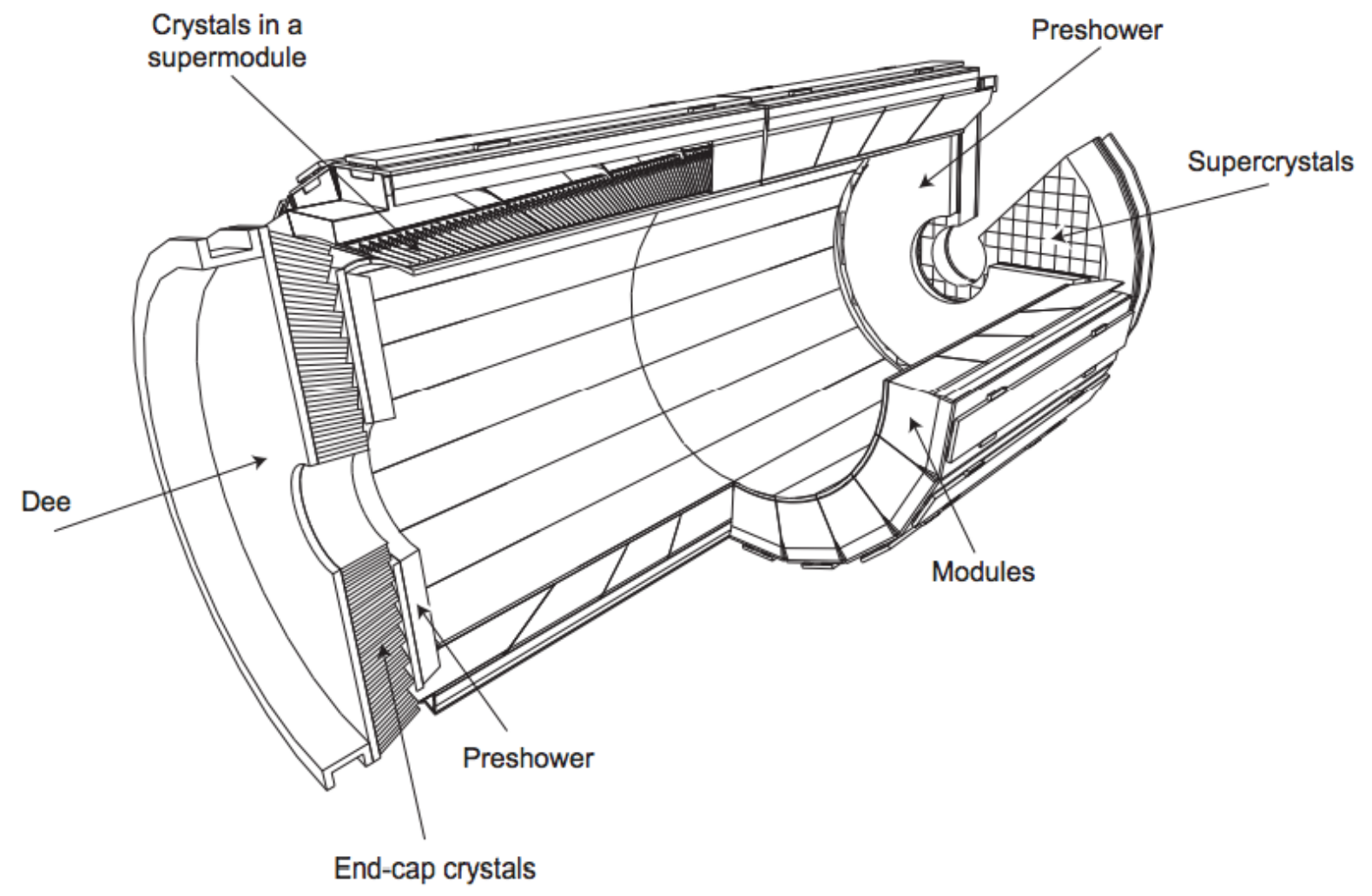

Figure 3.2.4: CMS electromagnetic calorimeter layout [25].

experiment. They are very dense $\left(8.28 \mathrm{~g} / \mathrm{cm}^{3}\right)$, have a short radiation length $(0.89 \mathrm{~cm})$, and have a small Molière radius $(2.2 \mathrm{~cm})$ [25]. This allows for a compact and high granularity calorimeter. Lead tungstate is optically transparent and emits blue-green scintillation when photons and electrons pass through, producing light in proportion to the particle's energy. $\mathrm{PbWO}_{4}$ crystals have a fast scintillation decay time: $\sim 80 \%$ of the light is emitted in the time between LHC bunch crossings, 25 ns. Although the light output is somewhat low and temperature dependent (at $18^{\circ} \mathrm{C}, \sim 4.5$ photoelectrons collected per $\mathrm{MeV}$ ), high-gain photodetectors can be used to read out the light signal. Additionally, the crystals used in CMS are required to be radiation hard.

The ECAL barrel is made up of 61,200 lead tungstate crystals and covers the pseudorapidity range of $|\eta|<1.479$. The front faces of the crystals are at $r=1.29 \mathrm{~m}$, and they are $230 \mathrm{~mm}$ long, corresponding to $25.8 X_{0}$. They are tapered, with front (rear) faces $\sim 22 \times 22$ $(26 \times 26) \mathrm{mm}^{2}$ in area. The crystals are arranged in a quasi-projective manner so that the 
cracks between crystals do not line up with particle trajectories. Groups of $2 \times 5$ crystals are arranged into submodules, each surrounded by a thin alveolar wall made of aluminum and glass fibre-epoxy resin. Forty to fifty submodules are grouped into modules, based on their position in $\eta$. Groups of four modules are housed in a supermodule, which contains 1700 crystals. In total, the EB crystals have a volume of $8.14 \mathrm{~m}^{3}$ and weigh $67.4 \mathrm{t}$.

Mounted to the back of each EB crystal is a pair of avalanche photodiodes (APDs) [25], read out in parallel. Through the photoelectric effect, APDs convert the light from the crystals into electrons and then provide signal gain through electron avalanche multiplication. Each APD has an active area of $5 \times 5 \mathrm{~mm}^{2}$. They are operated at a gain of 50 and a temperature of $18^{\circ} \mathrm{C}$. In order to reduce the nuclear effects of particles traversing the sensors, the APDs are thin $(6 \mu \mathrm{m})$. When a minimum ionizing particle (MIP) crosses an APD, it creates a signal equivalent to $100 \mathrm{MeV}$ deposited in the crystal. As with the crystals, the APDs are tested to ensure radiation tolerance.

Covering the pseudorapidity range $1.479<|\eta|<3.0$, the ECAL endcaps are located 315.4 $\mathrm{cm}$ from the IP, in the $z$-direction [25]. The EE crystals are grouped into supercrystals, which each contain $5 \times 5$ crystals and are surrounded by a carbon-fiber alveolar wall. Each endcap is divided into two halves, or Dees, which each hold 3,662 crystals. The crystals are arranged in an $\mathrm{x}^{-} \mathrm{y}$ grid and are angled between two and eight degrees from the $z$-axis. They have a front face cross section of $28.62 \times 28.62 \mathrm{~mm}^{2}$, a rear face cross section of $30 \times 30 \mathrm{~mm}^{2}$, and a length of $220 \mathrm{~mm}$, or $24.7 X_{0}$. The EE crystals make up a total volume of $2.90 \mathrm{~m}^{3}$ and weigh 24.0 t.

Due to the nonuniform magnetic field and high radiation doses in the endcaps, vacuum phototriodes (VPTs) were chosen to be used as the EE photodetectors. VPTs are photomultipliers with a single gain stage. The EE VPTs have a low quantum efficiency and a gain of only $\sim 10$, but this is offset by the large active area (about $280 \mathrm{~mm}^{2}$ ) [25]. One VPT is glued to the back of each EE crystal.

In between the ECAL barrel and endcap detectors lies a sampling calorimeter called 
the preshower detector [25]. It was designed to identify neutral pions in the endcap region between $1.653<|\eta|<2.6$, distinguish electrons from MIPs, and improve the position resolution of the photons and electrons identified in the ECAL. In both the $\pm z$ sides, the preshower consists of a 20-cm-thick disk. Each disk is made up of two layers, each with a lead layer for initiating electromagnetic showers, followed by a layer of silicon strip sensors used to measure the energy deposited and the transverse shower profiles. The strips in the two sensor planes are arranged perpendicularly to one another.

For energies below $500 \mathrm{GeV}$, the ECAL energy resolution is described by:

$$
\left(\frac{\sigma}{E}\right)^{2}=\left(\frac{S}{\sqrt{E}}\right)^{2}+\left(\frac{N}{E}\right)^{2}+C^{2}
$$

where $S$ is the stochastic term, $N$ is the noise term, and $C$ is the constant term. Three factors contribute to the stochastic term: fluctuations in the lateral shower containment, a $2.1 \%$ photostatistics contribution from the uncertainty in the number of primary photoelectrons released per $\mathrm{GeV}$, and fluctuations in the energy deposited in the the preshower absorber as compared to preshower silicon detector measurements. The noise term also has three contributions: electronics noise, digitization noise, and pileup noise. Finally, the constant term is primarily affected by intercalibration errors, nonuniformity of longitudinal light collection, and energy leakage from the back of the crystals [25].

\subsubsection{Hadronic Calorimeter}

The hadronic calorimeter (HCAL) makes up the third layer of CMS detectors. It is used to measure hadronic jets as well as missing transverse energy, indicative of neutrinos or exotic new particles [25]. The HCAL is split into four subsystems: the HCAL barrel (HB), endcap (HE), outer (HO), and forward (HF) detectors. Figure 3.2.5 shows an $r-z$ view of the CMS detector, indicating the positions of the HCAL subdetectors.

The HB is a sampling calorimeter which is situated radially between the ECAL (outer 


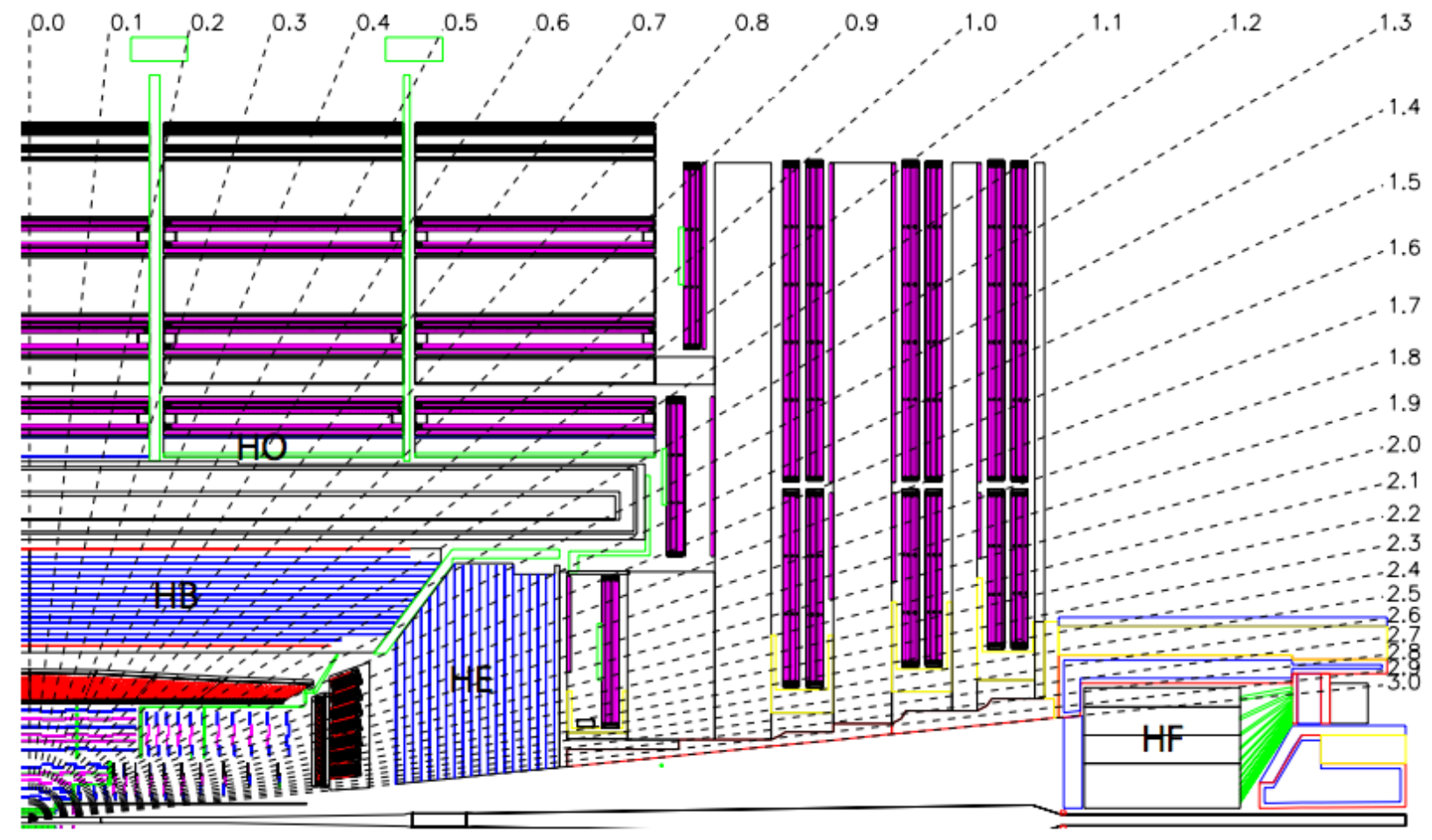

Figure 3.2.5: Cross section of the CMS hadronic calorimeter, indicating the positions of the hadron barrel (HB), endcap (HE), outer (HO) and forward (HF) calorimeters [25]. The dashed lines represent the $\eta$ coordinate. 
radius of $1.77 \mathrm{~m}$ ) and the magnet coil (inner radius of $2.95 \mathrm{~m}$ ). It covers the pseudorapidity range of $|\eta|<1.3$ [25]. The HB is made up of 36 azimuthal wedges, half of which form the HB + half-barrel and the other half of which make up HB-. Each wedge consists of alternating layers of absorber and plastic scintillator. There are sixteen layers of absorber plates, which are aligned parallel to the beam axis. They are bolted together in an overlapping manner so that there is no projective dead material. The innermost and outermost absorber layers are made out of 40-mm- and 75-mm-thick steel plates, respectively, which also provide structural support. The other fourteen absorber layers consist of brass plates, the first eight of which are $50.5 \mathrm{~mm}$ thick and the last six of which are $56.5 \mathrm{~mm}$ thick. The HB brass is $30 \%$ zinc and $70 \%$ copper, with a density of $8.53 \mathrm{~g} / \mathrm{cm}^{3}$ and an interaction length of $16.42 \mathrm{~cm}$.

There are seventeen layers of plastic scintillator in the HB [25]. Layer 0 lies in between the ECAL and the first steel HB plate. It is $9 \mathrm{~mm}$ thick and made of Bicron BC408. Its purpose is to sample the hadronic showers that develop in the material between the ECAL and HCAL. Each of the rest of the scintillator layers sit behind a layer of HB absorber. They are made of Kuraray SCSN81, a moderately radiation hard plastic scintillator. The brass-succeeding layers are $3.7 \mathrm{~mm}$ thick, while the final layer is $9 \mathrm{~mm}$ thick. The greater thickness of the last layer of scintillator is used to correct for the late developing showers that leak out of the HB. The scintillator is segmented in $\eta$ and $\phi$, resulting in a granularity of $(\Delta \eta, \Delta \phi)=(0.087,0.087)$. A single unit of scintillator is known as a tile; the CMS HCAL contains about 70,000 tiles.

When a hadron passes through the HB, it interacts strongly with the nuclei of the absorber material, creating a shower of quarks and gluons. These particles deposit their energy in the scintillator layers, causing them to produce a blue-violet light. Wavelength-shifting (WLS) fibres, $0.94 \mathrm{~mm}$ in diameter, collect this light, shift it toward the green end of the spectrum, and carry it to hybrid photodiodes (HPDs) [25]. The HPDs read out the light and provide a gain of approximately 2000. Multipixel HPDs were chosen to be used as the HCAL photodectors because of their ability to operate in high axial magnetic fields and to 
proportionally amplify signals over a large range of particle energies.

Because the HB is radially restricted by the ECAL and magnet, a complementary outer barrel calorimeter $(\mathrm{HO})$ is placed outside the magnet volume to catch the tails of the hadronic showers [25]. The HO uses the solenoidal coil as the absorber material. Outside of the solenoid, the magnetic field is returned through an iron yoke made of five rings, which encircle the ECAL and are $2.536 \mathrm{~m}$ wide in the $z$-direction. The HO makes up the first sensitive layer in each of these rings. The depth of the HB absorber is at a minimum at $\eta=0$. Therefore, the central ring of HO (ring 0) has two layers of 10-mm-thick scintillator, which lie at $r=3.82 \mathrm{~m}$ and $r=4.07 \mathrm{~m}$, on either side of a 19.5-cm-thick layer of iron. Rings \pm 1 and \pm 2 each contain a single layer of scintillator, placed at $r=4.07 \mathrm{~m}$. Each ring is divided into $12 \phi$-sectors, which are separated by 75 -mm-thick stainless steel beams that are used to hold the magnet return yoke and the barrel muon system. HO tiles are designed to achieve the same $\eta-\phi$ granularity and utilize the same light collection method as in the HB. Studies of physics simulation have shown that the inclusion of the HO decreases the leakage of energy from the CMS calorimeter system and improves the measurement of missing energy.

As with the other CMS detector subsystems, the HCAL has an endcap component, the HE. It covers the pseudorapidity range $1.3<|\eta|<3$ and consists of two disks (one in either direction in $z$ ) attached to the muon endcap yokes [25]. Each disk comprises 36 wedges in $\phi$ and 18 layers of absorber and scintillator. The absorber layers are made from 79-mmthick C26000 cartridge brass, which is nonmagnetic, has high radiation tolerance, and is inexpensive. The plates are arranged so as to minimize cracks between the HB and HE and to eliminate dead material. The plastic scintillator layers are $3.7 \mathrm{~mm}$ thick (layers $1-17$ ), except for the 9-mm-thick layer 0 . In the HE, the scintillator tiles are trapezoidal in shape and provide a granularity of $\Delta \eta \times \Delta \phi=0.087 \times 0.087(\Delta \eta \times \Delta \phi \approx 0.17 \times 0.17)$ for $|\eta|<1.6$ $(|\eta| \geq 1.6)$. As with the HB and HO, the light signal is read out using WLS fibres and HPDs.

Finally, the HF covers the most forward region $(3.0<|\eta|<5.0)$ of the HCAL. Each side 
of the $\mathrm{HF}$ is a cylindrical steel structure with an inner radius of $12.5 \mathrm{~cm}$, an outer radius of $130.0 \mathrm{~cm}$, and a front face $11.2 \mathrm{~m}$ from the IP [25]. Each structure is made up of 5-mmthick, 165-cm-long (in z) steel plates, which are grouped into 18 azimuthal wedges. Due to extremely high particle fluxes ( $~ 760 \mathrm{GeV}$ is deposited in HF per proton-proton interaction, vs $100 \mathrm{GeV}$ in the rest of the HCAL), quartz fibres are used as the active medium. Charged particles with energy above the Cherenkov threshold ( $\mathrm{E} \geq 190 \mathrm{keV}$ ) produce Cherenkov light in the fibres, which is then read out using photomultiplier tubes (PMTs). The fibres are inserted into grooves in the steel plates, which run parallel to the beam line. They are then bundled to form $\Delta \eta \times \Delta \phi=0.175 \times 0.175$ towers. Electrons and photons deposit most of their energy in the first $22 \mathrm{~cm}$ of the detector, while hadron showers produce nearly equal signals before and after this $22 \mathrm{~cm}$ mark. Therefore, in order to distinguish electronic and hadronic showers, half of the HF fibres run over the full depth of the absorber $(165 \mathrm{~cm})$, while the other half start at a depth of $22 \mathrm{~cm}$. Long and short fibres alternate in adjacent grooves, and the two sets are read out separately.

The HF is also used to measure the instantaneous luminosity of the LHC [25]. One method employed by CMS is counting the average fraction of empty towers, which indicates the average number of interactions per bunch crossing. The second method profits from the linear relationship between the luminosity and the average transverse energy per tower.

The thickness of the HCAL, in nuclear interaction lengths, varies from 10-15 $\lambda_{I}$, depending on $\eta$ [25]. At $\eta=0$, the total absorber thickness of the HB is $5.82 \lambda_{I}$. The effective thickness increases with polar angle as $1 / \sin \theta$; therefore the thickness of the HB is $10.6 \lambda_{I}$ at $|\eta=1.3|$. In front of the HB, the ECAL adds $\sim 1.1 \lambda_{I}$ of material. Additionally, the HO uses the solenoidal coil to add $1.4 / \sin \theta$ interaction lengths, extending the depth of the calorimeter system to a minimum of $11.8 \lambda_{I}$. The length of the calorimeter, including both HCAL and ECAL, is about $10 \lambda_{I}$. Figure 3.2.6 shows the thickness of CMS in interaction lengths as a function of $\eta$. 


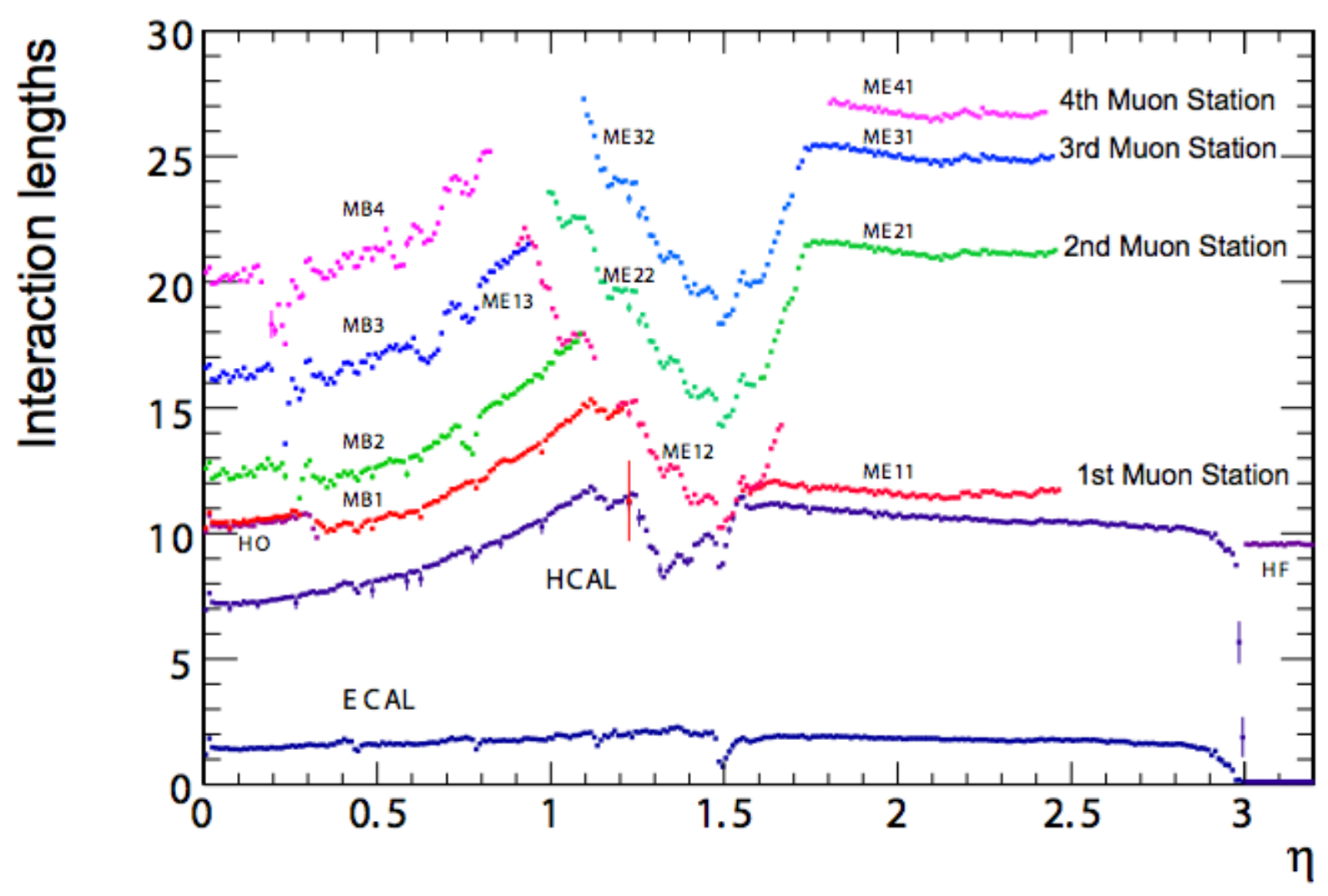

Figure 3.2.6: Thickness of the CMS detector material, in nuclear interaction lengths $\lambda_{I}$, as a function of pseudorapidity [25]. 


\subsubsection{Magnet}

Surrounding the tracker, ECAL, and HCAL, is the superconducting solenoid magnet. It is $6 \mathrm{~m}$ in diameter, $12.5 \mathrm{~m}$ in length, and weighs $220 \mathrm{t}$. At full current, $19.14 \mathrm{kA}$, the magnet achieves a $3.8 \mathrm{~T}$ internal magnetic field and stores 2.6 GJ of energy. In order to generate such a large field, the solenoid winding is composed of four layers, more than the usual one or two. The winding is made up of a NbTi conductor reinforced with aluminum [25]. This structural reinforcement is necessitated by the large mechanical deformation $(0.15 \%)$ that occurs when the magnet is turned on, caused by the large amount of energy stored per unit of the cold mass $(11.6 \mathrm{KJ} / \mathrm{kg})$. The cold mass and a cross sectional view of the four-layer winding can be seen in Fig. 3.2.7.

The magnet flux is returned in a 10,000 $\mathrm{t}$ iron yoke, comprising six endcap disks (three on each end) and five barrel wheels. The muon detectors are housed between the layers of this return yoke. While the magnetic field is quite uniform within the solenoidal volume and the return flux is mostly contained within the yoke, there is some nonzero magnetic flux within muon system volume. This most greatly affects the endcap muon systems closest to the IP, where the magnetic field is large and nonuniform.

\subsubsection{Muon System}

Muons experience less radiative loss in the tracker than kinematically similar electrons and are also expected to be the only directly detectable particles that survives past the calorimeter. Therefore, the muon system makes up CMS's outermost detector subsystem. Layered within the magnet's iron flux return yoke, it provides muon identification, momentum measurement, and triggering. The geometry of the high-field magnet and its flux-return yoke provides strong muon bending power and confines a large portion of the backgrounds to the endcap muon detectors closest to the IP, thus enabling good momentum resolution and triggering. The flux-return yoke also acts as a hadron absorber for muon ID [25].

CMS's muon system was designed to provide good reconstruction of muon momentum 

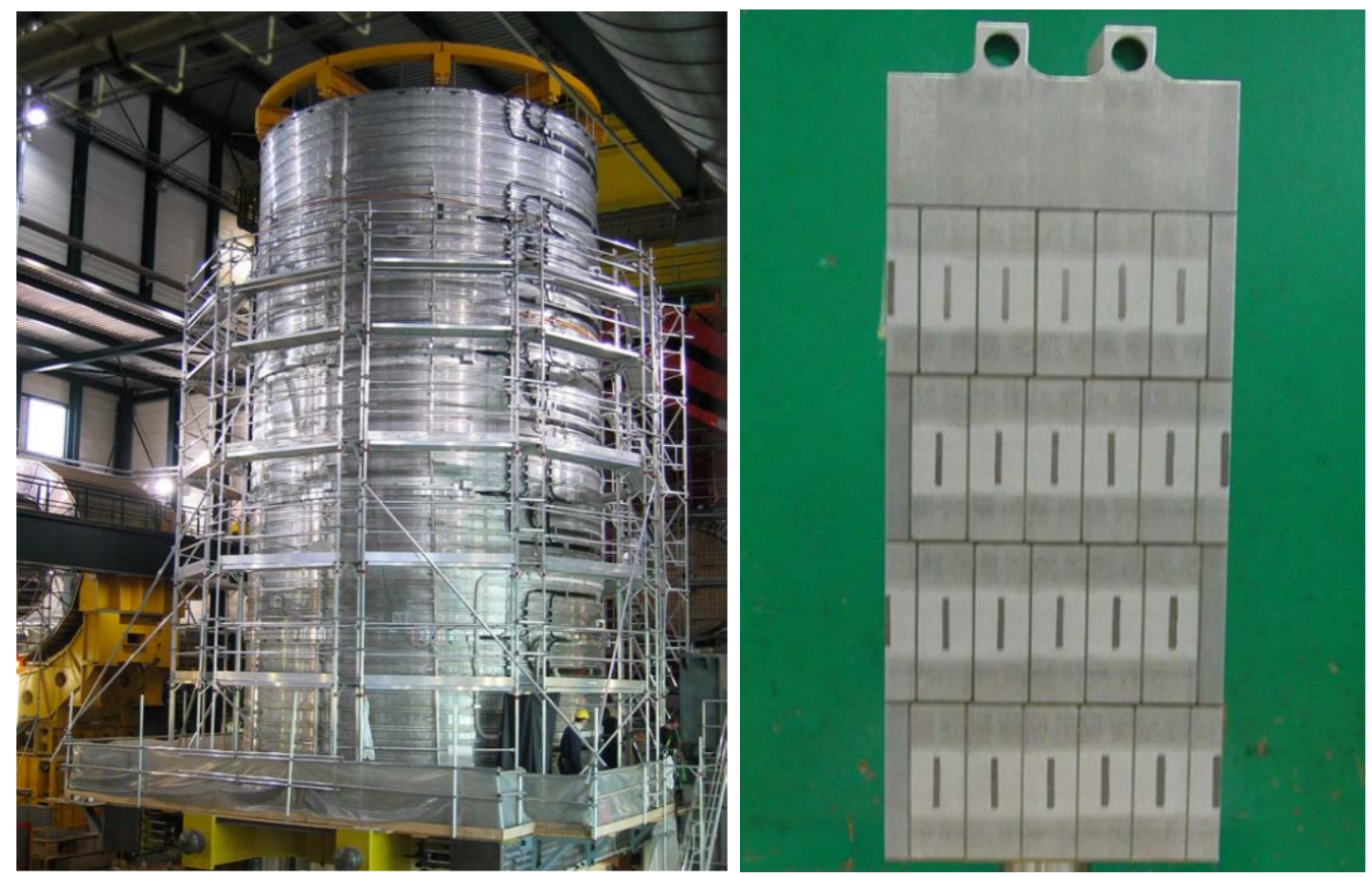

Figure 3.2.7: The cold mass of the CMS magnet. The entire volume is shown on the left, while a cross-sectional view of the four-layer winding is shown on the right [25].

and charge over the whole kinematic range of the LHC. Due to the arrangement of the magnet and flux-return yoke, the muon system comprises a cylindrical barrel region and two endcap disk sections, similar to the other detector subsystems. In order to instrument 25,000 $\mathrm{m}^{2}$ of muon detection planes, the muon chambers were designed to be inexpensive, reliable, and robust. Muon drift tube (DT) chambers are located in the barrel region, cathode strip chambers (CSC) are located in the endcap region, and resistive plate chambers (RPC) are located in both the barrel and encap regions. All three types of muon detector are gaseous. Fig. 3.2.8 shows a quadrant of the CMS muon detector, with the DTs, CSCs, and RPCs depicted in tan, green, and blue, respectively. The muon detectors to be installed for the HL-LHC are shown in red and purple, including a new type of muon detector, the gas electron multiplier (GEM) chambers. My work on the CSC and GEM detectors is described in Appendix A. 


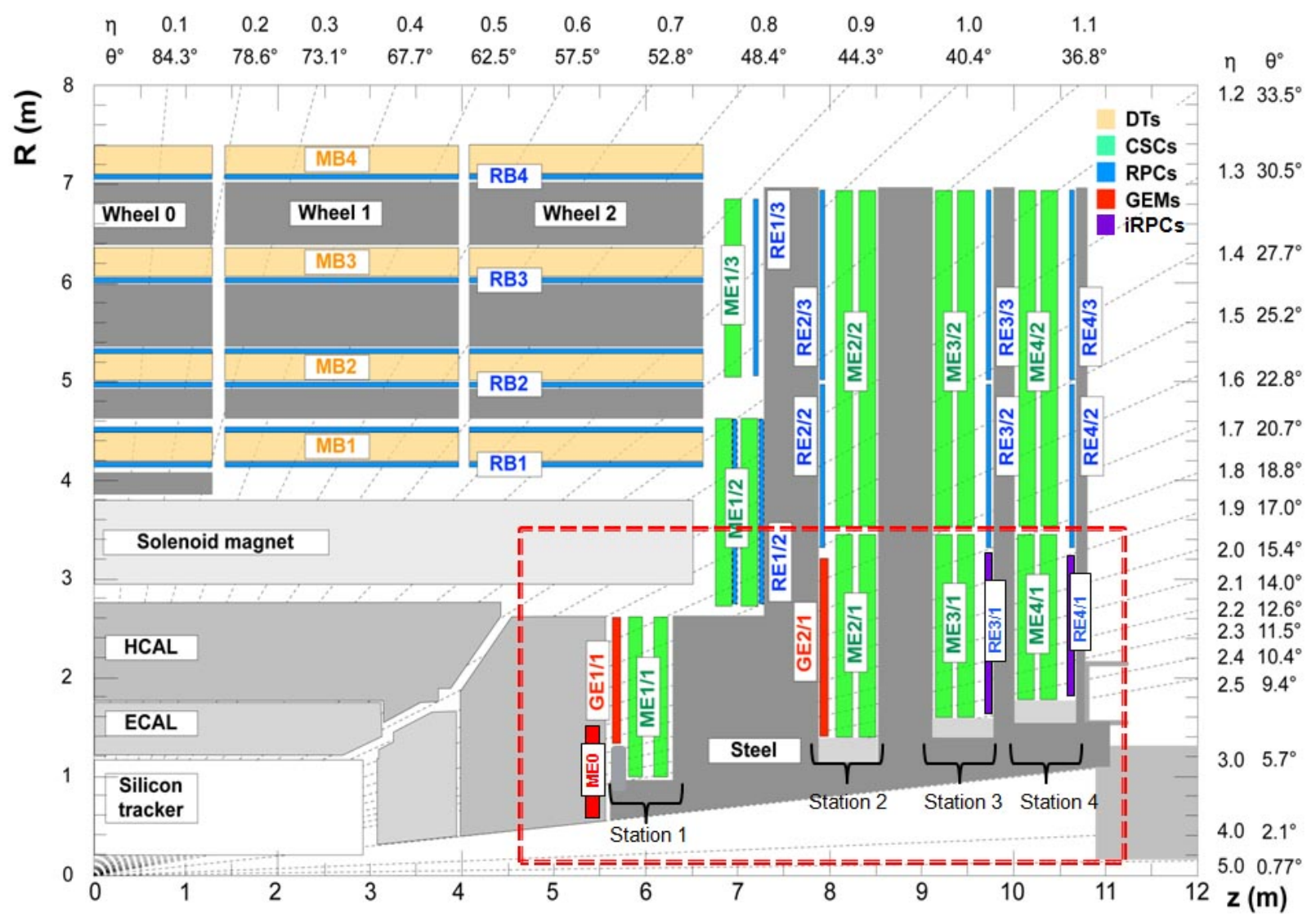

Figure 3.2.8: A quadrant of the CMS detector. The current muon detectors are shown in tan, green, and blue, while the proposed Phase-II upgrade muon detectors are shown in red (GEMs) and purple (iRPCs) [28]. 


\section{Drift Tubes}

In the barrel region, the muon rate is low, the neutron-induced background is small, and the 4-T magnetic field is uniform and mostly contained in the iron flux-return yoke. Therefore, drift tube chambers, consisting of rectangular drift tube cells, are used for muon identification and tracking [25]. The CMS barrel muon detector is made up of four stations, which form concentric cylinders around the beam line. The outermost cylinder contains 70 DT chambers, while the other three have 60 each. The DT system covers the pseudorapidy region $|\eta|<1.2$. The layout of the CMS muon drift tubes in one of the five barrel wheels is shown in Fig. 3.2.9.

A DT chamber is made up of drift cells arranged into Superlayers (SL). A single drift cell has a cross section of $13 \times 42 \mathrm{~mm}^{2}$, centered on a 2.4 -m-long anode wire contained within a gas volume ( $85 \% \mathrm{Ar}+15 \% \mathrm{CO} 2)$, shown in Fig. 3.2.10. A charged particle passing through a drift cell will ionize the gas, causing a cascade of charged particles, which are collected by the anode wire and cathode strip. The signal is then amplified by the readout system.

One SL is made of 4 consecutive layers of cells, staggered by half a cell. A DT chamber is then made of two or three SLs. The wires in the outer two SLs are parallel to the beam line, providing a muon track measurement in $r-\phi$. The wires in the innermost SL are perpendicular to the beam line, proving a track measurement in the $z$-direction. The chambers in the fourth DT station do not contain this third SL layer and therefore do not provide a measurement in $z$. The chambers were designed to have a global resolution of $100 \mu \mathrm{m}$ in $r-\phi$. The design of the overall DT system allows for a barrel muon momentum measurement that provides $>95 \%$ efficiency for reconstructing a high- $p_{T}$ muon in the pseudorapidity range covered by four stations.

\section{Cathode Strip Chambers}

In the endcap region, the muon rates and background levels are high, while the magnetic field is large and nonuniform. CMS therefore employs CSCs - which are radiation resistant, finely segmented, and have a fast response time - for endcap muon detection [25]. The CSC system 


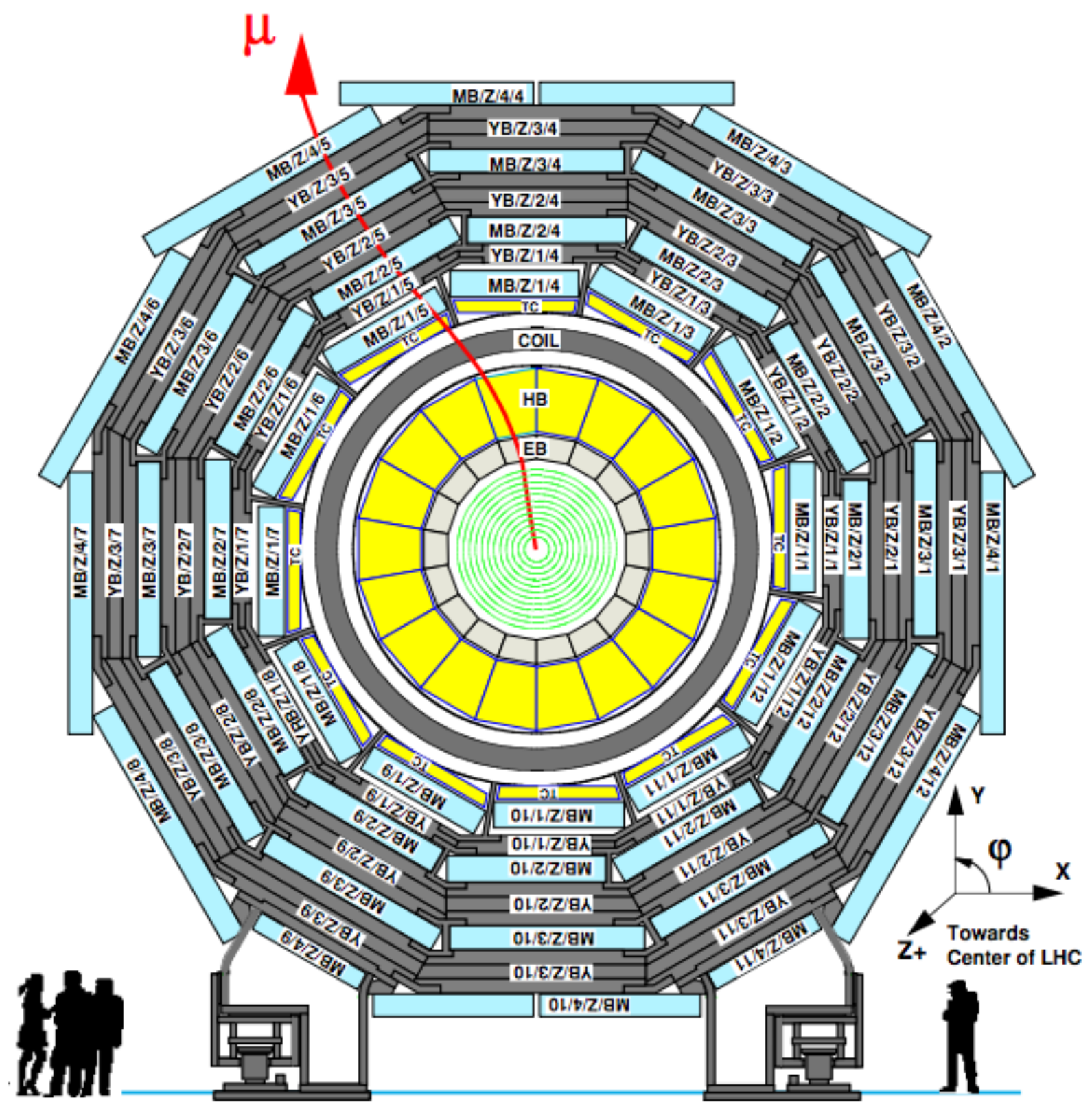

Figure 3.2.9: Layout of the CMS muon drift tubes (light blue) in one of the five wheels [25]. 


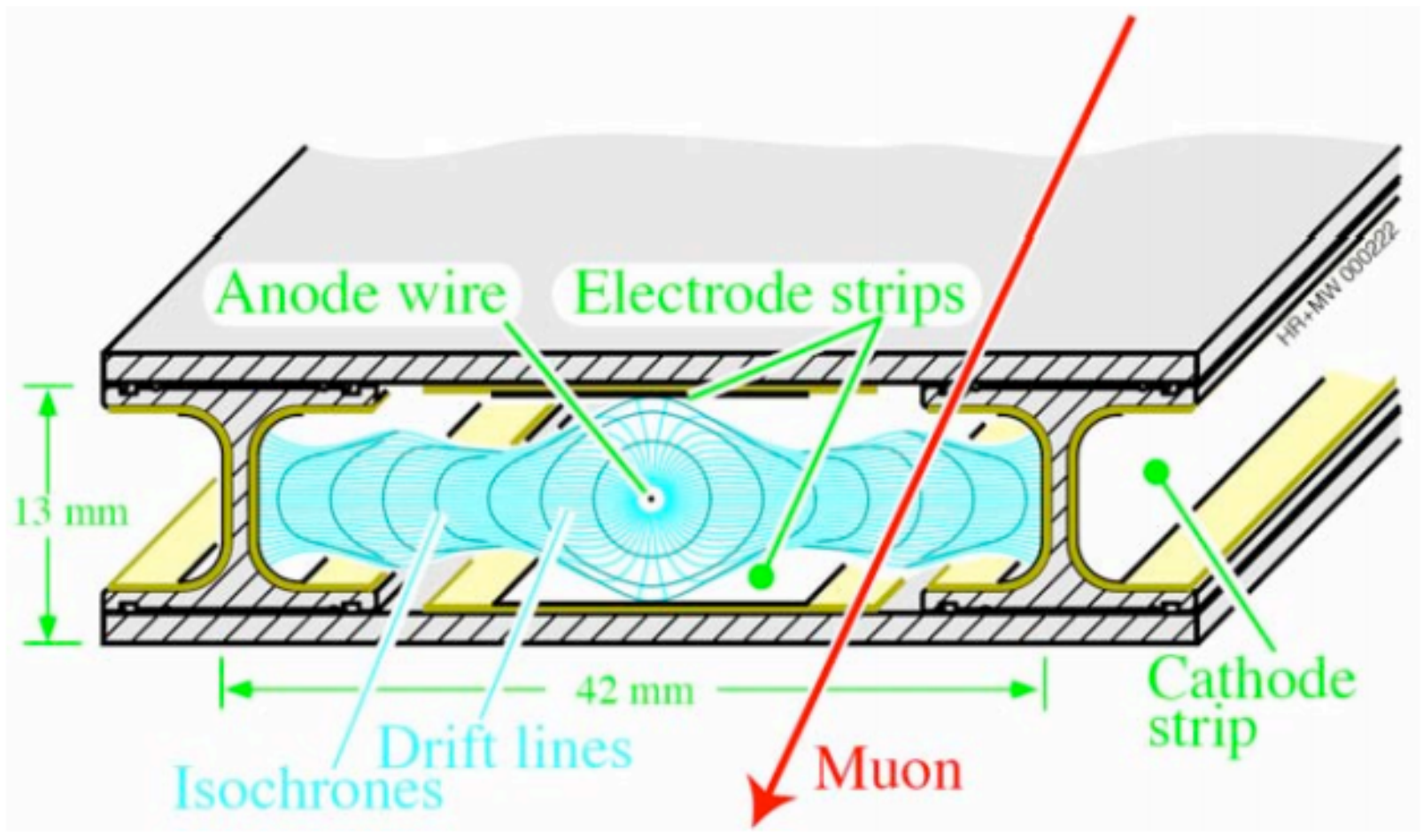

Figure 3.2.10: Cross section of one of the CMS muon drift tubes [25].

consists of 540 trapezoidal cathode strip chambers, arranged in four disks, or stations, on each end of CMS $( \pm z)$. Specifically, there are 72 ME1/1 chambers, 72 ME1/2, 72 ME1/3, $36 \mathrm{ME} 2 / 1,72 \mathrm{ME} 2 / 2,36 \mathrm{ME} 3 / 1,72 \mathrm{ME} 3 / 2,36 \mathrm{ME} 4 / 1$, and $72 \mathrm{ME} 4 / 2$ chambers. An individual chamber is referred to as, e.g. ME $+1 / 2 / 15$, where ME stands for "muon endcap," and the chamber is in the 15 th position, on the 2 nd innermost ring (as measured from $r=0$ ), on the first disk from $z=0$, on the $+z$ endcap. Figure 3.2.11 shows an ME2 ring.

Each CSC chamber covers either $10^{\circ}$ or $20^{\circ}$ in $\phi$, and except for the ME1/3 ring, all chambers overlap, providing contiguous coverage in $\phi$. Muons traversing the detector between $1.2<|\eta|<2.4$ will cross three or four CSCs. Between $0.9<|\eta|<1.2$, the barrel and endcap muon detectors overlap, and muons are therefore detected by both CSCs and DTs. Muons in the pseudorapidity range $|\eta|<1.6$ are also detected by RPCs.

A single CSC chamber consists of six anode wire planes alternately layered between seven cathode strip panels, within a gas volume (Fig. 3.2.12, left). The wires run along 


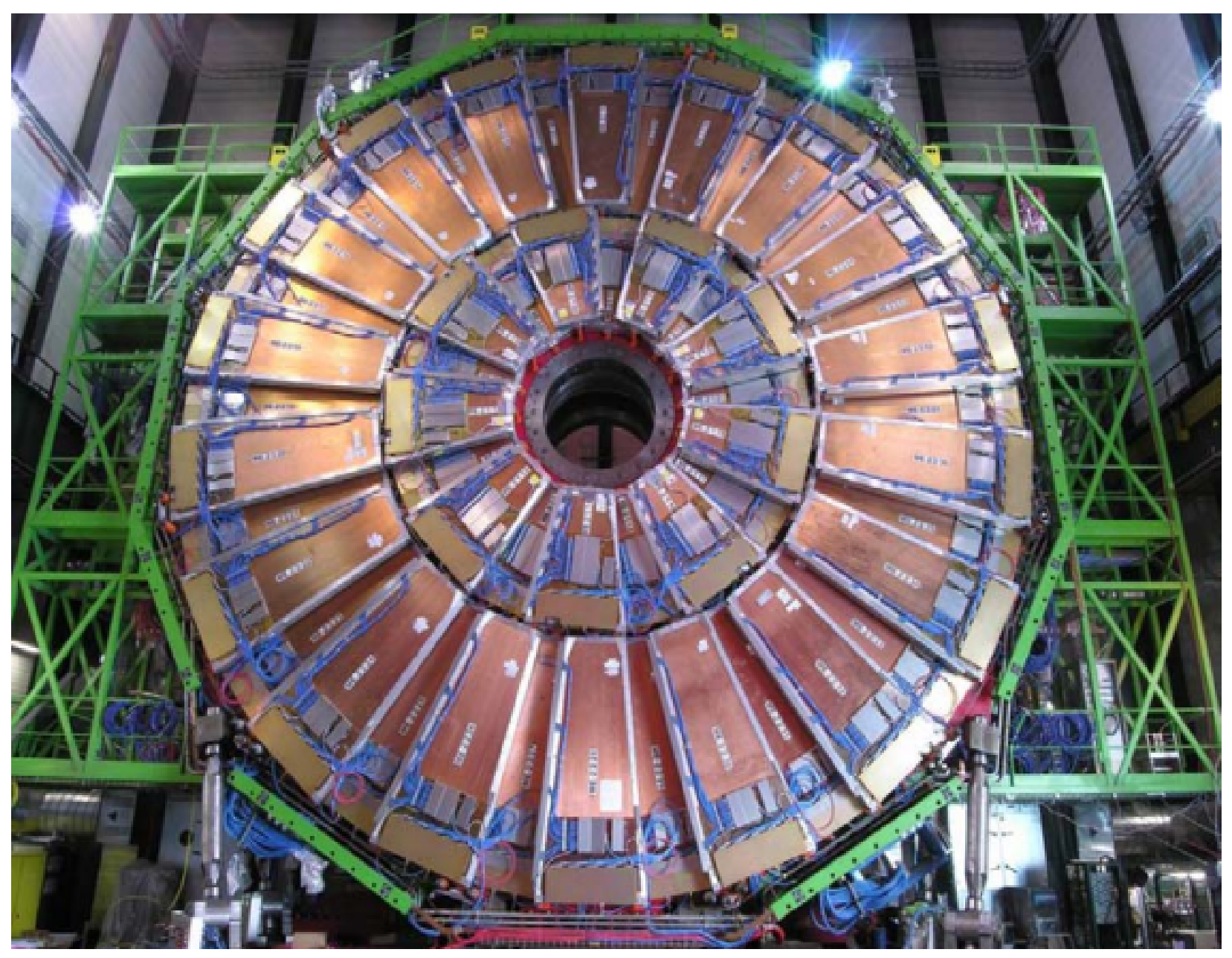

Figure 3.2.11: The ME2 wheel of the CMS muon cathode strip chambers. The overlap of the chambers provides continuous coverage in the azimuthal angle $\phi[25]$. 

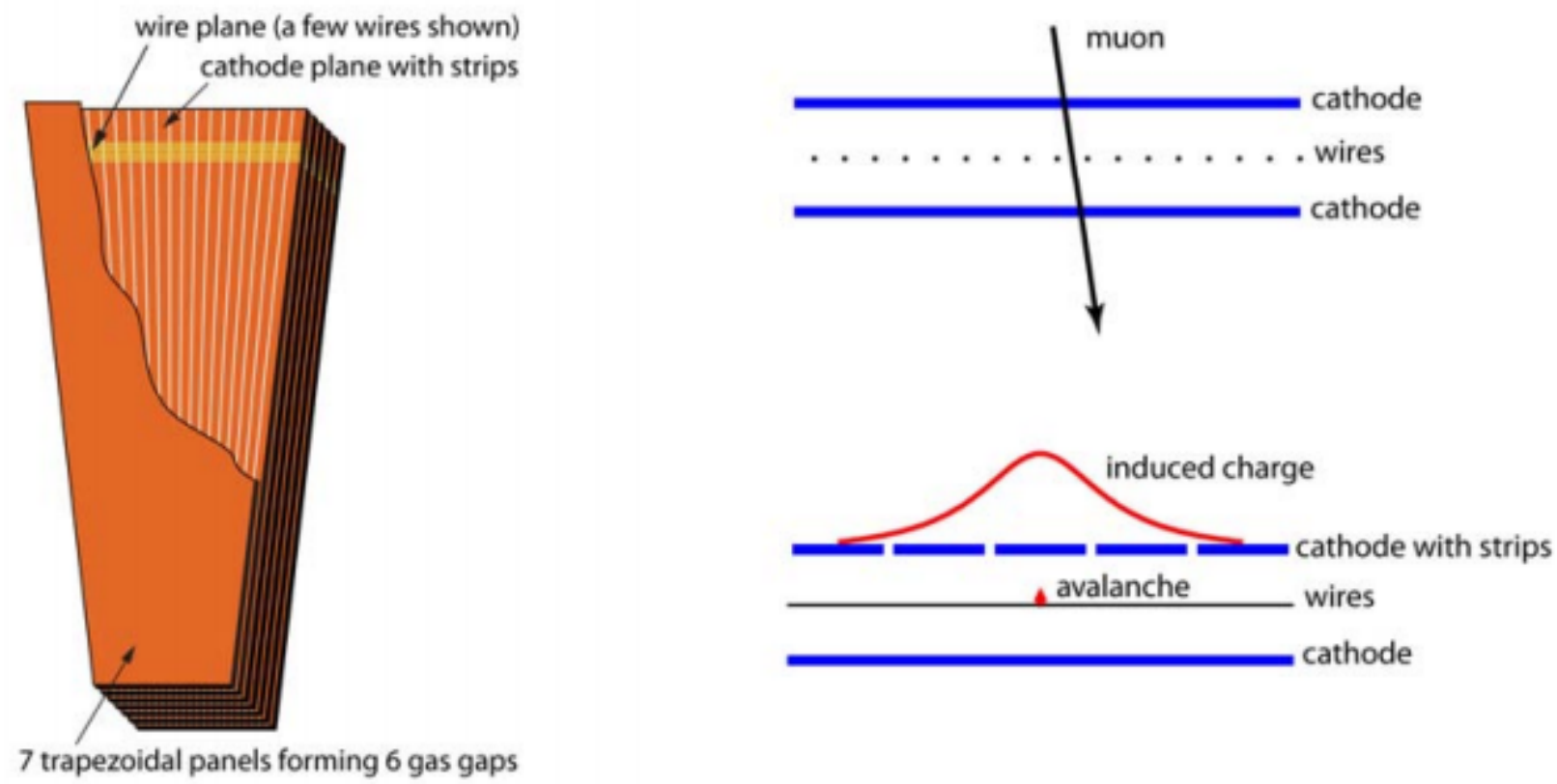

Figure 3.2.12: (Left) Layout of a CMS cathode strip chamber, revealing the anode wires and cathode strips in the top layer. (Right) An illustration of muon detection in one CSC layer. A muon ionizes the gas, inducing a charge on the nearest wires and strips [25].

the $\phi$-direction, measuring a muon's radial position. The cathode panel strips run along the $r$-direction and have a constant azimuthal width. The CSCs are gas ionization chambers, operating under the same principles as the DTs (Fig. 3.2.12, right). They contain a $40 \% / 50 \% / 10 \%$ gas mixure of $\mathrm{Ar} / \mathrm{CO}_{2} / \mathrm{CF}_{4}$, where the $\mathrm{CO}_{2}$ is a nonflammable quencher that enables large gas gains and the $\mathrm{CF}_{4}$ helps to prevent wire polymerization.

The ME1/1 chambers, which reside within the solenoidal volume and experience the uniform $4 \mathrm{~T}$ magnetic field, have a specialized design. In order to maintain performance under higher background rates, the ME1/1 chambers are split into two sectors in $|\eta|$. Additionally, the anode wires are tilted by a $29^{\circ}$ angle, in order to compensate for the Lorentz angle of the charged particles induced in the gas volume. Overall, the sensitive chamber area is about $5000 \mathrm{~m}^{2}$, the volume of gas contained within the CSC system is greater than $50 \mathrm{~m}^{3}$, and there are about 2 million wires.

The CSCs both provide precise measurements of muon momenta and serve as a muon 
trigger. Each CSC chamber employs robust pattern recognition in order to reject non-muon backgrounds and efficiently match hits to those in other CSC stations and the inner tracker. A single chamber is about $99 \%$ efficient at finding level one trigger (L1T) track stubs and has at least a $92 \%$ probability of identifying the correct bunch crossing by the time the L1T is read out. A muon crossing three to four CSCs is therefore at least $99 \%$ likely to be assigned the correct bunch crossing number. The CSCs have about $2 \mathrm{~mm} r-\phi$ resolution at L1T, and 75 (150) $\mu \mathrm{m}$ offline $r-\phi$ resolution, in the case of (non)ME1/1 and ME1/2 chambers.

Considering both the DTs and CSCs, the standalone muon system's offline muon momentum resolution is $\sim 9 \%$ for low $\eta$ and $p$ muons with $p_{T}<200 \mathrm{GeV}$. This resolution is limited primarily by multiple scattering in the material in front of the first muon station. Depending on $|\eta|$, the standalone muon momentum resolution for a $1 \mathrm{TeV}$ muon is $15-40 \%$. At low momenta, including the inner tracker improves the resolution by an order of magnitude. At high momenta, around $1 \mathrm{TeV}$, a global momentum fit only yields a $\sim 5 \%$ resolution.

\section{Resistive Plate Chambers}

Due to uncertainty in the ultimate muon background rates and in the CMS muon system's ability to correctly measure the beam-crossing time during LHC operations at full luminosity, CMS added a complementary dedicated trigger system to both the barrel and endcap muon regions [25]. These resistive plate chambers are gaseous parallel-plate detectors, which are able to determine the time of an ionizing event with better resolution than the 25 ns between LHC collisions. They provide a fast and independent trigger with a sharp $p_{T}$ threshold and a coarse, but adequate, spatial resolution over the range $|\eta|<1$.6. They are double-gap chambers, which are operated in avalanche mode, ensuring good performance at the high rates expected at the LHC. A single RPC is shown in Fig. 3.2.13.

In the barrel region, the RPCs are arranged into 12 sectors around the DT layers in each of the five wheels, as shown in Fig. 3.2.14. In the two innermost stations, or rings, each DT layer has an RPC in front of it (RB1in and RB2in) and behind it (RB1out and RB2out), 


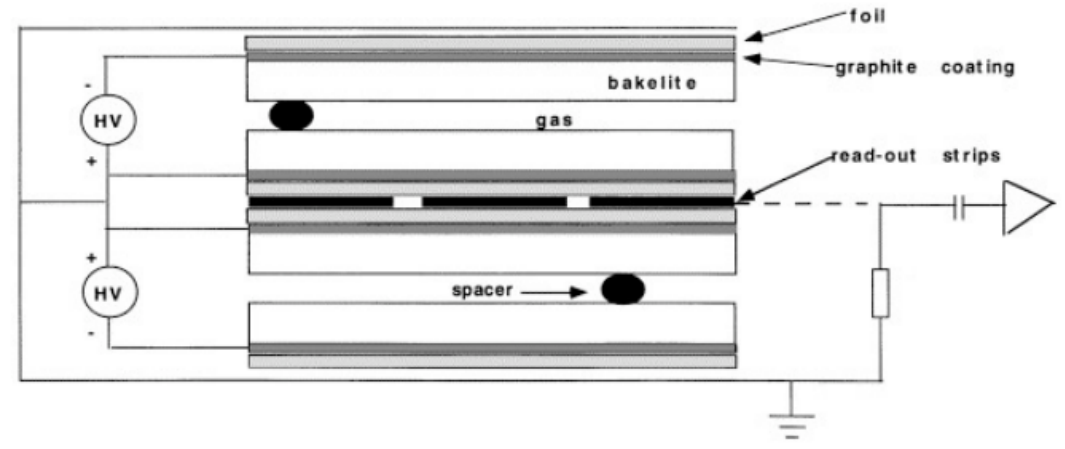

Figure 3.2.13: Cross section of one of the CMS muon resistive plate chambers [29].

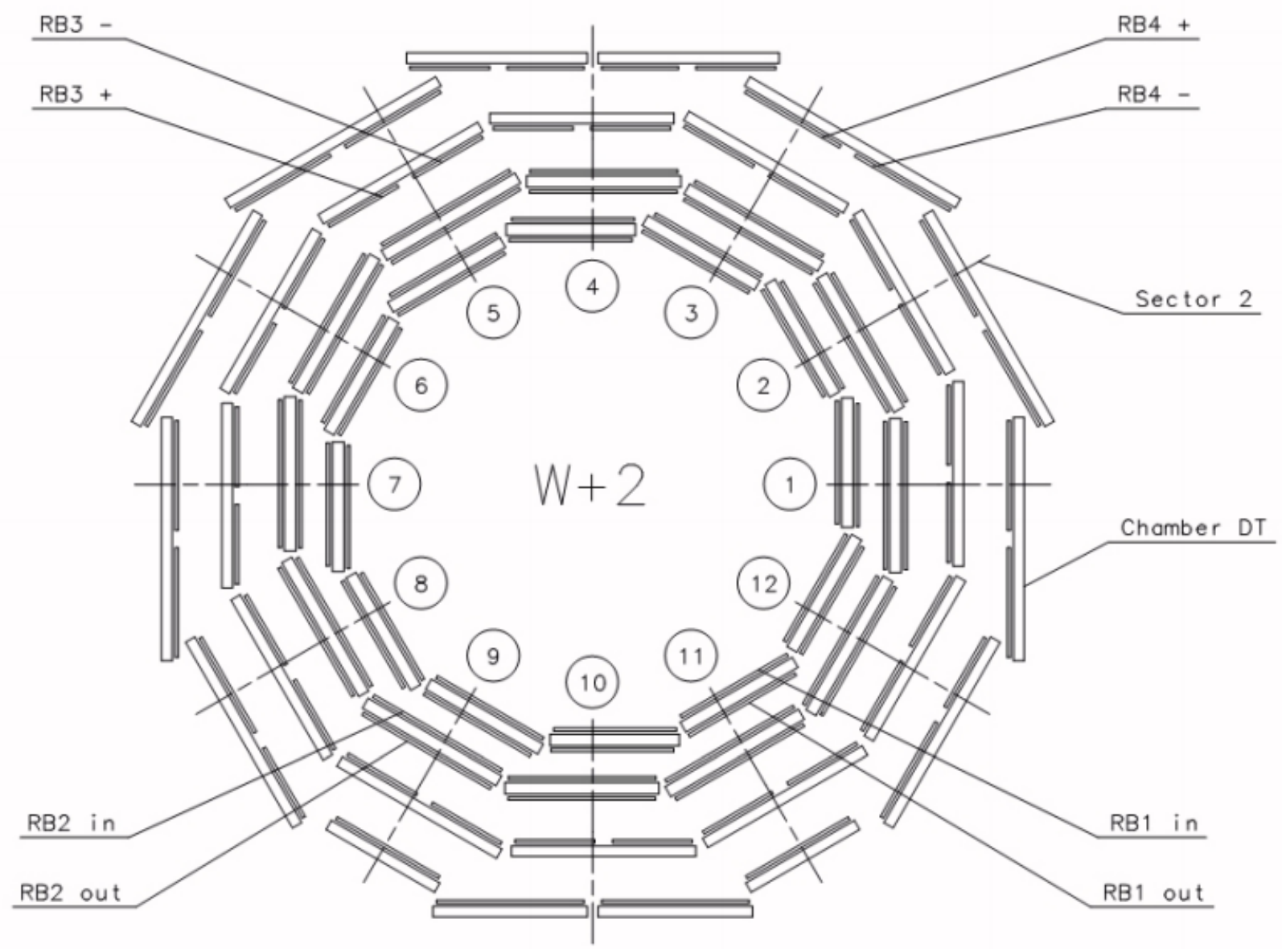

Figure 3.2.14: Layout of the CMS muon resistive plate chambers in one of the five wheels [25]. 
with respect to the $r$ coordinate. In the third and fourth stations, both RPCs are located on the inner side of the DT layer, denoted as RB3+/- and RB4+/-. The fourth station contains a few exceptions: sector 4 has 4 chambers (RB4++, RB4+, RB4-, RB4--), while sectors 9 and 11 each have one RPC. There are 480 rectangular barrel chambers, almost all of which are $2455 \mathrm{~mm}$ long in the $z$ direction. Each chamber consists of two or three double-gap modules arranged sequentially in the $z$ direction. Each double-gap module has up to 96 readout strips running along the beam direction. Each strip covers $5 / 16^{\circ}$ in $\phi$.

On each of the endcaps, the RPC stations are mounted in concentric rings on the disks, as shown in Fig. 3.2.8, Each double-gap chamber is trapezoidal in shape. Except in the case of station one, the chambers in the innermost ring cover $20^{\circ}$ in $\phi$, while the others cover $10^{\circ}$.

\section{Gas Electron Multipliers}

Studies of muon trigger rates have concluded that maintaining feasible L1 trigger (Sec. 3.2.7) rates for low $p_{T}(<25 \mathrm{GeV})$ muons after the 2019-2020 LS2 is not possible without incurring large losses in endcap muon trigger efficiencies [31]. Unfortunately, this would affect over half of CMS muon coverage. To address this, CMS plans to upgrade the muon system in order to maintain Run I muon performance in the high rate environment of the HL-LHC. One part of the muon upgrade is the installation of GEM chambers in front of the ME1/1 and ME2/1 chambers, as shown in Fig. 3.2.8.

First, pairs of GE1/1 chambers will be installed in front of the ME1/1 chambers, benefiting muon triggering and reconstruction in the $1.6<|\eta|<2.2$ region. Due to the large magnetic field at the first muon station, muon bending is larger than at the other endcap stations. The first muon station also receives the highest background rate and is therefore a large contributor to the trigger rate. At this location, the combination of GE1/1 and ME1/1 chambers will effectively multiply the muon path length by a factor of 2.4-3.5, as compared to the six ME1/1 layers alone. Because muon momentum resolution increases with path length, the addition of GE1/1s improves the accuracy of muon momentum measurements 


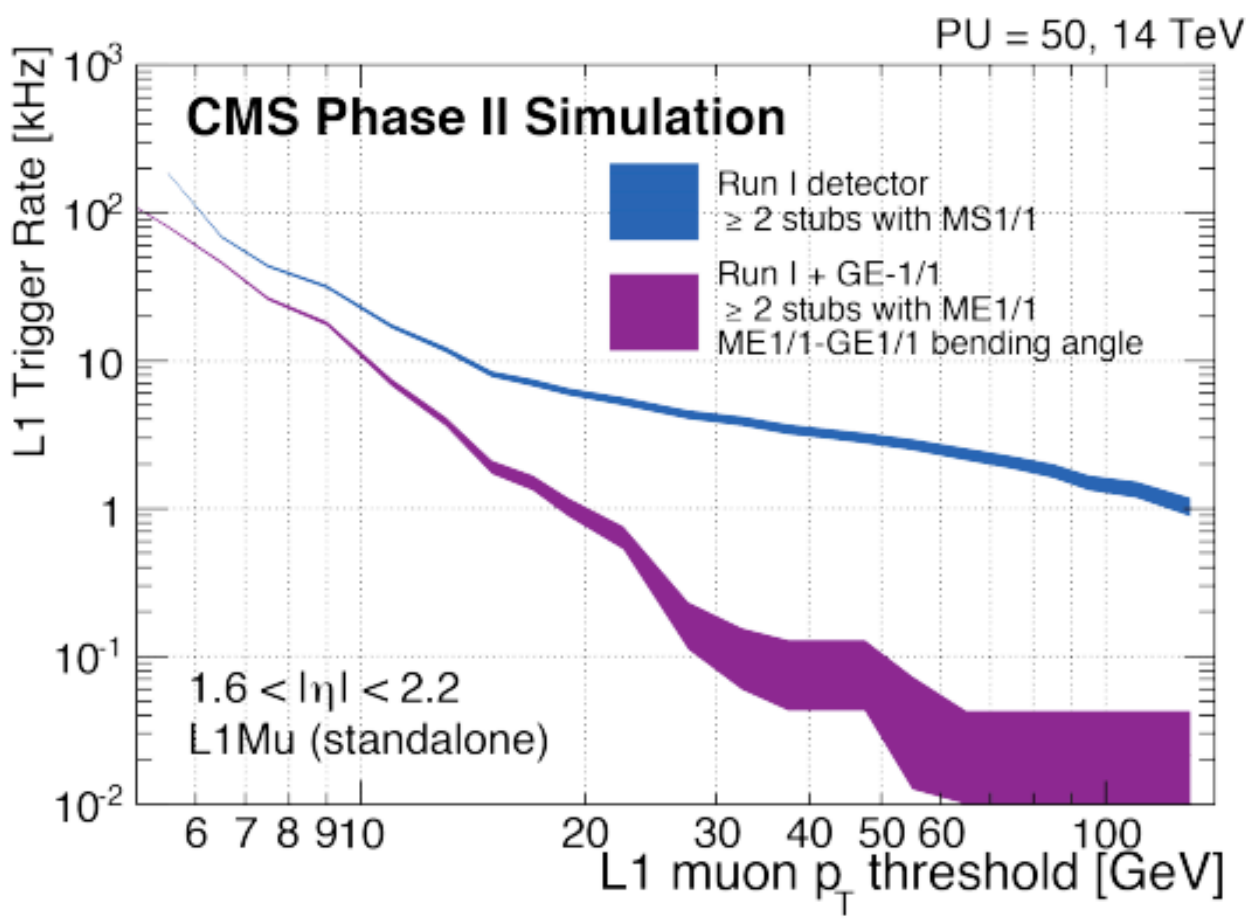

Figure 3.2.15: Level 1 muon trigger rates before (blue) and after the GE1/1 (magenta) upgrade. Adding the GE1/1 chambers greatly reduces the trigger rate [31].

and significantly reduces the large contribution to the L1 muon trigger rate. Figure 3.2.15 compares the single muon trigger rates in the region $1.6<|\eta|<2.2$ before and after the GE1/1 upgrade. With the inclusion of GEM chambers in the first endcap muon station, the L1 muon thresholds can be kept at low $p_{T}$ values, thereby maintaining high efficiency for recording physics processes that feature soft leptons. For example, the GE1/1 upgrade will enable the L1 single muon trigger threshold to stay at $12-14 \mathrm{GeV}$, with approximately full offline efficiency for $p_{T}>18-20 \mathrm{GeV}$ muons.

GEM chambers were chosen for this part of the endcap muon upgrade because they are capable of operating well at high HL-LHC rates. Additionally, they are small enough to fit in the spaces originally intended for additional RPCs (deemed unsuitable for the high particle flux). Figure 3.2.16 gives a side view of a single GEM chamber. As a muon passes through, it ionizes the gas $\left(\mathrm{Ar} / \mathrm{CO}_{2}\right.$ 70:30). Inside the gas volume, are three GEM foil layers (hence the name "Triple-GEM detector"), across which are applied a voltage difference on the order 


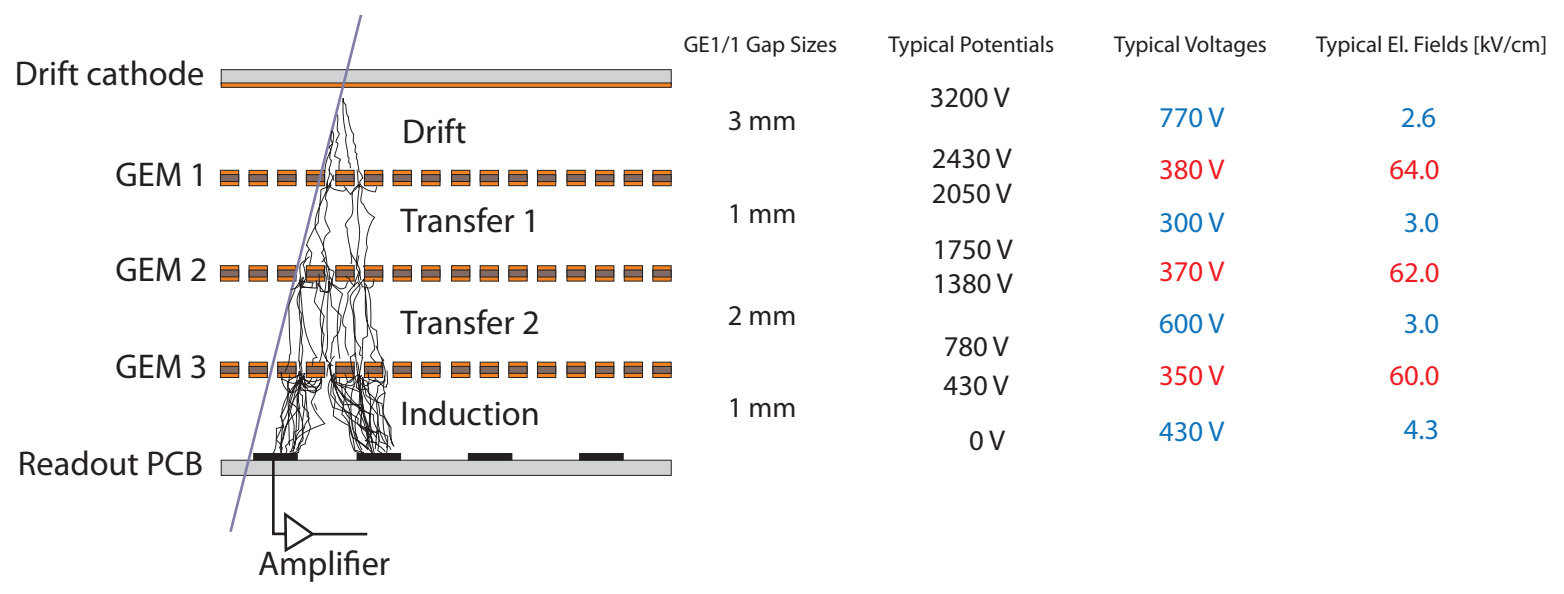

Figure 3.2.16: Triple-GEM operating principle [31].

of a few hundred volts. Each GEM foil has thin conductive layers of copper on each side and is perforated with hexagonal holes. The strong electric field inside the holes causes an avalanche of ionized particles. While each foil by itself has a modest gain, the combination of three foils yields a total amplification factor of up to 105.

The induced charges are then read out by sensitive electronics, segmented into 384 strips in $\phi$, which are in turn segmented into eight $\eta$ sectors. Each strip covers $450 \mu \mathrm{rad}$ in $\phi$, providing a finely segmented position measurement in the muon bending direction. As shown in Fig. 3.2.17, the on-chamber readout is performed by 24 custom VFAT ASIC (Application Specific Integrated Circuit) chips. Each chip reads out 128 strips in one $\eta$ sector. A pair of trapezoidal Triple-GEM chambers is combined to form a GEMINI chamber, which covers a $\sim 10^{\circ}$ sector and complements a single ME1/1 chamber. Due to the physical constraints of the CMS endcap, the 36 GEMINI in a ring alternate between long $(1.55<|\eta|<2.18)$ and short $(1.61<|\eta|<2.18)$ detectors. The first test of the CMS GEMs, the GEM demonstrator, was installed during the LHC EYETS (Extended Year-End Technical Stop) 2016-2017. My work preparing for the GEM upgrade and assisting the installation is described in Appendix A.2. 


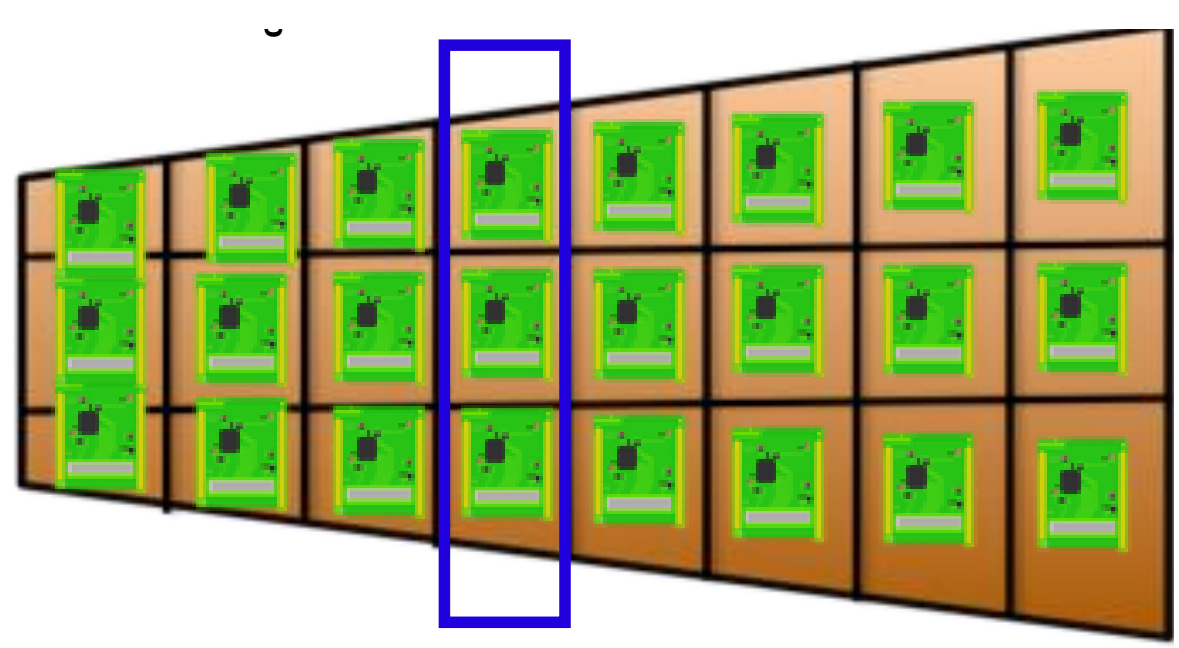

Figure 3.2.17: The 24 readout sectors of a GEM chamber [31].

\subsubsection{Trigger and Data Acquisition}

During the 2015 and 2016 data taking eras of Run II of the LHC, a peak instantaneous luminosity of $1.5 \times 10^{34} \mathrm{~cm}^{-2} \mathrm{~s}^{-1}$ was achieved, and up to 52 collisions per bunch crossing (pileup) were recorded in 2016 [32]. This high rate of events makes it challenging to keep all interesting physics events, given limitations in processing and data storage. Therefore, the CMS trigger system was designed in order to save collision events, which meet certain criteria, at a rate of $1 \mathrm{kHz}$ or less, out of the 40 million beam crossings that occur every second (one collision every $25 \mathrm{~ns}$ ). The CMS trigger architecture consists of two sequential levels: the Level-1 trigger (L1T) and the High Level Trigger (HLT), which are designed to reduce the total output event rate by a factor of $10^{6}$.

The L1T is instrumented with custom, programmable electronics, located partially on the detectors and partially in an underground control room $\sim 90 \mathrm{~m}$ from the CMS cavern. The architecture of the upgraded Run II L1T is shown in Fig. 3.2.18, It consists of two main parts, the L1 Calorimeter Trigger and the L1 muon trigger. The former receives information from the ECAL, HCAL, and HF subdetectors in order to compute energy sums and to reconstruct jet, hadronically decaying $\tau$, and $e / \gamma$ (electrons and photons) candidates. The latter combines three muon track finders - the barrel muon track finder (BMTF), the endcap 


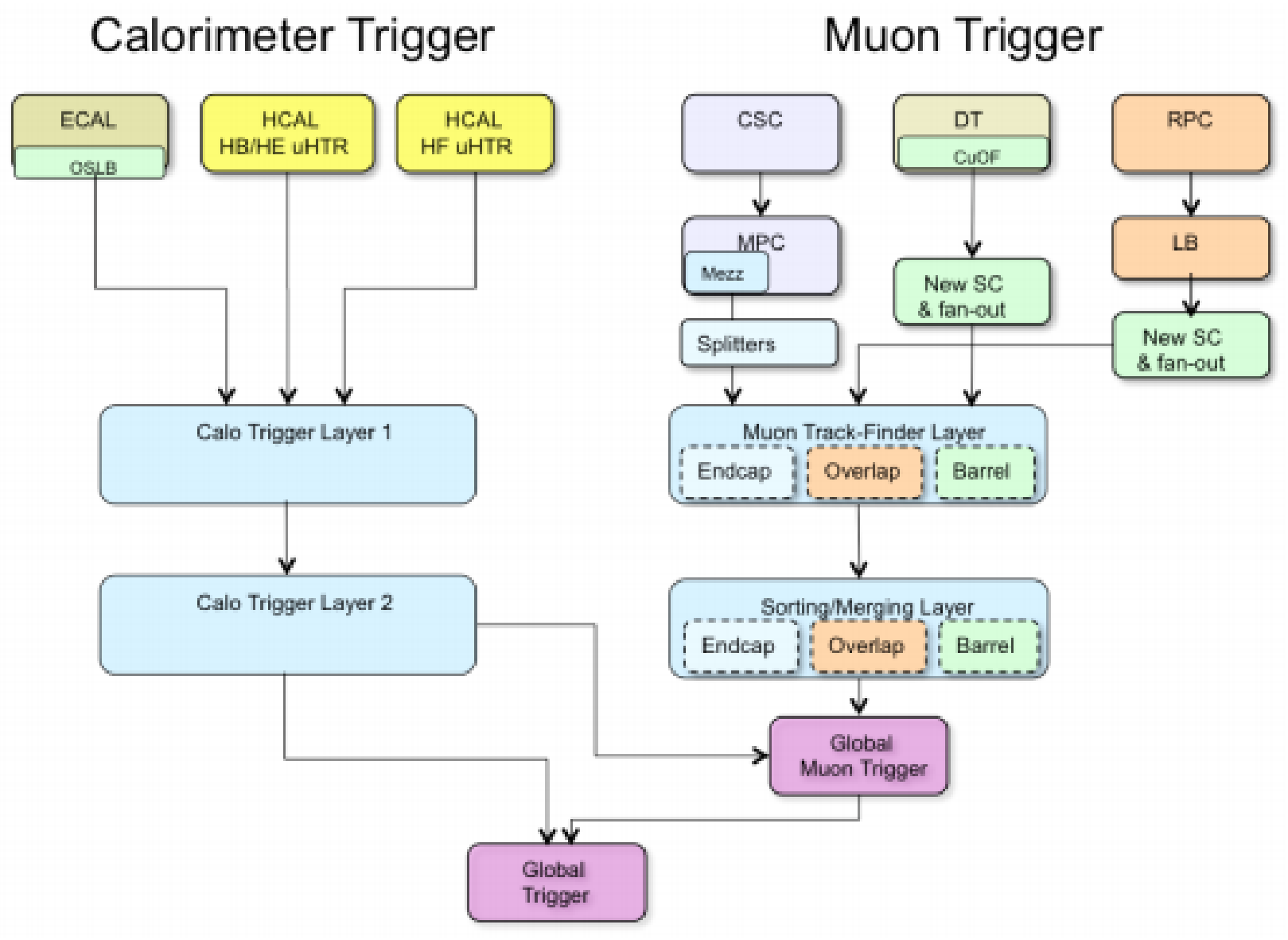

Figure 3.2.18: Schematic of the CMS Level-1 trigger, used for LHC Run II [32]. 
muon track finder (EMTF), and the overlap muon track finder (OMTF), covering the regions $|\eta|<0.83,|\eta|>1.24$, and $0.83<|\eta|<1.24$, respectively - whose output is sent to the Global Muon Trigger $(\mu \mathrm{GMT})$. The $\mu \mathrm{GMT}$ ranks muons by quality and $p_{T}$ and removes duplicates across track finder boundaries. It also uses information from the Calorimeter Trigger to compute the isolation (in $\Delta R$ ) of muon candidates.

Both the Calorimeter Trigger and $\mu$ GMT output their collections of physics objects to the micro Global Trigger ( $\mu \mathrm{GT}$ ), which combines their position, momentum, isolation, and quality in order to make the final trigger decision. This Level-1 Accept (L1A) is communicated to the CMS subdetectors through the Timing, Trigger, and Control (TTC) system [25]. The time between a bunch crossing and sending the trigger decision to the detector front-end electronics is about $3.8 \mu \mathrm{s}$. The L1T is designed to have an output rate of 100 $\mathrm{kHz}$, which translates to a maximum output rate of $30 \mathrm{kHz}$ in practice.

After passing the L1T, an event is passed to the CMS Data Acquisition (DAQ) system, the schematic of which is shown in Fig. 3.2.19. The event data is extracted from the subdetectors' front-end buffers, sent to the DAQ system by the Front-End Drivers (FEDs), and read in by the Front-end Read-out Links (FRLs). The event builder combines the different fragments from a single L1A into an event and sends it to one Filter Unit (FU) in the Event Filter, a computing farm of about 1000 processing nodes. The Event Filter performs Data Quality Monitoring (DQM) on the data and sends the events to the HLT.

The HLT is a software system, implemented on the filter farm node, which performs complex calculations on the full events to determine which ones contain physics processes of interest. The resulting stored events are separated into HLT paths, which are defined by the kinematic criteria the events are required to meet. For example, the PFHT800 path requires events to have $H_{T}>800 \mathrm{GeV}$, where $H_{T}$ is defined as the scalar sum of the $p_{T}$ of every jet in the event $\left(p_{T}>30 \mathrm{GeV}\right)$. This, and other HLT triggers, are used in the analysis presented in this thesis. After the HLT, the event rate is reduced to the desired rate, and the data are then sent to mass storage in the CERN data center. 


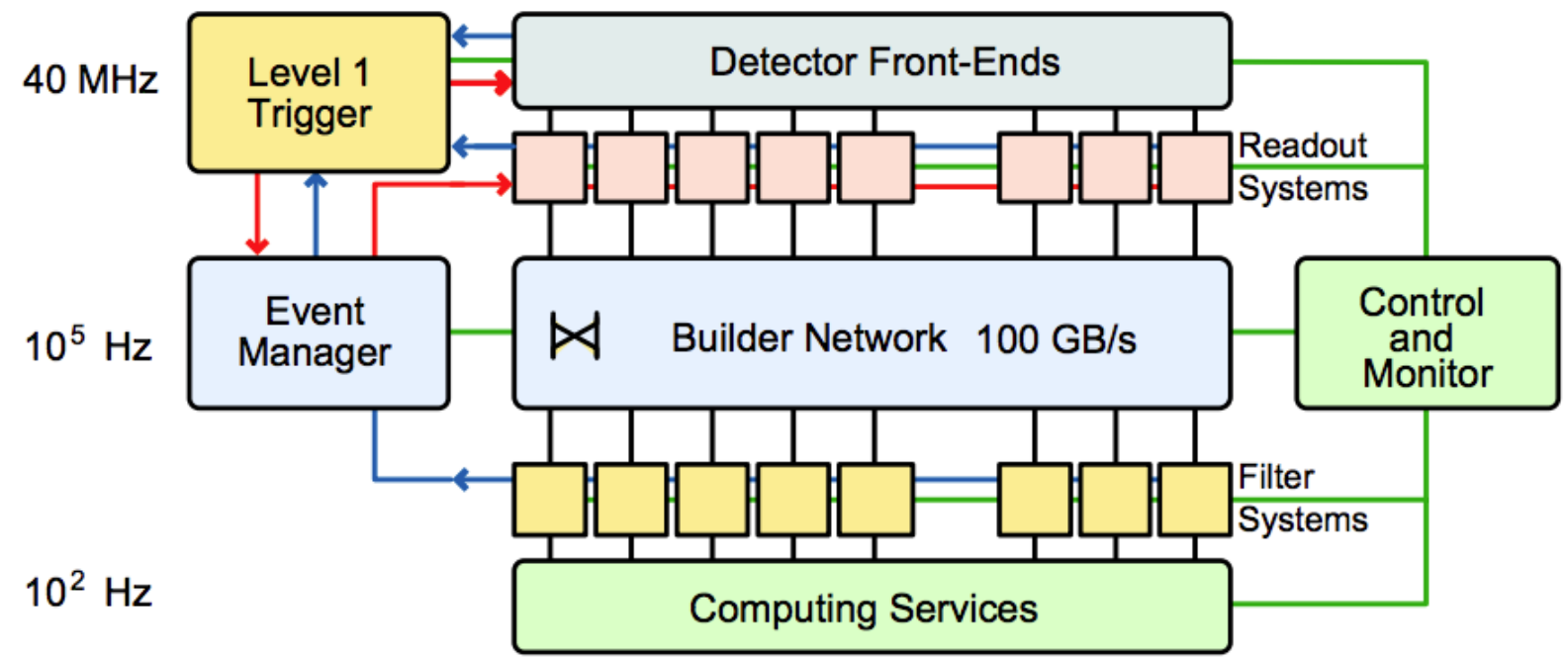

Figure 3.2.19: Schematic of the CMS DAQ system [25]. 


\section{Chapter 4}

\section{Event Reconstruction}

Once collision events have been recorded and stored by CMS, sets of pattern-recognition algorithms are used to reconstruct physics objects from the combined information from the different detector subsystems. These reconstructed objects will later be referred to as "reco" objects. The events are thereby transformed from tracks and energy deposits into a group of particles that originate from a single proton-proton collision point, or primary vertex. This chapter provides an overview of standard event reconstruction in CMS. A particular emphasis is placed on jet reconstruction, which is of particular importance to the physics analysis presented later in this dissertation.

\subsection{Particle Flow}

In CMS, as in many other modern multipurpose particle detectors, particles are identified based on the way they interact with the various detector layers, arranged in a cylinder around the beam axis. First, in the tracker, charged particles' paths (tracks) and origins (vertices) are reconstructed from "hits" in the active detector layers. The magnetic field in which the tracking detector is immersed bends the paths of the charged particles, allowing their charge and momenta to be measured. Next, electrons and photons deposit their energy in the ECAL. The resulting electromagnetic showers are recorded as energy clusters in neighboring 


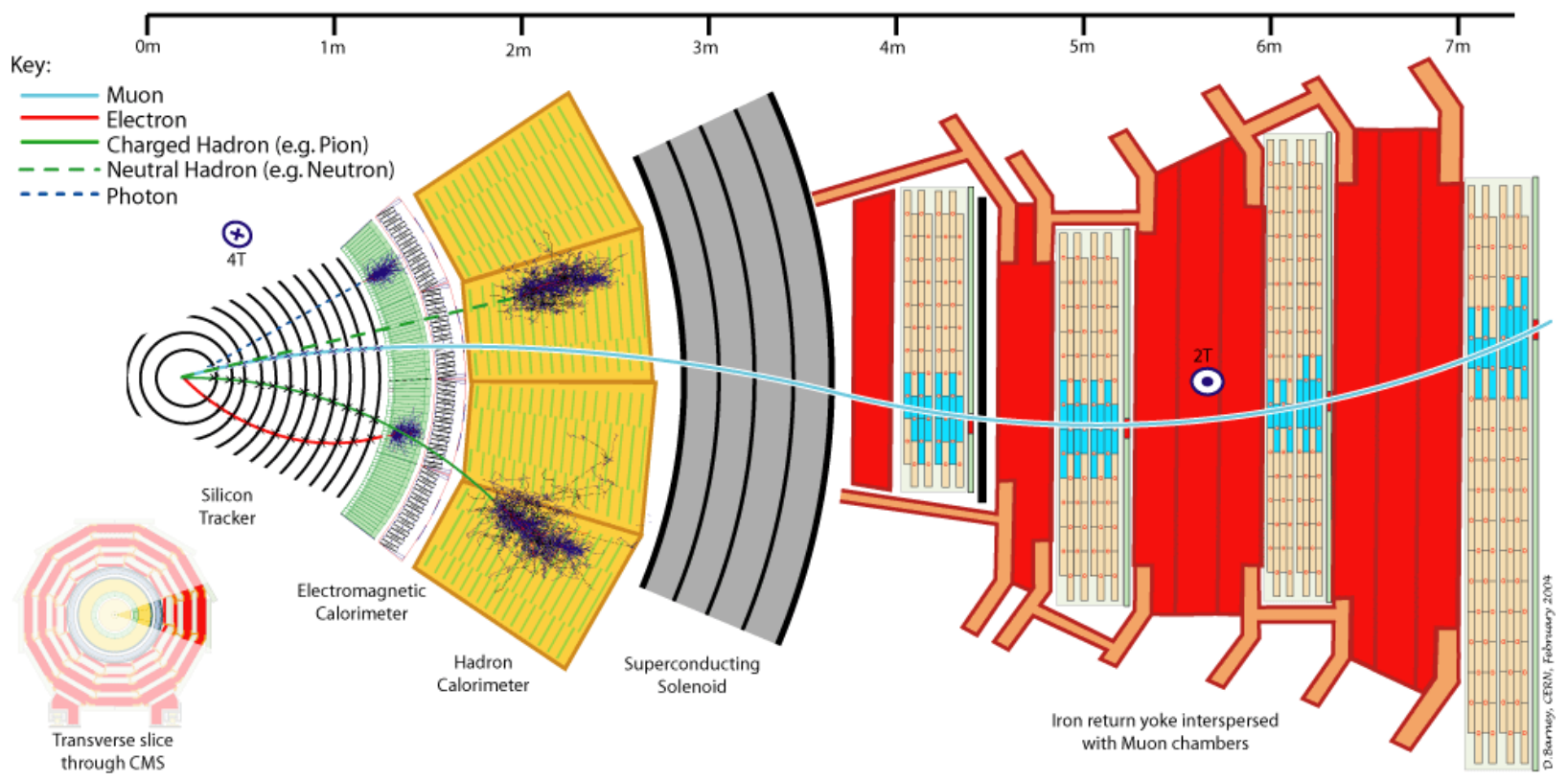

Figure 4.1.1: A transverse slice of the CMS detector, showing the interactions of the different types of particles with the different detector elements [33].

detector cells, which allow the direction and energy of the particles to be determined. Neutral and charged hadrons may start to shower in the ECAL, though their energy is not fully absorbed until they pass into the HCAL. As in the ECAL, energy clusters are used to measure the particles' energies and directions. While muons deposit little to no energy in the calorimeters, they then pass through additional tracking layers built specifically for muon detection. Neutrinos do not interact with the detectors at all and must be reconstructed from the missing transverse energy (MET) in the event (the total energy of the event in the $x-y$ plane should sum to zero). Figure 4.1.1 gives a simplified view of these interactions through a transverse slice of the CMS detector [33]. 
Traditionally, physics objects have been reconstructed using separate detector elements: jets and missing energy from the calorimeters, isolated photons and electrons from the ECAL, $b$ jet and hadronic $\tau$ decays from the tracker, and muons from the muon detectors. CMS, however, uses an improved reconstruction algorithm, called Particle Flow (PF), which combines the basic elements of the different detector layers into final state particles. Combining more tracks and energy measurements yields more precisely reconstructed particles.

The first step of Particle Flow reconstruction makes use of the link algorithm to connect the PF constituents from different subdetectors [33]. For a given event, this algorithm will test pairs of detector elements that are nearest neighbors in the $\eta-\phi$ plane. This restriction reduces the computing time to a manageable length, even for large numbers of particles. The specific requirements needed to link two detector elements depend on the subdetectors in question and are described in [33]. Once two elements are linked, the algorithm defines a distance between them, with closer elements rated as a higher quality link. Finally, PF blocks of detector elements are created, associated either through a direct link or an indirect link made through commonly linked elements.

Figure 4.1 .2 shows most of the possible link combinations that result in photons, charged hadrons, and neutral hadrons. For illustrative purposes, a jet with a $p_{T}$ of $65 \mathrm{GeV}$ and only five constituent particles $\left(K_{L}^{0}, \pi^{+}, \pi^{-}\right.$, and $\left.\pi^{0} \rightarrow \gamma \gamma\right)$ was simulated in the CMS detector. The track T1 is linked to the ECAL energy cluster E1 and to the HCAL energy clusters H1 (smaller link distance) and H2 (larger link distance). The track T2 is also linked to clusters $\mathrm{H} 1$ and $\mathrm{H} 2$ in the HCAL, but not to any ECAL clusters. A PF block with five PF elements is then created. In this block, T1, E1, and $\mathrm{H} 1$ correspond to the $\pi^{-}$, while $\mathrm{T} 2$ and $\mathrm{H} 2$ correspond to the $\pi^{+}$. There are three other ECAL clusters, which correspond to the neutral kaon and to the pair of photons resulting from the $\pi^{0}$ decay. As none of these clusters are linked to any track or other cluster, they each form their own PF block [33].

Generally, PF blocks do not contain elements from more than a few particles, and therefore PF computing time increases only linearly with particle multiplicity. The particle mul- 

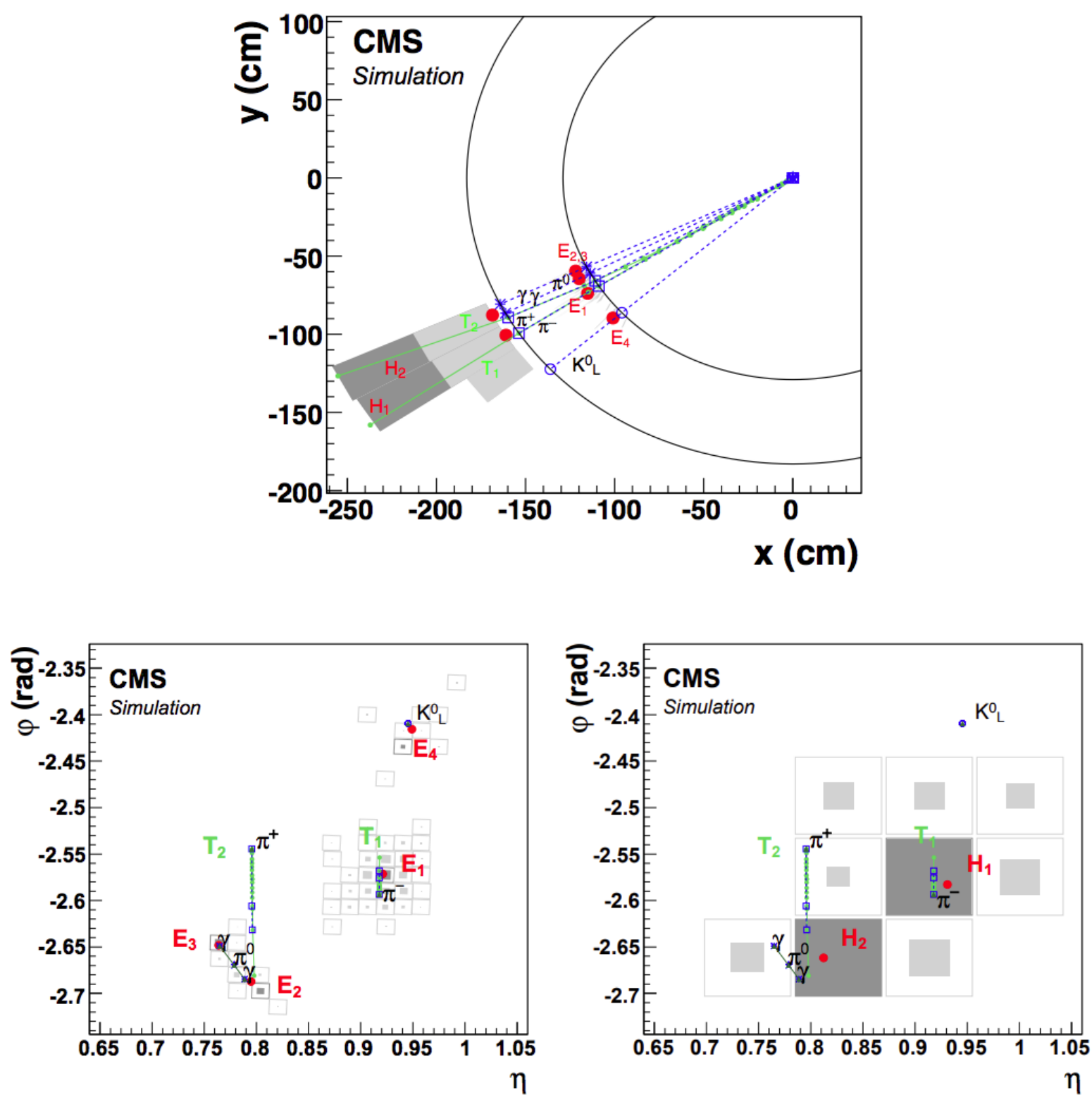

Figure 4.1.2: Event display of a PF jet made up of $K_{L}^{0}, \pi^{+}, \pi^{-}$, and $\pi^{0}$ particles. The jet is reconstructed by linking tracks and calorimeter clusters. The top illustration depicts the $x-y$ plane of the CMS detector, with the concentric circles indicating the calorimeter surfaces. The bottom left (right) display shows the $\eta-\phi$ surface of the ECAL (HCAL). The green solid lines show the charged particle trajectories, while the blue dashed lines show those of the neutral particles. The blue markers indicate the particles' impact positions on the calorimeter surfaces, while the red dots give calorimeter cluster positions. In the bottom two views, the cells included in these clusters are depicted as boxes, with the cells with the largest energy deposits in dark grey. In this specific event, the two charged pions $\left(\pi^{-}, \pi^{+}\right)$ are reconstructed as two charged-particle tracks $\mathrm{T}_{1,2}$, which point to the two HCAL clusters $\mathrm{H}_{1,2}$. While the $\pi^{+}$does not create an ECAL cluster, the $\pi^{-}$, the two photons from the $\pi^{0}$ decay, and the $K_{L}^{0}$ are detected as four ECAL clusters $\mathrm{E}_{1,2,3,4}$ [33]. 
tiplicity per unit of solid angle, as well as subdetector granularity, does affect the probability for the algorithm to link subdetector elements arising solely from a single particle. Additionally, the probability for all of the elements resulting from a given particle to be linked is primarily limited by the amount of material encountered before reaching the calorimeters and muon detectors. Traversing more material may lead to the emission of secondary particles and to small trajectory deviations due to multiple scattering.

The particle flow algorithm identifies particles in the following order [33]. First, muon candidates are identified using muon detector tracks, inner tracks, and calorimeter energy deposits. The corresponding PF elements are then removed from their PF block. Next, electrons and photons are identified, combining information from the inner tracker and calorimeters. The two particle types are identified at the same time, because electrons traversing the detector often emit bremsstrahlung radiation in the form of photons, which often convert to $\mathrm{e}^{+} \mathrm{e}^{-}$pairs, etc. Electron candidates should contain linked hits from both the inner tracker and calorimeters (primarily ECAL), while isolated photon candidates should contain only calorimeter hits, with no linked tracks. As with muon candidates, electron and photon candidates are removed from their respective PF blocks once identified.

Once muons, electrons, and isolated photons have been identified and removed from the appropriate PF blocks, clustering algorithms are used to group charged hadrons, neutral hadrons, nonisolated photons, and muons from charged hadron decays into jets. Jet clustering techniques will be discussed further in the next section. Finally, after all tracks and calorimeter clusters have been assigned to physics objects, the vector sum of the transverse energy of each event is calculated, and its negative is determined to be the MET of the event. 


\subsection{Jets}

\subsubsection{Jet Clustering}

Quarks and gluons produced in proton-proton collisions at the LHC result in collimated sprays of energetic particles known as jets. Ideally, reconstructed jets should contain all of the decay products from a single quark or gluon coming from the initial hard scatter. To do this, CMS uses pattern recognition algorithms, called jet clustering algorithms. These algorithms should be infrared and collinear (IRC) safe, meaning they should be insensitive to the emission of arbitrarily soft (low energy) or collinear particles [34]. Additionally, jet clustering algorithms should provide a repeatable method for combining constituent particles.

The jet algorithms considered in this dissertation are part of a group of related sequential recombination algorithms: $k_{T}$ [35], Cambridge/Aachen (CA) [34], and anti- $k_{T}(\mathrm{AK})$ [36]. To form a jet, the algorithms iterate over pairs of particles, considering the following quantities:

$$
d_{i j}=\min \left(k_{T, i}^{n}, k_{T, j}^{n}\right) \frac{\Delta R_{i j}^{2}}{R^{2}} d_{i B}=k_{T, i}^{n}
$$

where $\Delta R_{i j}^{2}=\left(y_{i}-y_{j}\right)^{2}+\left(\phi_{i}-\phi_{j}\right)^{2}$ is the distance between particles $i$ and $j$ in the $y$ - $\phi$ plane and $y_{i}, \phi_{i}$, and $k_{T, i}$ are the transverse rapidity, azimuthal angle, and transverse momentum of particle $i$, respectively [36]. The maximum allowed radius for a jet is represented by $R$, while $d_{i B}$ represents the distance between the beam and particle $i$. For the $k_{T}$, Cambridge/Aachen, and anti- $k_{T}$ algorithms, the respective values of $n$ are 2,0 , and -2 .

The clustering process identifies the minimum of all $d_{i j}$ and $d_{i B}$. If a $d_{i j}$ is the smallest, particles $i$ and $j$ are merged into a single jet. If instead $d_{i B}$ is the smallest, particle $i$ is a final jet and removed from the list of potential jet constituents. The algorithm continues forming jets until no particles are left.

The physical difference between the three algorithms lies in the $n$ parameter, which governs the relative power of the geometric vs energy scales. The simplest case is the CA algorithm, which preferentially clusters nearby particles, without consideration for their rel- 

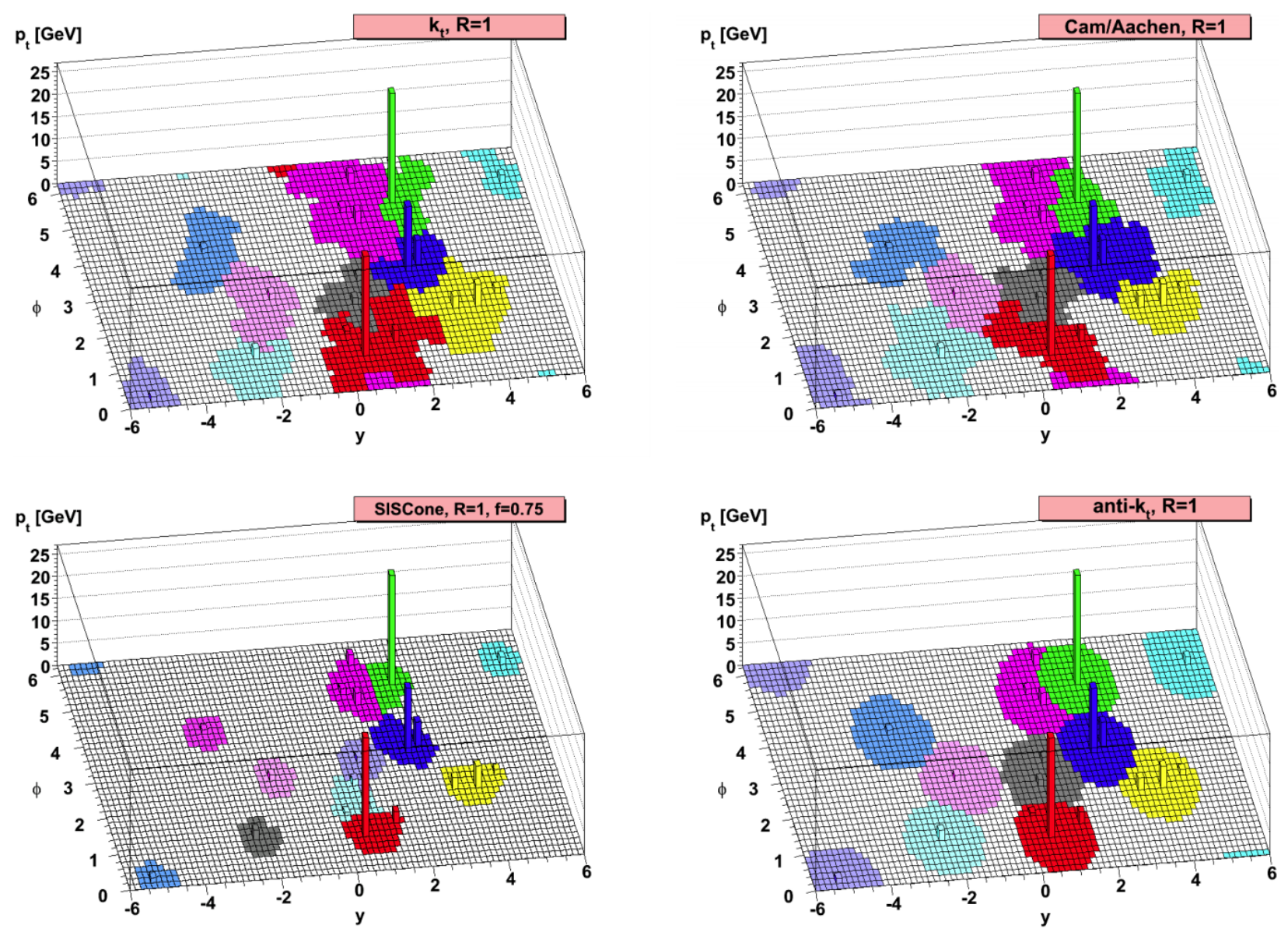

Figure 4.2.1: Jet shapes after different clustering parameters [36].

ative energies. The $k_{T}$ algorithm preferentially combines nearby low-momentum particles, while the anti- $k_{T}$ algorithm gives preference to the merging of nearby high momentum particles. Figure 4.2.1 shows an example of how the same set of particles would be clustered with these different algorithms. The shapes of the $k_{T}$ and CA jets fluctuate in response to the presence of soft (low-energy) particles, while the anti- $k_{T}$ algorithm gives rise to mostly conical jets that are centered on its hardest (highest energy) constituent.

The anti- $k_{T}$ algorithm is especially useful for analyses that focus on high energy jets, as they can be iteratively declustered to remove soft and wide angle radiation while still keeping the hard particle of interest. Such "jet grooming" techniques will be introduced in Chapter 5. In that section, both CA15 jets $(R=1.5)$ and $\operatorname{AK} 8(R=0.8)$ jets are used, while the $Z^{\prime}$ 
search only uses AK8 jets.

\subsubsection{Pileup Removal Algorithms}

Primary vertices are separated spatially along the beam axis and ranked by the quadratic sum of the $p_{T}$ of their tracks [33]. The primary vertex with the highest $\sum p_{T}^{2}$ is considered to be the hard-scatter vertex, and the others are called pileup vertices. During the jet clustering process, sometimes unwanted particles originating from pileup vertices are merged into the jet of interest. While there are many techniques used to remove these pileup particles, two methods of particular interest to this analysis are "pileup charged-hadron subtraction" (CHS) and "pileup per particle identification" (PUPPI).

Particle Flow CHS (PFCHS) makes use of the fact that some charged hadrons can be linked to tracks that point back to the scattering vertex from which they originate [33]. If a charged hadron is identified as coming from pileup, it is removed from the list of particles used to form physics objects. This technique, however, does not work for photons, neutral hadrons, and reco particles outside of the tracker acceptance. Fortunately, there is a relatively uniform $p_{T}$ density of pileup interactions in the $(\eta, \phi)$ plane. Therefore, in addition to PFCHS, the average $p_{T}$ contribution from pileup can be subtracted from CMS physics events.

A newer technique for pileup mitigation called PUPPI, which is used as an extension of PFCHS in CMS, aims to remove all pileup particles from jets. First, the variable $\alpha$ is computed for each particle $i$ in an event:

$$
\alpha_{i}=\log \sum_{j \in R_{\min } \leq \Delta R_{i j} \leq R_{0}} \frac{p_{T, j}}{\Delta R_{i j}}
$$

where $p_{T, j}$ is the transverse momentum of particle $j$ and $\Delta R_{i j}$ is the distance between particles $i$ and $j$ in $(\eta, \phi)$ [37]. $R_{0}$ and $R_{\min }$ define the outer and inner boundaries of a cone, centered on particle $i$, within which other particles are included in the sum. Figure 4.2 .2 shows the $\alpha_{i}$ distributions for particles in both the central and forward regions of the 

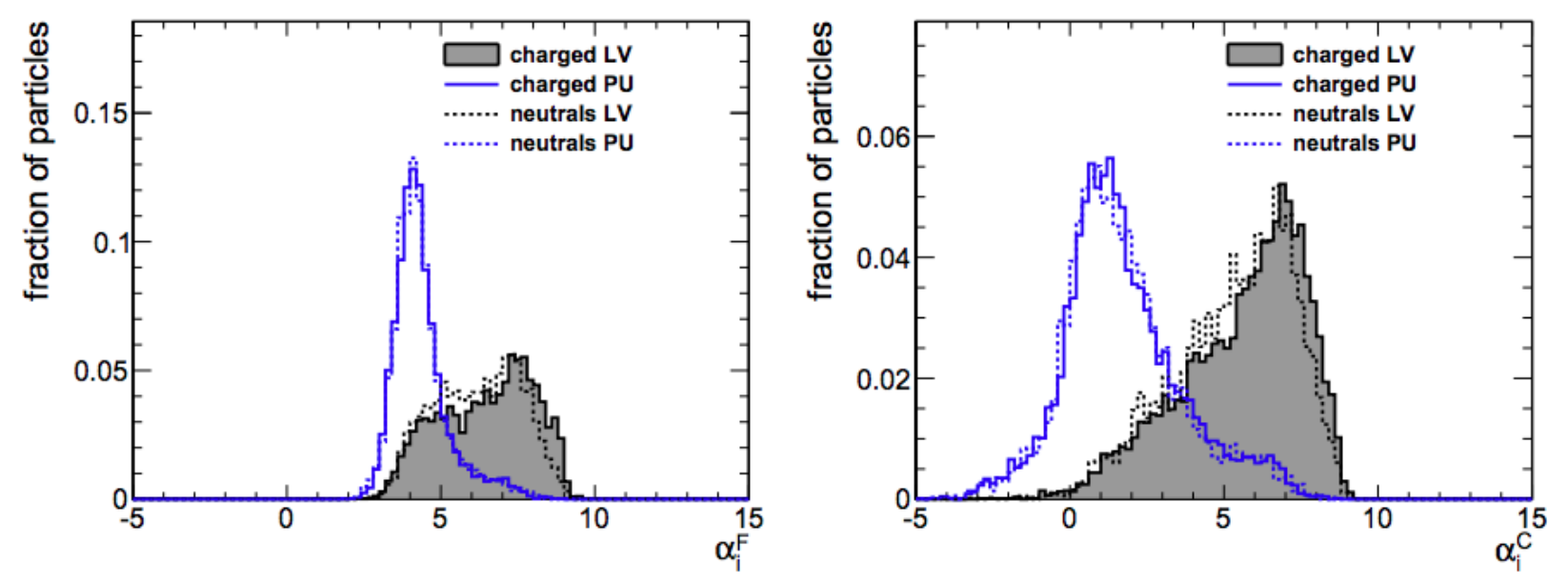

Figure 4.2.2: The distribution of $\alpha_{i}$ for particles in the forward region of CMS, $\eta>2.5$, (left) and particles in the central region, $\eta<2.5$, (right) [37]

\section{CMS detector.}

Once $\alpha_{i}$ has been calculated, each particle is assigned a weight by comparing its $\alpha$ value to the median of the charged pileup $\alpha$ distribution. This weight indicates how likely a particle is to come from pileup and ranges in value from zero (most pileup-like) to one (most hardscatter-like). The weights are used to rescale the particles' four-momenta, $\left(E, p_{x}, p_{y}, p_{z}\right)$. Then, particles with very small rescaled $p_{T}$ and/or very small weights are discarded. These PUPPI particles can then be used as the input to e.g. a jet clustering algorithm. 


\section{Chapter 5}

\section{Top Tagging Studies}

In the analysis discussed in this dissertation, $Z^{\prime}$ bosons and RS KK gluons decay to top quark pairs, which in turn decay hadronically $(t \rightarrow W b, W \rightarrow q \bar{q})$. Due to the high mass (1-5 TeV) of the resonances, each top is highly Lorentz-boosted, causing its decay products to be highly collimated. For a top quark with a given $p_{T}$, these decay products are generally

contained within a cone of $\Delta R=\frac{2 m}{p_{T}}$, where $m$ is the mass of the top quark. Therefore, a jet clustering algorithm with a distance parameter of $R=\Delta R$ will be able to collect all of the decay products of a "top jet".

Due to the high rates of QCD multijet production during $13 \mathrm{TeV}$ proton-proton collisions at the LHC, many techniques have been developed to distinguish these backgrounds, which originate from light quarks or gluons, from top jets, which have large invariant mass and a distinctive three-pronged substructure. One such technique is mass grooming, which is used to remove soft and wide angle radiation from within a jet, coming from pileup, parton shower activity, or underlying events. In this chapter, trimming [38], pruning [39], filtering [40], and softdrop [41] grooming methods are discussed.

Other substructure observables are also used to discriminate between top and QCD jets. Examples include n-subjettiness [42], used to identify the number of subjets inside of a jet, and Q-jet volatility [42], which measures the stability of the jet mass when subjected to 
randomized clustering of the jet constituents. Combined taggers, which are designed to perform a complete reconstruction of the kinematics of the top decay, include the CMS Top Tagger (CMSTT) [43, 44], the HEPTopTagger Version 2 (HTT V2) [45, and shower deconstruction [46, 47].

A selection of these algorithms are briefly described in this chapter, followed by a study of their performance for high- $p_{T}$ jets $\left(p_{T}>400 \mathrm{GeV}\right)$. Combinations of different tagging tools are considered, and their stability is compared as a function of $p_{T}$ and number of pileup vertices. Example working points are used to examine efficiency of both signal selection and background rejection.

This chapter draws on the work documented in [48], to which I was a major contributor. Earlier CMS top tagging studies are documented in [49]. The studies presented were undertaken not only for the $Z^{\prime}$ search but also to set recommended working points for all CMS LHC Run II analyses.

\subsection{Simulated Samples, Object Definitions and Event Selection}

Top-tagger performance is evaluated for QCD and hadronic top jets with $p_{T}$ in the ranges 470-1400 GeV, using simulated collision data at a proton-proton center-of-mass energy of $\sqrt{s}=13 \mathrm{TeV}$. Samples of $Z^{\prime}$ resonances, ranging in mass from 1-3 $\mathrm{TeV}$ and decaying exclusively to $t \bar{t}$, are used to provide the boosted-top signal. These $Z^{\prime}$ samples are simulated with MADGRAPH5 [50], which is interfaced with PYTHIA8.2 [51] for the hadronization. The resonances are narrow, with widths equal to $1 \%$ of their masses. The QCD multijet background samples are generated with PYTHIA8.2 and are separated into bins in $p_{T}$.

AK8 PFCHS jets are primarily used for the taggers considered in this chapter, as nearly $100 \%$ of the decay products of a top with $p_{T}>470 \mathrm{GeV}$ can be collected in a jet with a cone of $R=0.8$. The one exception is the HEPTopTagger, described in section 5.2, which takes 


\begin{tabular}{cc|c|ccc|c|c|c|c}
\multicolumn{2}{c|}{$p_{T}$ range $[\mathrm{GeV}]$} & Max. $|\eta|$ & \multicolumn{2}{|c|}{$\mathrm{QCD}$} & $m_{Z^{\prime}}[\mathrm{TeV}]$ & $R$ & $\Delta R(\mathrm{p}, \mathrm{jet})$ & $\max (\Delta R(\mathrm{t}, \mathrm{q}))$ \\
\hline $470-600$ & 2.1 & 470 & - & 600 & 1.25 & 0.8 & 0.6 & 0.6 \\
$600-800$ & 2.1 & 600 & - & 800 & 2.0 & 0.8 & 0.6 & 0.6 \\
$800-1000$ & 1.5 & 800 & - & 1000 & 2.0 & 0.8 & 0.6 & 0.6 \\
$1000-1400$ & 1.5 & $1000-1400$ & 3.0 & 0.8 & 0.6 & 0.6
\end{tabular}

Table 5.1: Overview of the selection criteria for top tagging performance studies. The first column gives the $p_{T}$ bins into which the samples are separated, while the second column gives the corresponding $|\eta|$ requirements. The QCD samples are already $p_{T}$-binned, while the signal samples are denoted by the $Z^{\prime}$ mass. The signal samples are grouped into the $p_{T}$ ranges most compatible with the $p_{T}$ distributions of their top quark daughters. In the last three columns, $R$ denotes the reco jet cone size, $\Delta R(\mathrm{t}, \mathrm{q})$ gives the matching criterion, and $\Delta R(\mathrm{t}, \mathrm{q})$ gives the maximum distance between the top quark and its constituent quarks [48].

CA15 PFCHS jets as its input. As explained in the previous chapter, CHS is applied to the particle flow jets in order to mitigate pileup. In addition to the application of CHS, selected leptons are removed before the jets are reconstructed.

In order to ensure that the studies are performed on the desired objects, all jets are required to be matched to a generator-level object originating from the hard scatter. Signal jets are matched to generator-level hadronic top quarks, while background jets are matched to generator-level partons $(\mathrm{u}, \mathrm{d}, \mathrm{s}, \mathrm{c}, \mathrm{b}$ and gluon). The matching is done by selecting the closest jet, within $\Delta R<0.6$, to a generator-level top quark or parton.

Due to the large $p_{T}$ range of samples considered, the various top taggers are studied in bins of $p_{T}$, determined by the transverse momenta of the generator-level top quarks and partons. Once the samples have been separated into $p_{T}$ bins, a weight is assigned to each generatorlevel object, resulting in approximately flat distributions in $p_{T^{-}}$and $\eta$-space. Different $|\eta|$ selection criteria are applied for different $p_{T}$ bins, because the top-jet candidate distribution becomes more central at higher values of $p_{T}$. Additionally, in order to ensure that all three quarks from the top quark decay are contained within the larger jet, a restriction is placed on the $\mathrm{b}$ quark and the two quarks originating from the $\mathrm{W}$ decay: $\max (\Delta R(\mathrm{t}, \mathrm{q}))<0.6$. This restriction is only enforced for the signal selection. An overview of these selection requirements is shown in Table 5.1 . 


\subsection{Top Tagging Methods}

\subsubsection{Groomed Masses}

The most straightforward way to discriminate between jets originating from top quarks and those from light quarks and gluons is to use the invariant mass of the jets. Jet invariant mass is calculated as the combined invariant mass of the jet constituents. It should be much larger for top jets than QCD jets, due to the large mass of the top quark. Unfortunately, contributions from the underlying event, pileup, and initial state radiation can substantially increase the reconstructed jet mass, which should be nearly zero for light quark and gluon jets. This is especially problematic in boosted object identification, where more pileup is clustered into the jets due to the large cone sizes $(0.8,1.5)$ used.

Fortunately, jet grooming methods have been developed to remove these additional contributions to the reconstructed jet mass. The earliest such tools include trimming [38] $\left(m_{\operatorname{Tr}}\right)$ and filtering [40] $\left(m_{\text {Filt }}\right)$. In both methods, the constituents of the initial jet are reclustered into subjets with a smaller distance parameter $r$ (0.2 in this case). When the trimming algorithm is used, only the subjets that meet the criterion $p_{T, \text { subjet }}>f \cdot p_{T, \text { jet }}$ are kept, where $f$ is a constant. The filtering algorithm, instead, sorts the subjets in order of $p_{T}$ and only keeps the leading $n$ subjets. In both cases, the remaining subjets are combined, as a four-vector-sum, to form the new groomed jet.

Pruning [39] $\left(m_{\mathrm{Pr}}\right)$, on the other hand, is a reclustering procedure, removing jet constituents one at a time. Using all of its constituents, the clustering of the initial jet is repeated, with additional requirements. For two objects $i$ and $j$ to be combined into object $p$, the following conditions must be met:

$$
z=\frac{\min \left(p_{T i}, p_{T j}\right)}{p_{T p}}<z_{c u t} \text { and } \Delta R_{i j}>r_{c u t}
$$

where $p_{T x}$ is the transverse momentum of object $x$ and $\Delta R_{i j}$ denotes the distances between objects $i$ and $j$. Two parameters are used to suppress soft and wide-angle radiation, respec- 
tively: $z_{c u t}$ and $r_{c u t}$. If the conditions are not met, the lower- $p_{T}$ object is discarded. The clustering process continues until all constituents have either been removed or clustered into the new groomed jet.

The last mass groomer considered in this chapter is soft drop [52, 41] $\left(m_{\mathrm{SD}}\right)$, which iteratively declusters jets to remove soft and wide-angle radiation. Step by step, a jet's clustering is reverted, separating the jet $j$ into two subjets $j_{1}$ and $j_{2}$ at each stage. If the softdrop condition is met:

$$
\frac{\min \left(p_{T 1}, p_{T 2}\right)}{p_{T 1}+p_{T 2}}>z_{c u t} \cdot\left(\frac{\Delta R_{12}}{R}\right)^{\beta},
$$

then the procedure stops with final jet $j$. If not, the declustering continues, discarding the lower $p_{T}$ subjet and relabeling the higher $p_{T}$ one as $j$. The tunable parameters $z_{c u t}$ and $\beta$ used to set the strength of fractional $p_{T}$ and collinear radiation selection, respectively.

\subsubsection{N-Subjettiness}

Another useful tool for distinguishing between signal and background is the distribution of energy within a jet. The energy in hadronic top jets is expected to be deposited in three main regions, corresponding to the three quarks resulting from the top decay. QCD jets, on the other hand, are expected to have only one or two such regions, or subjets.

$\mathrm{N}$-subjettiness $\tau_{N}$ is an algorithm [42] designed to determine the consistency of a jet to have $N$ (or fewer) subjets. Jet axes are found for an $N$ subjet hypothesis, using the one-pass optimization algorithm. The consistency of the particle flow candidates with the individual axes is then determined, and n-subjettiness is calculated according to the following formula:

$$
\tau_{N}=\frac{\sum_{i \in \text { particles }} p_{T}^{i} \cdot \min \left(\Delta R_{1, i}, \Delta R_{2, i}, \ldots, \Delta R_{N, i}\right)}{\sum_{i \in \text { particles }} p_{T}^{i} \cdot R_{j e t}} .
$$

Here, the quantities $\Delta R_{j, i}$ represent the angular distances between particle $i$ and subjet axis 
$j$, where $j$ ranges between 1 and $N$ to compute n-subjettiness. The normalization factor in the denominator uses $R_{\text {jet }}$, the distance parameter used for jet clustering ( 0.8 in this case).

Jets with low values of $\tau_{N}$ have particle deposits that are more aligned with the hypothesized number and position of the subjet axes, while higher values of $\tau_{N}$ are found for jets inconsistent with a given hypothesis. Additional discrimination between signal and background jets can be obtained by taking ratios of successive $\tau_{N}$ values. The ratio of 3 -subjettiness to 2-subjettiness is used to increase identification efficiency and background rejection of top quark candidates.

\subsection{3 b Quark Tagging}

One important feature of a top jet is the presence of a subjet originating from the decay of a B hadron. Depending on their initial momenta, the lifetime of $\mathrm{B}$ hadrons is $\sim 1.5 \mathrm{ps}$, causing them to decay at a distance of a few $\mathrm{mm}$ to one $\mathrm{cm}$ from the initial top decay vertex. In the inner tracker, it is possible to reconstruct this secondary vertex. Additionally, the light quark and gluon jets associated with QCD backgrounds are not expected to contain such secondary vertices. Therefore, the presence of a subjet originating from a secondary vertex is a powerful tool for separating top jets and QCD jets. To this end, CMS employs a multivariate discriminator called combined secondary vertex version 2 (CSVv2) [53]. In this dissertation, softdrop subjets are used as inputs to the CSVv2 algorithm.

\subsubsection{CMS Top Tagger}

The CMS top tagger (CMSTT) [43, 44, 49] is a combined tagger that reverses the original pair-wise clustering of a jet, step by step, in order to find the initial jet. The declustering operates in two steps. First, in the primary decomposition, the input jet is declustered into two subjets. Then, in the secondary decomposition, an attempt is made to decluster each subjet. Selection criteria on the angular distances and transverse momenta of the subjets are applied in order to veto declusterings that are too close or too low in $p_{T}$. 
The CMSTT provides several discriminating variables: jet mass $m_{\text {jet }}$, the number of subjets $N_{\text {subjets }}$, and the minimum pairwise mass $m_{\min }$. This last variable is used as a proxy for the $W$ boson mass and is defined as the minimum invariant mass of all possible pairwise combinations of the three $p_{T}$-leading subjets. The Run-I $Z^{\prime} \rightarrow t \bar{t}$ analysis employed the CMSTT to identify top jets.

\subsubsection{HEPTopTagger}

Another combined tagger, the HepTopTagger [54, 55, 45] version 2 (HTT V2) is a multi-stage tagging algorithm. First, it declusters a CA15 jet $j$ into subjets $j_{1}$ and $j_{2}$ (where $j_{1}$ is the more massive of the two), reversing the original clustering if $m_{j 1}<0.8 m_{j}$, both subjets are kept for further analysis; if not, $j_{2}$ is discarded.

The declustering process is repeated for the subjets until all have a mass less than 30 $\mathrm{GeV}$. If fewer than three subjets remain, the top candidate is discarded. Otherwise, the three $p_{T^{-}}$leading subjets are sent to a filtering step: $R_{f i l t}=\min \left(0.3, \Delta R_{j k} / 2\right)$, after which the five $p_{T}$-leading subjets are kept. These are then reclustered into three subjets, corresponding to the three subjets in a top jet. If no combination can be found in which all three subjets have $p_{T}>30 \mathrm{GeV}$, the top candidate is discarded.

The discriminating variables obtained from this algorithm included the top quark mass $m_{123}$, i.e. the invariant mass of the three subjets, and $f_{R e c}$, the ratio between the reconstructed $W$ and top masses:

$$
f_{\text {Rec }}=\min _{i j}\left|\frac{\frac{m_{i j}}{m_{123}}}{\frac{m_{W}}{m_{t}}}-1\right|,
$$

where $m_{i j}$ is the invariant mass of subjets $i$ and $j$ and $m_{W}\left(m_{t}\right)$ is the true mass of the W boson (top quark).

Additionally, in the optimal-R approach, HTT V2 searches for an optimal cone size for the reconstructed top quark as a function of $p_{T}$. Testing cone sizes in descending order from 
$R=1.5$ to $R=0.5$, the previously described procedure is applied. The optimal cone size $\left(R_{\text {opt }}\right)$ is determined to be the smallest possible value of $R$ that results in a jet whose mass is less than $20 \%$ different from that found with $R=1.5$.

Therefore, HTT V2 provides another discriminating variable: $\Delta R_{o p t}=R_{\text {opt }}-R_{o p t}^{c a l c}$. This is the difference between a given jet's optimal cone size $R_{\text {opt }}$ and the expected value $R_{\text {opt }}^{\text {calc }}$, calculated as a function of $p_{T}$ for a sample of pure top jets.

\subsubsection{Shower Deconstruction}

Shower deconstruction [46, 47] is also a combined tagger which, unlike the previous two, provides a single likelihood-based discriminating variable. First, the input jet is reclustered with the $k_{T}$ algorithm into up to nine "microjets." The term microjet is used, because the reclustering is done with a small distance parameter, $M J R=0.1$ in this case.

The set of four momenta of the resulting microjets are denoted as $\{p\}_{N}=\left\{p_{1}, p_{2}, \ldots, p_{N}\right\}$. Then, the probability for a simplified parton shower Monte Carlo to produce $\{p\}_{N}$, given the background or signal hypothesis is calculated. The resulting signal (background) likelihood is denoted as $P\left(\{p\}_{N} \mid S\right)\left(P\left(\{p\}_{N} \mid B\right)\right)$.

The final shower deconstruction discriminating variable is defined as:

$$
\chi=\frac{P\left(\{p\}_{N} \mid S\right)}{P\left(\{p\}_{N} \mid B\right)} .
$$

where high values of $\chi$ indicate a more signal-like (top-like) jet, while low values of $X$ indicate a more background-like (QCD-like) jet. 


\subsection{Distribution of Top Tagging Discriminating Vari- ables}

This section examines distributions of discriminating variables described in Section 5.2. The $p_{T}$ and $\eta$ cuts and flattening weights, described in Section 5.1, are applied to all plots. The percentages reported in the plot legends indicate the number of entries plotted in each histogram, with respect to the fiducial selection. This efficiency results from generator-level matching, jet reconstruction, boosted algorithm, and preselection efficiencies, as well as the choice of $\mathrm{x}$-axis range.

\subsubsection{Mass and N-subjettiness}

Figure 5.3.1 shows distributions of AK8 jet masses in various $p_{T}$ bins for both QCD and boosted hadronic top jets. Compared to the ungroomed jets, jets with pruning and soft drop applied show a marked improvement in the discriminating power of the jet mass variable. Additionally, a much better stability across $p_{T}$ bins is seen for the groomed jet masses. This separation is slightly better for the softdrop mass, where the QCD mass spectrum is pushed closer to zero.

In both the pruned and softdrop mass signal spectra, a clear peak can be seen near the top mass value. A second peak is visible near the $W$ mass, due to top candidates whose b quark is not captured inside the $R=0.8$ jet cone. This feature is most prominent for the lowest $p_{T}$ bin, as expected. Applying the merged-top requirement removes this $W$-mass peak.

Figure 5.3 .2 shows the ungroomed $\tau_{3} / \tau_{2}$ distribution for signal and background jets. Unsurprisingly, the distribution of background jets tends toward lower values of $\tau_{3} / \tau_{2}$, while top jets tend toward higher values. Even after a cut on softdrop mass has been applied (110 $<m_{S D}$. $\left.<210 \mathrm{GeV}\right)$, good discrimination power of the $\tau_{3} / \tau_{2}$ variable is seen. Good stability as a function of $p_{T}$ is also observed. 

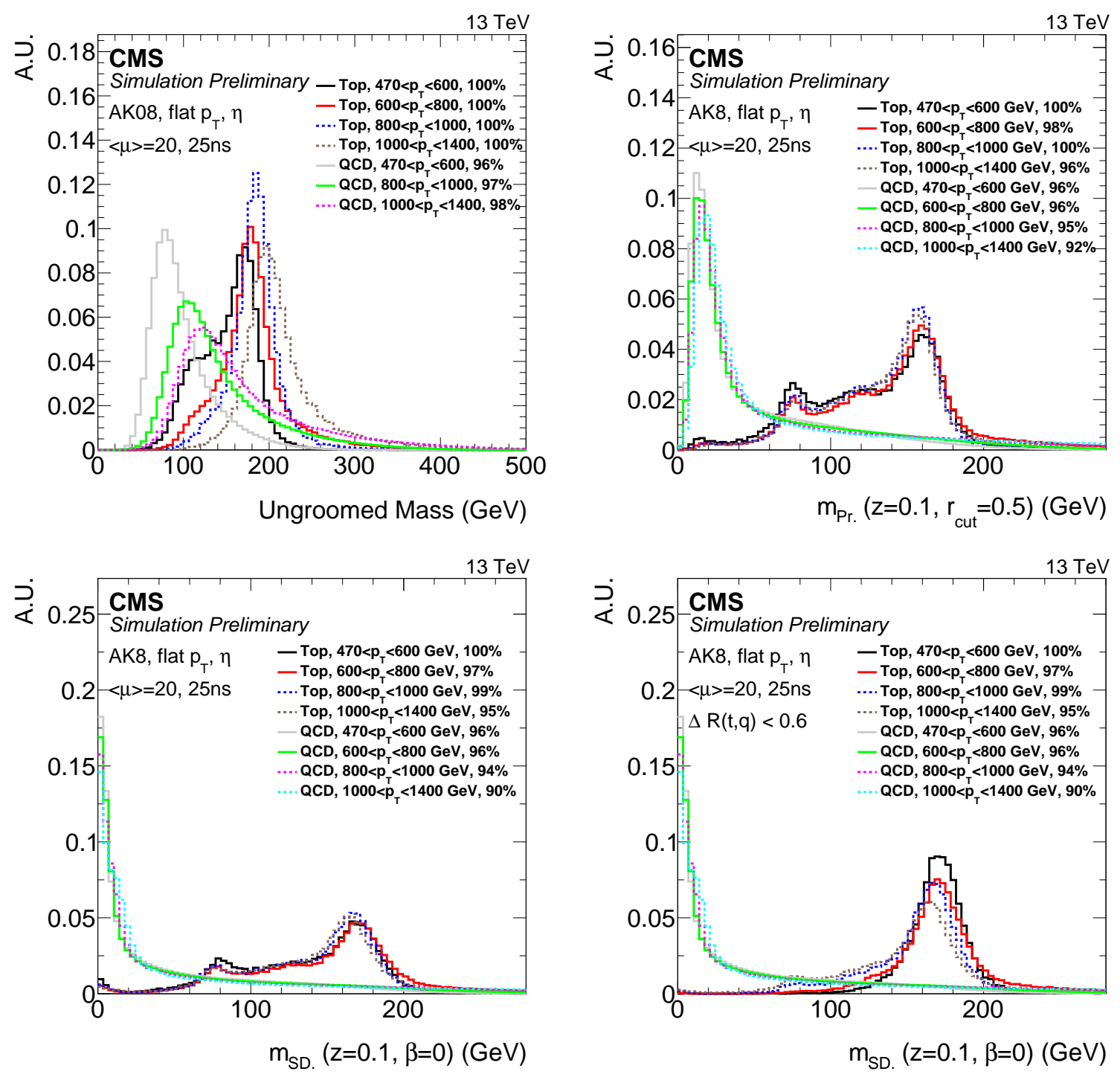

Figure 5.3.1: Distribution of AK8 jet masses with different groomers applied: no grooming - top left, pruning $\left(z=0.1, r_{c u t}=0.5\right)$ - top right, soft $\operatorname{drop}(z=0.2, \beta=1)$ - bottom row. The bottom left (right) shows the softdrop mass distribution without (with) the merged-top requirement. The percentages in the legends indicate the number of entries plotted in each histogram, with respect to the fiducial selection. Events were generated with an average of $\langle\mu\rangle=20$ pileup interactions and a 25-ns spacing between proton bunches [48]. 


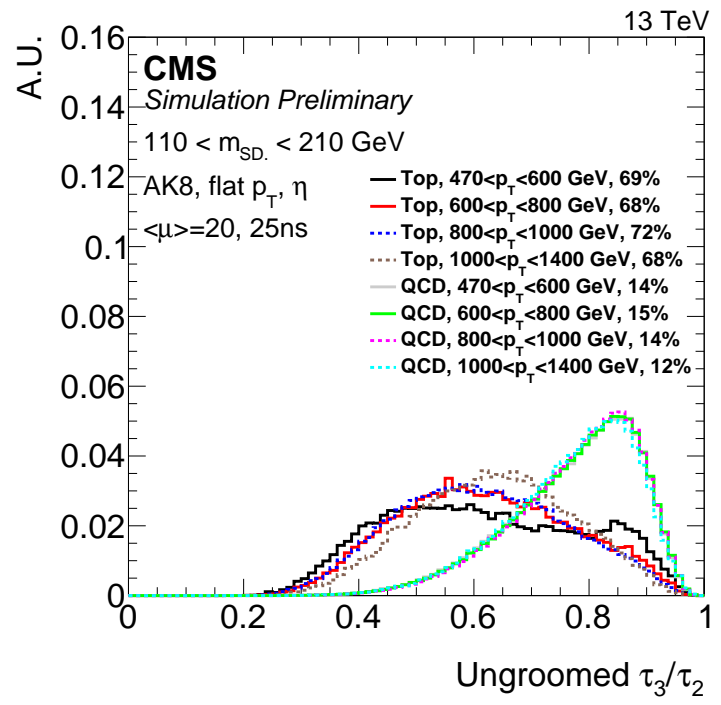

Figure 5.3.2: Distribution of ungroomed AK8 $\tau_{3} / \tau_{2}$, after a selection on softdrop mass has been applied $\left(110<m_{S D}<210 \mathrm{GeV}\right)$. The percentages in the legend indicate the number of entries plotted in each histogram, with respect to the fiducial selection. Events were generated with an average of $\langle\mu\rangle=20$ pileup interactions and a 25-ns spacing between proton bunches [48].

\subsubsection{Combined Tagger Variables}

The three most important HTT decision variables are shown in Fig. 5.3.3, In the mass plot, the top jet masses peak around 150, while the QCD peak is around $40 \mathrm{GeV}$. Due to the fact that this method automatically rejects top candidates that are not fully merged, a very minimal bump is seen near the $W$ mass in the signal distribution.

The $f_{\text {Rec }}$ distribution is quite peaked at zero for signal jets and is much flatter for background jets. The $\Delta R_{\text {opt }}$ distributions are peaked at zero, with the top jets displaying a sharper peak than the QCD jets and the sharpness of the peak increasing with $p_{T}$ for both signal and background samples. The mass and $f_{\text {Rec }}$ distributions, on the other hand, show very nice stability with respect to $p_{T}$. All HTT V2 variables exhibit the low efficiency of the HTT algorithm, due to the many stages at which top candidates are rejected.

Figure 5.3.3 also shows the distribution of the shower deconstruction variable $\chi$. Top jets peak near $\log (\chi) \approx 5$, while QCD jets peak near 0 . The efficiency of this variable can also 
be quite low, because the algorithm fails for some jets. The $p_{T}$ stability of the $\chi$ variable is not as good as some of the other variables considered.

\subsection{Tagger Combinations}

As the first step in comparing the performance of the different variables and taggers, single variable ROC (receiver operating characteristic) curves, shown on the left of Fig. 5.4.1, are considered. These curves are produced with the Toolkit for Multivariate Data Analysis with ROOT (TMVA) [56] package. Each curve shows the background mistag rate $\varepsilon_{B}$ vs signal efficiency $\varepsilon_{S}$ for a given observable. Each point on a given curve represents a cut-based selection window applied to that observable. The efficiency is defined as the number of jets that pass a given cut with respect to the total number that pass the initial selection criteria described in Section 5.1. The results are shown for one of the middle $p t$ bins, 800 $\mathrm{GeV}<p_{T}<1000 \mathrm{GeV}$.

Shower deconstruction by far outperforms the other variables, by at least a factor of three for most of the phase space considered. The next most performant variables are nsubjettiness, at low signal efficiencies, and HTT V2 mass, at high effciencies.

Next, combinations of variables are considered. A $z$-score diagram (Fig. 5.4.2) is used to determine the combinations that provide the best signal/background discrimination power. The $z$-score is defined as $1 / \varepsilon_{B}$ when the signal efficiency is equal to $30 \%$. A projective likelihood estimator is used for the single-variable entries on the diagonal, while a Boosted Decision Tree is used for the off-diagonal entries.

The diagonal entries in the $z$-score diagram can be compared to the single-variable ROC curves. Considering a signal efficiency of $30 \%$, the shower deconstruction variable $\chi$ is the most performant, with a $z$-score of 385 and a background mistag rate of $\sim 0.26 \%$. The next most performant variables are the HTT V2 and CMSTT masses and $\tau_{3} / \tau_{2}$. Considering pairs of variables, combining $\chi$ with b-tagging or groomed masses shows quite an improvement 

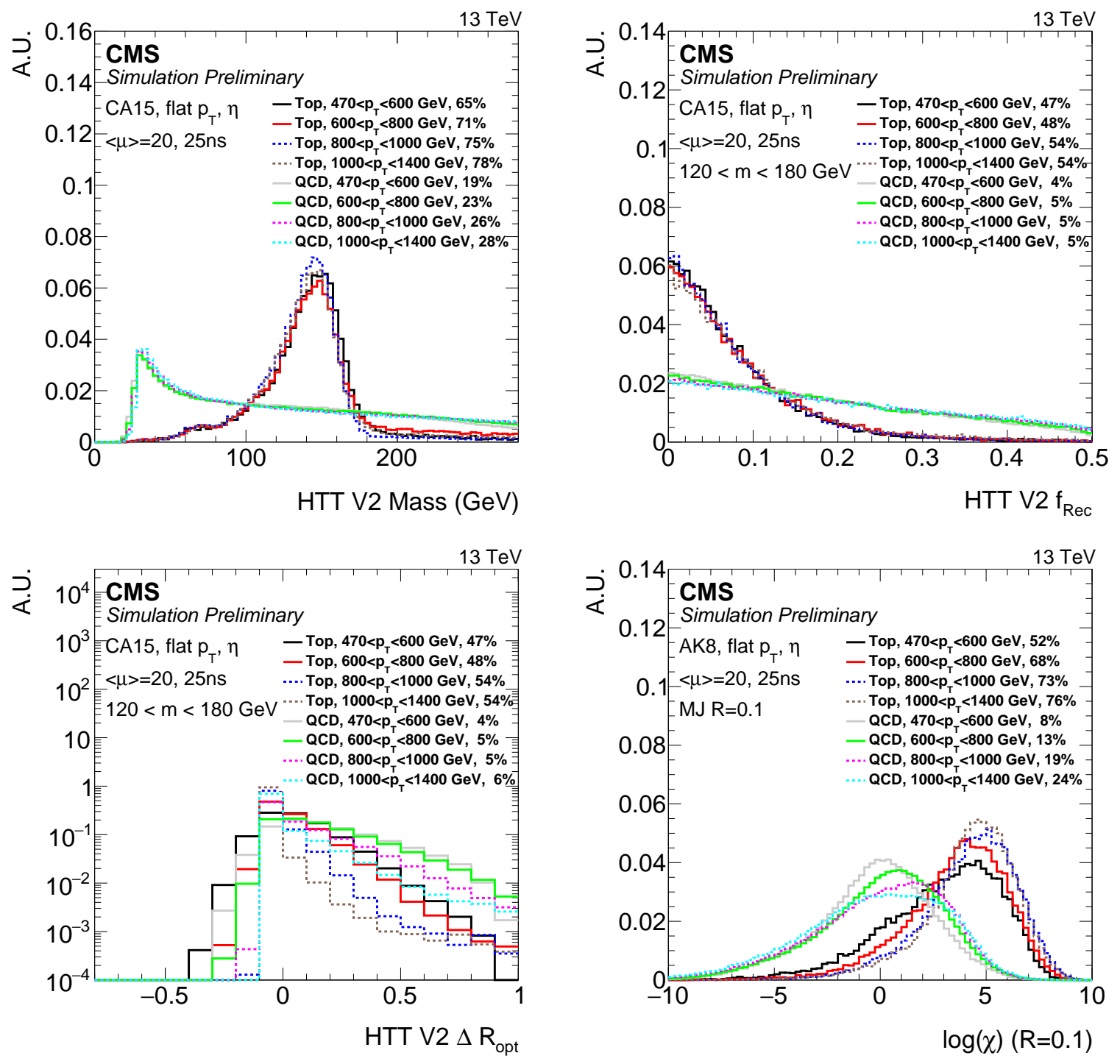

Figure 5.3.3: Distribution of HTT V2 mass (top left), HTT V2 $f_{\text {Rec }}$ (top right), HTT V2 $\Delta R_{\text {opt }}$ (bottom left), and $\log (\chi)$ (bottom right). The HTT V2 variables use CA15 jets as inputs, while the shower deconstruction variable uses AK8 jets. The percentages in the legends indicate the number of entries plotted in each histogram, with respect to the fiducial selection. Events were generated with an average of $\langle\mu\rangle=20$ pileup interactions and a 25-ns spacing between proton bunches [48]. 

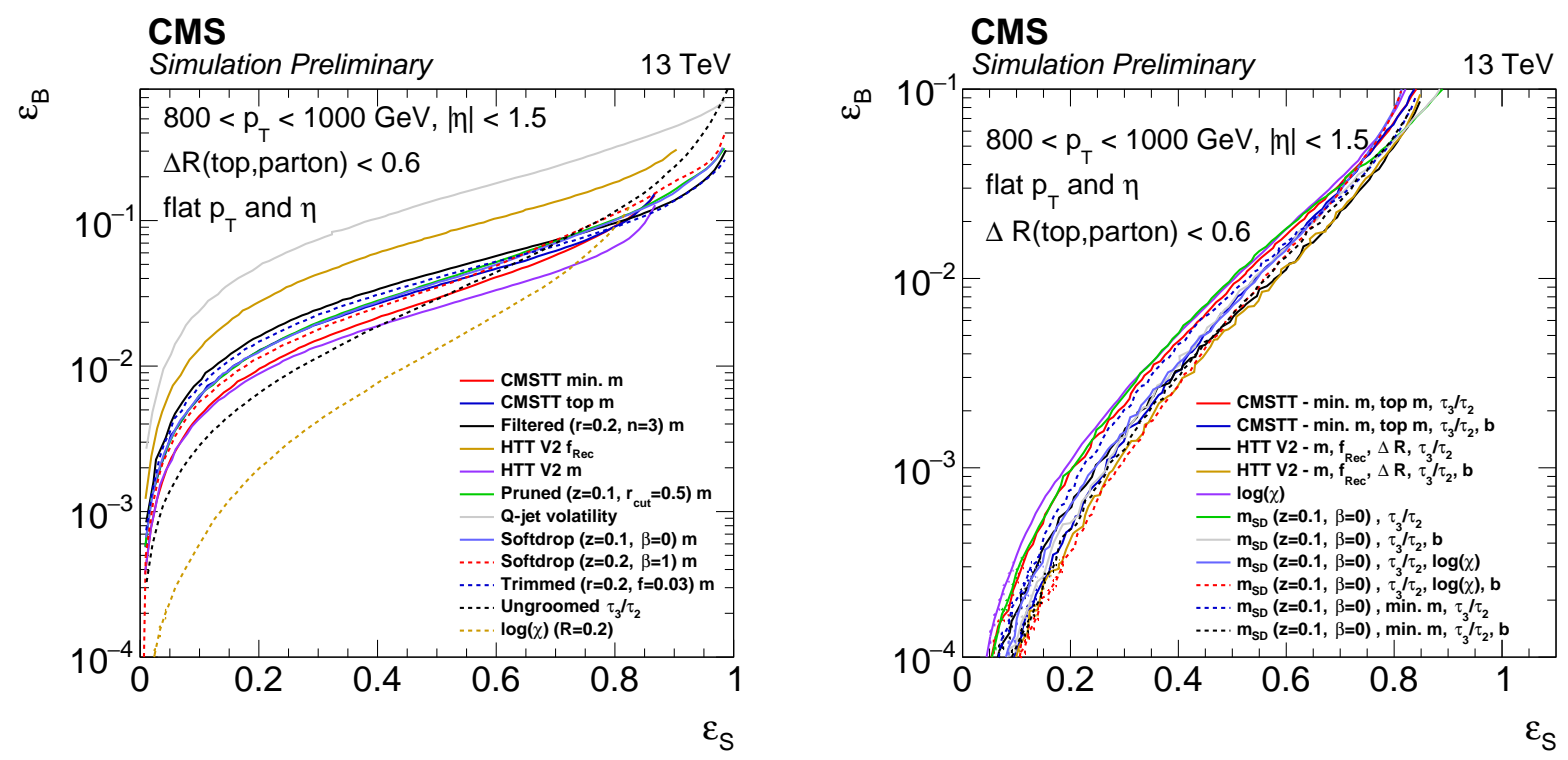

Figure 5.4.1: ROC curves: single-variable (left) and combined-variable (right). Each point on a given curve represents a cut-based selection window applied to the corresponding observable(s) [48].

(50-100\%) over using $\chi$ alone. Additionally, combining $\tau_{3} / \tau_{2}$ with a groomed mass variable provides good discrimination power.

After the $z$-score diagram is evaluated, ROC curves are produced for the most promising variable combinations (Fig. 5.4.1 right). Each point on a given curve corresponds to a selection window for each of the associated variables. Compared to the single variable ROC curves, the combined variables show a significant improvement in performance. Most of the combined-variable curves are similarly performant, with those including b-tagging achieving the best overall performance.

\subsection{Tagger Efficiency Kinematic Dependence}

In this section, the observables previously described are examined, comparing the performance of top tagging working points defined in Table 5.5. An optimal working point should provide high signal efficiency and background rejection across a wide range of jet $p_{T}$ and $|\eta|$ values, as well as maintain stable performance for large amounts of pileup activity in the 


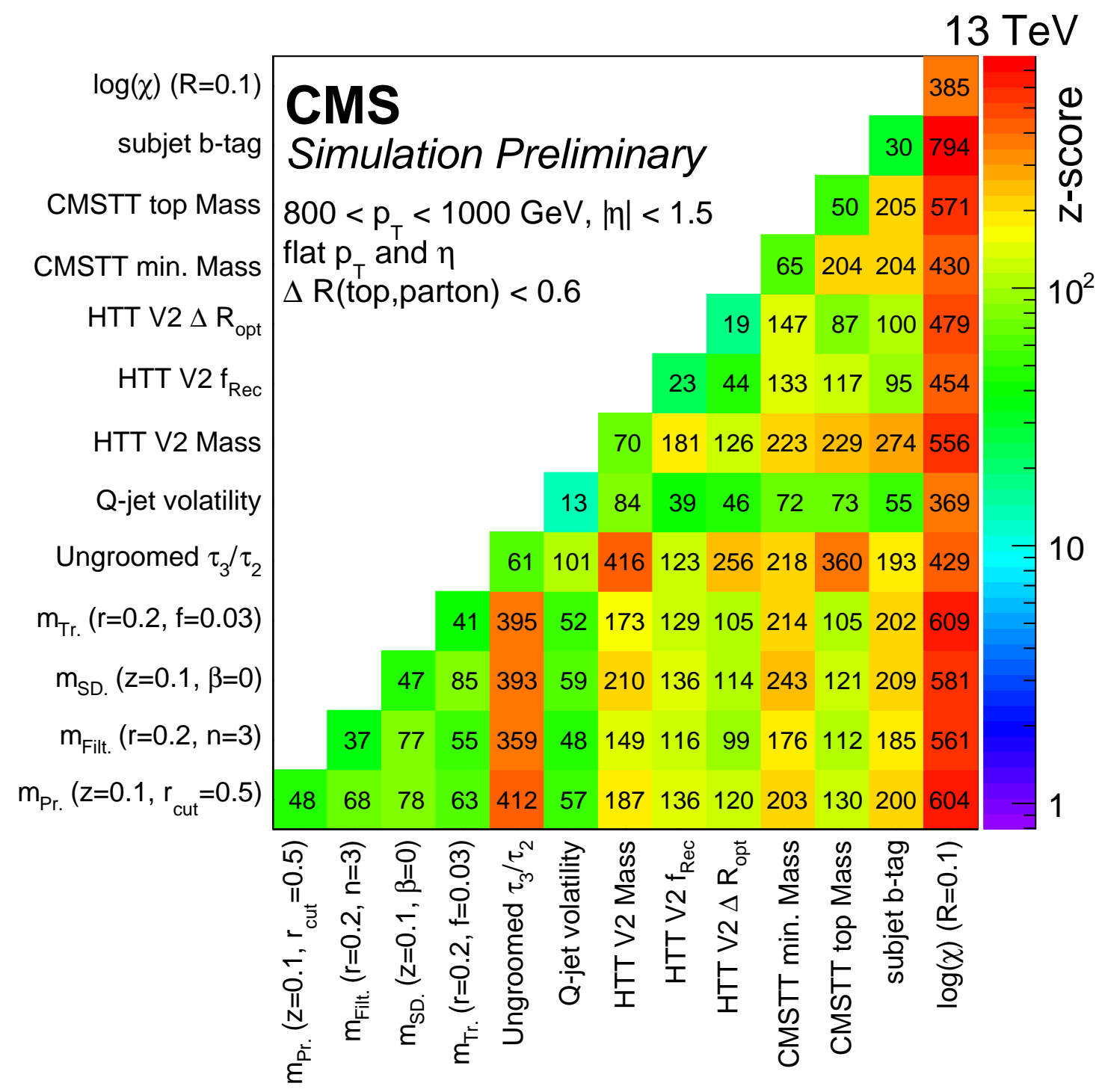

Figure 5.4.2: Combined-variable $z$-score diagram, where $z$-score is defined as $1 / \varepsilon_{B}$ when the signal efficiency is equal to $30 \%$. A projective likelihood estimator is used for the singlevariable entries on the diagonal, while a Boosted Decision Tree is used for the off-diagonal entries [48]. 
Substructure Algorithms Used for High- $p_{T}$ Working Points

\begin{tabular}{|c|c|c|c|c|}
\hline $110<M_{S D}<210 \mathrm{GeV}$ & & & $\tau_{3} / \tau_{2}<0.5$ & \\
\hline $110<M_{S D}<210 \mathrm{GeV}$ & & & $\tau_{3} / \tau_{2}<0.6$ & b-tag $>0.8$ \\
\hline $140<$ CMSTT Mass $<250 \mathrm{GeV}$ & & & $\tau_{3} / \tau_{2}<0.5$ & \\
\hline $140<$ CMSTT Mass $<250 \mathrm{GeV}$ & & & $\tau_{3} / \tau_{2}<0.6$ & $\mathrm{~b}-\operatorname{tag}>0.8$ \\
\hline $120<$ HTT V2 Mass $<180 \mathrm{GeV}$ & $f_{\operatorname{Rec}}<0.2$ & $-0.1<\Delta R_{o p t}<0.1$ & $\tau_{3} / \tau_{2}<0.6$ & \\
\hline $90<$ HTT V2 Mass $<170 \mathrm{GeV}$ & $f_{\text {Rec }}<0.2$ & $-0.2<\Delta R_{o p t}<0.2$ & $\tau_{3} / \tau_{2}<0.7$ & b-tag $>0.8$ \\
\hline $\begin{aligned} 90 & <M_{S D}<180 \mathrm{GeV} \\
70 & <M_{S D}<190 \mathrm{GeV}\end{aligned}$ & $\begin{array}{l}\log \left(\chi_{1}\right)>5.4 \\
\log \left(\chi_{1}\right)>4.6 \\
\log \left(\chi_{1}\right)>3.9\end{array}$ & & $\begin{array}{l}\tau_{3} / \tau_{2}<0.7 \\
\tau_{3} / \tau_{2}<0.7\end{array}$ & $\mathrm{~b}-\operatorname{tag}>0.6$ \\
\hline
\end{tabular}

Table 5.2: Summary of working points considered for studying their dependence on top quark $p_{T}$ and number of pileup vertices. The working points here correspond to a background efficiency of $0.3 \%$. The quantity $\chi_{1}$ refers to the shower deconstruction output using a microjet size of $R=0.1$ [48].

event. First, a working point is chosen to correspond to a background efficiency of $0.3 \%$, using the ROC curves previously shown in Fig. 5.4.1, without reweighting. The values come from a single bin in $p_{T}$, but the working point is then tested for stability across $p_{T}$ and number of pileup vertices.

Signal efficiency and mistag rate are shown in Fig. 5.5.1 as a function of the generator truth matched parton $p_{T}$ and the number of pileup vertices. The error bars represent the statistical uncertainty in each specific bin, due to a limited amount of simulated events. In all cases, the working points are chosen to correspond to a background tagging effiency of $0.3 \%$ in the inclusive sample. No merged top requirement is enforced in the signal sample to avoid an artificial increase of the top tagging efficiency.

A visible turn-on is observed as a function of the top quark transverse momentum, from 400 to $600 \mathrm{GeV}$. This is due to the decrease in angular distance between the top quark decay products approaching the threshold of the jet cone size. Above this transition, the efficiencies for the various algorithms are relatively flat as a function of jet $p_{T}$, with the exception of shower deconstruction, which has a much slower turn-on behavior. When combined with softdrop mass, subjet b-tagging, and $\tau_{3} / \tau_{2}$, shower deconstruction is found to have the highest tagging efficiency. However, jets tagged using shower deconstruction have a mistag rate that increases dramatically with $p_{T}$, which is not ideal for performing an analysis over a large $p_{T}$ 
range.

Based on the stable performance shown, softdrop combined with n-subjettiness has been recommended to be used as the primary strategy for top tagging at high $p_{T}$. This tagger combination is referred to as CMSTT version two for high $p_{T}$ (CMSTT V2H). To increase background rejection and signal efficiency stability, subjet b-tagging can also be added. The $Z^{\prime}$ search described in this dissertation uses an updated version of the CMSTT V2H, with working points tailored to PUPPI softdrop jets. 

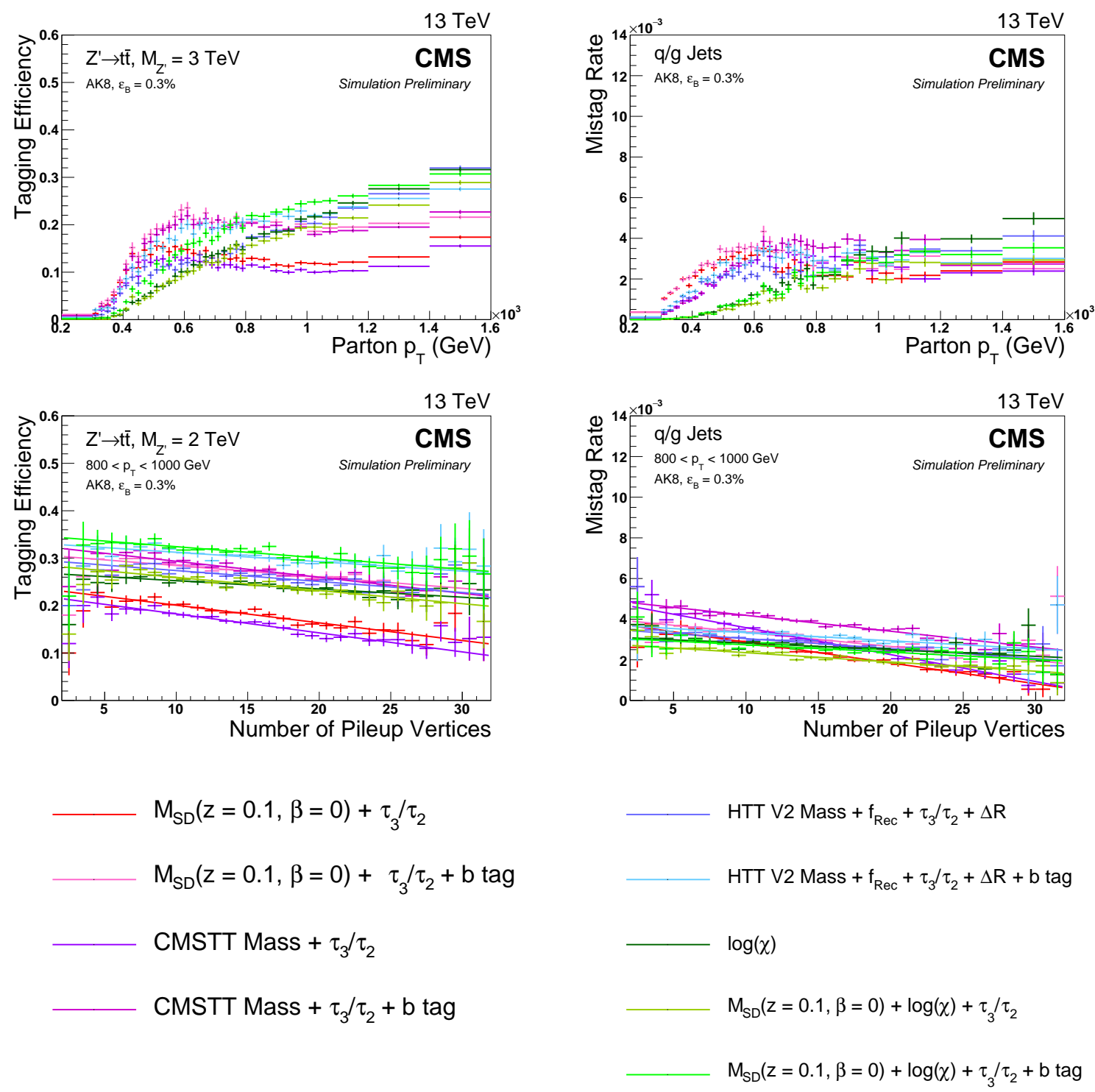

Figure 5.5.1: Top tagging efficiency for the high- $p_{T}$ working points listed in Table 5.5. The top plots show efficiency as a function of parton $p_{T}$, while the bottom ones show efficiency as a function of the number of pileup vertices. The plots in the first column are based on a $Z^{\prime} \rightarrow t \bar{t}$ sample with $M_{Z^{\prime}}=3 \mathrm{TeV}$ or $2 \mathrm{TeV}$, while those in the second column refer to QCD multijet production. The top right plot uses a flat parton $p_{T}$ distribution whereas a $p_{T}=300-470 \mathrm{GeV}$ QCD background sample is used for the bottom right one [48]. 


\section{Chapter 6}

\section{Analysis Selections}

A search is performed for heavy resonances in the top-antitop-quark pair $(t \bar{t})$ invariant mass spectrum $\left(m_{t \bar{t}}\right)$ to test for the presence of different new-physics models. As introduced in Section 2.2.2, the analysis tests for generic leptophobic topcolor models, with the associated resonance labeled as $Z^{\prime}$, as well as more specific models, such as Randall-Sundrum KaluzaKlein gluon production.

Previous searches have set limits for such resonances, with masses below $900 \mathrm{GeV}$, by the CDF and D0 experiments at the Tevatron [57, 58]. At the LHC, the CMS and ATLAS experiments have continued to set limits for heavy resonances decaying to $t \bar{t}$. To do so, the experiments have used data from proton-proton collisions at center of mass energies of 7 and 8 TeV to set increasingly strict limits on resonances above $1 \mathrm{TeV}$ [59, 60, 61, 62, 63, 64, 65, 66]. Most recently, CMS and ATLAS have released $t \bar{t}$ resonance limits, using 2.6 and $3.2 \mathrm{fb}^{-1}$ of data, respectively, from the first year (2015) of $13 \mathrm{TeV}$ collisions at the LHC [67, 68]. In 2016, the CMS experiment recorded an order of magnitude larger dataset $\left(36 \mathrm{fb}^{-1}\right)$, thus enabling a more sensitive search to be performed. The work presented here focuses on an on-going 2016 CMS search, combined with the main results of the 2015 CMS search.

$Z^{\prime} \rightarrow t \bar{t}$ analyses are categorized by the decay modes of the top-antitop pair, with each top decaying into a b quark and a $\mathrm{W}$ boson. The $\mathrm{W}$ subsequently decays into a lepton and 
a neutrino or into two quarks that each produce a jet of hadrons. The three top-pair decay possibilities are labeled as all-hadronic (both W's decay into quarks), dileptonic (both W's decay into leptons), or semileptonic (one W decays into leptons and the other decays into quarks).

This analysis considers the all-hadronic channel. Due to the high mass of the resonance search region, each top quark is highly boosted, and its decay products are fully merged into a single jet. For this decay topology, special top tagging algorithms, built on identifying substructure within single jets, are used. The tagger used in this analysis is optimized for separating tops with large boost $\left(p_{T}>400\right)$ from QCD jets. Known as the CMS Top Tagger (V2), it is described in detail in Chapter 5. This algorithm relies on softdrop grooming, the n-subjettiness jet shape variable, and subjet b-tagging algorithms to identify top jets. For the first time in a $Z^{\prime} \rightarrow t \bar{t}$ search, this analysis utilizes PUPPI before calculating top tagging variables [37]. PUPPI greatly reduces the effects of pileup on top tagging performance.

The dominant background for this analysis, in which the selection consists of dijet events, is multijet events originating from nontop QCD interactions (nontop multijet, i.e., NTMJ). This background is estimated using a top-tag mistag rate measured using a control region in data. This mistag rate is then applied to data events to estimate the NTMJ background in the signal region. In the most sensitive signal regions, the dominant background is Standard Model $t \bar{t}$ production, which is estimated using simulated events. After the background model is validated, the analysis looks for deviations from the expected SM $t \bar{t}$ invariant mass spectrum, testing for the presence of several different signal hypotheses, including narrow, wide, and extra wide $\left(1 \%, 10 \%\right.$, and $30 \%$ widths, respectively) $Z^{\prime}$ samples, and RandallSundrum Kaluza-Klein gluon models. 


\subsection{Samples}

\subsubsection{Data Samples}

The data used in this analysis were collected in 2016 during Run 2 of the LHC, for which the center-of-mass energy was $\sqrt{s}=13 \mathrm{TeV}$, corresponding to an integrated luminosity of $36 \mathrm{fb}^{-1}$.

\subsubsection{Simulated Background Samples}

Samples of simulated events are used to estimate the background from SM continuum $t \bar{t}$ events. These samples were generated with POWHEG interfaced to PYTHIA8. To determine the expected number of events, a cross section of $\sigma_{t \bar{t}}=831.76 \mathrm{pb}$ is used for normalization [69]. The $t \bar{t}$ samples used in this analysis are inclusive in the top decay products.

A data-driven method is used to estimate the non-top multijet (NTMJ) background in the analysis, while simulated QCD Monte Carlo events are used to test the analysis methods and to test the closure of the NTMJ background estimate technique. Both QCD Monte Carlo simulated with MADGRAPH and showered with PYTHIA8 and QCD Monte Carlo simulated and showered with PYTHIA8 are considered. While both samples are used for the closure tests and kinematic plots in Appendix B. Figs. 6.2.7 - 6.2.9 are plotted using the latter QCD sample, as it is found to better model the data.

\subsubsection{Simulated Signal Samples}

Table 6.1 lists $Z^{\prime}$ samples with widths set to $1 \%$ of the mass of the $Z^{\prime}$, Table 6.2 lists $Z^{\prime}$ samples with widths set to $10 \%$ of the mass of the $Z^{\prime}$, Table 6.3 lists $Z^{\prime}$ samples with widths set to $30 \%$ of the mass of the $Z^{\prime}$, and Table 6.4 lists Randall-Sundrum Gluon samples with widths approximately $17 \%$ of the mass of the RS Gluon. Samples were generated centrally in CMS as part of the "RunIISummer16" campaign, using CMS software (CMSSW) version 8. Mass points between 1 and $5 \mathrm{TeV}$, in increments of $500 \mathrm{GeV}$, are used. 


\begin{tabular}{llr} 
Sample & Events & $\sigma(\mathbf{p b}), \mathbf{N L O}$ \\
\hline$Z^{\prime} 1 \mathrm{TeV}$ mass, $1 \%$ width & 103785 & 4.505 \\
$Z^{\prime} 1.25 \mathrm{TeV}$ mass, $1 \%$ width & 102833 & 1.809 \\
$Z^{\prime} 1.5 \mathrm{TeV}$ mass, $1 \%$ width & 99690 & 0.814 \\
$Z^{\prime} 2.5 \mathrm{TeV}$ mass, $1 \%$ width & 100288 & 0.0617 \\
$Z^{\prime} 3 \mathrm{TeV}$ mass, $1 \%$ width & 91004 & 0.0206 \\
$Z^{\prime} 3.5 \mathrm{TeV}$ mass, $1 \%$ width & 91004 & 0.00735 \\
$Z^{\prime} 4 \mathrm{TeV}$ mass, $1 \%$ width & 107914 & 0.00276 \\
$Z^{\prime} 4.5 \mathrm{TeV}$ mass, $1 \%$ width & 100306 & 0.00109 \\
$Z^{\prime} 5 \mathrm{TeV}$ mass, $1 \%$ width & 112042 & 0.000458
\end{tabular}

Table 6.1: Signal Monte Carlo samples used in the analysis, for the $Z^{\prime}$ samples of $1 \%$ widths.

\begin{tabular}{llr} 
Sample & Events & $\sigma(\mathbf{p b}), \mathbf{N L O}$ \\
\hline$Z^{\prime} 1 \mathrm{TeV}$ mass, $10 \%$ width & 101056 & 44.853 \\
$Z^{\prime} 1.25 \mathrm{TeV}$ mass, $10 \%$ width & 96845 & 18.374 \\
$Z^{\prime} 1.5 \mathrm{TeV}$ mass, $10 \%$ width & 111108 & 8.476 \\
$Z^{\prime} 2 \mathrm{TeV}$ mass, $10 \%$ width & 104119 & 2.262 \\
$Z^{\prime} 2.5 \mathrm{TeV}$ mass, $10 \%$ width & 96077 & 0.734 \\
$Z^{\prime} 3 \mathrm{TeV}$ mass, 10\% width & 189164 & 0.273 \\
$Z^{\prime} 3.5 \mathrm{TeV}$ mass, $10 \%$ width & 101022 & 0.113 \\
$Z^{\prime} 4 \mathrm{TeV}$ mass, $10 \%$ width & 102411 & 0.0516 \\
$Z^{\prime} 4.5 \mathrm{TeV}$ mass, $10 \%$ width & 84504 & 0.0259 \\
$Z^{\prime} 5 \mathrm{TeV}$ mass, $10 \%$ width & 107156 & 0.0143
\end{tabular}

Table 6.2: Signal Monte Carlo samples used in the analysis, for the $Z^{\prime}$ samples of $10 \%$ widths.

\begin{tabular}{llr} 
Sample & Events & $\sigma(\mathbf{p b})$, NLO \\
\hline$Z^{\prime} 1 \mathrm{TeV}$ mass, 30\% width & 79477 & 129.361 \\
$Z^{\prime} 2 \mathrm{TeV}$ mass, 30\% width & 114009 & 7.742 \\
$Z^{\prime} 4 \mathrm{TeV}$ mass, 30\% width & 88039 & 0.289 \\
$Z^{\prime} 5 \mathrm{TeV}$ mass, 30\% width & 91038 & 0.0996
\end{tabular}

Table 6.3: Signal Monte Carlo samples used in the analysis, for the $Z^{\prime}$ samples of $30 \%$ widths. 


\begin{tabular}{llr} 
Sample & Events & $\sigma(\mathbf{p b}), \mathbf{L O} \times \mathbf{1 . 3}$ \\
\hline RSG $1 \mathrm{TeV}, \sim 17 \%$ width & 98560 & $20.05 \times 1.3$ \\
RSG $1.25 \mathrm{TeV}, \sim 17 \%$ width & 100000 & $7.92 \times 1.3$ \\
RSG $1.5 \mathrm{TeV}, \sim 17 \%$ width & 100000 & $3.519 \times 1.3$ \\
RSG 2 TeV, $\sim 17 \%$ width & 100000 & $0.9528 \times 1.3$ \\
RSG $2.5 \mathrm{TeV}, \sim 17 \%$ width & 100000 & $0.3136 \times 1.3$ \\
RSG 3 TeV, $17 \%$ width & 99755 & $0.1289 \times 1.3$ \\
RSG 3.5 TeV, 17\% width & 99508 & $0.05452 \times 1.3$ \\
RSG 4 TeV,$\sim 17 \%$ width & 99136 & $0.02807 \times 1.3$
\end{tabular}

Table 6.4: Signal Monte Carlo samples used in the analysis, for the Randall-Sundrum Gluon samples of $\sim 17 \%$ widths.

\subsubsection{Pileup Reweighting}

The samples used are simulated for the scenario with 25 ns bunch spacing and a pileup scenario which approximates the 2016 data-taking conditions. The simulated signal and background events are reweighted to accurately model the pileup conditions in data, using a minimum bias cross section of $69.2 \mathrm{mb}( \pm 4.6 \%)$ (Fig. 6.1.1).
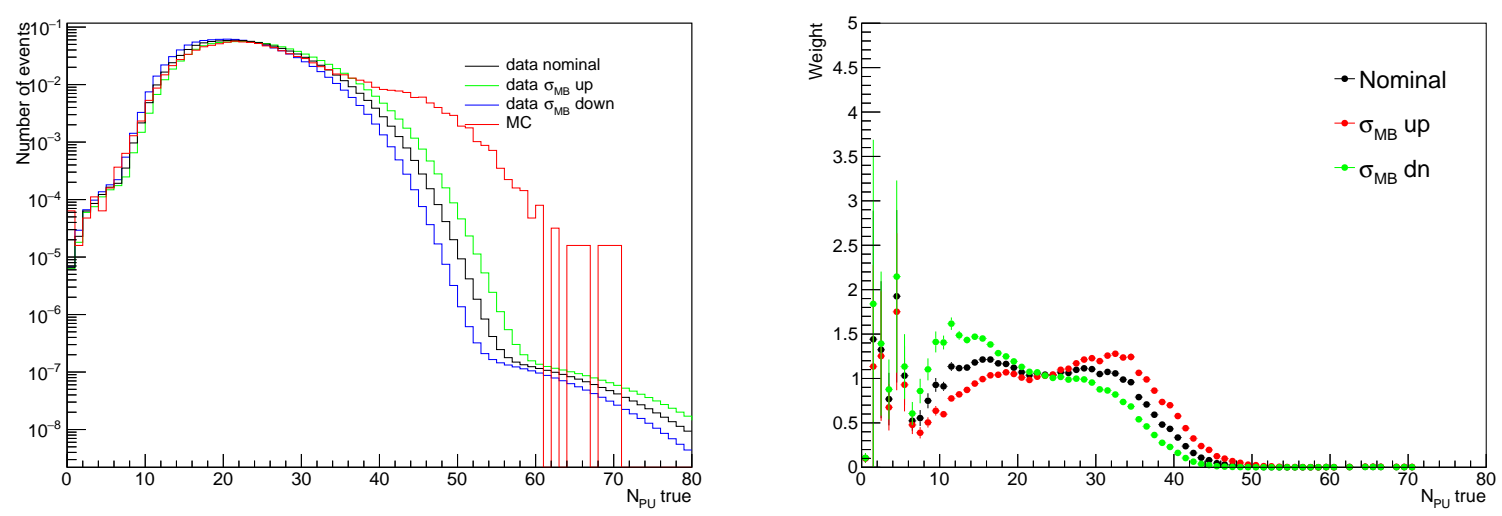

Figure 6.1.1: (left) Pileup distribution in data and Monte Carlo. (right) PU weight applied to Monte Carlo. 


\subsection{Event Selection}

\subsubsection{Trigger Selection}

In this analysis, the OR of the following jet HT, single jet, and groomed jet triggers is used:

- PFHT800

- PFHT900

- PFHT700TrimMass50

- AK8PFJet450

- PFJet360TrimMass30

In order to determine the best set of triggers for the analysis, trigger efficiency is considered as a function of $H_{T}$ (Fig. 6.2.1), the sensitive variable dijet mass (Fig. 6.2.2), and both $H_{T}$ and PUPPI softdrop mass (6.2.3). The $H_{T}$ of an event is defined as the scalar sum of the $p_{T}$ of every AK4CHS jet $\left(p_{T}^{\mathrm{AK} 4}>30,\left|\eta^{\mathrm{AK} 4}\right|<3.0\right)$ in the event. Dijet mass is defined as the invariant mass of the two $p_{T}$-leading jets. The efficiency is measured as the number of events that pass the numerator selection, trigger of interest OR Mu50 OR IsoMu24, divided by the number of events that pass the denominator selection, Mu50 OR IsoMu24. A preselection requirement, two AK8 jets with $|\Delta \phi>2.1|$ and $p_{T}>400 \mathrm{GeV}$, is applied to all plots. All three figures include efficiency plots with an additional preselection requirement that at least one jet have its mass in the top tag window $\left(105 \mathrm{GeV}<m_{\mathrm{PUPPI}, \mathrm{SD}}<210 \mathrm{GeV}\right)$, as this requirement is made in both the signal region and the background estimate sideband.

Comparing the trigger combinations in Fig. 6.2.1, it is evident that the combination of the triggers listed above is the most performant. As shown in the top left plot, a cut of $H_{T}>950 \mathrm{GeV}$ allows the analysis to have $>99.8 \%$ efficiency. The left plot in Fig. 6.2.2, in which this $950 \mathrm{GeV} H_{T}$ cut has been applied, shows that in the dijet mass region of interest $\left(M_{j j}>1000 \mathrm{GeV}\right)$, the combination of triggers and preselection cuts yields a $>99.9 \%$ trigger 
efficiency. Additionally, considering the right plot in Fig. 6.2.3, it is evident that for events with $H_{T}>950 \mathrm{GeV}$, the analysis trigger selection is highly performant, and the efficiency as a function of $H_{T}$ does not depend on jet mass.
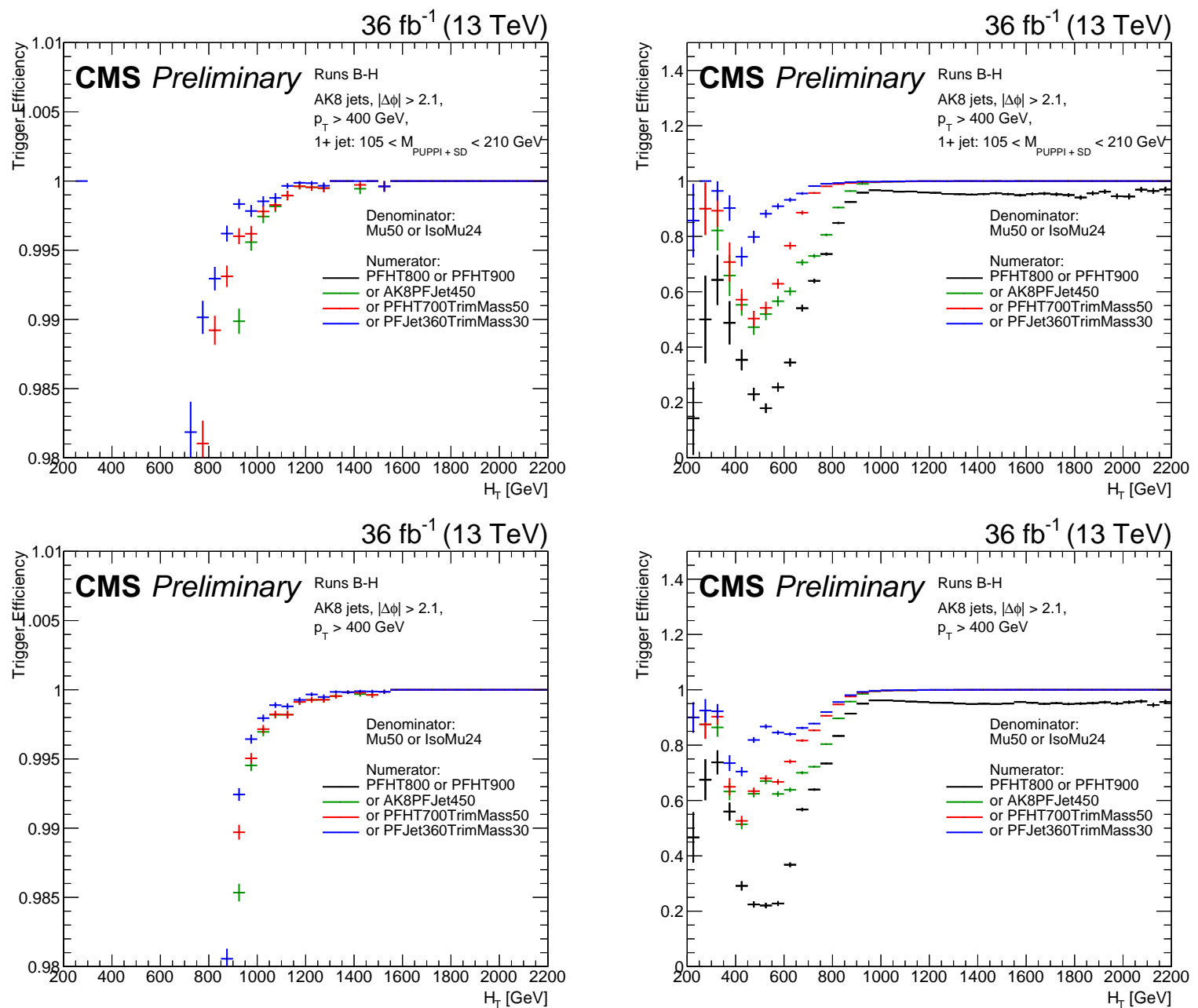

Figure 6.2.1: Trigger efficiency as a function of $H_{T}$. Plots in the right column use a y-axis range from 0.0 to 1.5, while plots in the left column use a y-axis range from 0.98 to 1.01. All events are required to have two AK8 jets with $|\Delta \phi>2.1|$ and $p_{T}>400 \mathrm{GeV}$. The plots in row 1 include an additional requirement that each event contain at least one jet with its mass in the top mass window. The legend describes the different combinations of jet HT, single jet, and groomed jet triggers compared. The triggers used for each curve correspond to the associated entry in the legend, combined with all preceding entries. For example, the red curve indicates the trigger efficiency of the OR combination of the PFHT800, PFHT900, AK8PFJet450, PFHT700TrimMass50, and denominator triggers. Efficiencies are plotted with an OR of the Mu50 and IsoMu24 triggers in the denominator. 

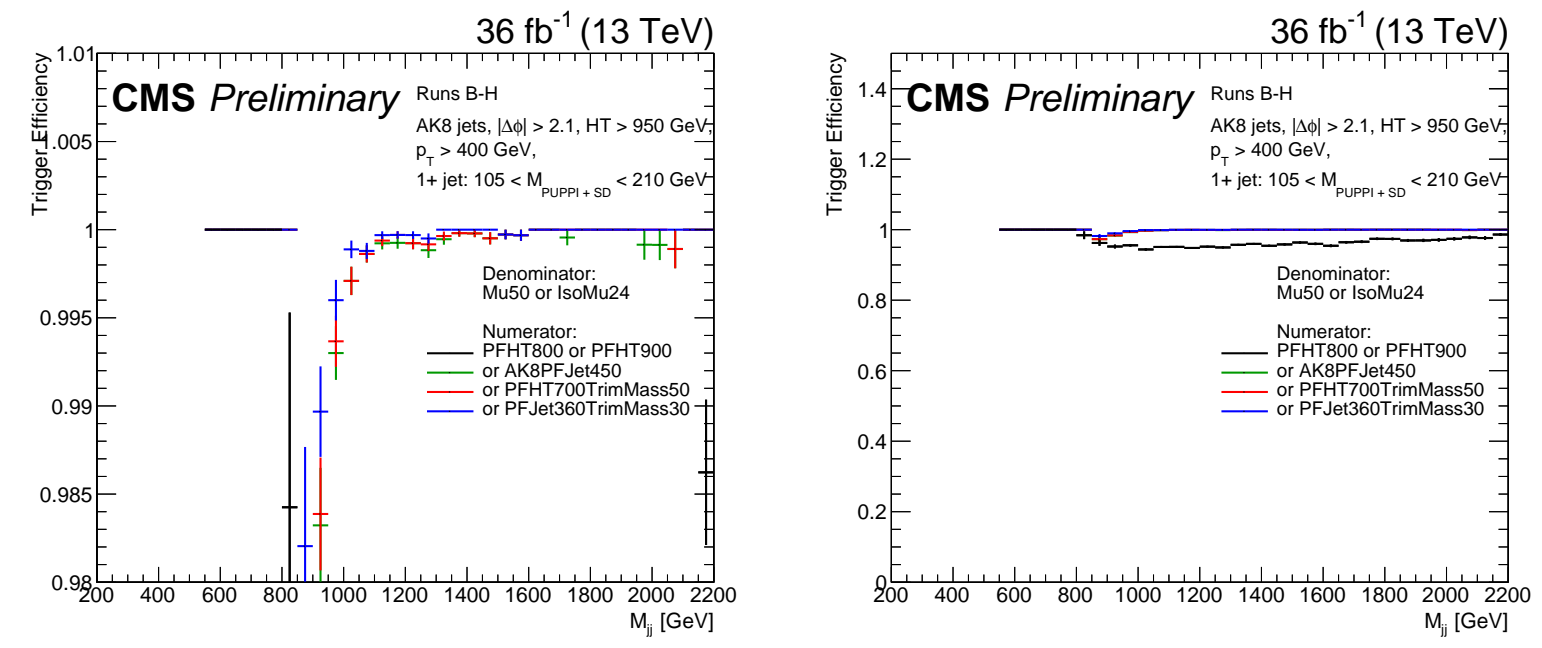

Figure 6.2.2: Trigger efficiency as a function of dijet mass, the sensitive variable of this analysis. The right plot has a y-axis range from 0.0 to 1.5 , while the left plot has a y-axis range from 0.98 to 1.01. All events are required to have two AK8 jets with $|\Delta \phi>2.1|$ and $p_{T}>400 \mathrm{GeV}$, as well as at least one jet with its mass in the top mass window. The legend describes the different combinations of jet HT, single jet, and groomed jet triggers compared. The triggers used for each curve correspond to the associated entry in the legend, combined with all preceding entries. For example, the red curve indicates the trigger efficiency of the OR combination of the PFHT800, PFHT900, AK8PFJet450, PFHT700TrimMass50, and denominator triggers. Efficiencies are plotted with an OR of the Mu50 and IsoMu24 triggers in the denominator. 

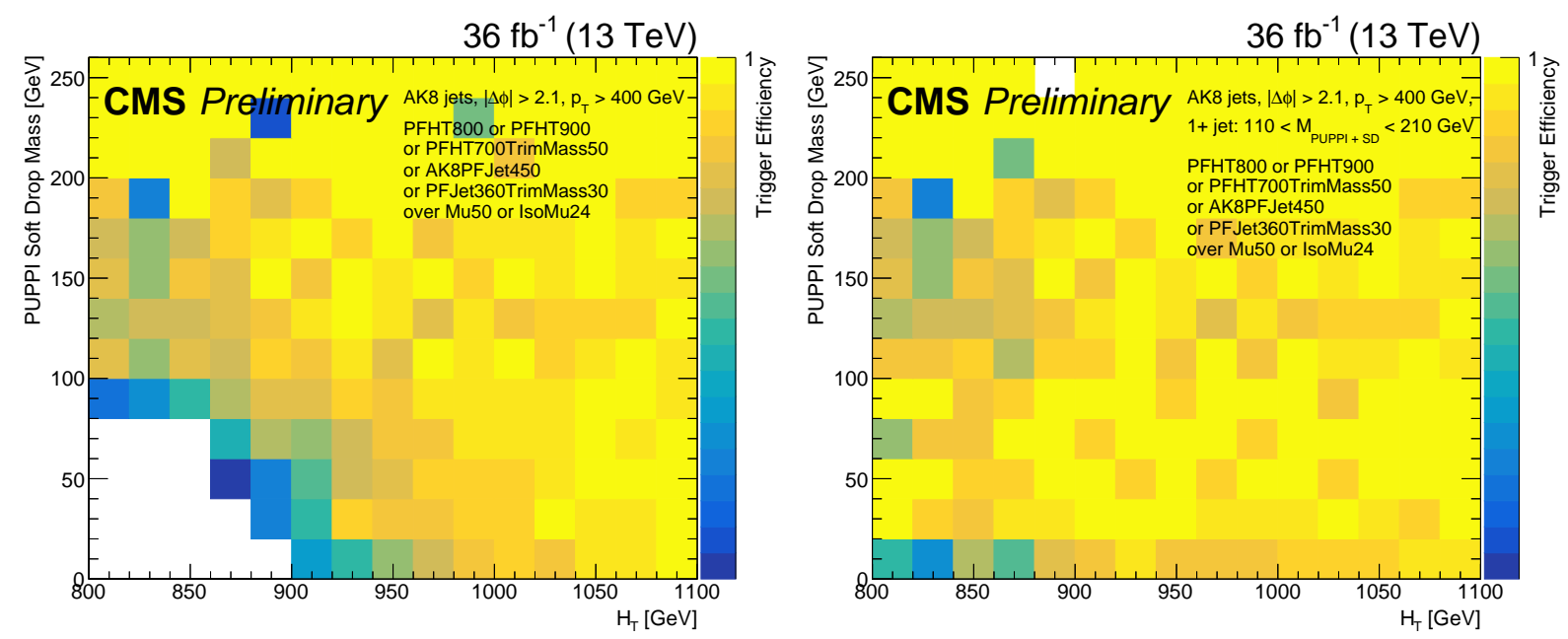

Figure 6.2.3: Trigger efficiency as a function of $H_{T}$ (x-axis) and PUPPI softdrop mass (yaxis). Plots have a z-axis range from 0.98 to 1.0. All events are required to have two AK8 jets with $|\Delta \phi>2.1|$ and $p_{T}>400 \mathrm{GeV}$. The plot on the right includes an additional requirement that each event contains at least one jet with its mass in the top mass window. Efficiencies are plotted for the full analysis trigger selection, with an OR of the Mu50 and IsoMu24 triggers in the denominator.

\subsubsection{Vertex Selection and Pileup Mitigation}

Primary vertices are reconstructed using a deterministic annealing filtering algorithm [70]. The leading primary vertex of the event is defined as the primary vertex with the largest squared sum of transverse momenta of clustered physics objects.

Events are required to have a good primary vertex that is "not fake" (i.e. at least one associated track), with $\left|z_{\text {Primary Vertex }}\right|<24 \mathrm{~cm}, N_{\text {DOF }}>4,|\rho|<2 \mathrm{~cm}$.

Charged hadrons associated with subleading primary vertices are removed from further consideration. This is referred to as "charged hadron subtraction" (CHS). In order to mitigate the effects of pileup on the jet mass and substructure selection (see below), PUPPI is used. This is more resilient to pileup than CHS for these observables. 


\subsubsection{Jet Reconstruction}

Jets are clustered using the anti- $k_{T} \mathrm{R}=0.8$ algorithm [36] with both CHS and PUPPI inputs. In this analysis, jets are formed out of particle flow $(\mathrm{PF})$ constituents [33]. Jets constructed with CHS are referred to as "PFCHS". Jets constructed with PUPPI are referred to as "PFPUPPI". AK8CHS jets are used for kinematic variables such as $p_{T}$ and dijet mass. AK8PUPPI jets are used only for top tagging and subjet b-tagging. An AK8PUPPI jet is matched to each AK8CHS jet by finding the closest AK8PUPPI jet which also satisfies $\Delta R(\mathrm{CHS}, \mathrm{PUPPI})<1.0$.

Simulated jets are corrected to better match the data. The corrections derived for AK8CHS jets are applied to the AK8CHS four-vector. This is done before any kinematic cuts are made. Corrections derived for AK8PUPPI jets are applied to the AK8PUPPI fourvector.

Three methods of correcting the AK8PUPPI groomed softdrop jet mass were investigated:

1. Apply AK8PUPPI corrections also to the groomed jet mass

2. Apply AK4PUPPI corrections to the individual softdrop subjets before calculating the groomed jet mass (the pairwise subjet mass)

3. Apply a specific jet mass correction developed for $\mathrm{W}$-tagging

The W-tagging based correction (correction method 3) is found to induce a shift in the top tagging mass peak as a function of $p_{T}$ and is therefore not used. Neither method 1 nor method 2 results in a significant shift in the top mass peak location with increasing $p_{T}$. Little difference between correction methods 1 and 2 is found - therefore method 1 is chosen for simplicity (Fig. 6.2.4).

Differing jet energy resolutions between simulated events and data events are taken into account. The recommended $\eta$-dependent smearing is applied to simulated jets (Spring16 25nsV10) before requiring any kinematic selection on the jets. Additionally, the groomed jet mass is smeared by the same value used for the jet momentum. 

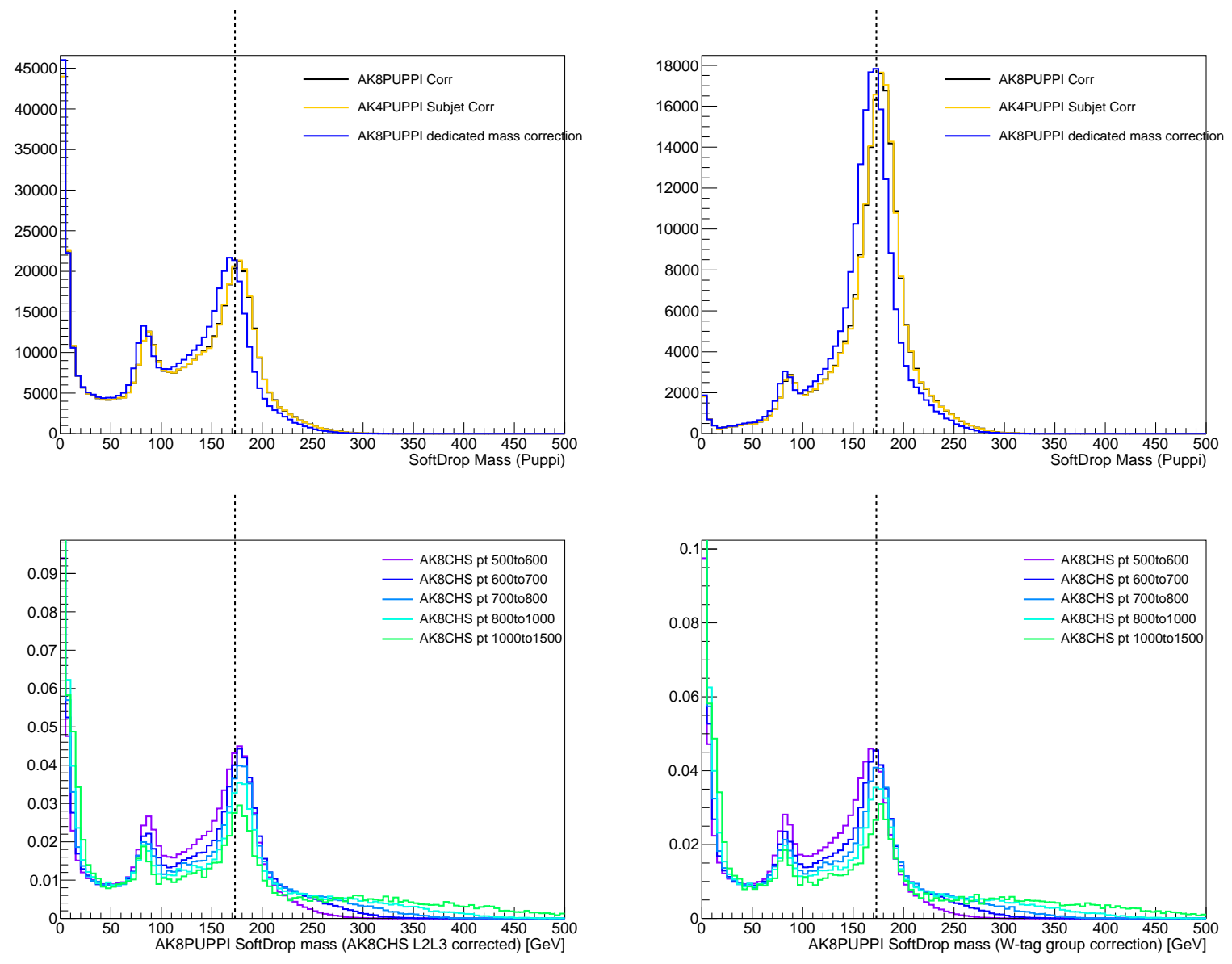

Figure 6.2.4: (top left) PUPPI softdrop mass in a $t \bar{t}$ MC sample comparing different mass correction methods. (top right) PUPPI softdrop mass after applying a $\tau_{32}$ cut in a $t \bar{t} \mathrm{MC}$ sample comparing different mass correction methods. (bottom left) PUPPI softdrop mass corrected with AK8PUPPI JEC in different $p_{T}$ regions. (bottom right) PUPPI softdrop mass corrected with the dedicated mass correction developed for $\mathrm{W}$-tagging in different $p_{T}$ regions. These plots show that corrections based on the $\mathrm{W}$ mass are inappropriate for the top quark tagger. Not much difference can be seen in mass when correcting the entire jet or each subjet. Therefore, for simplicity, the first option is used. 
The following "Jet ID" criteria are applied in order to remove jets originating from detector noise:

- PFJet Neutral Hadron Fraction $<0.99$

- PFJet Neutral EM Fraction $<0.99$

- PFJet Number of Constituents $>1$

- PF Jet Charged Hadron Fraction $>0$

- PFJet Charged Multiplicity $>0$

- PFJet Charged EM Fraction $<0.99$

\subsubsection{Top $p_{T}$ reweighting}

As measured by the TOP PAG (Physics Analysis Group), the ratio of the top $p_{T}$ in data with respect to the theory prediction exhibits a slope, and therefore a correction factor has been developed [71, 72]. The correction scale factor is given by $S F\left(p_{T}^{G E N}\right)=e^{0.0615-0.0005 p_{T}}$ and therefore the overall event weight becomes $W=\sqrt{S F(t) S F(\bar{t})}$. A comparison of the jet $p_{T}$ distribution with and without top $p_{T}$ reweighting is shown in Figure 6.2.5. Comparisons of event and jet kinematic variables is shown in Appendix C. The main analysis is performed with top $p_{T}$ reweighting applied to $t \bar{t} \mathrm{MC}$. In order to measure the corresponding systematic uncertainty, the analysis is performed again, this time without top $p_{T}$ reweighting.

\subsubsection{Event Preselection}

The following preselection is applied.

- The event is required to have at least two AK8CHS jets which satisfy:

$$
-p_{T}>400 \mathrm{GeV}
$$



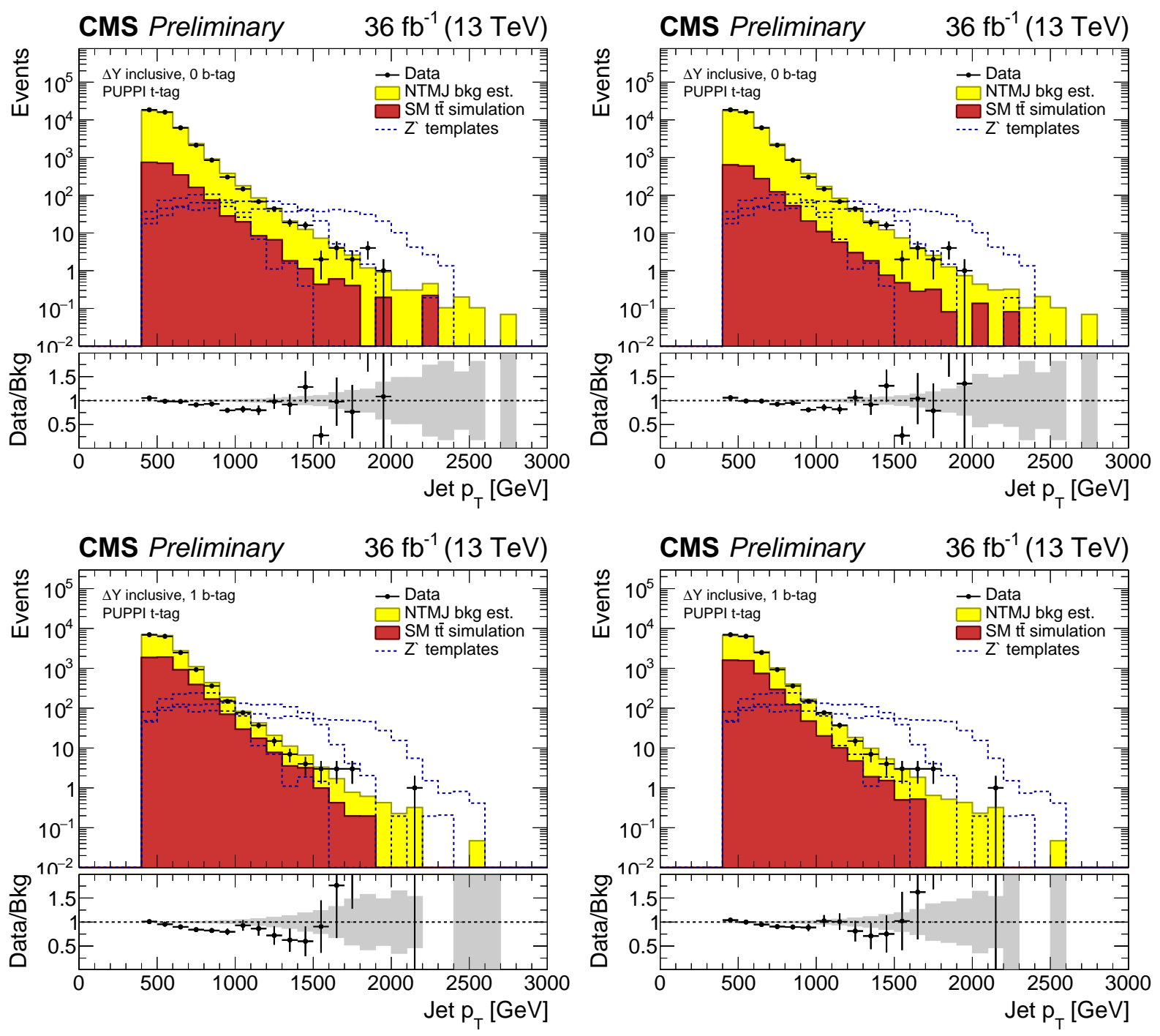

Figure 6.2.5: Jet $p_{T}$ distribution in data compared to the data-driven NTMJ (described in section 7 ) and $t \bar{t} \mathrm{MC}$ backgrounds. (top left) $0 \mathrm{~b}$-tag with no top $p_{T}$ reweighting, (top right) 0 b-tag with top $p_{T}$ reweighting, (bottom left) 1 b-tag with no top $p_{T}$ reweighting, (bottom right) 1 b-tag with top $p_{T}$ reweighting. The plots with top $p_{T}$ reweighting applied show a somewhat better data/MC agreement. 
$-\mathrm{HT}>950 \mathrm{GeV}$

$-|y|<2.4$

- Loose jet ID (section 6.2.3)

- To select the back-to-back topology, the leading two jets are required to be separated by the azimuthal angle $\Delta \phi>2.1$.

- Standard event noise cleaning filters

\subsubsection{Top Tagging Algorithms}

The products of hadronically decaying top quarks can fall within a single jet if the top quark is highly boosted relative to its mass. Special tools, known as top tagging algorithms, are designed to identify these boosted decay topologies, as previously discussed in Chapter 5 .

\section{CMS Top Tagger Version Two}

This algorithm uses a combination of substructure techniques for tagging top jets. For highly boosted tops $\left(p_{T}>400 \mathrm{GeV}\right)$, the CMS top tagger version two for high $p_{T}(\mathrm{CMSTT}$ V2H) is used, which takes anti- $k_{T} \mathrm{R}=0.8 \mathrm{PUPPI}$ jets as inputs [48. The input AK8 jets are hereby referred to as the "hard jets." As explained in Chapter 5, both n-subjettiness and jet softdrop mass are used to tag top jets. The subjet b-tagging definition is separated from the top tagging definition in order to later divide event categories based on the number of identified subjet b-tags. In this analysis, the top tagging variables $\tau_{3} / \tau_{2}$ and $m_{S D}$ are calculated from PUPPI inputs. The PUPPI-based top tagging algorithm demonstrates relatively stable tagging efficiency for all pileup conditions observed in 2016 (Fig. 6.2.6).

Figure 6.2.7 shows a comparison of the relevant CMSTT quantities in data, simulated $t \bar{t}$, and simulated QCD events. The discrimination power of each observable can be seen - each variable is shown with the requirements already placed on the other two quantities. In this way, one can ascertain the independent discrimination power offered by each variable. Some 

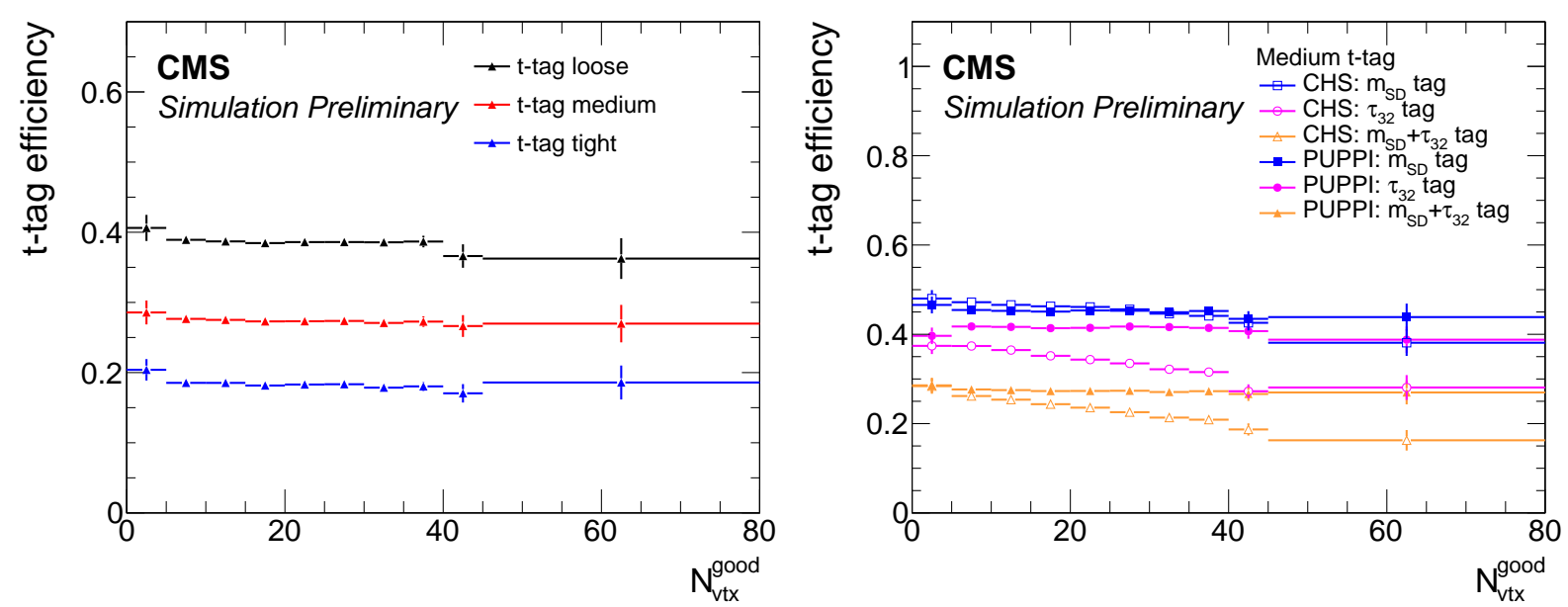

Figure 6.2.6: (left) PUPPI top tag efficiency for 3 different working points (right) top tag efficiency for CHS and PUPPI based variables. Three tag definitions are considered: $m_{S D}$ only, $\tau_{32}$ only, $m_{S D}+\tau_{32}$. The pileup dependence of CHS is still significant in the pileup regime of interest, whereas the dependence of PUPPI is flat. Therefore, the PUPPI-based variables for substructure tagging are used.

disagreement is observed due to the lack of scale factors available for cutting on a single top tagging variable. In these plots, there is a top tag SF applied to the opposite top tagged jet, but no SF is applied to the N-1 tagged jet. The application of softdrop jet mass resolution scale factors, as yet unmeasured for top jets, might also improve the agreement.

\subsubsection{Subjet b-tagging}

Subjet b-tagging is used to categorize events (Sec. 6.2.8). The standard CMS b quark ID combined secondary vertex (CSVv2) algorithm [53] (pfCombinedInclusiveSecondaryVertexV2BJetTags) is applied to the subjets found using the softdrop algorithm running on AK8PUPPI jets, as described above. A subjet is considered to be b-tagged if the CSV discriminant is greater than 0.8484 , corresponding to the medium-efficiency working point (CSVv2M). The loose working point was also considered but was found to be suboptimal (see Section 6.2.8). There is no official tight subjet b-tagging working point.

The subjet b-tag rate differs in data and in simulation and therefore subjet b-tagging 

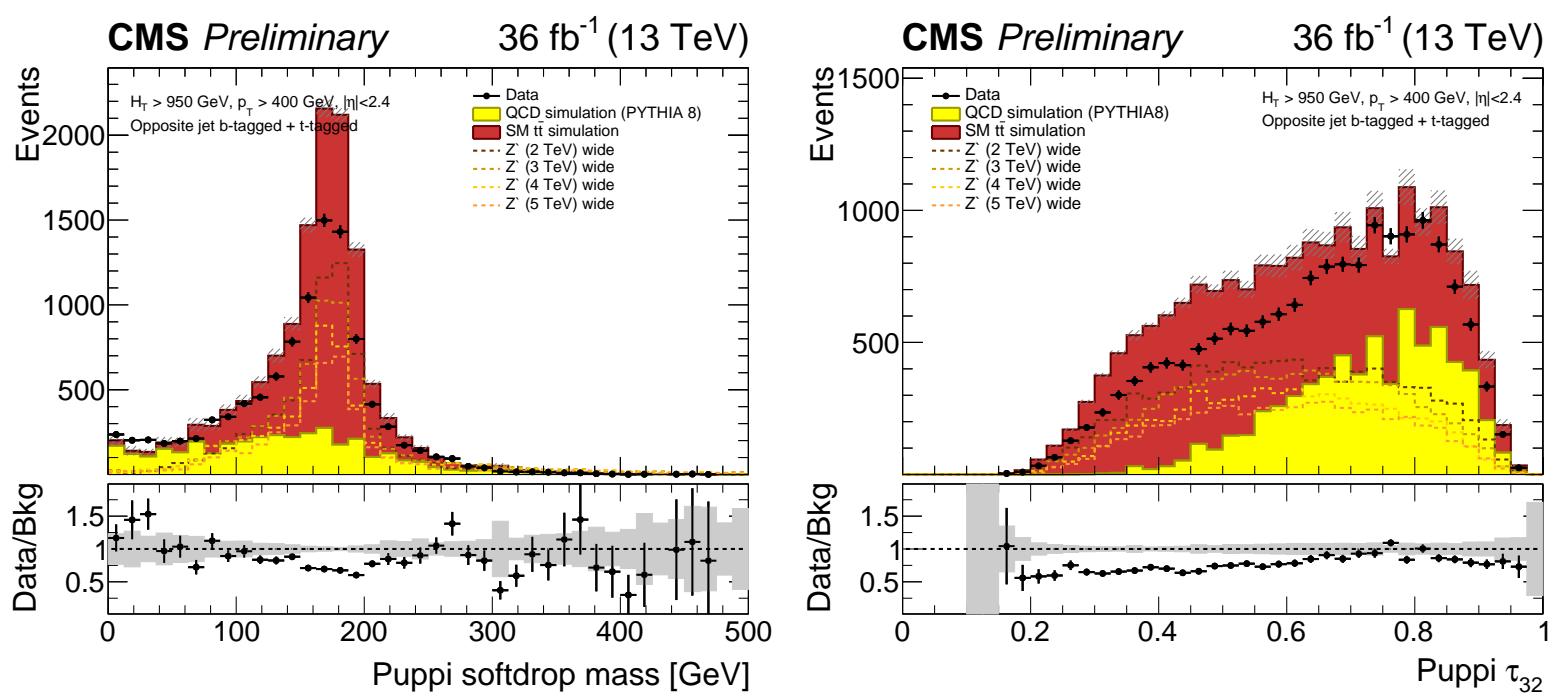

Figure 6.2.7: Comparison of data, background simulation, and signal simulation, for the quantities used in CMSTT V2H: AK8 PUPPI jet softdrop mass (left) and AK8 PUPPI jet $\tau_{3} / \tau_{2}$ value (right). Each plot is made requiring the CMSTT V2H selection with the exception of the variable being plotted.

scale factors are applied. Scale factors provided by the BTV POG (CMS b-tagging group) are used.

In order to properly account for the migration of events from one b-tag category to another when applying the scale factors, a "subjet-by-subjet updating method" is employed. This method is defined as follows: each subjets's b-tagging status is upgraded or downgraded based on the scale factor and a random number. For scale factors less than 1, b-tagged subjets are downgraded based on the fraction $f=1-\mathrm{SF}$, while for scale factors greater than 1, untagged subjets are upgraded based on the fraction $f=(1-\mathrm{SF}) /\left(1-1 / \epsilon_{M C}\right)$, where $\epsilon_{M C}$ is the b-tagging Monte Carlo efficiency. With this procedure the fraction of events in each b-tagging category is modified. The b-tag efficiency for different subjet hadron flavors $\left(\epsilon_{M C}\right)$ has been measured in QCD Monte Carlo (Fig. 6.2.8). Subjet b-tag scale factors depend on the flavor of the subjet, and therefore the hadron flavor of each subjet is used when finding the correct scale factor.

Due to limited statistics when measuring the b-tag SF, the BTV POG recommends that 
the scale factor uncertainty should be doubled for subjets with $p_{T}$ outside of the range $20-$ $1000 \mathrm{GeV}$ for udsg flavor subjets and outside of the range $30-450 \mathrm{GeV}$ for b and c flavor subjets.

An AK8 jet is considered to contain a subjet b-tag if, after applying the subjet-by-subjet updating procedure, the jet contains at least one b-tagged subjet. The events are then categorized based on the number of jets containing at least one subjet b-tag (see Section $6.2 .8)$.

\subsubsection{Signal Region and Event Categorization}

Given the back-to-back high $p_{T}$ dijet events selected by the preselection (Section 6.2.5), our signal region is defined to be events containing two top tagged jets.

It is observed that for high dijet mass, the rapidity difference $(\Delta y)$ between the two top tagged jets can be used to further discriminate signal from background (Figure 6.2.9). The signal region events are therefore categorized into the regions defined by $\Delta y<1$ and $\Delta y>1$.

Subjet b-tagging can also be used to discriminate signal from background. Therefore, events are further categorized based on the number of jets containing a subjet b-tag $(0,1$, or 2).

In order to optimize the selection, the two subjet b-tagging working points and three top tagging working points provided by the CMS Physics Object Groups are considered.

The b-tag working points are:

- Medium operating point (M): CSVv2 > 0.8484

- Loose operating point (L): CSVv2 >0.5426

The top tagging working points are:

- Working point A : $105 \mathrm{GeV}<m_{S D}<210 \mathrm{GeV}$ and $\tau_{3} / \tau_{2}<0.80$ (loose, $\epsilon_{B}=3 \%$ )

- Working point B : $105 \mathrm{GeV}<m_{S D}<210 \mathrm{GeV}$ and $\tau_{3} / \tau_{2}<0.65\left(\right.$ medium $\left.\epsilon_{B}=1 \%\right)$ ) 

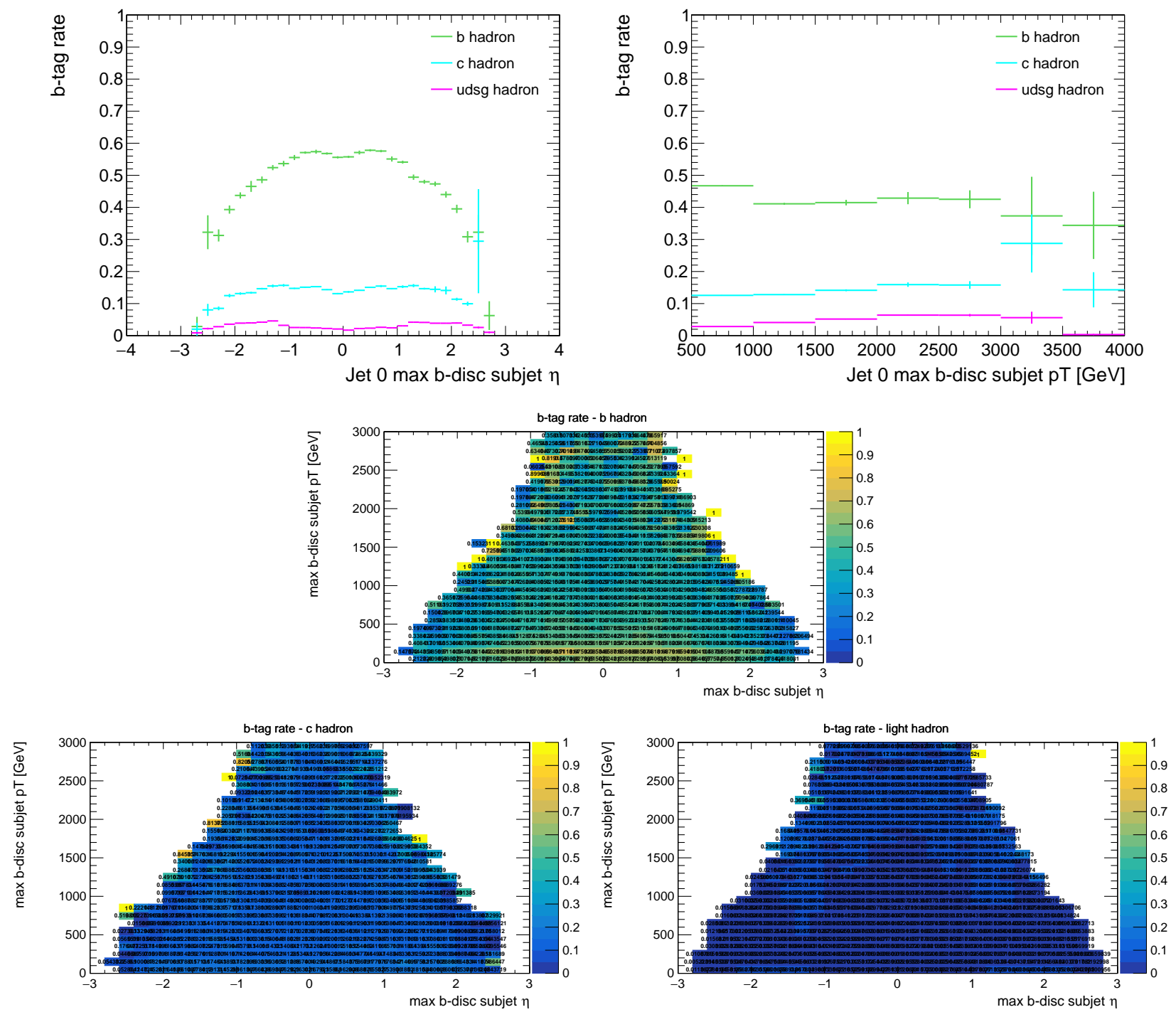

Figure 6.2.8: b-tag rate of the subjet with the maximum b-discriminant for the medium operating point as measured in a simulated QCD sample. (a) The b-tag rate as a function of the subjet $\eta$ for subjets of different hadron flavor. (b) The b-tag rate as a function of the subjet $p_{T}$ for subjets of different hadron flavor. (c) The b-tag rate for a given subjet $p_{T}$ and $\eta$ for subjets originating from b-hadrons. (d) The b-tag rate for a given subjet $p_{T}$ and $\eta$ for subjets originating from c-hadrons. (e) The b-tag rate for a given subjet $p_{T}$ and $\eta$ for subjets originating from udsg-hadrons. 

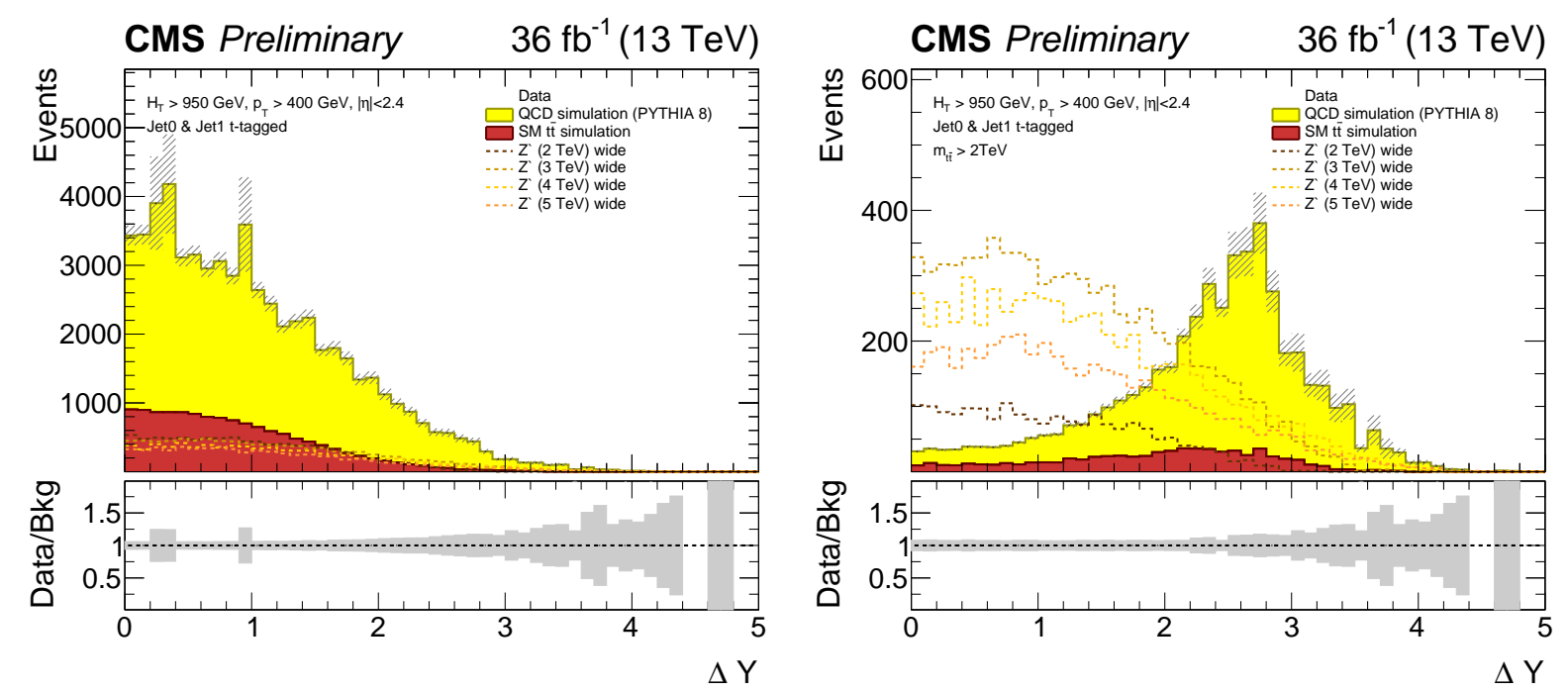

Figure 6.2.9: Dijet rapidity difference after requiring two top tagged jets: (a) $\Delta y$ (inclusive in $\left.m_{t t}\right)$, (b) $\Delta \mathrm{y}\left(m_{t t}>2 \mathrm{TeV}\right)$.

- Working point $\mathrm{C}: 105 \mathrm{GeV}<m_{S D}<210 \mathrm{GeV}$ and $\tau_{3} / \tau_{2}<0.54\left(\right.$ tight $\left.\epsilon_{B}=0.3 \%\right)$ )

Given the six event categories, discovery significance (Figure 6.2.10) and expected limits (Figure 6.2.11) are calculated for each combination of b-tag and top tag working points. The medium b-tag working point and top tagging working point $\mathrm{B}$ are found to be the best choice when considering both metrics. Therefore, this combination of working points is chosen.

These categories uses in this analysis are summarized in Table 6.5.

\begin{tabular}{lllll}
$\begin{array}{l}\text { Event Cat- } \\
\text { egory }\end{array}$ & $\begin{array}{l}\text { PUPPI Softdrop } \\
\text { jet mass window }\end{array}$ & $\tau_{3} / \tau_{2}$ Cut & $|\Delta y|$ Cut & $\begin{array}{l}\text { Number of jets } \\
\text { with a CSVM b- } \\
\text { tagged subjet }\end{array}$ \\
\hline A & $105-210$ & $<0.65$ & $<1.0$ & 0 \\
B & $105-210$ & $<0.65$ & $<1.0$ & 1 \\
C & $105-210$ & $<0.65$ & $<1.0$ & $\geq 2$ \\
D & $105-210$ & $<0.65$ & $>1.0$ & 0 \\
E & $105-210$ & $<0.65$ & $>1.0$ & 1 \\
F & $105-210$ & $<0.65$ & $>1.0$ & $\geq 2$
\end{tabular}

Table 6.5: Event selections defining the six categories used in the analysis. 

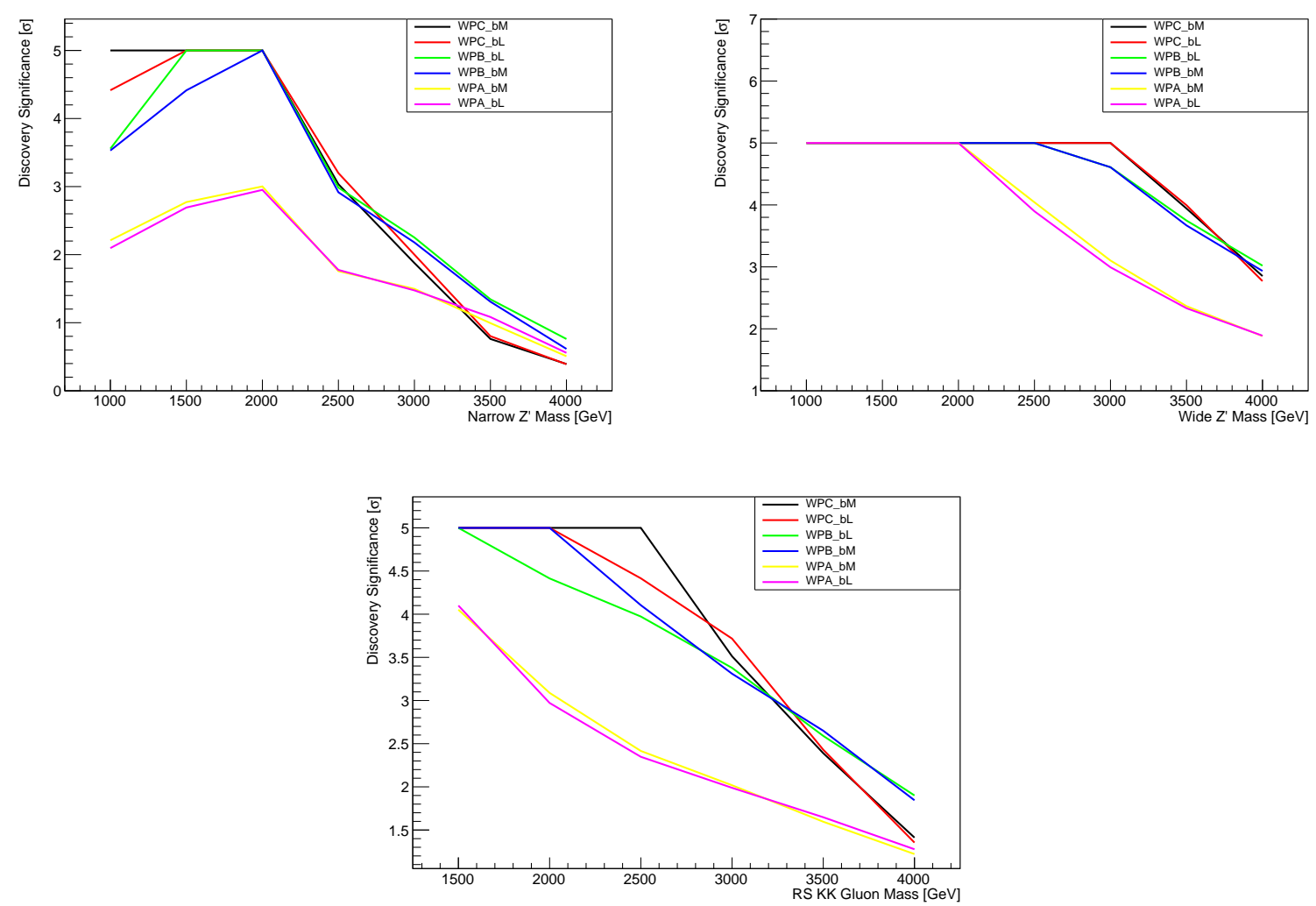

Figure 6.2.10: Discovery significance for the full analysis using the 6 event categories for the different top tagging working points A, B, C and b-tag working points bL (Loose) or bM (Medium). The three models considered are a $Z^{\prime}$ boson whose width is $1 \%$ of its mass (upper left), a 10\% width $Z^{\prime}$ boson (upper right), and an RS KK gluon (bottom). Working point $\mathrm{B}$ is optimal at high masses, which have not yet been excluded by previous searches. 

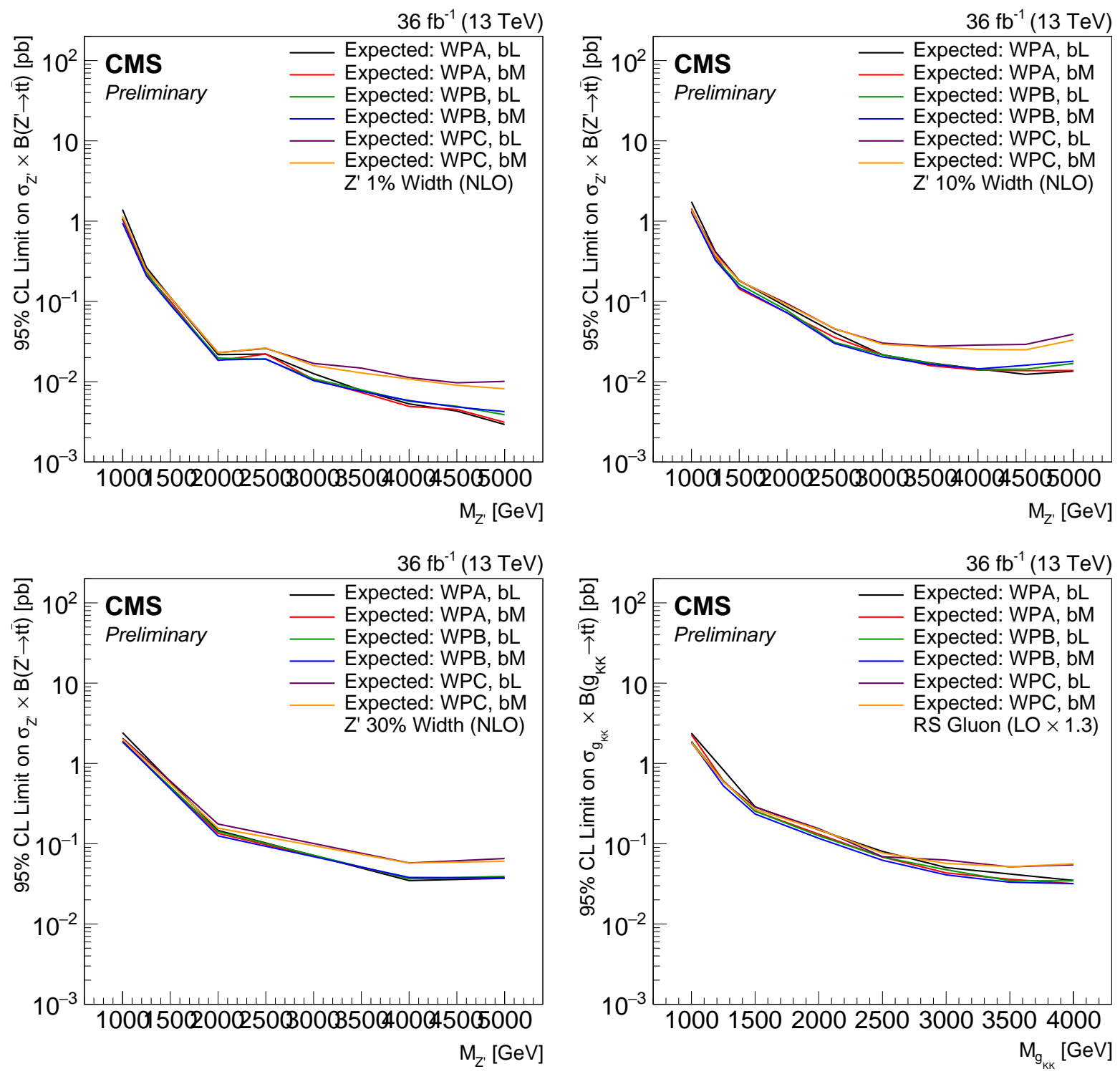

Figure 6.2.11: Expected limits for the full analysis using the 6 event categories for the different top tagging working points $\mathrm{A}, \mathrm{B}, \mathrm{C}$ and b-tag working points bL (Loose) or bM (Medium). The four models considered are a $Z^{\prime}$ boson whose width is $1 \%$ of its mass (upper left), a $10 \%$ width $Z^{\prime}$ boson (upper right), a $30 \%$ width $Z^{\prime}$ boson (lower left), and an RS KK gluon (lower right). 


\section{Chapter 7}

\section{Background Estimation and Event Kinematics}

This analysis has two sources of background: multijet events originating from nontop QCD interactions (nontop multijet, i.e., NTMJ), that will be determined from data, and SM $t \bar{t}$ events, determined from simulated events. This chapter describes the methods for estimating the various background contributions.

\subsection{Data-derived Backgrounds}

For the nontop multijet estimate, a data-derived technique is used. This technique is similar to that described in Ref. [63]. The method involves selecting a sample with a low SM $t \bar{t}$ contribution. This is done by inverting the top tagging requirements on one selected jet (antitag) and determining the top tagging rate for the second jet (probe). This 'antitag-and-probe' method yields a per-jet mistag rate that is then applied to each jet in the event to determine the estimated number of NTMJ background events. This mistag rate is parameterized as a function of jet $p$ (not $p_{T}$, but the full momentum) and is measured separately for events falling into each of the 3 b-tag categories inclusive in delta rapidity.

The mistag rate is determined by the following procedure: 
1. Dijet events are selected, in which the leading two AK8 jets are required to have $p_{T}$ greater than $400 \mathrm{GeV}$. The two jets also have additional kinematic requirements similar to those used in the main selection: hemispheric separation $(|\Delta \phi|>2.1)$ is required, as well as requirements that the event fall into one of the b-tag and rapidity difference bins as described in table 6.5.

2. One jet is selected at random - this jet is "antitagged" to select an enriched sample of NTMJ events. This jet must pass an inverted n-subjettiness selection $\left(\tau_{3} / \tau_{2}>0.65\right)$ and the softdrop jet mass must be in the top mass window $(105-210 \mathrm{GeV})$.

3. Once the antitagged jet is selected, the second jet is then used as the probe jet.

4. The top tagging rate of the probe jet is taken as the mistag rate for the algorithm. The mistag rate is measured as a function of jet $p$ and is measured separately for events falling into each of the three b-tag categories. Due to the inversion of the $\tau_{3} / \tau_{2}$ cut, the data sample is dominated by multijet events in the right kinematic regime, and is therefore an appropriate control region to extract the mistag rate.

The mistag rate is measured both in an NTMJ rich data sample and in $t \bar{t} \mathrm{MC}$. The $t \bar{t}$ contamination from the tag-and-probe distributions is subtracted before calculating the mistag rate. Table 7.1 shows the the antitag-and-probe event yields for $t \bar{t} \mathrm{MC}$ and data in each of the six event categories. Figure 7.1.1 shows the mistag rate as measured in data for each b-tag category and Figure 7.1.2 compares the mistag rate measured in data to QCD simulation. Better data/MC agreement is seen for the QCD samples simulated with PYTHIA8. This is not unexpected, as problems have been observed in some of the other samples simulated with MADGRAPH.

Once the mistag rate is determined from the NTMJ control sample, it is used to estimate the normalization and shape of NTMJ events passing the final event selection. To do this, a "pre-tagged" region, which contains events with at least one top-tagged jet, is used. In order to avoid bias, one of the two leading jets, which pass the preselection, is randomly selected 


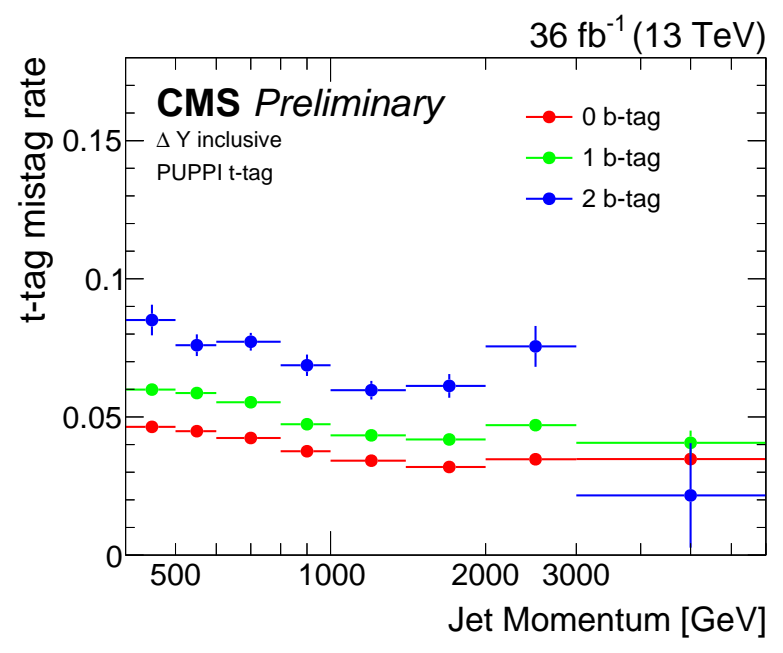

Figure 7.1.1: Top tagging mistag rate as measured with an antitag-and-probe procedure separately for each b-tag category.

and is asked to pass the CMSTT V2H selection described above. If the randomly chosen jet is indeed top-tagged, this event is used and weighted by the appropriate mistag rate after finding the appropriate bin of the second jet $p$ value. There is no selection applied on this second jet, and if the randomly chosen first jet is not tagged, the event is not used to model the multijet background.

To model the individual signal region categories with differing numbers of b-tagged jets and rapidity separations, the same selections are applied to the events weighted by the mistag rate as those applied to the signal region. For example, in the 1 b-tag category with $|\Delta y|<1.0$, this weighted event is required to have exactly 1 b-tagged jet (it can be either the already tagged jet or the second jet used to determine the mistag rate), and to also have the two jets separated by a rapidity difference of no more than 1.0 units. Analogous selections are applied for the remaining signal regions.

Using this single-tagged control region without any requirements on the second jet leads to an overlap between the signal region and the region used to estimate the multijet background. To remove the effects of double counting, the $t \bar{t}$ contribution is subtracted from the multijet estimate. This is done by evaluating the mistag weighting procedure described above on 

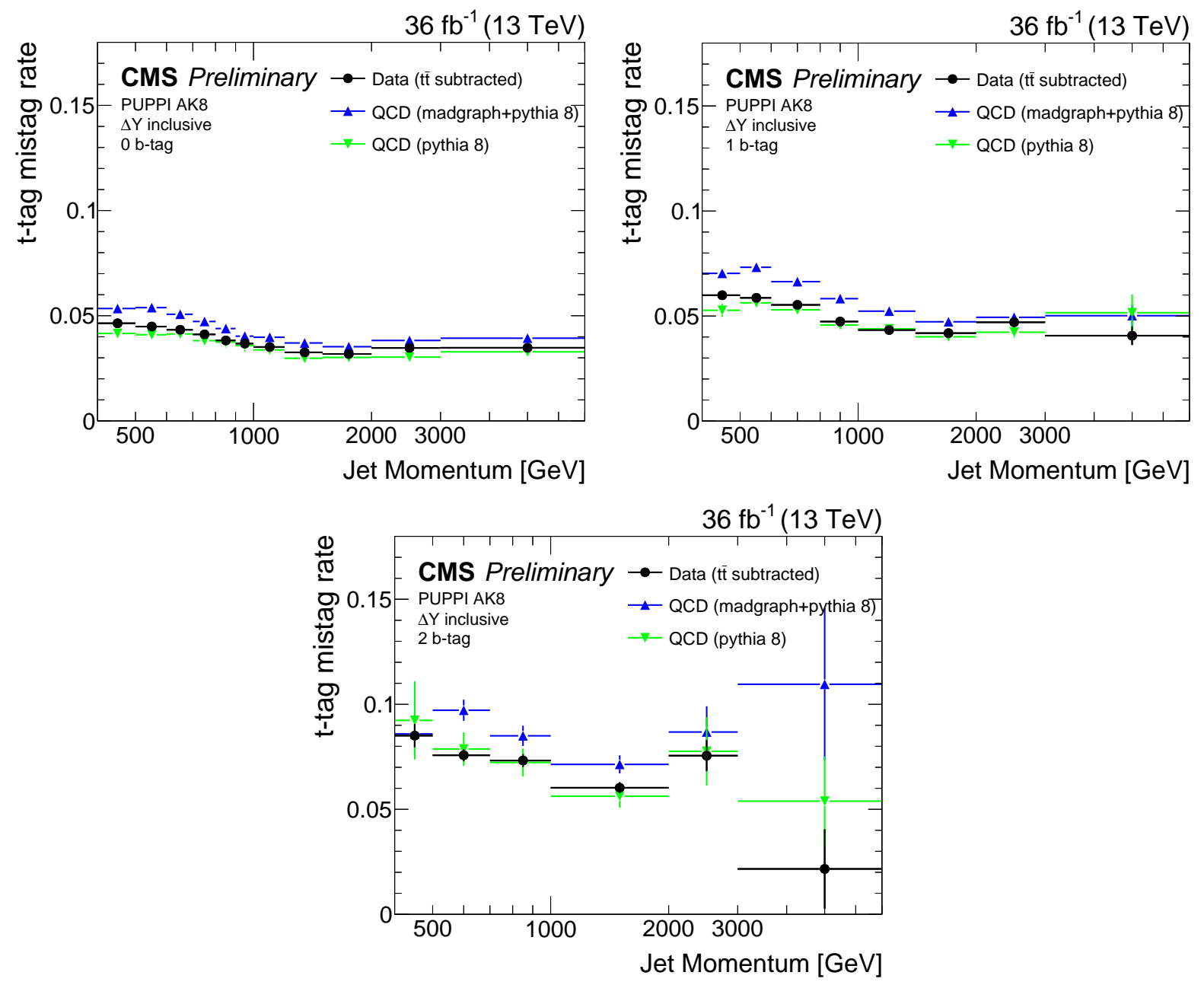

Figure 7.1.2: Top tagging mistag rate measured in data and in QCD simulation with the antitag-and-probe procedure: (a) 0 b-tag category, (b) 1 b-tag category, (c) 2 b-tag category. 


\begin{tabular}{llrr} 
Sample & Event Category & Probe event yields & Tag event yields \\
\hline$t \bar{t}$ & $\Delta \mathrm{Y}>1,0 \mathrm{~b}$-tag & 3104 & 594 \\
$t \bar{t}$ & $\Delta \mathrm{Y}>1,1 \mathrm{~b}$-tag & 4670 & 1200 \\
$t \bar{t}$ & $\Delta \mathrm{Y}>1,2 \mathrm{~b}$-tag & 1202 & 513 \\
$t \bar{t}$ & $\Delta \mathrm{Y}<1,0 \mathrm{~b}$-tag & 3976 & 864 \\
$t \bar{t}$ & $\Delta \mathrm{Y}<1,1 \mathrm{~b}$-tag & 6614 & 1848 \\
$t \bar{t}$ & $\Delta \mathrm{Y}<1,2 \mathrm{~b}$-tag & 2051 & 905 \\
Data & $\Delta \mathrm{Y}>1,0 \mathrm{~b}$-tag & 1710380 & 67049 \\
Data & $\Delta \mathrm{Y}>1,1 \mathrm{~b}$-tag & 370824 & 19570 \\
Data & $\Delta \mathrm{Y}>1,2 \mathrm{~b}$-tag & 21486 & 1912 \\
Data & $\Delta \mathrm{Y}<1,0 \mathrm{~b}$-tag & 1685670 & 68945 \\
Data & $\Delta \mathrm{Y}<1,1 \mathrm{~b}$-tag & 375487 & 21168 \\
Data & $\Delta \mathrm{Y}<1,2 \mathrm{~b}$-tag & 23202 & 2477
\end{tabular}

Table 7.1: Data and $t \bar{t}$ yields for the tag-and-probe histograms.

the simulated $t \bar{t}$ events, to find the amount of 'mistagged $t \bar{t}$ '. Generally, this 'mistagged $t \bar{t}$ ' makes up about 1-2\% of the NTMJ background estimate in the 0 b-tag event regions, and about $6-10 \%$ in the other regions.

As a final step to the shape determination of the NTMJ estimate, a so-called 'massmodified' procedure is used to account for the fact that the second jet, having no tag selection applied, will have different kinematics than the jets in the signal region, which have a jet mass requirement. To mimic the kinematics of the signal region, the mass of this second jet is set 'by hand' to follow a distribution of jet masses from simulated QCD events in the same window used for the signal region selection, $105<m_{S D}<210 \mathrm{GeV}$. The specific mass is chosen by drawing randomly from this distribution of QCD jet masses. Figure 7.1.3 shows the softdrop mass distributions used in the mass-modified procedure for the two leading jets.

\subsection{Closure Tests for the CMS Top Tagger V2H Anal- ysis}

To validate the method described above to estimate the NTMJ background, the background estimate procedure is performed using a sideband tag definition in data. Here, the same 


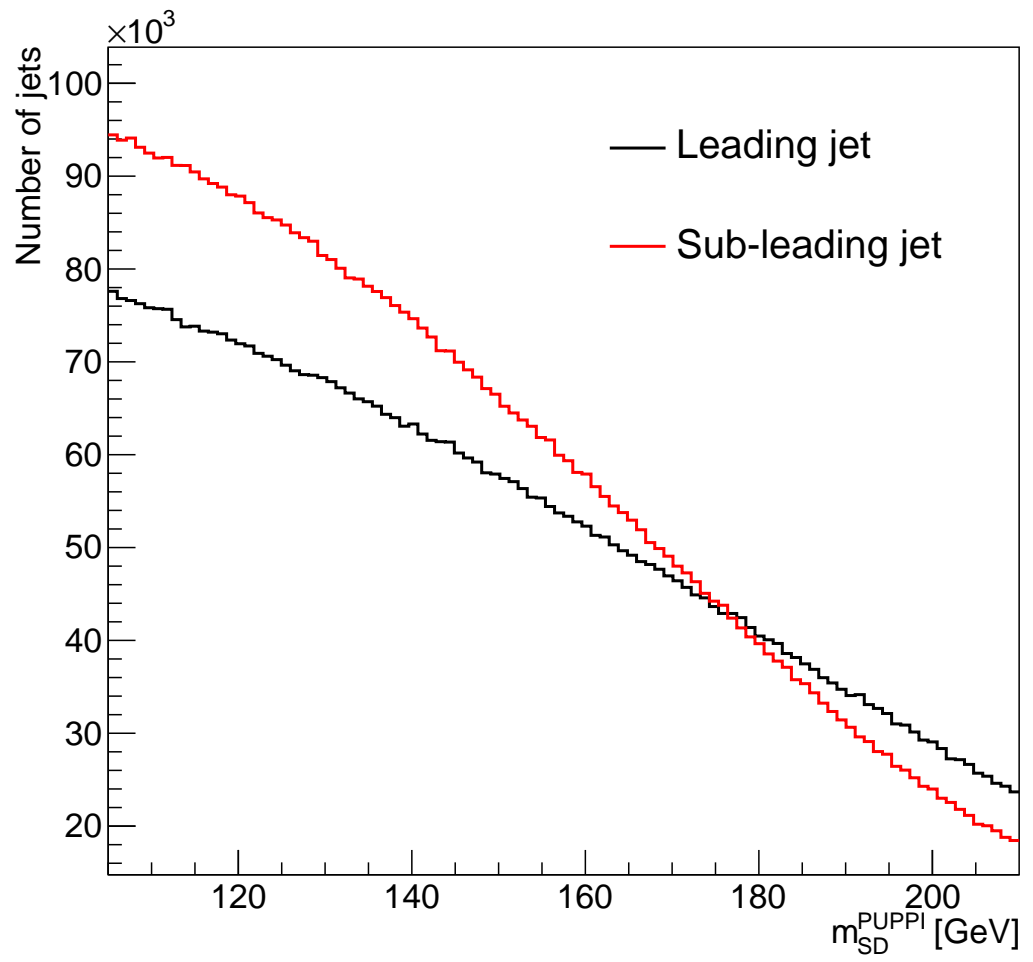

Figure 7.1.3: Soft drop mass distributions for the event leading and sub-leading jet as measured in the data preselection. These distributions are used for the mass-modified procedure. 
event categories and n-subjettiness selection, but a separate softdrop mass selection (40-105 $\mathrm{GeV}$ ), are used. The mistag rate for this tag definition is shown in Figure (7.2.1). Using this tag definition, good closure is observed between the background estimate and the double sideband tagged signal region in the individual event categories (Figure 7.2.2).

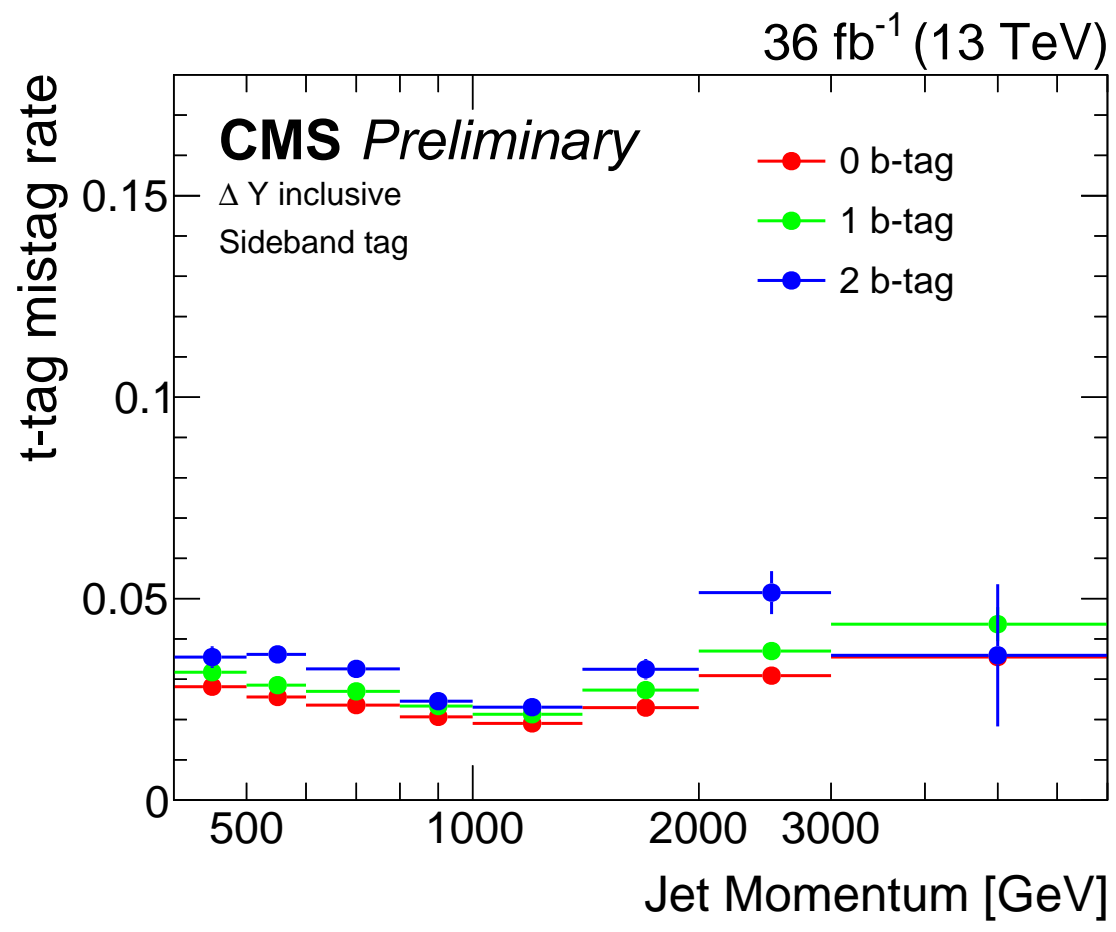

Figure 7.2.1: Sideband tag mistag rate as measured with an antitag-and-probe procedure separately for each b-tag category

\subsection{Event Kinematics}

In this section, some event kinematics are examined after the selection has been performed. Figure 7.3.1 shows comparisons of data and the data-driven background estimate. Comparisons of data to QCD Monte Carlo can be found in Appendix B. In the analysis results, the NTMJ background is estimated using a data-driven technique, as described in a previous section. The $t \bar{t}$ Monte Carlo is scaled based on the top- and b-tagging scale factors when appropriate. An additional scale factor is applied to take into account the measured differ- 

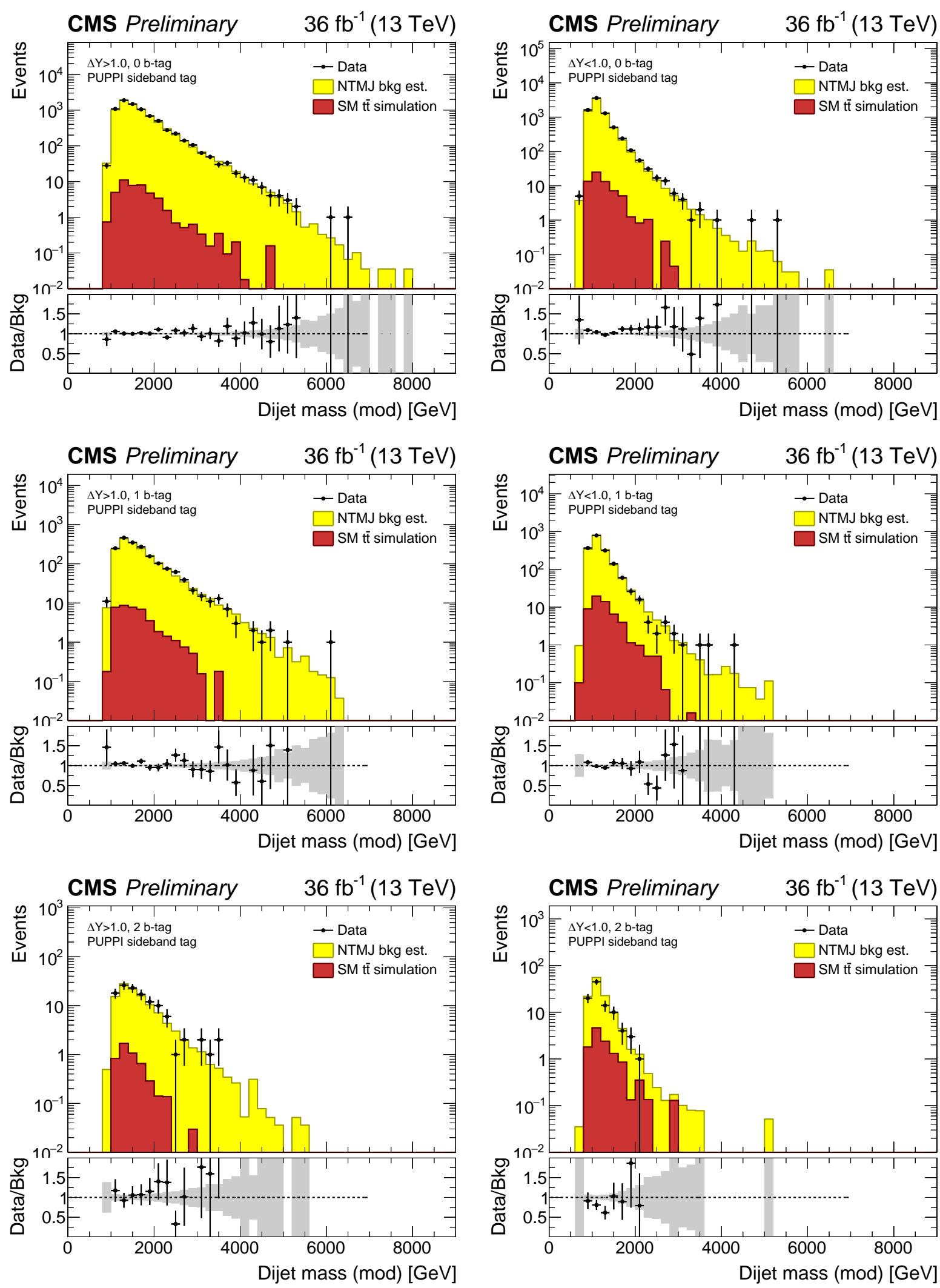

Figure 7.2.2: Closure test for the $0+1+2$ b-tag categories in the two delta rapidity regions using the data sideband tag definition. 
ence between the high- $p_{T} t \bar{t}$ cross section in data and simulation. This scale factor (0.9) is taken from the normalized parton-level differential cross section measurement made by CMS (TOP-14-002) in the $600 \mathrm{GeV} p_{T}$ bin. This is consistent with NNLO corrections to the NLO differential cross section in this region [73].
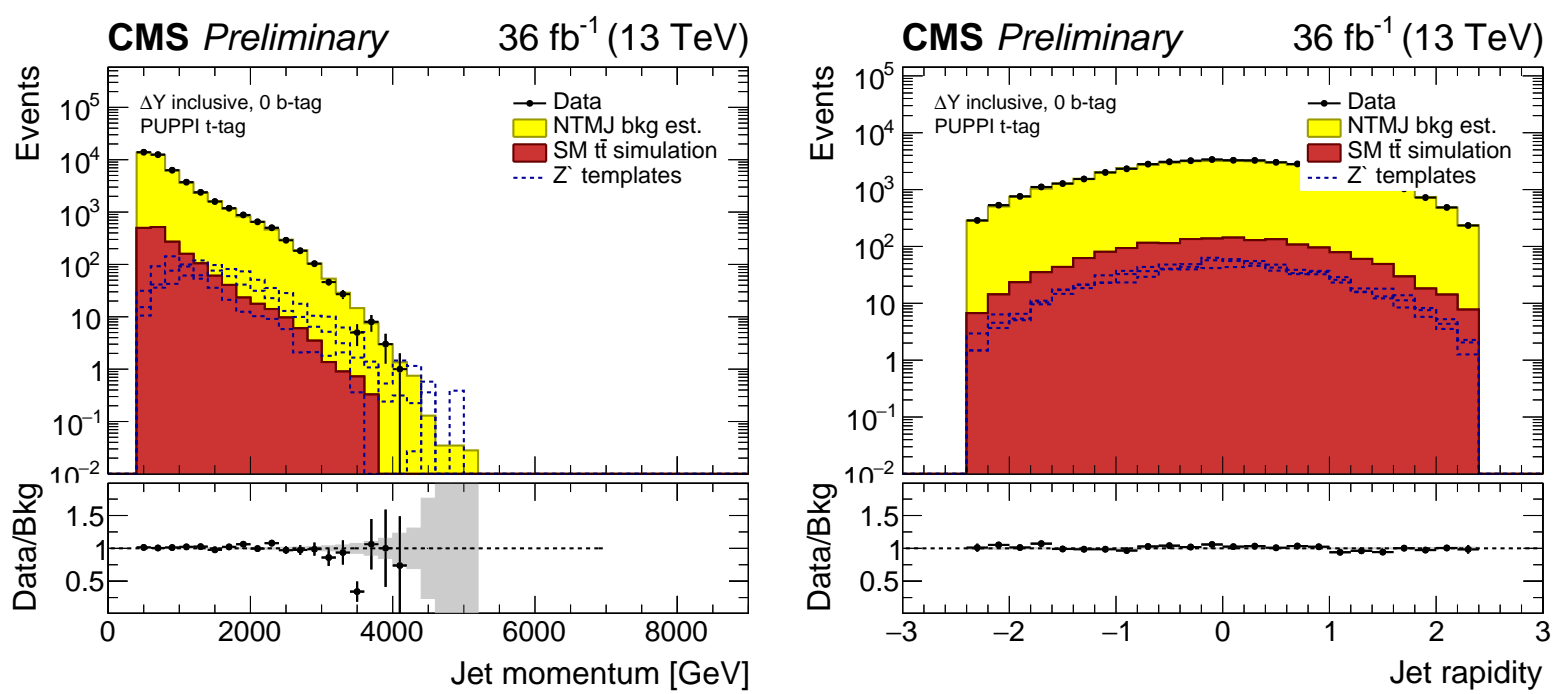

Figure 7.3.1: Kinematic comparisons showing $t \bar{t} \mathrm{MC}$ and data-driven NTMJ compared to data for the 0 b-tag category: (left) jet momentum, (right) jet rapidity.

Table 7.2 shows the expected numbers of events for each of the background processes for each of the six signal categories used in the analysis. The NTMJ background entry represents the data-driven estimate. 


\begin{tabular}{lrrr} 
& \multicolumn{3}{c}{$|\Delta y|<1.0$} \\
Process & 0 b-tags & $\mathbf{1}$ b-tag & 2 b-tags \\
\hline NTMJ & $21174 \pm 298$ & $6369 \pm 82$ & $398 \pm 9$ \\
SM $t \bar{t}$ & $1561 \pm 755$ & $4100 \pm 2035$ & $2600 \pm 1339$ \\
\hline Total Background & $22735 \pm 812$ & $10469 \pm 2037$ & $2998 \pm 1339$
\end{tabular}

\begin{tabular}{lrrr} 
& \multicolumn{3}{c}{$|\Delta y|>1.0$} \\
Process & 0 b-tags & 1 b-tag & 2 b-tags \\
\hline NTMJ & $20551 \pm 101$ & $6153 \pm 33$ & $435 \pm 7$ \\
SM $t \bar{t}$ & $1007 \pm 386$ & $2428 \pm 940$ & $1442 \pm 573$ \\
\hline Total Background & $21558 \pm 399$ & $8581 \pm 940$ & $1877 \pm 573$
\end{tabular}

Table 7.2: Expected background yields for the six event categorizations used in the final analysis selection. Errors include both the statistical and systematic components. 


\section{Chapter 8}

\section{Systematic Uncertainties}

Several sources of systematic uncertainty affect both the normalization of event yields in the analysis and the shapes of the $t \bar{t}$ invariant mass distribution for various processes. These systematic uncertainties are evaluated on both the simulated samples and the data-driven method used to estimate the NTMJ background contribution. This section details the individual contributions to the total systematic uncertainty of the analysis. Table 8.1 shows a list of the systematic uncertainties.

\begin{tabular}{lcc} 
Systematic Uncertainty & Value & Type \\
\hline b-Tag SF Uncertainty & $\pm 1 \sigma$ & Rate + Shape \\
CMS Top Tagger SF Uncertainty & $+25 \%-9 \%$ & Rate \\
Jet Energy Corrections & $\pm 1 \sigma$ & Rate + Shape \\
Jet Energy Resolution & $\pm 1 \sigma$ & Rate + Shape \\
Luminosity & $2.5 \%$ & Rate \\
PDF Uncertainty & $\pm 1 \sigma$ & Rate + Shape \\
Pileup Uncertainty & $\pm 1 \sigma$ & Rate + Shape \\
Top $p_{T}$ Reweighting Uncertainty & $\pm 1 \sigma$ & Rate + Shape \\
$t \bar{t} Q^{2}$ Uncertainty & $\pm 1 \sigma$ & Rate + Shape \\
$t \bar{t}$ Cross Section & $8 \%$ & Rate \\
Mistag Rate Uncertainty & $\pm 1 \sigma$ & Rate + Shape \\
QCD Modified Mass Procedure & $\pm 1 \sigma$ & Rate + Shape
\end{tabular}

Table 8.1: Summary of systematic uncertainties applied to the analysis. 


\subsection{Top Tagging and Subjet b-Tagging Scale Factor Uncertainties}

All jets in the signal selection are top-tagged and therefore the top tagging scale factor is applied as an overall normalization scale factor to the final selection (the scale factor is applied twice, once per jet). The associated systematic uncertainty, based on the scale factor uncertainty, is found to be $+25 \%-9 \%$ for each event.

The subjet b-tagging scale factor is applied on a jet-by-jet basis (see Section 6.2.7). The subjet b-tagging systematic uncertainty is obtained by applying a $\pm 1 \sigma$ variation of the $\mathrm{SF}$ value.

\subsection{Jet Energy Scale}

The effect of the jet energy scale uncertainty on the simulated samples is evaluated. This is done by varying the jet four-momentum up and down by the jet energy scale uncertainty [74, which is approximately 5\%. Both $p_{T^{-}}$and $\eta$-dependent corrections to the jet energies are included, combined with uncertainties due to the difference in the $W$ mass for data and Monte Carlo, along with uncertainties on the response of the pruning algorithm for jets in data and simulation. This combination of uncertainties is described in [75]. This results in a shape difference in the $m_{t \bar{t}}$ spectrum which depends on the invariant mass itself. For low masses, the effect is large since the jets are close to the $p_{T}$ cut of $400 \mathrm{GeV}$. Varying the four-momenta causes jets to fall below the cut, or be promoted above the cut - increasing

or decreasing acceptance. For higher invariant masses, the jets generally have higher $p_{T}$ and this effect is smaller. This systematic uncertainty is applied to both the signal samples and the SM $t \bar{t}$ sample. 


\subsection{Jet Energy Resolution}

Differing jet energy resolutions between simulated events and data events are accounted for by randomly "smearing" the simulated jet energies. The recommended eta-dependent smearing to jets, as listed in Table 8.2 , is applied and the errors are used to form systematic shape uncertainty templates. It is assumed that the jet energy resolution for subjets is the same as that for the hard jets. Therefore, the variation of the subjet $p_{T}$ is included in the shape uncertainties. This systematic uncertainty is applied to both the signal samples and the SM $t \bar{t}$ sample.

\begin{tabular}{cc}
$|\eta|$ Range & Smearing Factor \\
\hline $0.0<\eta<0.5$ & $1.109 \pm 0.008$ \\
$0.5<\eta<0.8$ & $1.138 \pm 0.013$ \\
$0.8<\eta<1.1$ & $1.114 \pm 0.013$ \\
$1.1<\eta<1.3$ & $1.123 \pm 0.024$ \\
$1.3<\eta<1.7$ & $1.084 \pm 0.011$ \\
$1.7<\eta<1.9$ & $1.082 \pm 0.035$ \\
$1.9<\eta<2.1$ & $1.140 \pm 0.047$ \\
$2.1<\eta<2.3$ & $1.067 \pm 0.053$ \\
$2.3<\eta<2.5$ & $1.177 \pm 0.041$ \\
$2.5<\eta<2.8$ & $1.364 \pm 0.039$ \\
$2.8<\eta<3.0$ & $1.857 \pm 0.071$ \\
$3.0<\eta<3.2$ & $1.328 \pm 0.022$ \\
$3.2<\eta<5.0$ & $1.16 \pm 0.029$
\end{tabular}

Table 8.2: Systematic smearing factors applied to individual jets to obtain shape templates used for the jet energy resolution systematic.

\subsection{Parton Distribution Function Uncertainties}

The effect of the parton distribution function (PDF) uncertainty on the simulated samples is evaluated. The RMS of the NNPDF3.0 PDF weights are found and used to weight events up and down. This results in a shape difference in the $m_{t \bar{t}}$ spectrum. This systematic uncertainty is applied to both the signal samples and the SM $t \bar{t}$ sample. 


\subsection{Pileup Uncertainties}

The effect of the pileup uncertainty on the simulated samples is evaluated by reweighting the Monte Carlo simulation with the data pileup distribution, based on a minimum bias cross section of $69 \mathrm{mb}$. The uncertainty on this cross section, $5 \%$, is used to vary the pileup distribution up and down. This results in a shape difference in the $m_{t \bar{t}}$ spectrum. This systematic uncertainty is applied to both the signal samples and the SM $t \bar{t}$ sample.

\subsection{Top $p_{T}$ Reweighting Uncertainties}

The systematic uncertainty due to the top $p_{T}$ reweighting of the simulated samples is computed by taking the difference between the $m_{t \bar{t}}$ distributions with and without top $p_{T}$ reweighting applied. This results in a shape difference in the $m_{t \bar{t}}$ spectrum.

\subsection{SM $t \bar{t}$ Uncertainties}

The uncertainties applied exclusively to SM $t \bar{t}$ events include two contributions: a rate-only component due to the uncertainty in the $t \bar{t}$ cross section, which is taken to be $8 \%$ [76], and a second shape component for uncertainties in the factorization and normalization scale $\left(Q^{2}\right)$ uncertainties. The $Q^{2}$ uncertainties are evaluated by finding the envelope of the $Q^{2}$ weights (variations of $\mu_{R}$ and $\mu_{F}$ by factors of 2 or 0.5 ), which is then used to weight events up and down. This results in a shape difference in the $m_{t \bar{t}}$ spectrum.

\subsection{Multijet Background Uncertainties}

The mistag rate uncertainty, shown in Figure 7.1.1, contains statistical uncertainties which are propagated to the NTMJ background estimation. The systematic uncertainty associated with the 'mass-modified' procedure, which is used to correct kinematic bias in the background 
estimation, is computed by taking half the difference between the uncorrected background estimate and and the 'mass-modified' background estimate. 


\section{Chapter 9}

\section{Statistical Interpretation and Results}

In this section, expected limits for the blinded 2016 analysis are presented, along with the results of the 2015 analysis, for comparison. A template-based shape analysis is performed using the distributions of the candidate top pair invariant mass. The background-only distributions from the 2016 analysis are shown in Figs. 9.0.1 and 9.0.2. The distributions input to the limit setting program have a variable binning, which is chosen to best accommodate the background uncertainties.

The Theta software package [77] is used to produce Bayesian 95\% confidence level (CL) upper limits on the cross sections for the four signal models, as a function of the new heavy particle mass. This method assumes a flat prior pdf in the signal cross section. A Poisson model is used for each bin in the $m_{t \bar{t}}$ distribution, with the final likelihood composed of the product of these Poisson probabilities for each bin in the $m_{t \bar{t}}$ distribution. For a single bin, the mean of this distribution is shown in Equation 9.0.1.

$$
\mu_{i}=\sum_{k} \beta_{k} \cdot T_{k, i}
$$

The index $k$ represents each physics process contributing to the bin $i, T$ represents the number of expected events for the process $k$ in bin $i$, and the factor $\beta$ is the Poisson mean for the individual process $k$. With this per-bin formula, the full likelihood can be formed as 

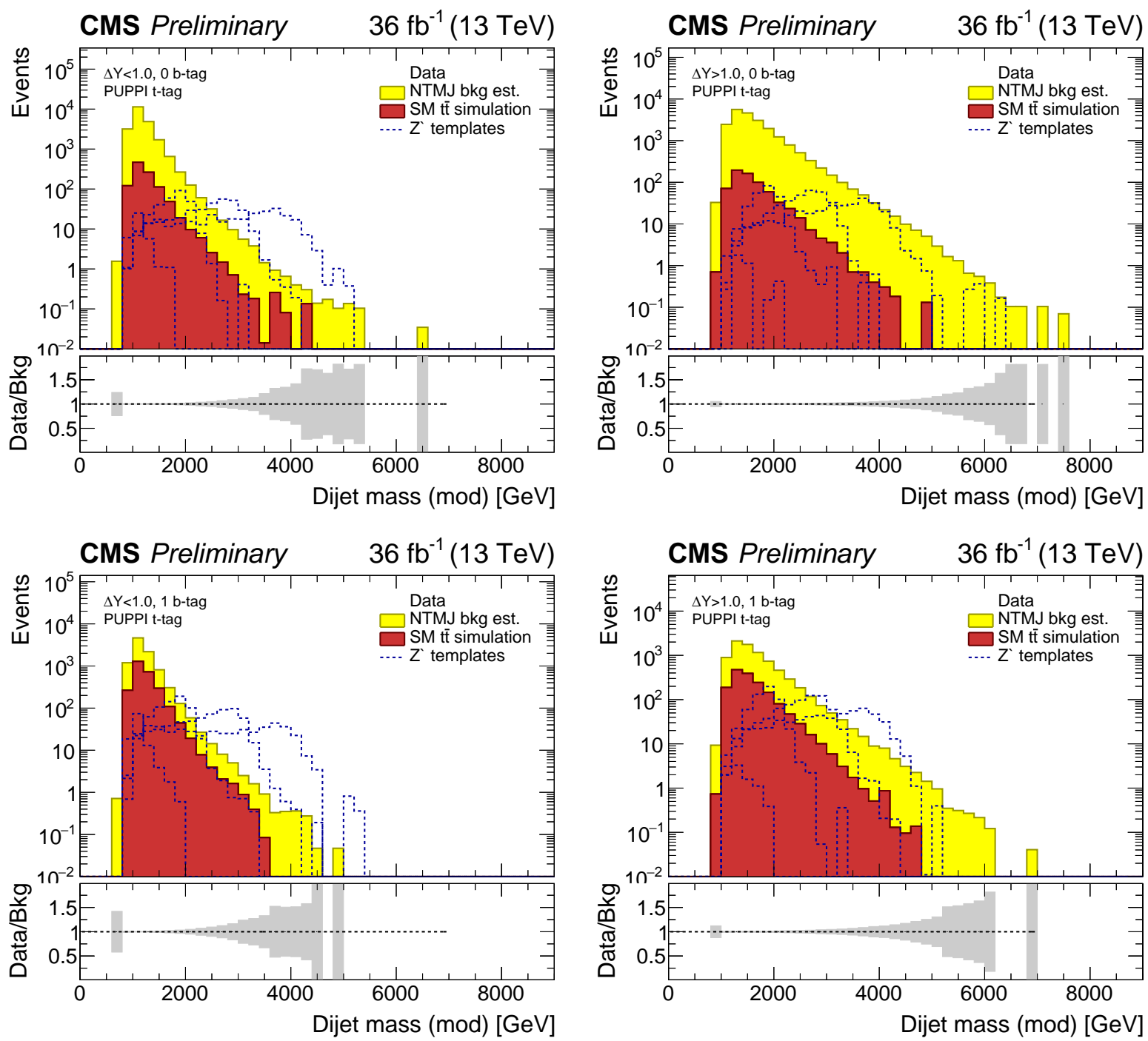

Figure 9.0.1: Background-only distributions of $m_{t \bar{t}}$ for four of the six signal regions of the 2016 analysis, with the $\Delta y<1.0$ categories shown in the left column and the $\Delta y>1.0$ categories in the right. The number of b-tags in the plots increase from zero in the first row to one b-tag in the second row. The shaded region corresponds to the combined systematic and statistical uncertainties on the background model. 

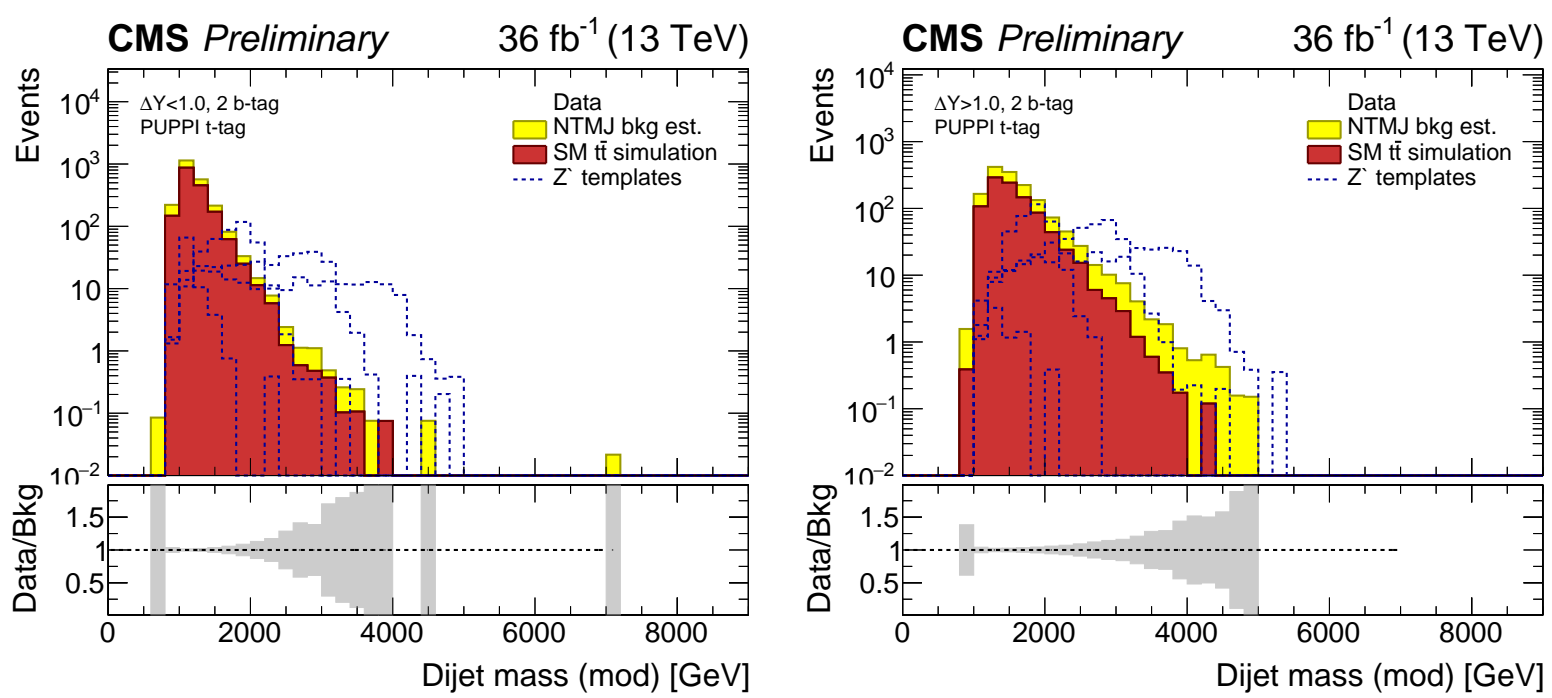

Figure 9.0.2: Background-only distributions of $m_{t \bar{t}}$ for the two-b-tag signal regions of the 2016 analysis, with the $\Delta y<1.0$ category on the left and the $\Delta y>1.0$ category on the right. The shaded region corresponds to the combined systematic and statistical uncertainties on the background model.

follows:

$$
\mathcal{L}\left(\beta_{k}\right)=\prod_{i} \frac{\mu_{i} \cdot e^{-\mu_{i}}}{N_{i}^{\text {data } !}} .
$$

For the Poisson parameters, $\beta_{k}$, different choices of prior distributions are used. For the NTMJ estimate and the signal cross section normalization parameters, a flat prior distribution is used. For the other nuisance parameters, a log-normal prior distribution is used. Using these parameters, pseudoexperiments are performed to estimate the $68 \%$ and $95 \%$ CL (1and 2-sigma) expected limit bands. In the pseudoexperiments, the systematic and statistical uncertainties are accounted for through nuisance parameters. These nuisance parameters are randomly varied within their uncertainties, and the posterior is refitted for each individual pseudoexperiment. Through this method, the effect of the shape and normalization uncertainties on the sensitivity of the analysis is evaluated.

Figure 9.0.3 shows the expected cross section limits obtained from the 2016 analysis, using the combination of the six independent signal regions A-F. The dashed line represents the median expected limit, while the green and yellow bands represent the one and two sigma 
bands, respectively, of the expected limits from the set of pseudoexperiments. The red solid line shows the theoretical cross sections curves $-1.3 \times$ leading order (LO) from PYTHIA8 for RS KK gluon and next-to-leading order (NLO) for the $Z^{\prime}$ curves [78]. The expected cross section limits are shown numerically in Tables 9.39 .6 .

As a point of comparison, the limits obtained from combining the 2015 version of this analysis, which did not use PUPPI variables, with the semileptonic channel, both using $2.6 \mathrm{fb}^{-1}$ of data, are shown in Fig. 9.0.4. These plots are similar to those of Fig. 9.0.3, though they have one additional curve. The black solid line shows the observed limit results using the observed data distribution. The expected and observed (2015 only) mass exclusion limits for the 2016 all-hadronic and 2015 analyses are listed in tables 9.1 and 9.2 , respectively. In 2015, no excess of events above the expected background from SM $t \bar{t}$ and nontop multijet events were observed. For all signal hypotheses, the 2016 analysis is expected to extend the limits to higher masses, as compared to the combined 2015 analysis.

\begin{tabular}{l|c}
\multicolumn{2}{c}{ Mass Exclusion Limits } \\
Signal Model & Expected Exclusion Range (TeV) \\
\hline$Z^{\prime}(1 \%$ Width $)$ & $1.0-3.5$ \\
$Z^{\prime}(10 \%$ Width $)$ & $1.0-4.3$ \\
$Z^{\prime}(30 \%$ Width $)$ & $1.0-5.0$ \\
RS Gluon & $1.0-$
\end{tabular}

Table 9.1: Expected exclusion ranges for resonance masses in each of the signal models tested in the 2016 analysis.

\begin{tabular}{lcccccccc} 
& \multicolumn{1}{c}{ Excluded mass ranges $[\mathrm{TeV}]$} \\
Result & $\mathrm{Z}^{\prime}(\Gamma / M=1 \%)$ & \multicolumn{2}{c}{$\mathrm{Z}^{\prime}(\Gamma / M=10 \%)$} & $\mathrm{Z}^{\prime}(\Gamma / M=30 \%)$ & \multicolumn{2}{c}{ RS KK Gluon } \\
\hline Semileptonic & $0.6-2.1$ & $0.6-2.3$ & $0.5-3.5$ & $0.5-3.4$ & $0.5-4.0$ & $0.5-4.0$ & $0.5-2.9$ & $0.5-2.9$ \\
All-hadronic & $1.2-1.8$ & $1.4-1.8$ & $1.0-3.2$ & $1.0-3.5$ & $1.0-3.7$ & $1.0-4.0$ & $1.0-2.6$ & $1.0-2.4$ \\
Combined & $0.6-2.4$ & $0.6-2.5$ & $0.5-3.7$ & $0.5-3.9$ & $0.5-4.0$ & $0.5-4.0$ & $0.5-3.1$ & $0.5-3.3$
\end{tabular}

Table 9.2: Comparison of 2015 mass exclusion results (in $\mathrm{TeV}$ ) for the semileptonic, allhadronic, and combined channels [67. 

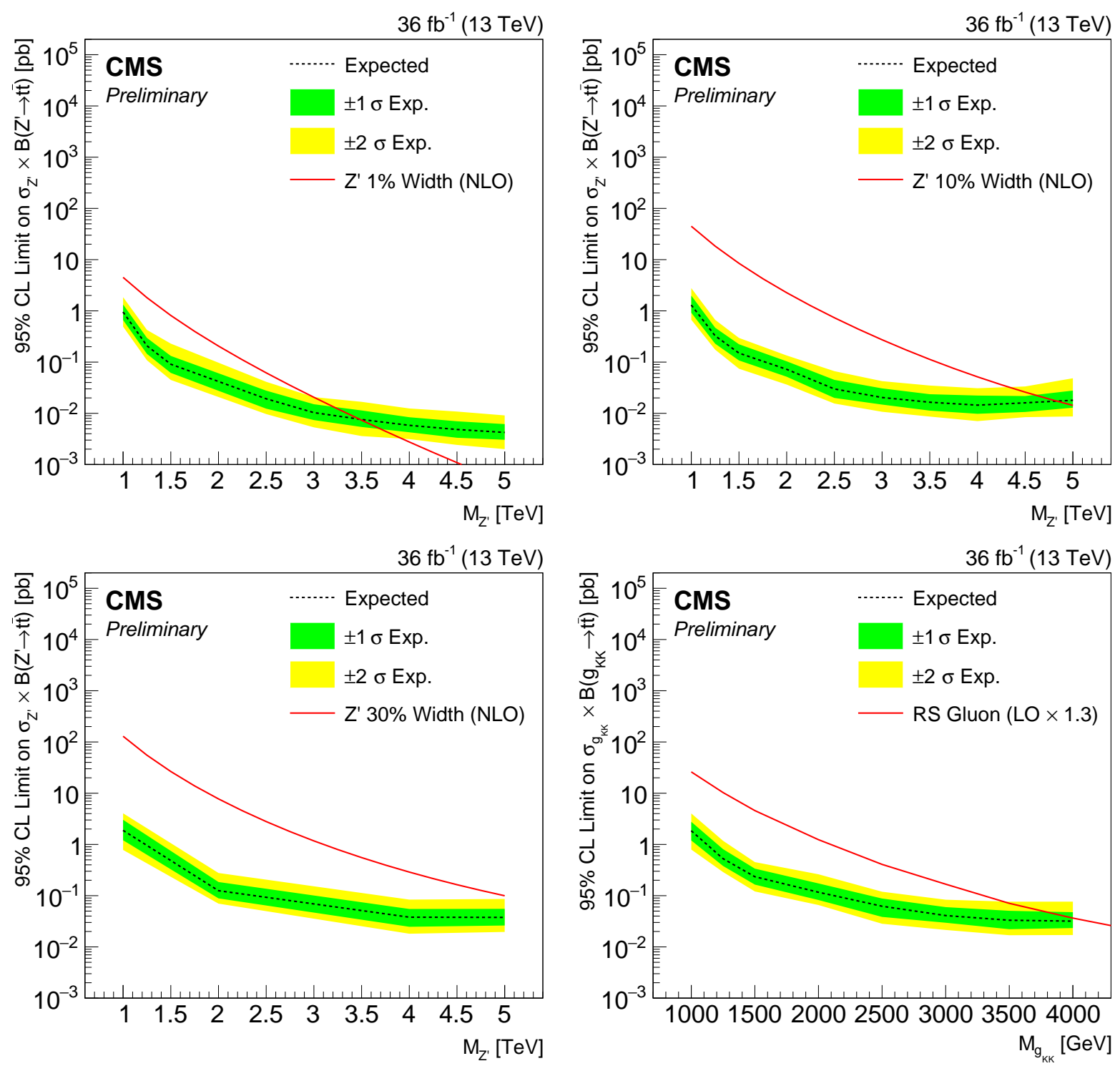

Figure 9.0.3: Expected 2016 95\% CL upper limits on the cross section times branching ratio for the four signal models, as a function of the new heavy particle mass. The four models considered are a $Z^{\prime}$ boson whose width is $1 \%$ of its mass (upper left), a $10 \%$ width $Z^{\prime}$ boson (upper right), a 30\% width $Z^{\prime}$ boson (lower left), and an RS KK gluon (lower right). The black dashed line gives the median expected limits, while the one (two) sigma expected limit band is shown in green (yellow). The red solid line shows the expected theoretical cross section. 

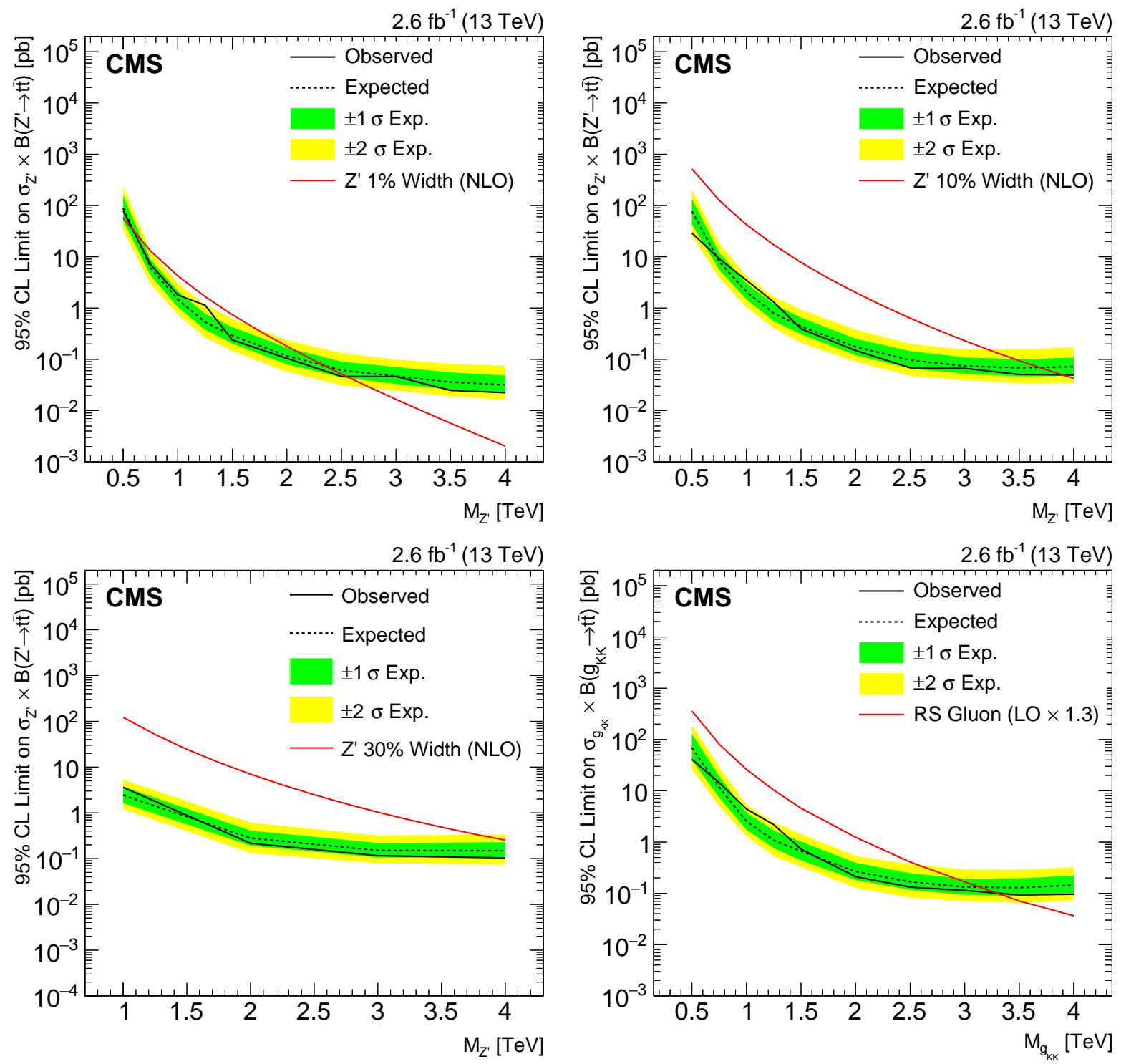

Figure 9.0.4: Observed and expected 2015 95\% CL upper limits on the cross section times branching ratio for the four signal models, as a function of the new heavy particle mass, for the combined semileptonic and all-hadronic channels. The four models considered are a $Z^{\prime}$ boson whose width is $1 \%$ of its mass (upper left), a $10 \%$ width $Z^{\prime}$ boson (upper right), a $30 \%$ width $Z^{\prime}$ boson (lower left), and an RS KK gluon (lower right). The black, solid (dashed) line gives the observed (median expected) limits, while the one (two) sigma expected limit band is shown in green (yellow). The red solid line shows the expected theoretical cross section [67]. 


\begin{tabular}{lccccc}
\multicolumn{7}{c}{$Z^{\prime}$} & $(\mathbf{1 \%}$ Width) Signal Hypothesis \\
Mass $(\mathrm{GeV})$ & \multicolumn{7}{c}{ Expected } & 95\% CL Limits $(\mathrm{pb})$ \\
& $-2 \sigma$ & $-1 \sigma$ & Median & $+1 \sigma$ & $+2 \sigma$ \\
\hline 1000 & 0.49 & 0.66 & $\mathbf{0 . 9 5}$ & 1.3 & 1.8 \\
1250 & 0.11 & 0.14 & $\mathbf{0 . 2 1}$ & 0.3 & 0.42 \\
1500 & 0.045 & 0.062 & $\mathbf{0 . 0 9 1}$ & 0.13 & 0.23 \\
2500 & 0.0097 & 0.012 & $\mathbf{0 . 0 1 9}$ & 0.028 & 0.041 \\
3000 & 0.0053 & 0.0074 & $\mathbf{0 . 0 1}$ & 0.015 & 0.021 \\
3500 & 0.0036 & 0.0054 & $\mathbf{0 . 0 0 7 6}$ & 0.011 & 0.017 \\
4000 & 0.0031 & 0.0043 & $\mathbf{0 . 0 0 5 8}$ & 0.0084 & 0.012 \\
4500 & 0.0024 & 0.0033 & $\mathbf{0 . 0 0 4 8}$ & 0.007 & 0.011 \\
5000 & 0.002 & 0.003 & $\mathbf{0 . 0 0 4 2}$ & 0.0062 & 0.0091
\end{tabular}

Table 9.3: Table of expected 2016 95\% CL cross section limits, for the narrow (1\% width) $Z^{\prime}$ signal hypothesis.

\begin{tabular}{lccccc}
\multicolumn{7}{c}{$Z^{\prime}(\mathbf{1 0 \%}$ Width) Signal Hypothesis } \\
Mass $(\mathrm{GeV})$ & \multicolumn{7}{c}{ Expected } & 95\% CL Limits $(\mathrm{pb})$ \\
& $-2 \sigma$ & $-1 \sigma$ & Median & $+1 \sigma$ & $+2 \sigma$ \\
\hline 1000 & 0.67 & 0.92 & $\mathbf{1 . 3}$ & 2 & 2.8 \\
1250 & 0.18 & 0.23 & $\mathbf{0 . 3 3}$ & 0.47 & 0.67 \\
1500 & 0.074 & 0.11 & $\mathbf{0 . 1 5}$ & 0.22 & 0.3 \\
2000 & 0.036 & 0.052 & $\mathbf{0 . 0 7 2}$ & 0.1 & 0.13 \\
2500 & 0.016 & 0.02 & $\mathbf{0 . 0 3}$ & 0.045 & 0.067 \\
3000 & 0.011 & 0.015 & $\mathbf{0 . 0 2}$ & 0.031 & 0.042 \\
3500 & 0.0086 & 0.011 & $\mathbf{0 . 0 1 6}$ & 0.024 & 0.035 \\
4000 & 0.007 & 0.0098 & $\mathbf{0 . 0 1 4}$ & 0.022 & 0.031 \\
4500 & 0.0084 & 0.011 & $\mathbf{0 . 0 1 6}$ & 0.022 & 0.033 \\
5000 & 0.0087 & 0.013 & $\mathbf{0 . 0 1 8}$ & 0.028 & 0.048
\end{tabular}

Table 9.4: Table of expected 2016 95\% CL cross section limits, for the wide (10\% width) $Z^{\prime}$ signal hypothesis.

\begin{tabular}{cccccc}
\multicolumn{2}{c}{$Z^{\prime}(\mathbf{3 0 \%}$ Width) Signal Hypothesis } \\
Mass (GeV) & \multicolumn{4}{c}{ Expected $95 \%$ CL Limits $(\mathrm{pb})$} \\
& $-2 \sigma$ & $-1 \sigma$ & Median & $+1 \sigma$ & $+2 \sigma$ \\
\hline 1000 & 0.78 & 1.2 & $\mathbf{1 . 9}$ & 3 & 4.1 \\
2000 & 0.07 & 0.089 & $\mathbf{0 . 1 3}$ & 0.18 & 0.28 \\
4000 & 0.018 & 0.025 & $\mathbf{0 . 0 3 8}$ & 0.055 & 0.084 \\
5000 & 0.02 & 0.026 & $\mathbf{0 . 0 3 8}$ & 0.055 & 0.086
\end{tabular}

Table 9.5: Table of expected 2016 95\% CL cross section limits, for the extra wide (30\% width) $Z^{\prime}$ signal hypothesis. 


\begin{tabular}{lccccc}
\multicolumn{7}{c}{ RS Gluon Signal Hypothesis } \\
Mass $(\mathrm{GeV})$ & \multicolumn{7}{c}{ Expected } & $95 \%$ CL Limits $(\mathrm{pb})$ \\
& $-2 \sigma$ & $-1 \sigma$ & Median & $+1 \sigma$ & $+2 \sigma$ \\
\hline 1000 & 0.79 & 1.2 & $\mathbf{1 . 8}$ & 2.8 & 4 \\
1250 & 0.29 & 0.38 & $\mathbf{0 . 5 3}$ & 0.81 & 1.2 \\
1500 & 0.12 & 0.16 & $\mathbf{0 . 2 3}$ & 0.34 & 0.45 \\
2000 & 0.066 & 0.082 & $\mathbf{0 . 1 2}$ & 0.17 & 0.26 \\
2500 & 0.028 & 0.039 & $\mathbf{0 . 0 6 2}$ & 0.088 & 0.12 \\
3000 & 0.021 & 0.03 & $\mathbf{0 . 0 4 1}$ & 0.06 & 0.083 \\
3500 & 0.017 & 0.022 & $\mathbf{0 . 0 3 3}$ & 0.051 & 0.076 \\
4000 & 0.017 & 0.023 & $\mathbf{0 . 0 3 2}$ & 0.048 & 0.076
\end{tabular}

Table 9.6: Table of expected 2016 95\% CL cross section limits, for the RS Gluon signal hypothesis. 


\section{Chapter 10}

\section{Conclusions}

A search for top-quark-pair resonances in the all-hadronic channel has been performed using $\sqrt{s}=13 \mathrm{TeV}$ data from the LHC Run 2 taken in 2016. The search uses a well understood top tagging algorithm, optimized for Run 2 analyses, using the softdrop mass and n-subjettiness jet substructure variables. For the first time, jet top tagging variables are calculated using PUPPI inputs. The nontop multijet background is estimated using a data-driven top-mistag rate measurement. In 2015, the the combined semileptonic and all-hadronic analysis observed no excess above the Standard Model expectation, and limits were set on the signal production cross section for the four signal models. The 2016 expected limits show that the 2016 allhadronic analyses is expected to exceed the combined 2015 semileptonic and all-hadronic analysis in sensitivity. In the future, this analysis will be combined with both the semileptonic and dileptonic $Z^{\prime}$ search channels, providing the most sensitive result to date. 


\section{Appendix A}

\section{Muon Hardware Projects}

\section{A.1 CSC Hardware}

As part of the CMS experiment, I was involved in detector maintenance, operations, and upgrades of the CSC and GEM subsystems. My first project consisted of testing refurbished CSC low voltage monitoring boards (LVMBs), which monitor the output voltages and currents of the boards that distribute the low voltage to the CSC on-board electronics. During Run I of the LHC, it was found that some of the CSC ME1/1 LVMBs were missing a capacitor, which caused them to malfunction when they reached temperatures above $40^{\circ} \mathrm{C}$. During LS1, the capacitors were replaced, and the boards' functionality at high temperatures was verified. Figure A.1.1 shows me monitoring the operation of an LVMB while using a hair dryer to raise its temperature to $40^{\circ}+\mathrm{C}$.

My other main role in the CMS CSC hardware group was that of a detector-on-call (DOC) shifter. For a week at a time, the CSC DOC is responsible for the operation of the CMS CSC subsystem. This includes attending daily run meetings at the CMS site ("Point 5"), performing daily CSC maintenance activities, and debugging any problems that might arise. The DOC is the first point of contact for any CSC-related actions in CMS and therefore must coordinate with the other CMS shifters and CSC experts. Figure A.1.2 shows me working 


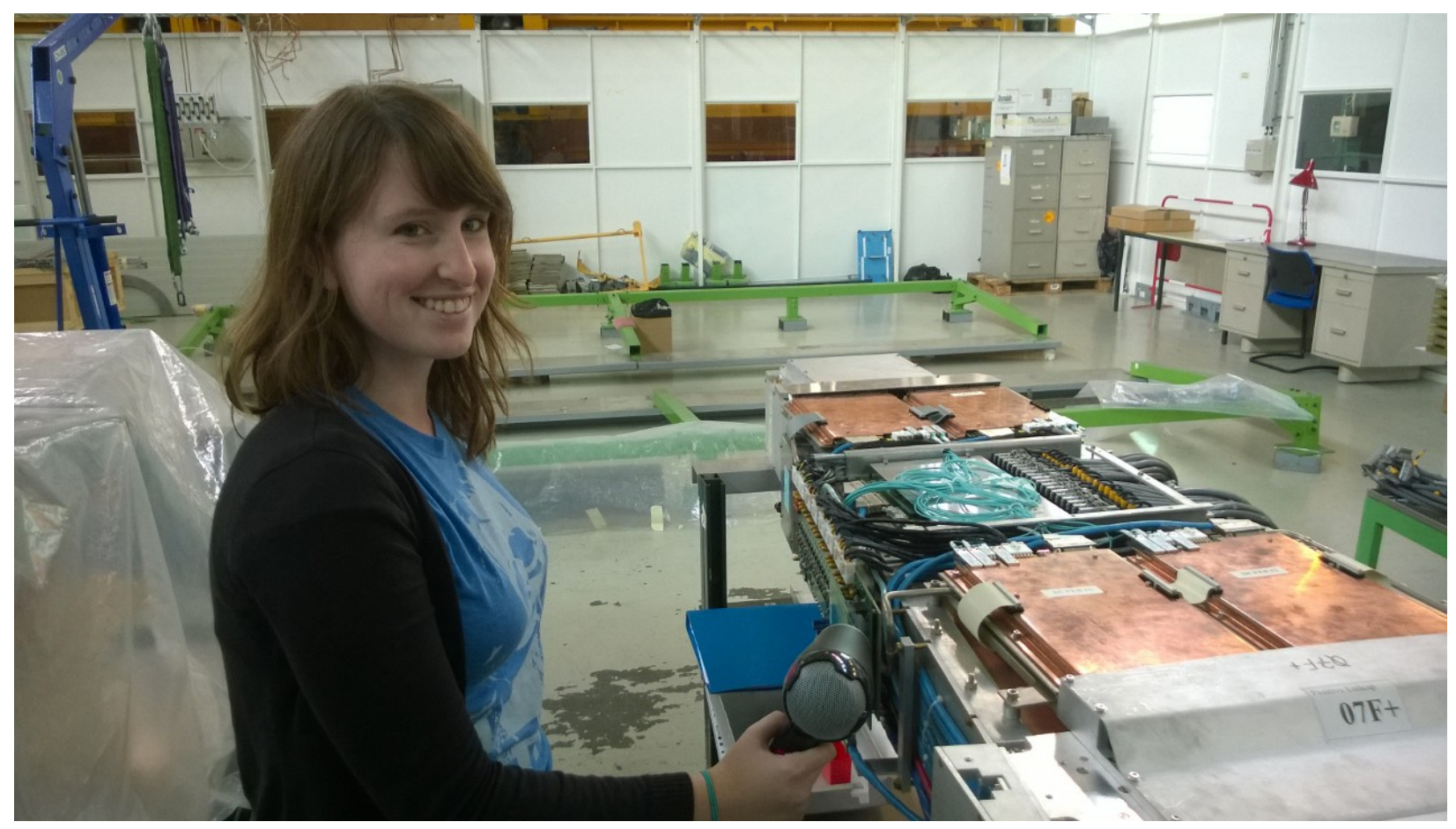

Figure A.1.1: LVMB Testing.

at the CSC DOC desk in the CMS control room. On the left, I am working with the CSC operations manager to debug noise seen on two ME1/1 chambers while the CMS magnet was ramped up to $1 \mathrm{~T}$. On the right, I am performing a daily CSC firmware check while a TV crew films a skype conversation between the CMS Spokesperson and one of their colleagues.

\section{A.2 GEM Hardware}

\section{A.2.1 VFAT2 Testing}

In June 2013, CMS approved the installation of a set of GEM test chambers (known as the GEM demonstrator) into the muon endcap YE-1 in order to test the capability of the new subsystem in LHC beam conditions and to test the integration of the GE1/1 chambers into the trigger [31]. During EYETS 2016-2017, five prototype GEMINI were installed in the slots shown in Fig. A.2.1. At CERN, I was responsible for coordinating the production and 


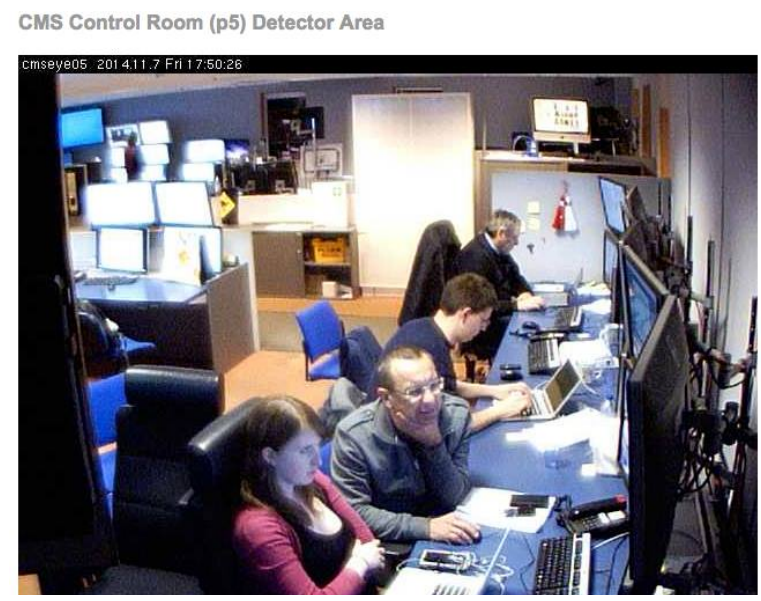

CMS Control Room (p5) Detector Area

Figure A.1.2: Working at the CSC shifter desk.

testing of the VFAT2 readout chips used in the prototype GEM chambers. First, the printed circuit boards (PCB) were shipped to CERN from Lappeenranta University of Technology (Lappeenranta, Finland). I picked them up, took them to be cleaned and then to be partially bonded to the ASIC chip. After this first bonding step, I took them to the test stand in building 904 to check that none of the chips or PCBs were faulty. Specifically, I tested that they could communicate with the GEM DAQ system, which uses the Inter-Integrated Circuit $\left(\mathrm{I}^{2} \mathrm{C}\right)$ protocol to communicate via the on-chamber Optohybrid $(\mathrm{OH})$, the GEM FPGA board [79]. Figure A.2.2 shows me testing some VFAT2 chips, which are plugged into the GEM electronics board (GEB), not a full detector.

After the first stage of testing, I took the working VFAT2s to have the rest of the bonding done. Figure A.2.3 shows a fully bonded VFAT2 chip. I then took them back to re-test their communication and calibrate the chips. As explained in Section 3.2.6, each VFAT2 reads out 128 of the GEM strips. An important part of chip calibration is ensuring that each channel has the same response to the same input charge. To do this, I used the GEM web DAQ application and the onboard electronics - the tests were not performed in beam conditions. First, I set the VFAT2 readout threshold, the value above which charge on the chip is read out as a signal event. For a given VFAT2, I started by setting the threshold to 0, i.e. the value at which any amount of charge on the strips is recorded as signal. Then, I incrementally 


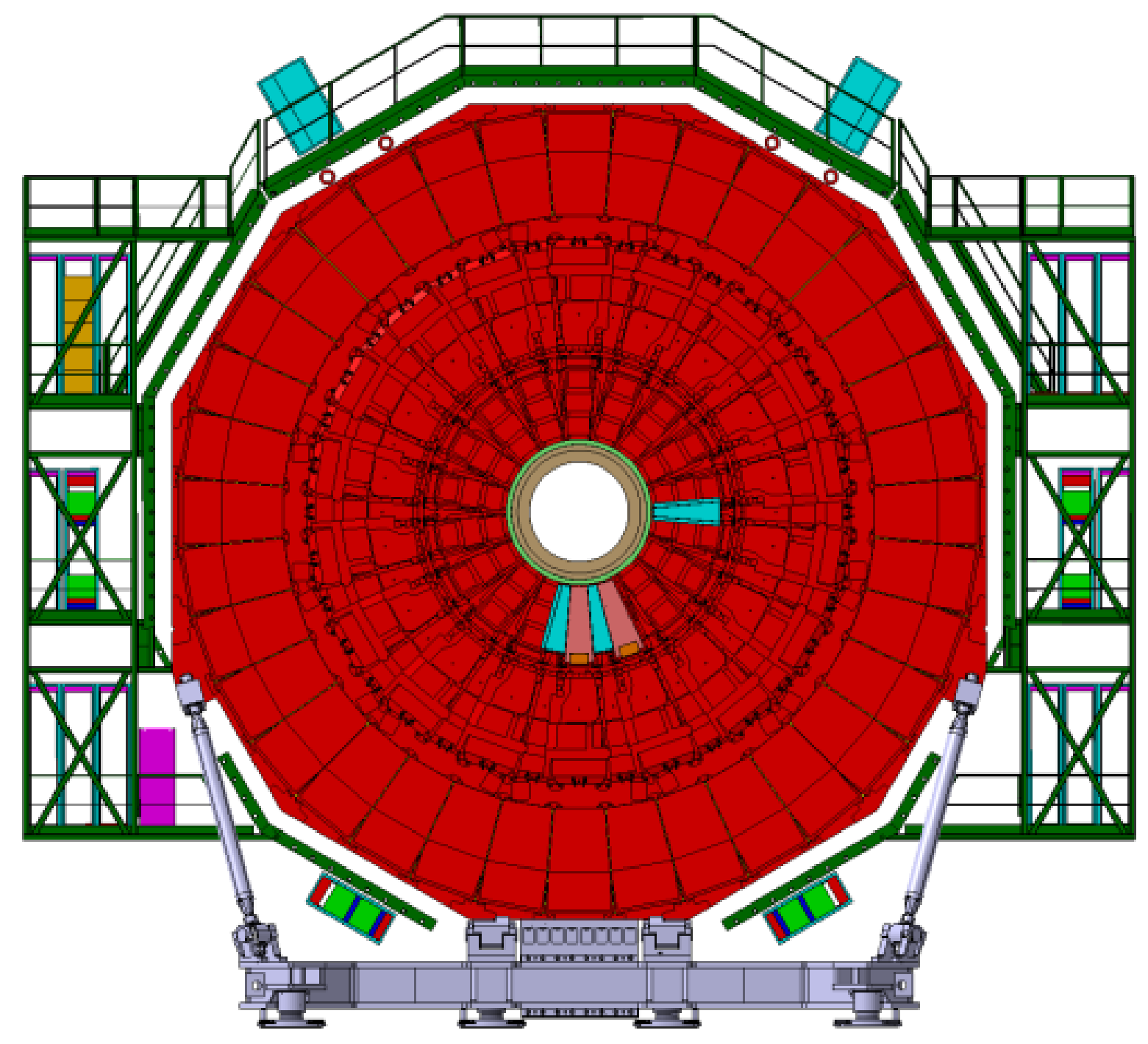

Figure A.2.1: The five slots in which the GEM demonstrator chambers were placed. 


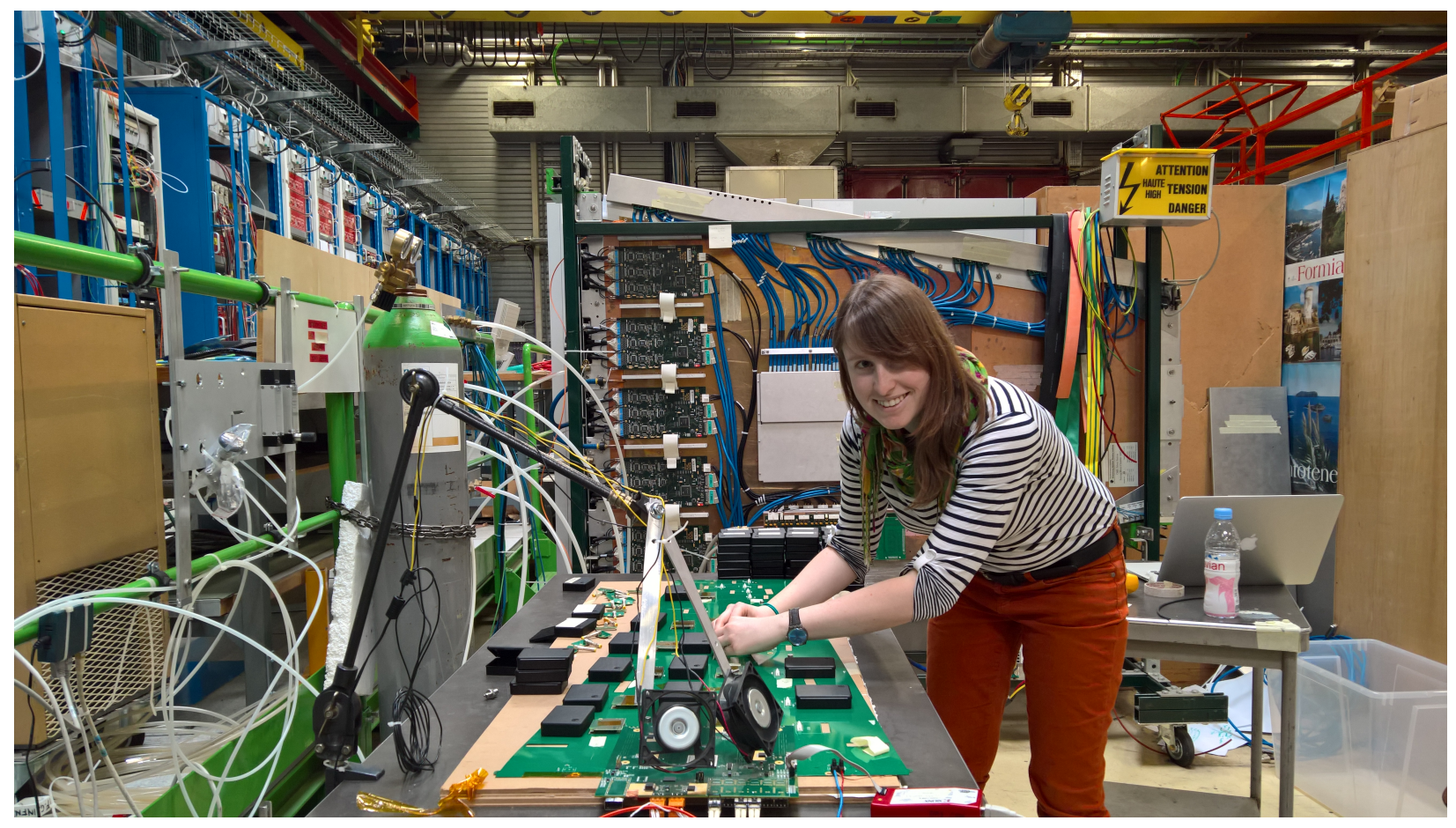

Figure A.2.2: Testing VFAT2s.

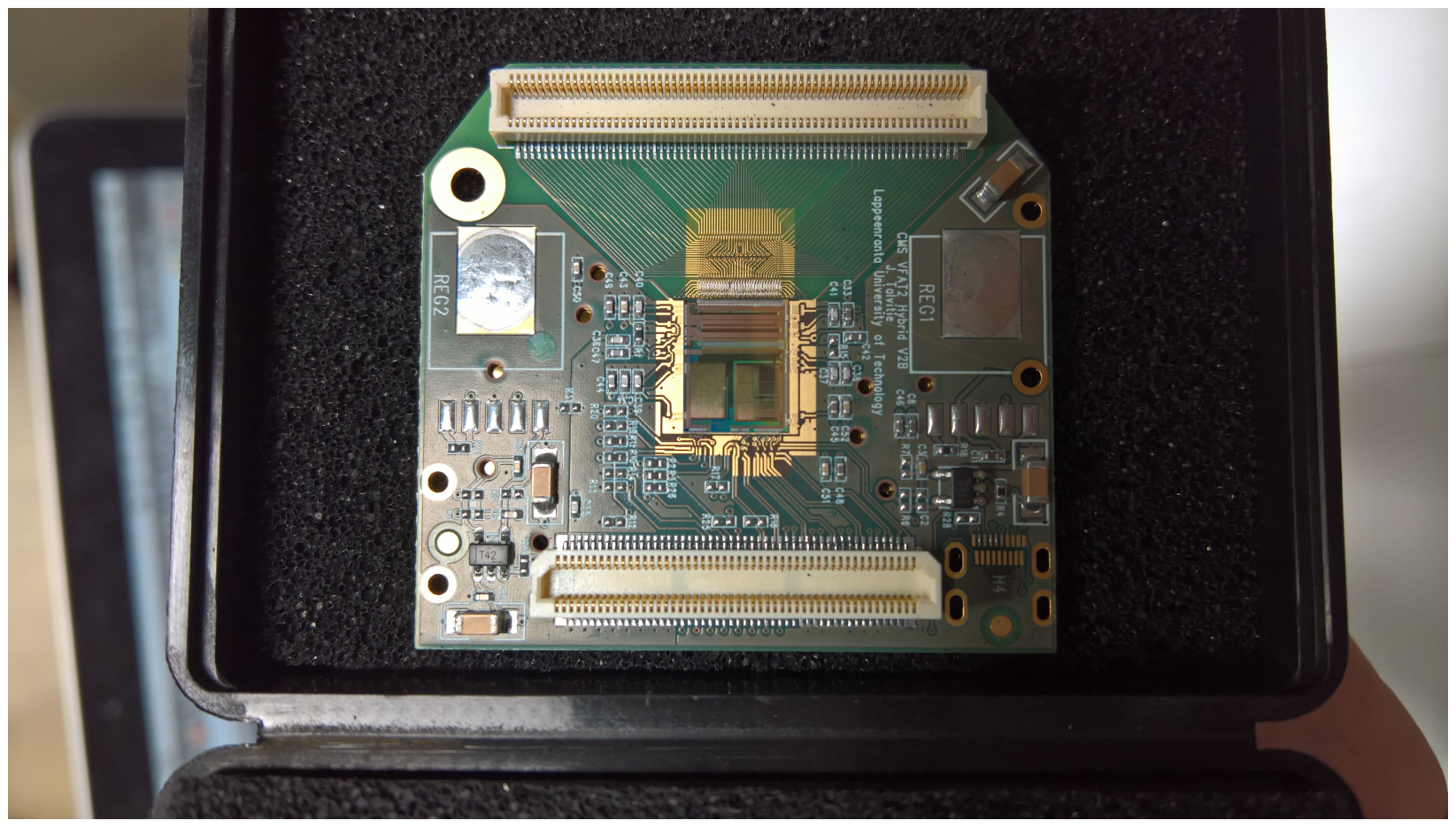

Figure A.2.3: A VFAT2 chip. 
raised the threshold and recorded the percentage of events displaying a hit, measuring the electronics noise of the system. The lowest threshold value that yielded minimal noise was then chosen.

After setting the threshold for a VFAT2 chip, its individual channels were calibrated. A charge pulse was injected by the onboard electronics, and an "s-curve" scan was performed for each channel. The results of this scan for one VFAT2 chip is shown on the left in Fig. A.2.4. which shows the readout efficiency of each channel/strip as a function of injected charge. At this initial stage, the response was not consistent across all channels. Therefore, the thresholds of each channel needed to be finely tuned. This was done by adjusting TrimDAC register for each channel to its minimum and maximum value and performing an s-curve scan at both points. Histograms of the calibration pulse turn-on charge (50\% efficiency) were plotted, and the average value was found. The TrimDAC registers for each channel were set so that they would have $50 \%$ readout efficiency at this average calibration pulse charge. The result is shown in Fig. A.2.4. Indeed, a consistent response is seen across all channels, especially at 50\% efficiency. Therefore, a muon with a given energy will cause a consistent response across the whole chip.

In addition to the 240 VFAT2 chips needed for the GEM demonstrator chambers, more were required for test stands and backups. In total, over 400 chips were commissioned. For the full GE1/1 installation, a customized VFAT3 chip will be used [31].

\section{A.2.2 EYETS}

The final assembly of the GEM demonstrator chambers was completed by the end of 2016 . In January 2017, they were installed in the slots shown in Fig. A.2.1. After the chambers were installed, I helped with the installation of the services and helped ready the database and calibration code for commissioning of the system. Figure A.2.5 shows me connecting the low voltage power supply, while Fig. A.2.6 shows me connecting trigger fibers between a CSC and GEM chamber. Each chamber has a pair of receiving (RX) and transmitting (TX) fibers for 

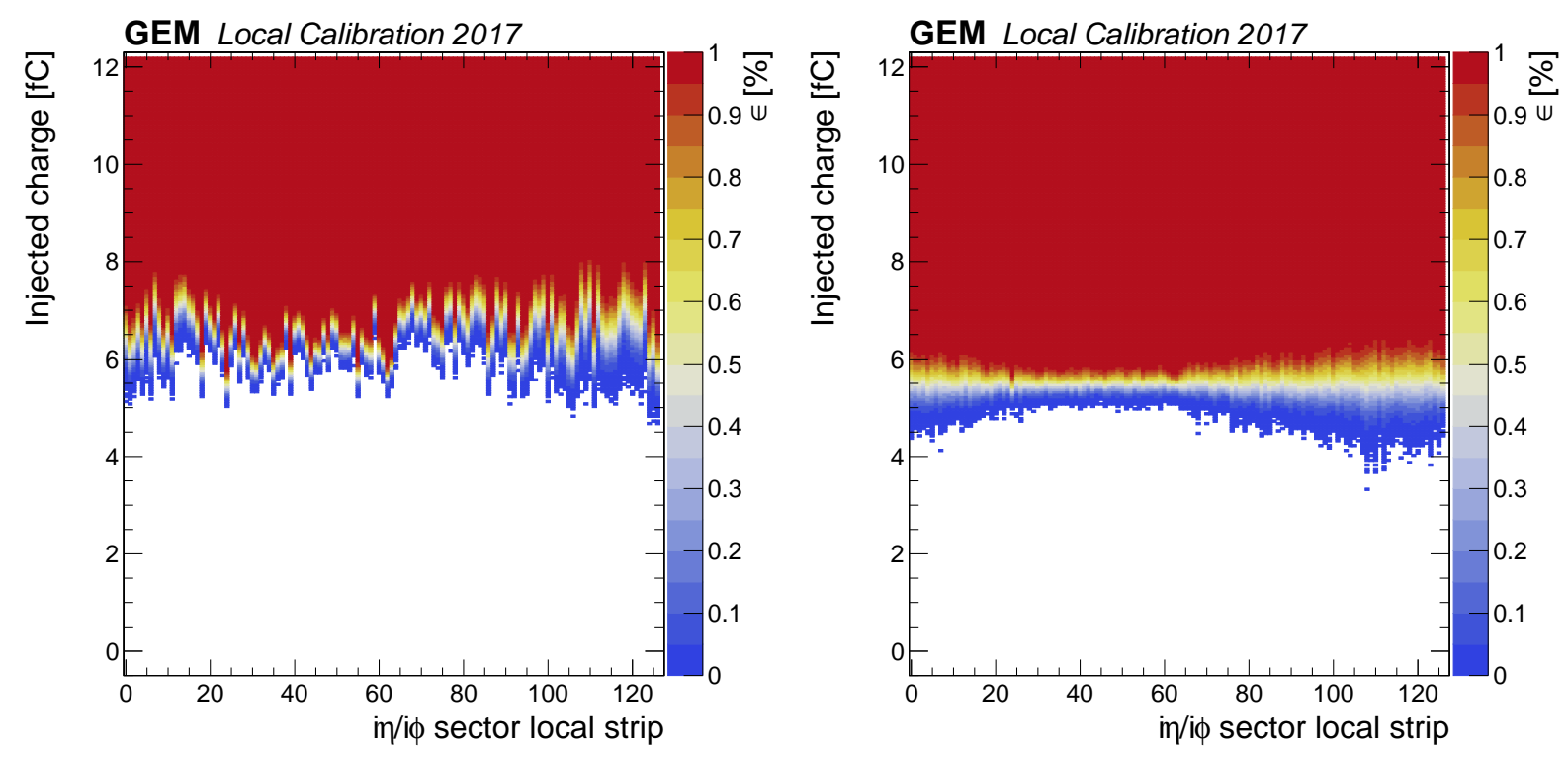

Figure A.2.4: The readout efficiency of the 128 channels of a single VFAT2 chip before (left) and after (right) calibration.

data acquisition (DAQ), trigger (TRG), and the gigabit transceiver (GBTX), which delivers the global system clock reference [31]. The fibers were routed from the detectors in the CMS cavern to an electronics rack in the CMS Underground Service Cavern (USC), shown in Fig. A.2.7. More fibers connected those coming from the CMS cavern (routed through the $\mathrm{OH}$ patch panel $(\mathrm{PP})$ ) to those coming from the DAQ, TRG, and GBTX systems (routed through the CTP7 PP). I documented the fiber mapping in Fig. A.2.8. 


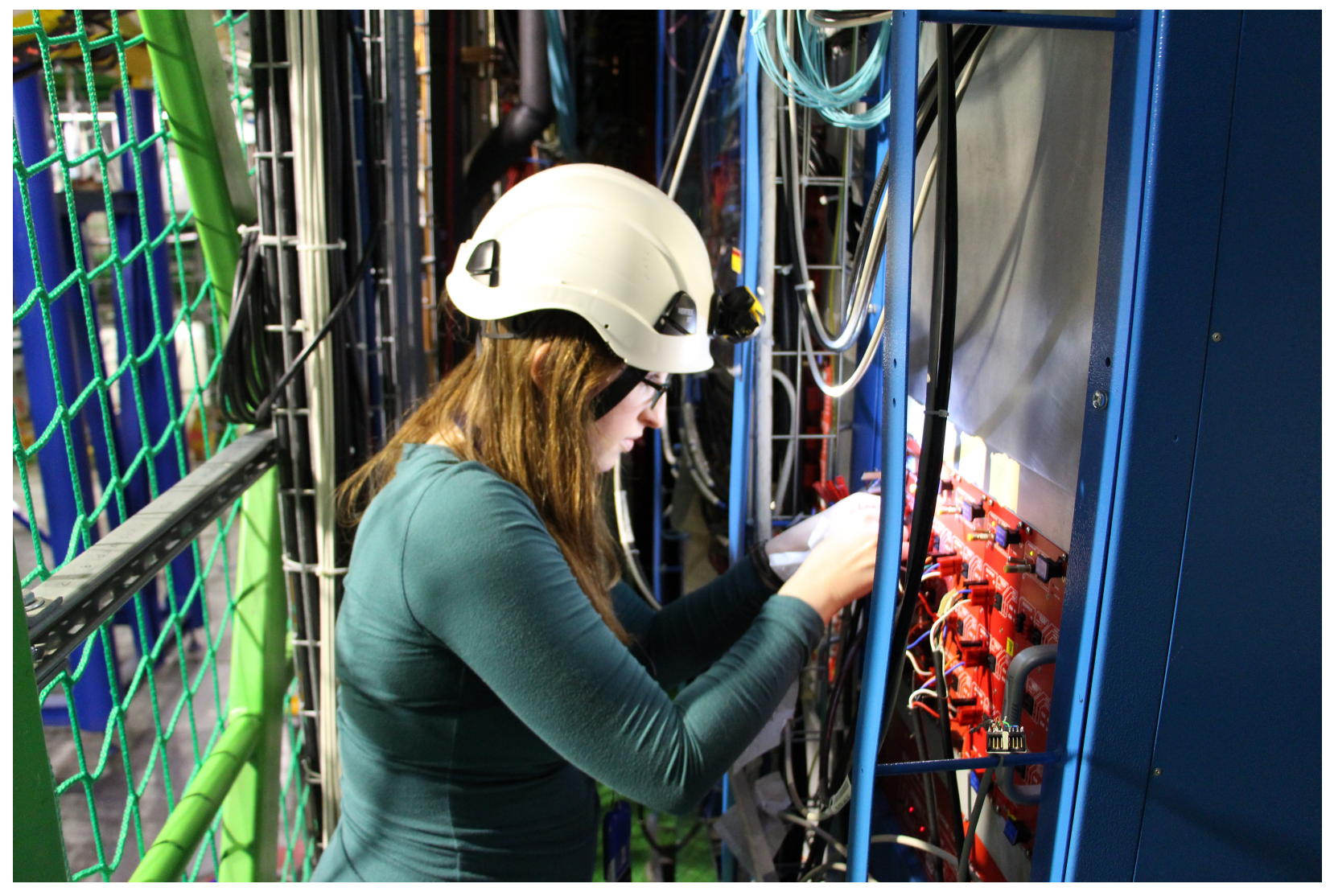

Figure A.2.5: Connecting the low voltage power supply. 


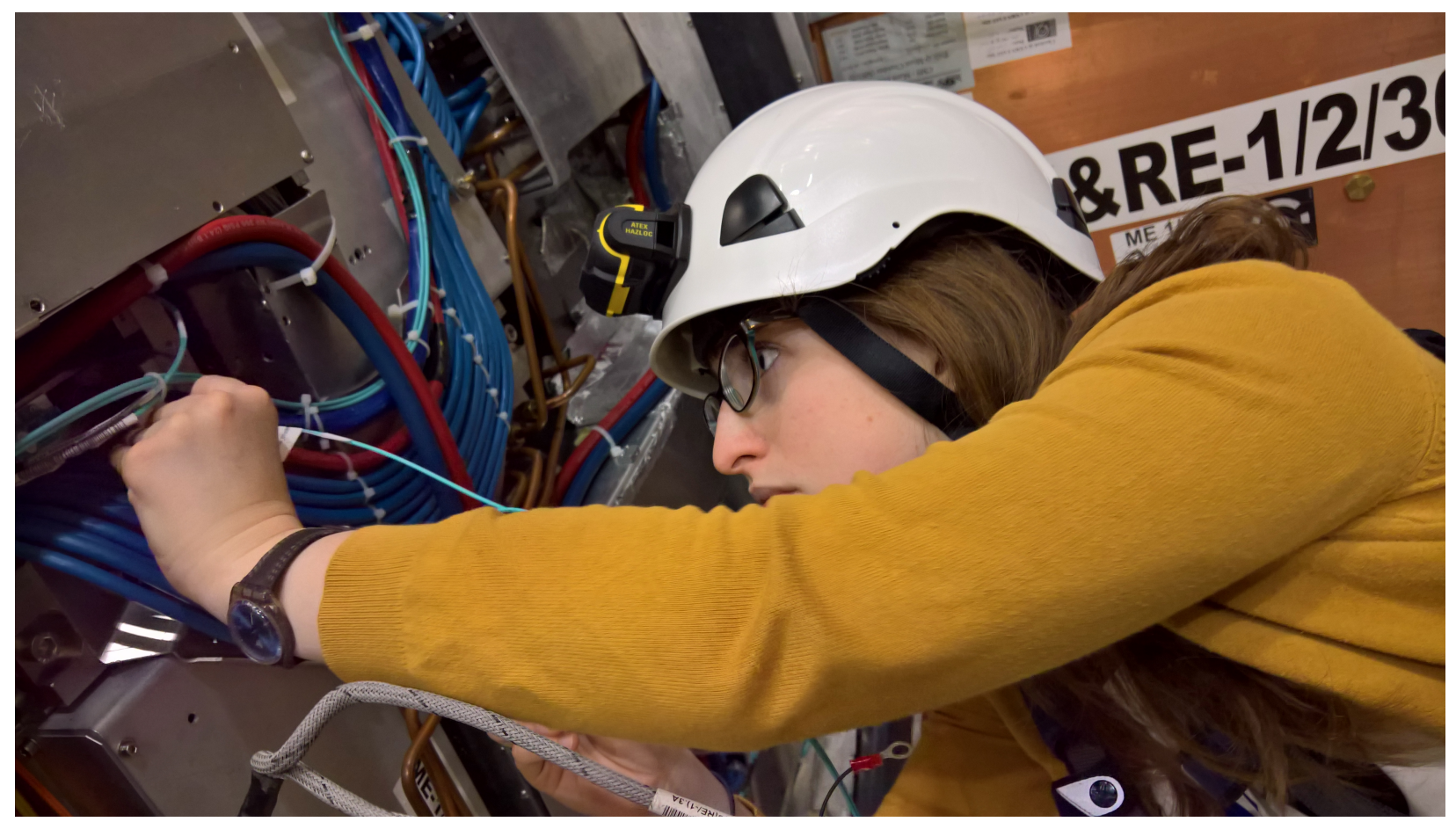

Figure A.2.6: Connecting trigger fibers between CSC and GEM chambers.

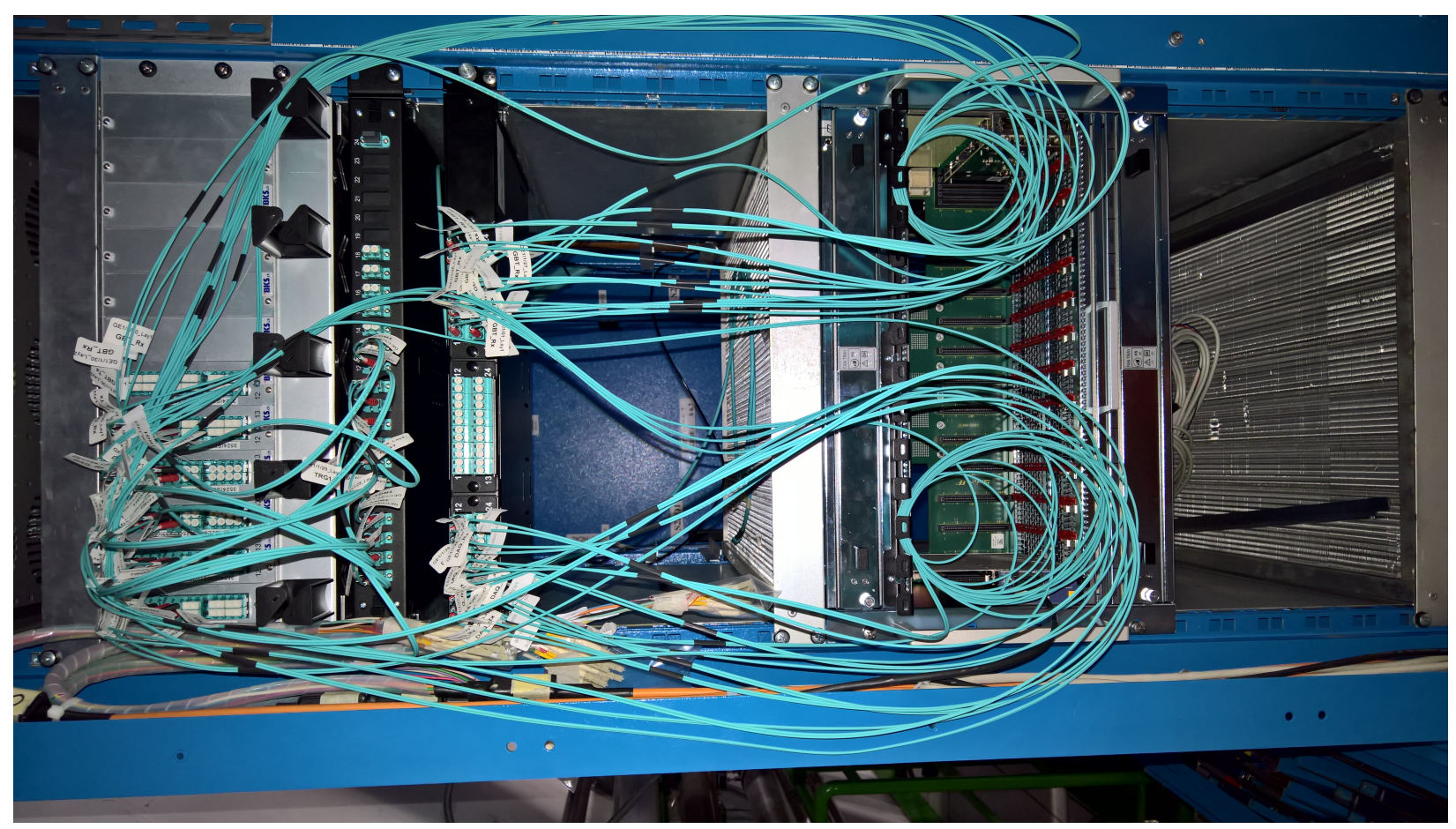

Figure A.2.7: GEM electronics rack in USC. 

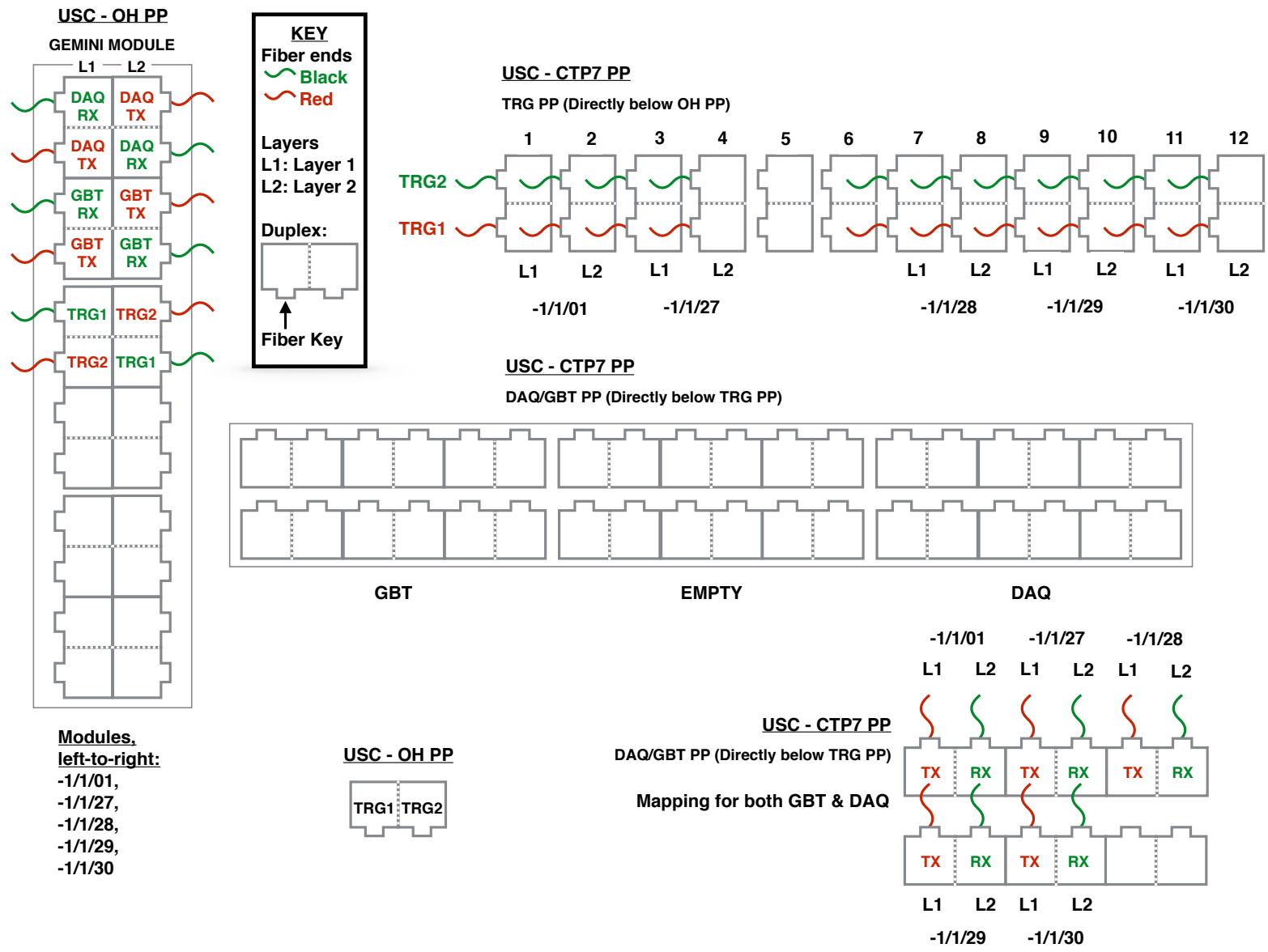

Figure A.2.8: GEM fiber mapping. 


\section{Appendix B}

\section{Additional Kinematic Plots}

This section provides additional kinematic plots comparing data and simulation used in the $Z^{\prime}$ analysis. Of the two QCD samples, the one both simulated and showered with PYTHIA8 shows better agreement with the data, so it is used in the background estimate closure tests. 

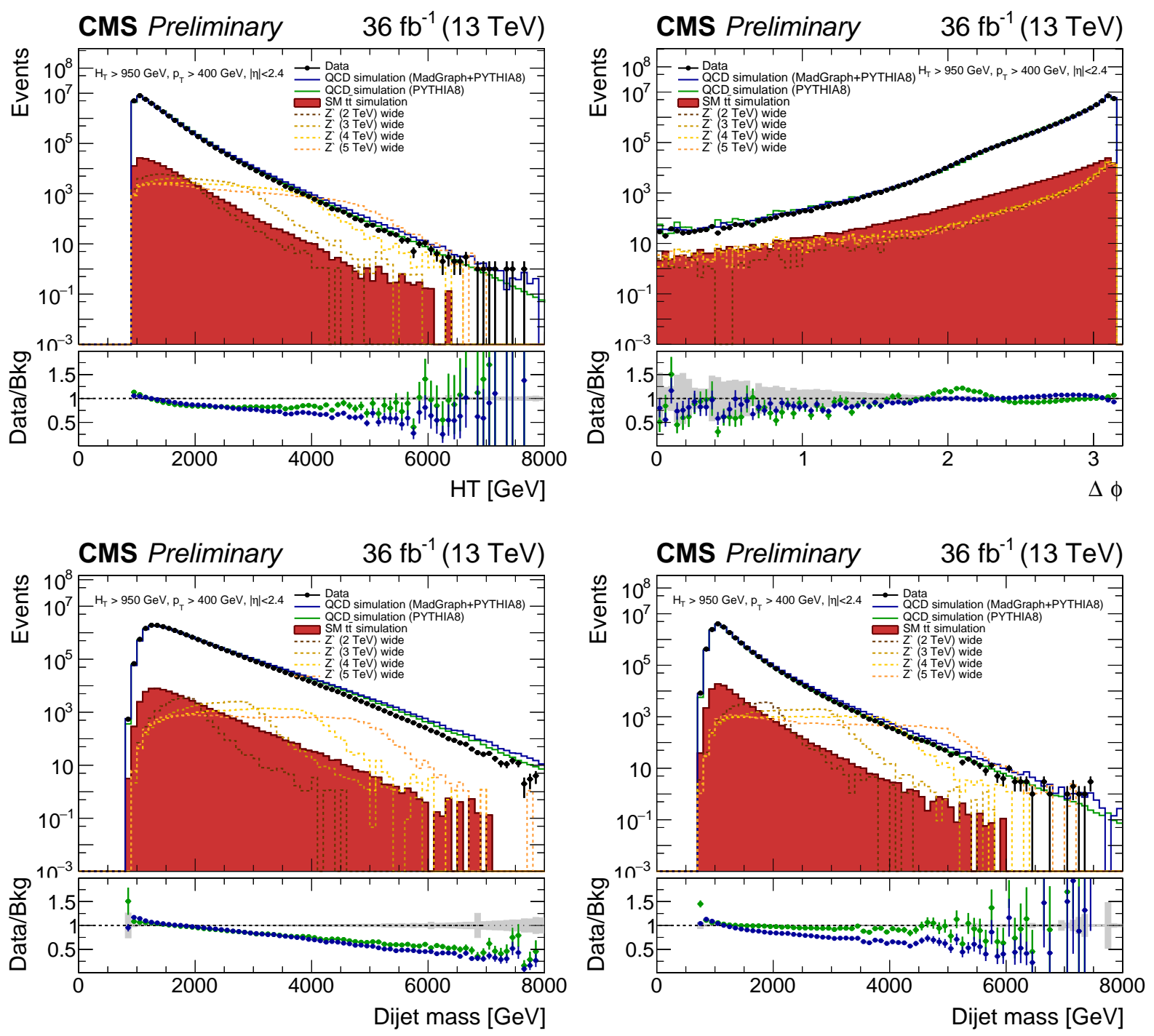

Figure B.0.1: Event kinematic plots after preselection: (a) HT, (b) $\Delta \phi$ (all preselection cuts except for the cut on $\Delta \phi)$, (c) Dijet mass $(\Delta Y>1.0)$, (d) Dijet mass $(\Delta Y<1.0)$. 


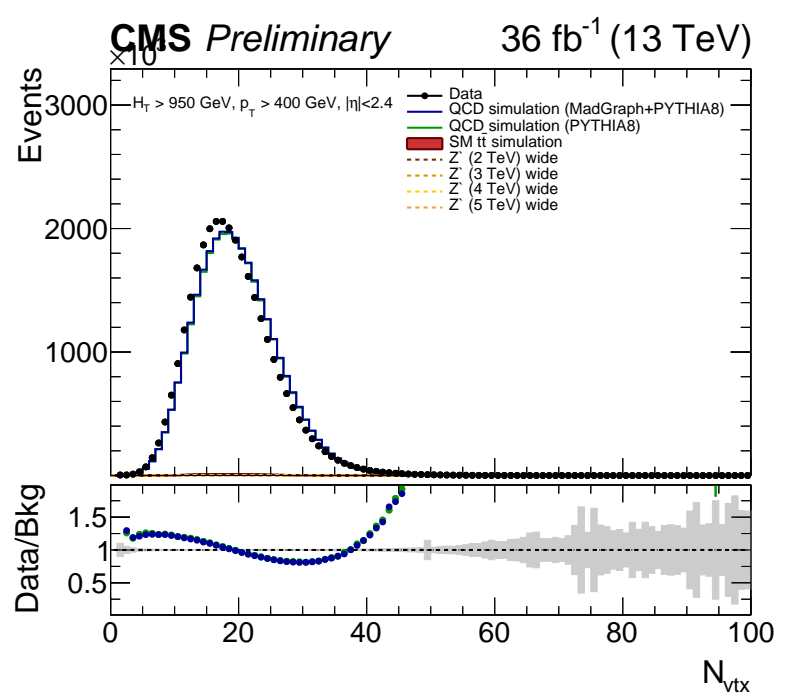

Figure B.0.2: Number of primary vertices
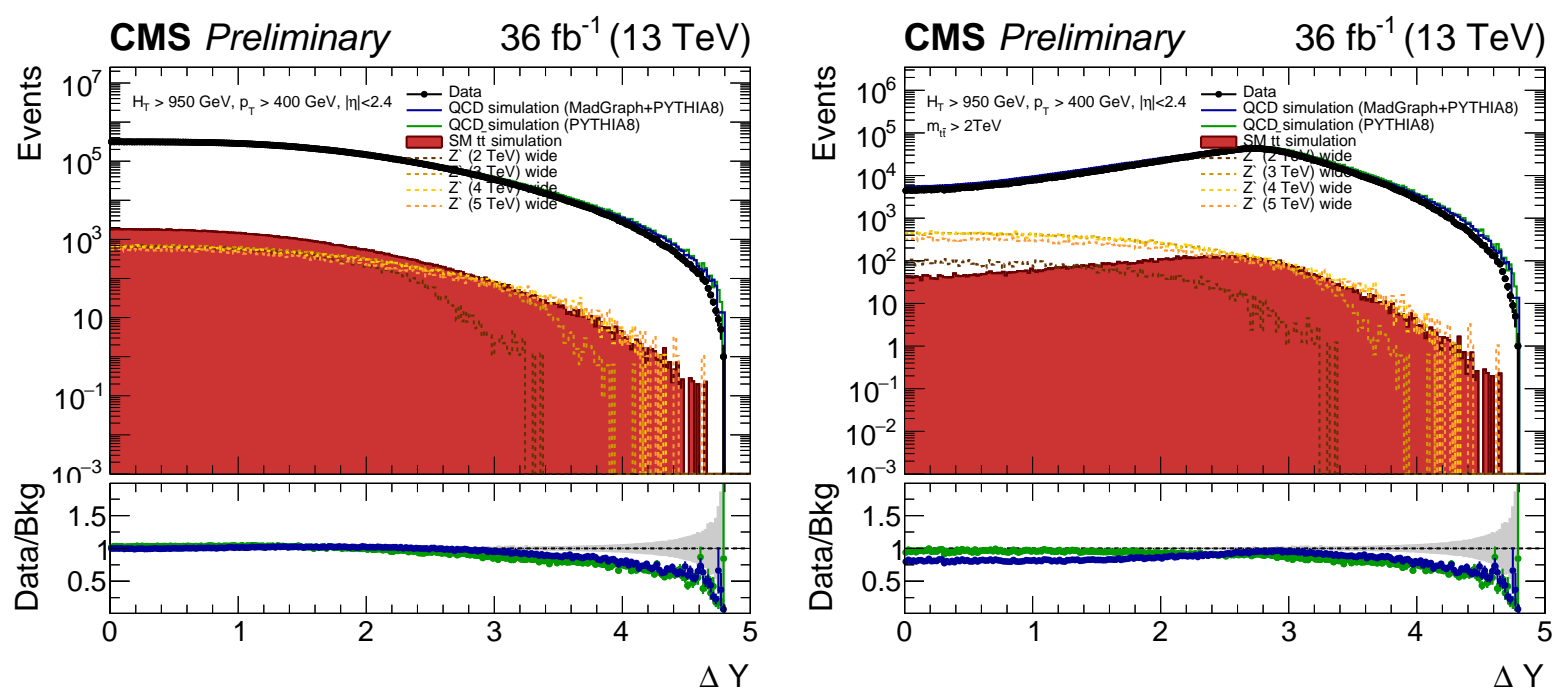

Figure B.0.3: Event kinematic plots after preselection: (a) $\Delta \mathrm{Y}$, (b) $\Delta \mathrm{Y}\left(m_{t t}>2 \mathrm{TeV}\right)$. 

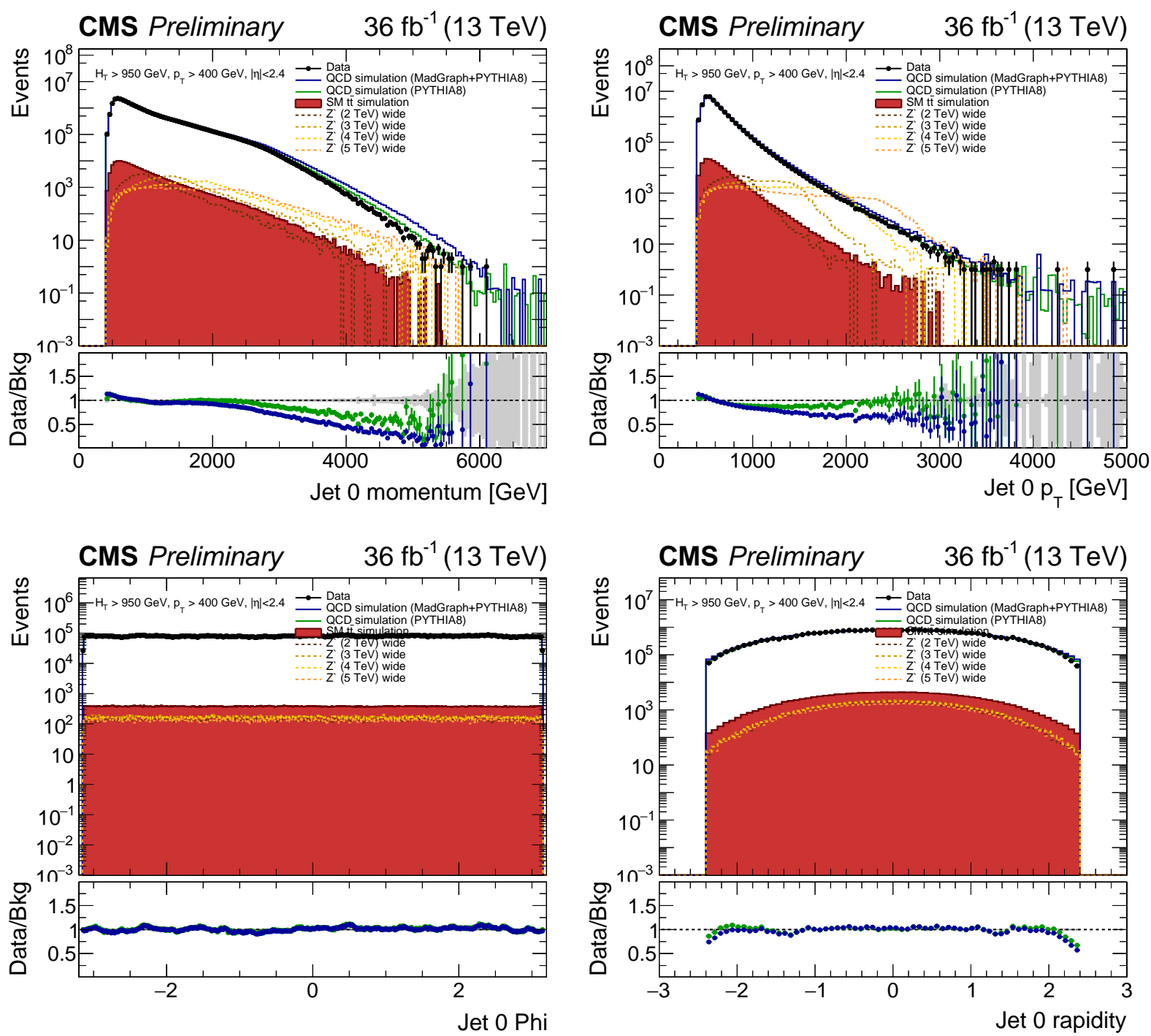

Figure B.0.4: Leading jet kinematic plots after preselection: (a) Jet 0 momentum, (b) Jet $0 p_{T}$, (c) Jet $0 \phi$, (d) Jet 0 rapidity. 

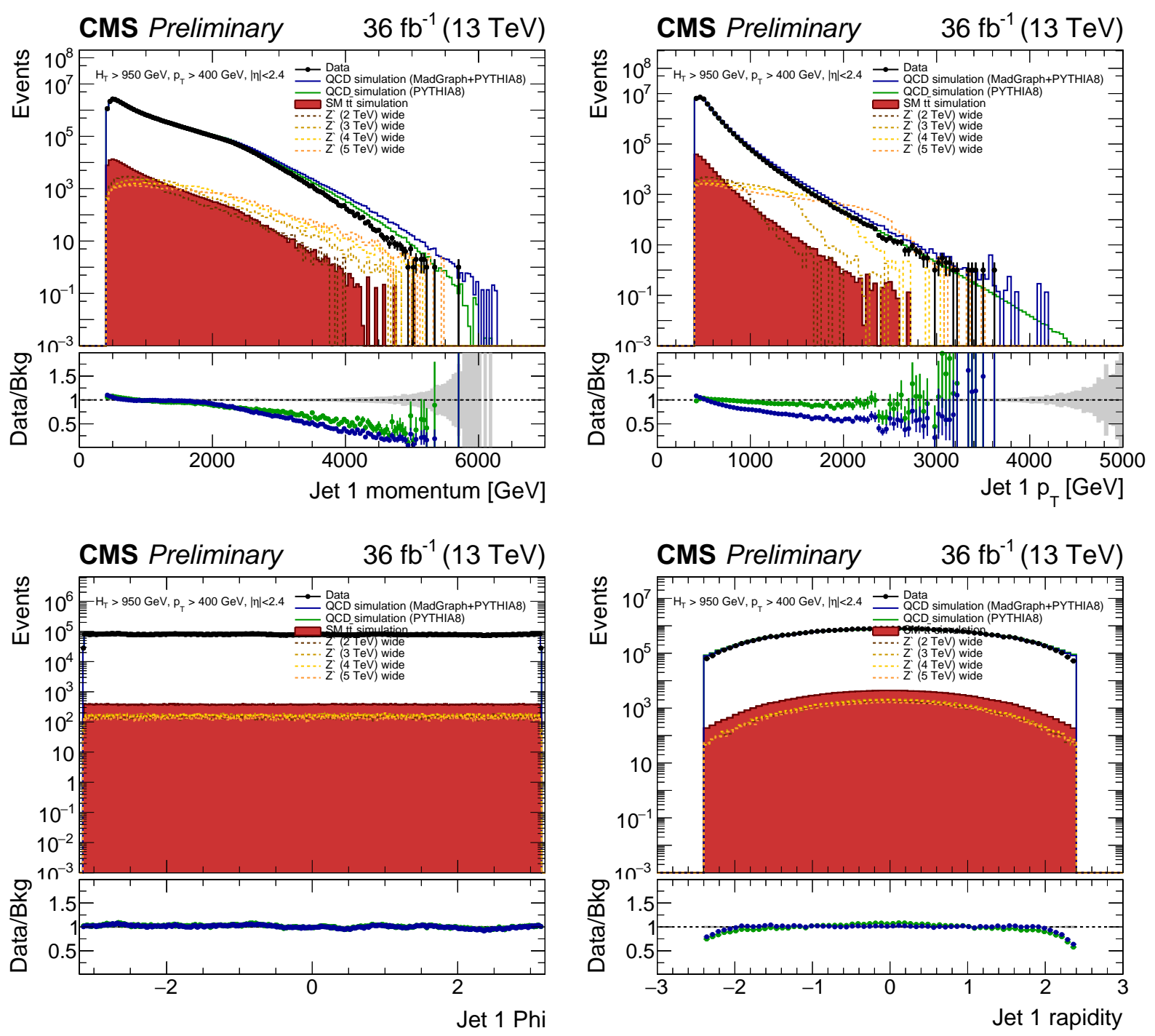

Figure B.0.5: Second leading jet kinematic plots after preselection: (a) Jet 1 momentum, (b) Jet $1 p_{T}$, (c) Jet $1 \phi$, (d) Jet 1 rapidity. 

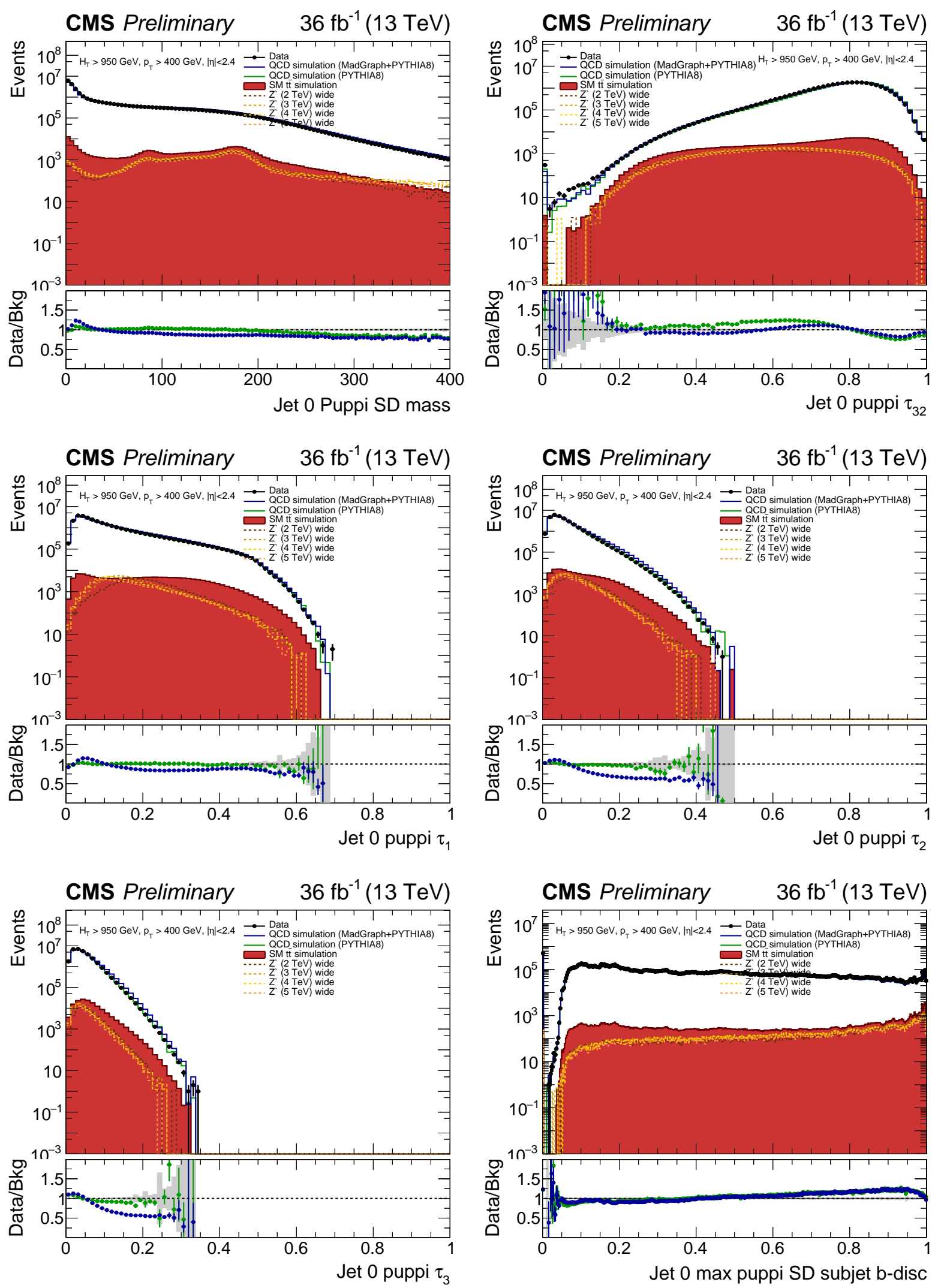

Figure B.0.6: Jet 0 tagging variables after preselection. 

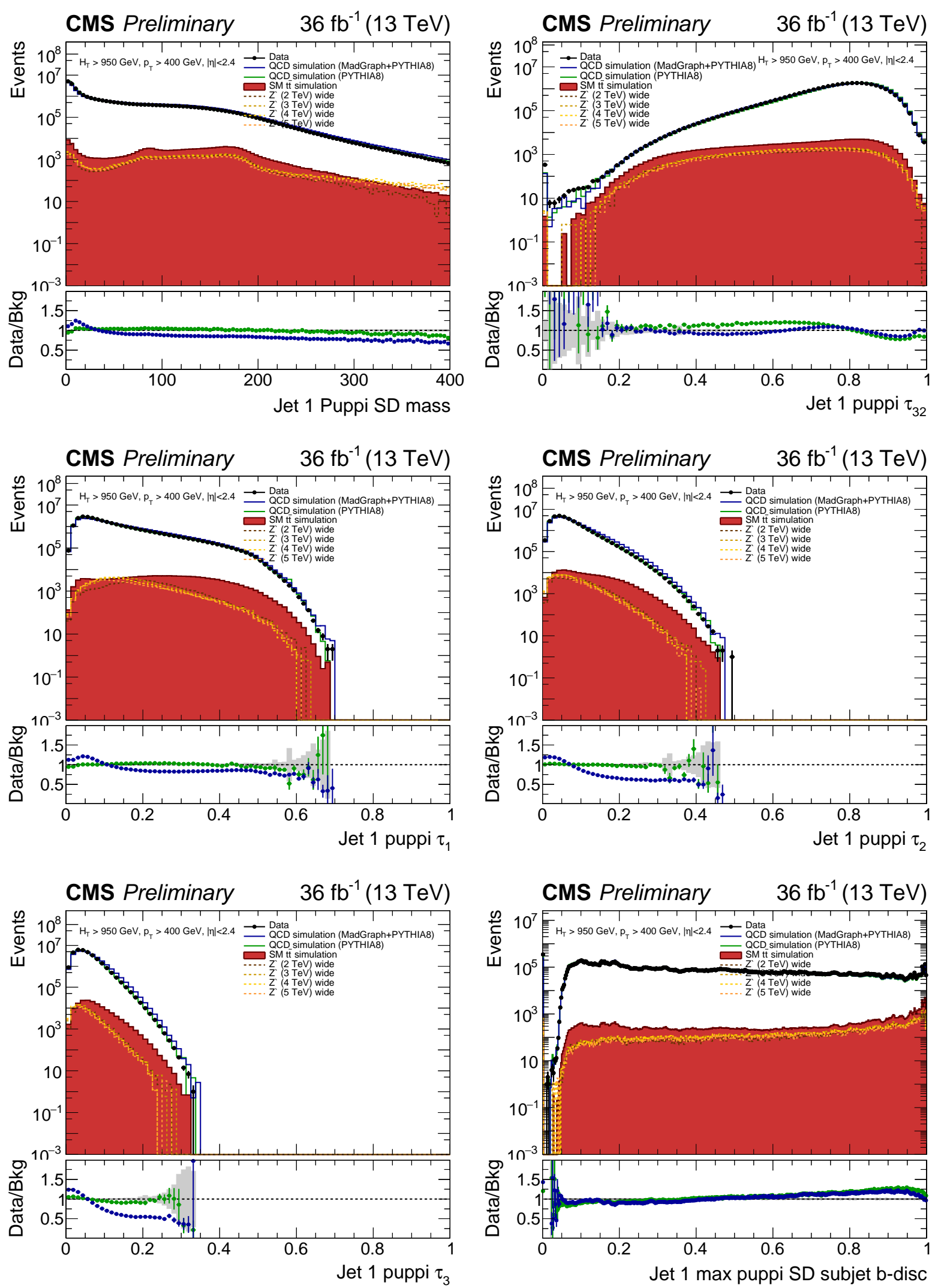

Figure B.0.7: Jet 1 tagging variables after preselection. 

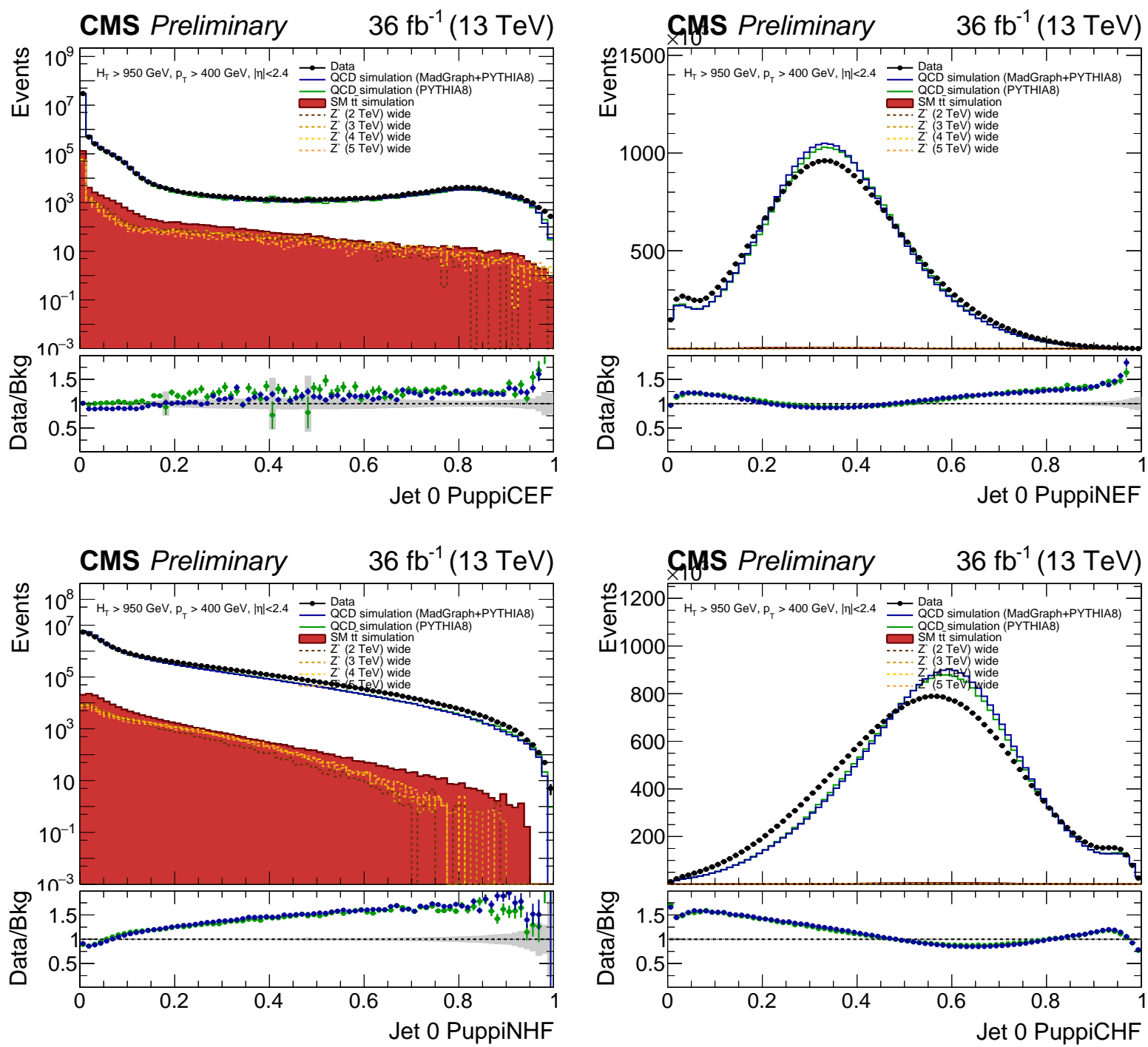

Figure B.0.8: Leading jet PUPPI jet ID variables. 

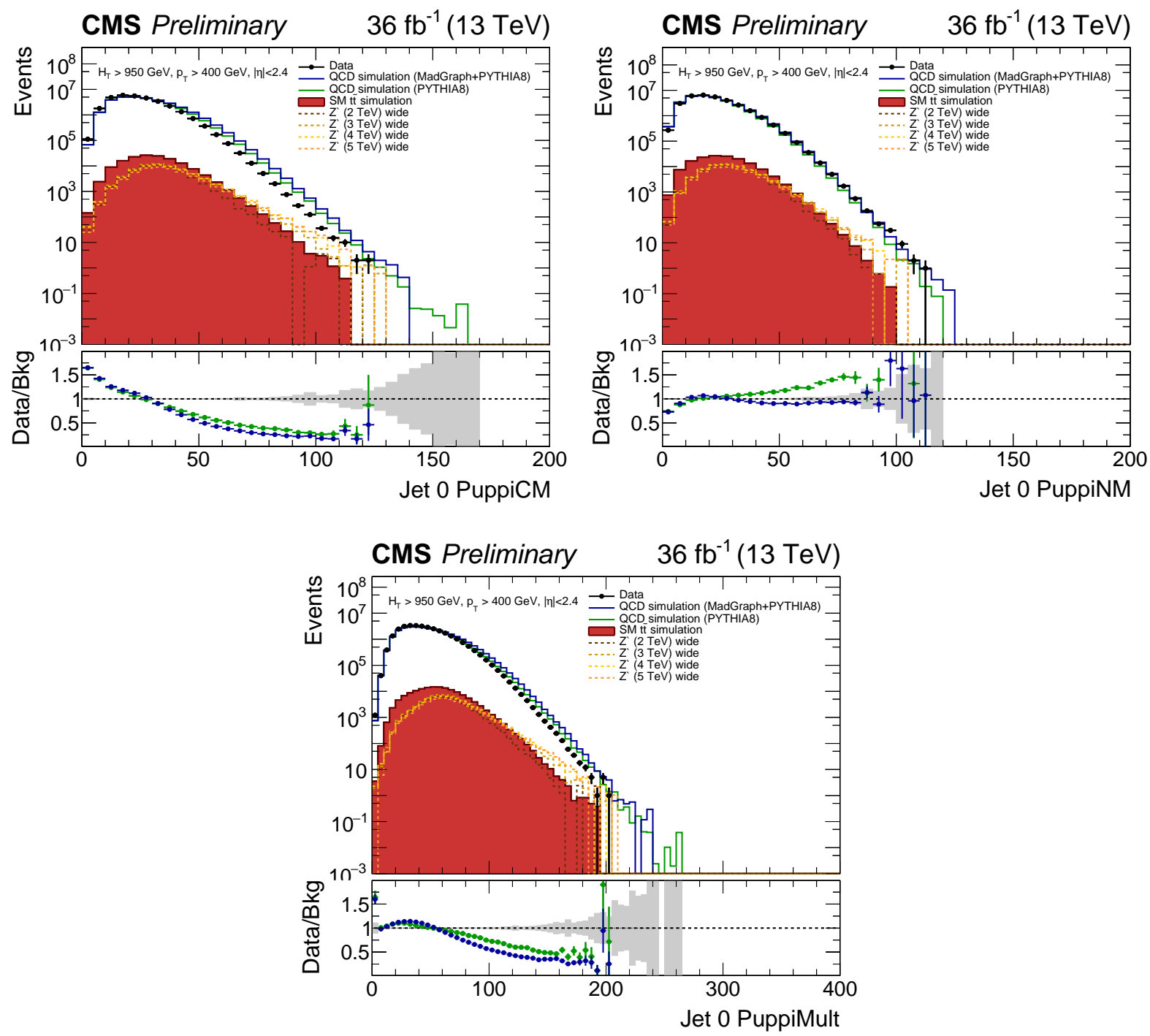

Figure B.0.9: Leading jet PUPPI jet ID variables. 

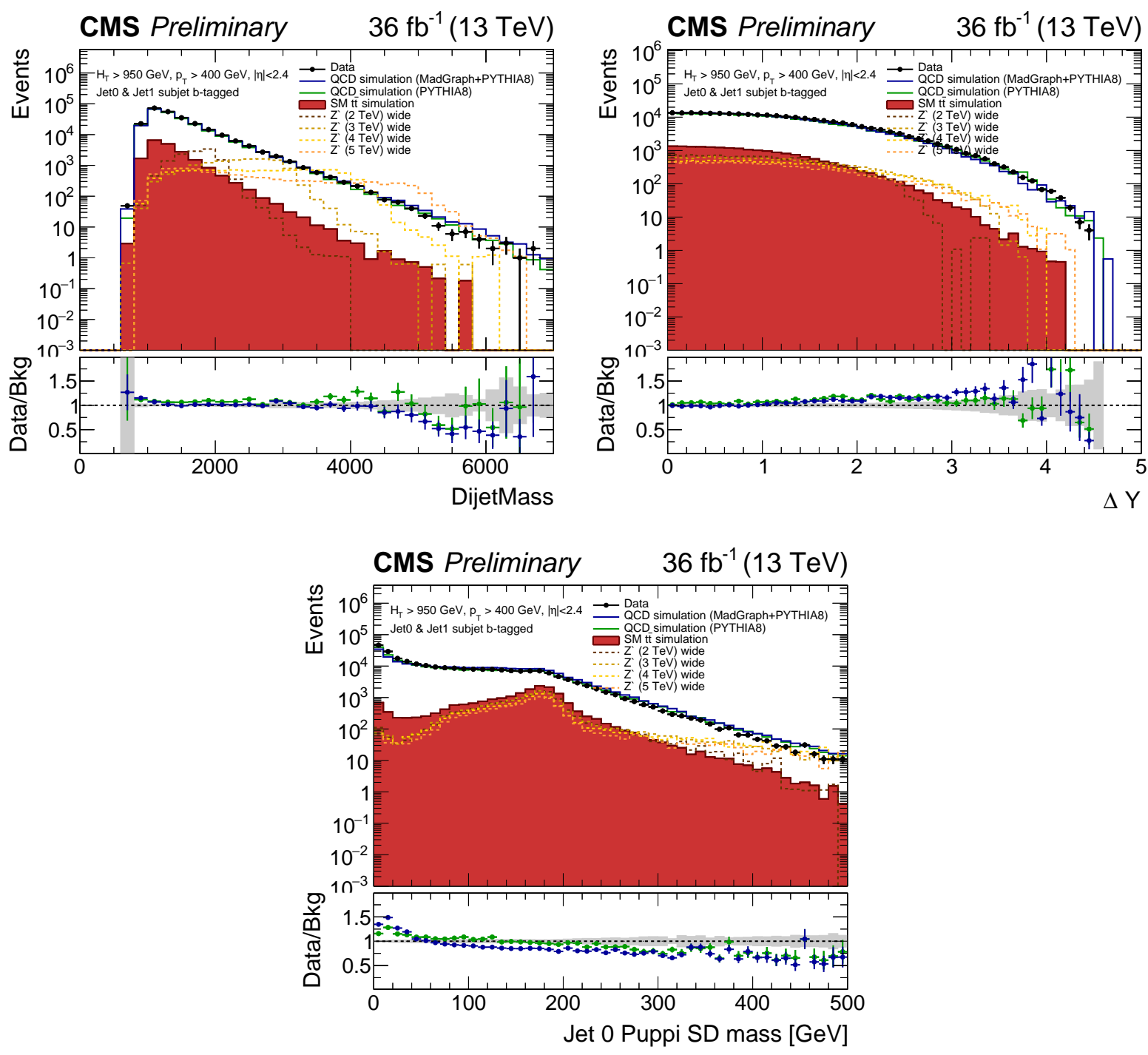

Figure B.0.10: Event kinematic plots after preselection and requiring each jet have a subjet b-tag. 


\section{Appendix C}

\section{Top $p_{T}$ Reweighting}

This section provides plots comparing the effect of top $p_{T}$ reweighting on distributions of the $t \bar{t} \mathrm{MC}$. 

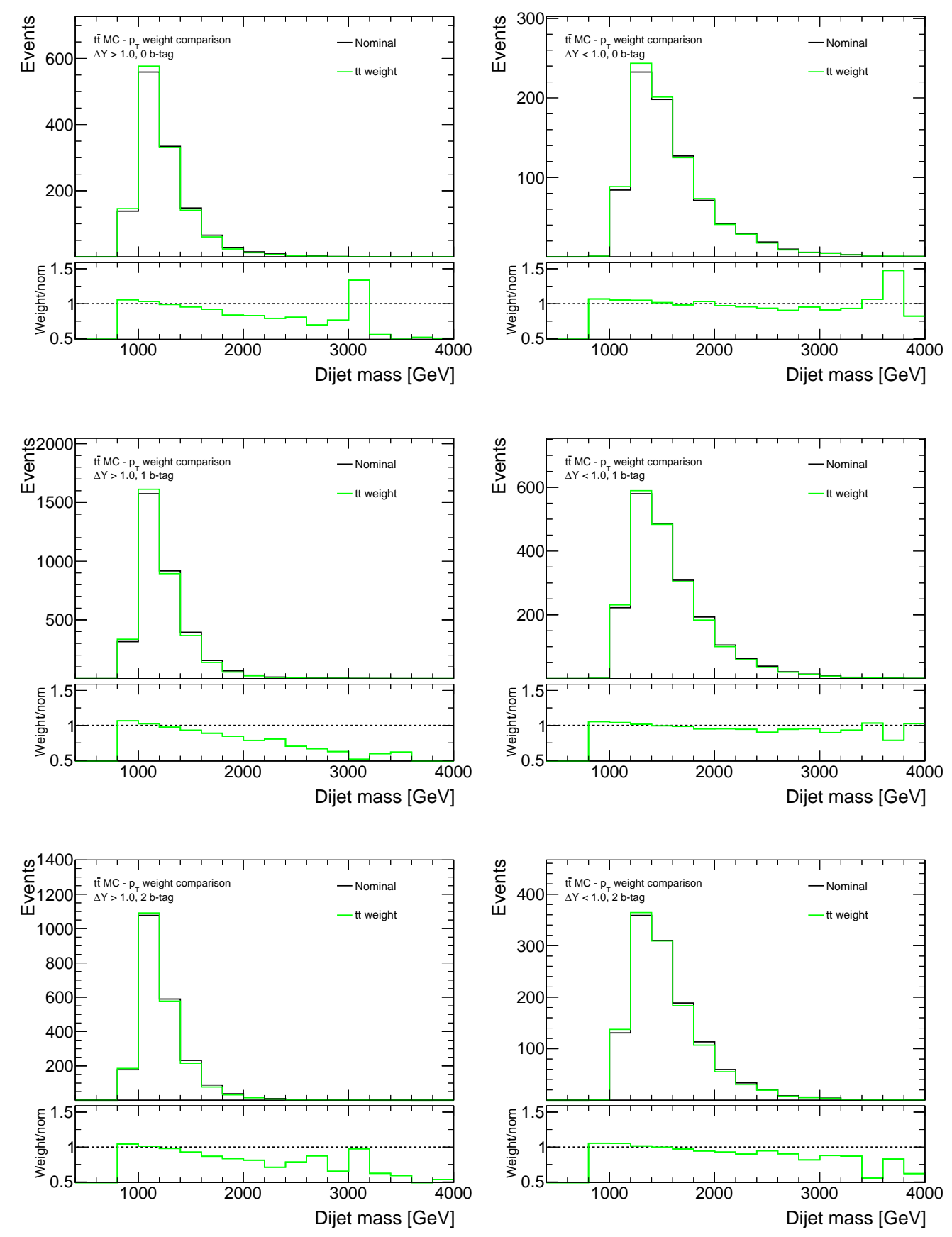

Figure C.0.1: Effect of top $p_{T}$ reweighting on the $t \bar{t}$ Monte Carlo sample for all 6 event categories. 

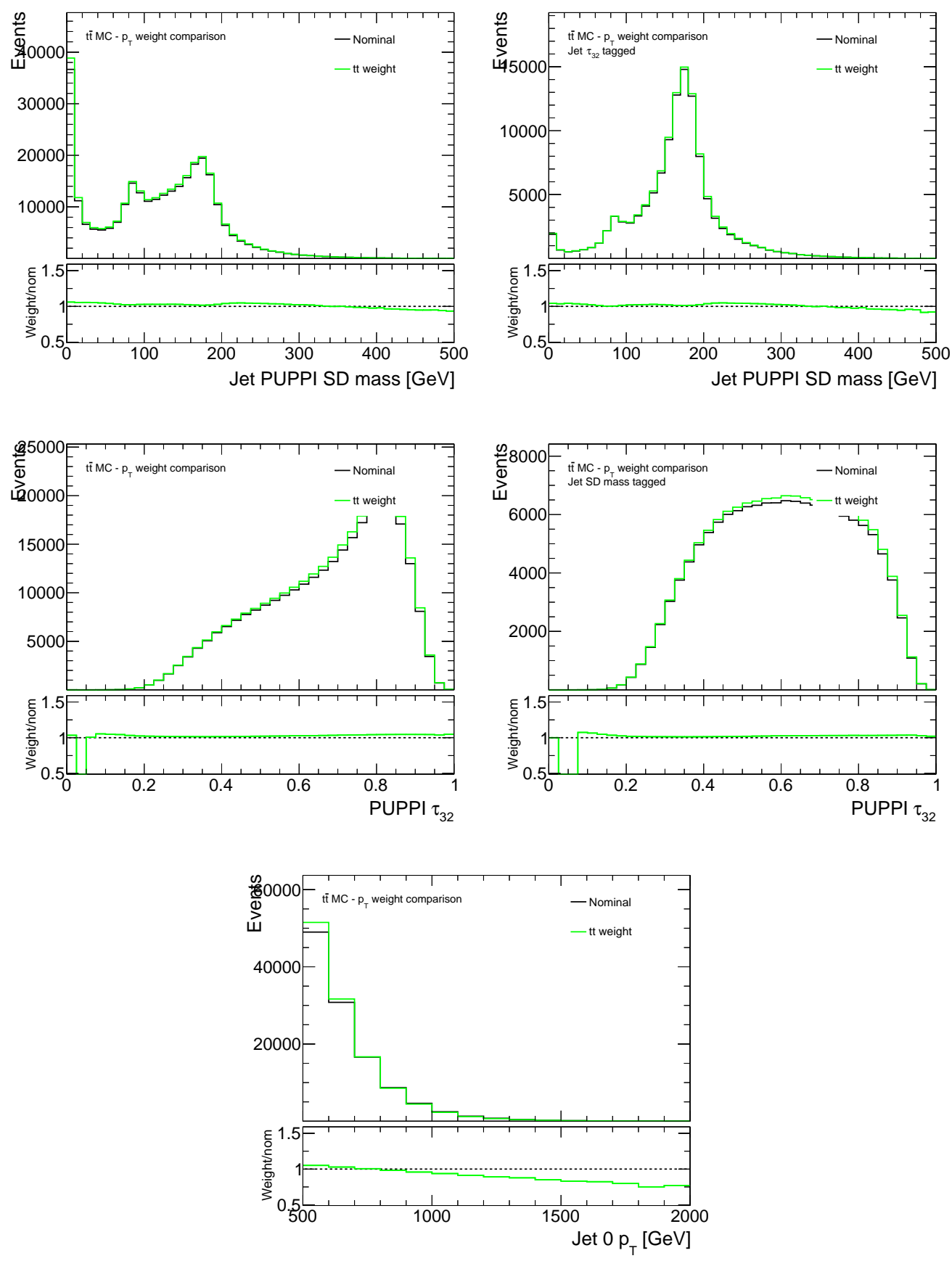

Figure C.0.2: Effect of top $p_{T}$ reweighting on jet variables. 


\section{References}

[1] S. L. Glashow. Partial Symmetries of Weak Interactions. Nucl. Phys., 22:579-588, 1961. doi: 10.1016/0029-5582(61)90469-2.

[2] S. Weinberg. A Model of Leptons. Phys. Rev. Lett., 19:1264-1266, 1967. doi: 10.1103/ PhysRevLett.19.1264.

[3] D. J. Gross and F. Wilczek. Ultraviolet Behavior of Nonabelian Gauge Theories. Phys. Rev. Lett., 30:1343-1346, 1973. doi: 10.1103/PhysRevLett.30.1343.

[4] H. D. Politzer. Reliable Perturbative Results for Strong Interactions? Phys. Rev. Lett., 30:1346-1349, 1973. doi: 10.1103/PhysRevLett.30.1346.

[5] H. Fritzsch, M. Gell-Mann and H. Leutwyler. Advantages of the Color Octet Gluon Picture. Phys. Lett., 47B:365-368, 1973. doi: 10.1016/0370-2693(73)90625-4.

[6] A. Bettini. Introduction to Elementary Particle Physics. Cambridge University Press, 2008.

[7] S. Gasiorowicz. Quantum Physics. John Wiley \& Sons, 3rd edition, 2003.

[8] J. Sakurai and J. Napolitano. Modern Quantum Mechanics. Addison-Wesley, 2nd edition, 2011.

[9] M. E. Peskin and D. V. Schroeder. An Introduction to Quantum Field Theory. Westview Press, 1995.

[10] C. Patrignani et al. Review of Particle Physics. Chin. Phys., C40(10):100001, 2016. doi: 10.1088/1674-1137/40/10/100001.

[11] J. W. Rohlf. Modern Physics from a to Z'. John Wiley \& Sons, 1994.

[12] F. Halzen and A. D. Martin. Quarks \& Leptons: An Introductory Course in Modern Physics. John Wiley \& Sons, 1984.

[13] A. Djouadi. The Anatomy of Electroweak Symmetry Breaking: Tome I: The Higgs Boson in the Standard Model. Physics Reports, 457(1):1 - 216, 2008. ISSN 0370-1573. doi: https://doi.org/10.1016/j.physrep.2007.10.004. 
[14] S. Chatrchyan et al. Observation of a new boson at a mass of $125 \mathrm{GeV}$ with the CMS experiment at the LHC. Physics Letters B, 716(1):30 - 61, 2012. doi: 10.1016/j.physletb. 2012.08.021.

[15] G. Aad et al. Observation of a new particle in the search for the Standard Model Higgs boson with the ATLAS detector at the LHC. Physics Letters B, 716(1):1 - 29, 2012. doi: 10.1016/j.physletb.2012.08.020.

[16] P. Langacker. The Standard Model and Beyond. Taylor \& Francis, 10th edition, 2010.

[17] L. Randall. Extra dimensions and warped geometries. Science, 296(5572):1422-1427, 2002. doi: 10.1126/science.1072567. URL http://science.sciencemag.org/content/ $296 / 5572 / 1422$.

[18] K. Agashe et al. LHC signals from warped extra dimensions. Phys. Rev. D, 77:015003, 2008. doi: 10.1103/PhysRevD.77.015003.

[19] C. T. Hill and S. J. Parke. Top production: Sensitivity to new physics. Phys. Rev., D49:4454-4462, 1994. doi: 10.1103/PhysRevD.49.4454.

[20] J. Rosner. Prominent Decay Modes of a Leptophobic Z'. Phys. Rev. B, 387:113-117, 1996. doi: 10.1016/0370-2693(96)01022-2.

[21] R. Harris, C. Hill and S. Parke. Cross Section for Topcolor Z' decaying to top-antitop. Technical Report FERMILAB-FN-0687, Fermilab, 1999. URL https://arxiv.org/ abs/hep-ph/9911288.

[22] K. Lynch et al. Finding Z' bosons coupled preferentially to the third family at CERN LEP and the Fermilab Tevatron. Phys. Rev. D, 63:035006, 2001. doi: 10.1103/PhysRevD.63.035006.

[23] K. Agashe et al. LHC signals for warped electroweak neutral gauge bosons. Phys. Rev. D, 76:115015, 2007. doi: 10.1103/PhysRevD.80.075007.

[24] L. Evans and P. Bryant. LHC Machine. Journal of Instrumentation, 3(08):S08001, 2008. URL http://stacks .iop.org/1748-0221/3/i=08/a=S08001.

[25] S. Chatrchyan et al. The CMS experiment at the CERN LHC. Journal of Instrumentation, 3(08):S08004, 2008. URL http://stacks.iop.org/1748-0221/3/i=08/a= S08004.

[26] B. Schmidt. The high-luminosity upgrade of the LHC: Physics and technology challenges for the accelerator and the experiments. Journal of Physics: Conference Series, 706(2): 022002, 2016. URL http://stacks . iop.org/1742-6596/706/i=2/a=022002.

[27] L. Rossi. The High Luminosity LHC Project. 2017. URL https://indico. cern.ch/event/647676/contributions/2721132/attachments/1548936/2432720/ 2017-10-30_HL-LHC-Lucio_at_HL-HE_Physics_workshop_v2.pdf. 
[28] V. Khachatryan et al. Technical Proposal for the Phase-II Upgrade of the CMS Detector. Technical Report CERN-LHCC-2015-10, CERN, 2015. URL https://cds.cern.ch/ record/2020886/files/LHCC-P-008.pdf.

[29] G. L. Bayatian et al. CMS Physics: Technical Design Report Volume 1: Detector Performance and Software. Technical report, 2006. URL https://cds.cern.ch/record/ 922757.

[30] V. Karimki et al. The CMS tracker system project: Technical Design Report. Technical report, Geneva, 1997. URL http://cds.cern.ch/record/368412.

[31] A. Colaleo et al. CMS Technical Design Report for the Muon Endcap GEM Upgrade. Technical Report CERN-LHCC-2015-012. CMS-TDR-013, Jun 2015. URL https:// cds.cern.ch/record/2021453.

[32] L. Cadamuro. The CMS Level-1 trigger system for LHC Run II. Journal of Instrumentation, 12(03):C03021, 2017. URL http://stacks .iop.org/1748-0221/12/i=03/ $a=C 03021$.

[33] A. Sirunyan et al. Particle-flow reconstruction and global event description with the CMS detector. Journal of Instrumentation, 12(10):P10003, 2017. URL http://stacks. iop.org $/ 1748-0221 / 12 / i=10 / a=P 10003$.

[34] Y. L. Dokshitzer et al. Better jet clustering algorithms. JHEP, 08:001, 1997. doi: 10.1088/1126-6708/1997/08/001.

[35] S. Catani et al. Longitudinally invariant $K_{t}$ clustering algorithms for hadron hadron collisions. Nucl. Phys., B406:187-224, 1993. doi: 10.1016/0550-3213(93)90166-M.

[36] M. Cacciari, G. P. Salam and G. Soyez. The anti- $k_{t}$ jet clustering algorithm. Journal of High Energy Physics, 2008(04):063, 2008. URL http://stacks.iop.org/1126-6708/ $2008 / i=04 / a=063$.

[37] D. Bertolini et al. Pileup per particle identification. Journal of High Energy Physics, 2014(10):59, Oct 2014. doi: 10.1007/JHEP10(2014)059. URL https://doi.org/10. 1007/JHEP10(2014)059.

[38] D. Krohn, J. Thaler and L.-T. Wang. Jet Trimming. JHEP, 1002:084, 2010. doi: 10.1007/JHEP02(2010)084.

[39] S. D. Ellis, C. K. Vermilion and J. R. Walsh. Recombination Algorithms and Jet Substructure: Pruning as a Tool for Heavy Particle Searches. Phys. Rev., D81:094023, 2010. doi: 10.1103/PhysRevD.81.094023.

[40] J. M. Butterworth et al. Jet substructure as a new Higgs search channel at the LHC. Phys. Rev. Lett., 100:242001, 2008. doi: 10.1103/PhysRevLett.100.242001.

[41] A. J. Larkoski et al. Soft Drop. JHEP, 1405:146, 2014. doi: 10.1007/JHEP05(2014)146. 
[42] J. Thaler and K. Van Tilburg. Identifying Boosted Objects with N-subjettiness. JHEP, 1103:015, 2011. doi: 10.1007/JHEP03(2011)015.

[43] D. E. Kaplan et al. Top Tagging: A Method for Identifying Boosted Hadronically Decaying Top Quarks. Phys. Rev. Lett., 101:142001, 2008. doi: 10.1103/PhysRevLett. 101.142001.

[44] CMS Collaboration. A Cambridge-Aachen (C-A) based jet algorithm for boosted top-jet tagging. CMS Physics Analysis Summary CMS-PAS-JME-09-001, 2009. URL http: //cdsweb.cern.ch/record/1194489.

[45] G. Kasieczka et al. Resonance Searches with an Updated Top Tagger. 2015.

[46] D. E. Soper and M. Spannowsky. Finding physics signals with shower deconstruction. Phys. Rev., D84:074002, 2011. doi: 10.1103/PhysRevD.84.074002.

[47] D. E. Soper and M. Spannowsky. Finding top quarks with shower deconstruction. Phys. Rev., D87:054012, 2013. doi: 10.1103/PhysRevD.87.054012.

[48] Top Tagging with New Approaches. Technical Report CMS-PAS-JME-15-002, CERN, Geneva, 2016. URL https://cds.cern.ch/record/2126325.

[49] CMS Collaboration. Boosted Top Jet Tagging at CMS. CMS Physics Analysis Summary CMS-PAS-JME-13-007, 2014. URL https://cds .cern.ch/record/1647419/.

[50] J. Alwall et al. The automated computation of tree-level and next-to-leading order differential cross sections, and their matching to parton shower simulations. JHEP, 07: 079, 2014. doi: 10.1007/JHEP07(2014)079.

[51] T. Sjstrand et al. An Introduction to PYTHIA 8.2. Comput. Phys. Commun., 191: 159-177, 2015. doi: 10.1016/j.cpc.2015.01.024.

[52] M. Dasgupta et al. Towards an understanding of jet substructure. JHEP, 09:029, 2013. doi: 10.1007/JHEP09(2013)029.

[53] A. M. Sirunyan et al. Identification of heavy-flavour jets with the CMS detector in pp collisions at $13 \mathrm{TeV} .2017$.

[54] T. Plehn, G. P. Salam and M. Spannowsky. Fat Jets for a Light Higgs. Phys. Rev. Lett., 104:111801, 2010. doi: 10.1103/PhysRevLett.104.111801.

[55] T. Plehn et al. Stop Reconstruction with Tagged Tops. JHEP, 1010:078, 2010. doi: 10.1007/JHEP10(2010)078.

[56] A. Hoecker et al. TMVA: Toolkit for Multivariate Data Analysis. PoS, ACAT:040, 2007.

[57] T. Aaltonen et al. Search for resonant production of $t \bar{t}$ pairs in $4.8 \mathrm{fb}^{-1}$ of integrated luminosity of $p \bar{p}$ collisions at $\sqrt{s}=1.96 \mathrm{TeV}$. Phys. Rev. D, 84:072004. doi: 10.1103/ PhysRevD.84.072004. 
[58] V. Abazov et al. Search for $t \bar{t}$ resonances in the lepton+jets final state in $p \bar{p}$ collisions at $\sqrt{s}=1.96$ tev. Phys. Rev. B, 668:98-104, 2008. doi: 10.1016/j.physletb.2008.08.027.

[59] S. Chatrchyan et al. Search for anomalous $t \bar{t}$ production in the highly-boosed allhadronic final state. JHEP, 09:29, 2012. doi: 10.1007/JHEP09(2012)029.

[60] S. Chatrchyan et al. Search for resonant $t \bar{t}$ production in lepton+jets events in $p p$ collisions at $\sqrt{s}=7$ tev. JHEP, 12:15, 2012. doi: 10.1007/JHEP12(2012)015.

[61] G. Aad et al. Search for resonances decaying into top-quark pairs using fully hadronic decays in $p p$ collisions with ATLAS at $\sqrt{s}=7$ tev. JHEP, 01:116, 2013. doi: 10.1007/ JHEP01(2013)116.

[62] G. Aad et al. A search for $t \bar{t}$ resonances in lepton+jets events with highly-boosed top quarks collected in $p p$ collisions with ATLAS at $\sqrt{s}=7$ tev. JHEP, 09:41, 2012. doi: 10.1007/JHEP09(2012)041.

[63] Search for anomalous top quark pair production in the boosted all-hadronic final state using pp collisions at sqrt(s) $=8$ tev. Technical Report CMS-PAS-B2G-12-005, CERN, Geneva, 2013.

[64] S. Chatrchyan et al. Searches for new physics using the $t \bar{t}$ invariant mass distribution in $p p$ collisions at $\sqrt{s}=8$ tev. Phys. Rev. Lett., 111:211804, 2013. doi: 10.1103/ PhysRevLett.111.211804.

[65] V. Khachatryan et al. Search for resonant $t \bar{t}$ production in proton-proton collisions at $\sqrt{s}=8$ tev. Phys. Rev. D, 93:012001, 2016. doi: 10.1103/PhysRevD.93.012001.

[66] G. Aad et al. A search for $t \bar{t}$ resonances using lepton-plus-jets events in proton-proton collisions at $\sqrt{s}=8$ tev with the ATLAS detector. JHEP, 08:148, 2015. doi: 10.1007/ JHEP08(2015)148.

[67] A. M. Sirunyan et al. Search for tt̄ resonances in highly-boosted lepton+jets and fully hadronic final states in proton-proton collisions at $\sqrt{s}=13 \mathrm{TeV} .2017$.

[68] Search for heavy particles decaying to pairs of highly-boosted top quarks using leptonplus-jets events in proton-proton collisions at $\sqrt{s}=13$ tev with the atlas detector. ATLAS Conference Note ATLAS-CONF-2016-014, CERN, 2016.

[69] N. Kidonakis. Differential and total cross sections for top pair and single top production. 2012.

[70] S. Chatrchyan et al. Description and performance of track and primary-vertex reconstruction with the CMS tracker. JINST, 9:P10009, 2014. doi: 10.1088/1748-0221/9/ 10/P10009.

[71] V. Khachatryan et al. Measurement of differential cross sections for top quark pair production using the lepton+jets final state in proton-proton collisions at $13 \mathrm{TeV}$. Phys. Rev., D95(9):092001, 2017. doi: 10.1103/PhysRevD.95.092001. 


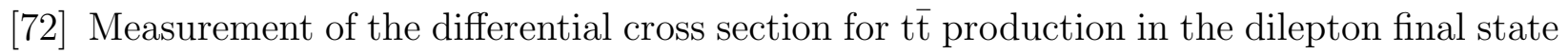
at $\sqrt{s}=13 \mathrm{TeV}$. Technical Report CMS-PAS-TOP-16-011, CERN, Geneva, 2016. URL http://cds.cern.ch/record/2140061.

[73] M. Czakon et al. NNLO QCD predictions for fully-differential top-quark pair production at the Tevatron. JHEP, 05:034, 2016. doi: 10.1007/JHEP05(2016)034.

[74] CMS Collaboration. Jet Energy Corrections and Uncertainties. CMS Detector Performance Plots CMS-DP-2012/012. URL https://cds.cern.ch/record/1460989.

[75] S. Chatrchyan et al. Determination of Jet Energy Calibration and Transverse Momentum Resolution in CMS. JINST, 6:P11002, 2011. doi: 10.1088/1748-0221/6/11/P11002.

[76] CMS Collaboration. Measurement of the differential cross section for top quark pair production in pp collisions at $\operatorname{sqrt}(\mathrm{s})=8 \mathrm{TeV}$. Submitted to EPJC, 2015.

[77] T. Muller, J. Ott and J. Wagner-Kuhr. Theta - a framework for template-based modeling and inference. CMS Internal Note 2010/017, 2010.

[78] R. Bonciani, T. Jezo, M. Klasen, F. Lyonnet, I. Schienbein. Electroweak top-quark pair production at the LHC with Z' bosons to the NLO QCD in POWHEG. JHEP, 02:141, 2016. doi: 10.1007/JHEP02(2016)141.

[79] T. Lenzi. Development of the DAQ System of Triple-GEM Detectors for the CMS Muon Spectrometer Upgrade at LHC. PhD thesis, 2016. URL http://www.iihe.ac. be/publications/thesisLenzi.pdf 